WSRC-TR-2003-00328

Natural and Passive Remediation of Chlorinated Solvents:

Critical Evaluation of Science and Technology Targets

February 19, 2004

Prepared by a National Technical Working Group

Westinghouse Savannah River Company

Savannah River Site

Aiken, SC 29808

Prepared for the United States Department of Energy under Contract No. DE-AC09-96-SR18500 
This document was prepared in conjunction with work accomplished under Contract No. DE-AC09-96SR18500 with the U. S. Department of Energy.

\section{DISCLAIMER}

This report was prepared as an account of work sponsored by an agency of the United States Government. Neither the United States Government nor any agency thereof, nor any of their employees, makes any warranty, express or implied, or assumes any legal liability or responsibility for the accuracy, completeness, or usefulness of any information, apparatus, product or process disclosed, or represents that its use would not infringe privately owned rights. Reference herein to any specific commercial product, process or service by trade name, trademark, manufacturer, or otherwise does not necessarily constitute or imply its endorsement, recommendation, or favoring by the United States Government or any agency thereof. The views and opinions of authors expressed herein do not necessarily state or reflect those of the United States Government or any agency thereof.

This report has been reproduced directly from the best available copy.

Available for sale to the public, in paper, from: U.S. Department of Commerce, National Technical Information Service, 5285 Port Royal Road, Springfield, VA 22161, phone: (800) 553-6847, fax: (703) 605-6900

email: orders@ntis.fedworld.gov

online ordering: http://www.ntis.gov/help/index.asp

Available electronically at http://www.osti.gov/bridge

Available for a processing fee to U.S. Department of Energy and its contractors, in paper, from: U.S. Department of Energy, Office of Scientific and Technical Information, P.O. Box 62, Oak Ridge, TN 37831-0062,

phone: (865)576-8401,

fax: (865)576-5728

email: $\underline{\text { reports@ adonis.osti.gov }}$ 


\title{
Natural and Passive Remediation of Chlorinated Solvents: Critical Evaluation of Science and Technology Targets
}

\author{
U.S. Department of Energy Office of Science and Technology \\ U.S. Department of Energy Savannah River Operations \\ Westinghouse Savannah River Company
}

\author{
Technical Working Group \\ Frank Chapelle, USGS \\ Tom Early, ORNL \\ Tyler Gilmore, PNNL \\ Michael Heitkamp, SRTC \\ Brian Looney, SRTC \\ David Major, GeoSyntec, Inc. \\ Gary Wein, BSRI \\ Todd Wiedemeier, T. H. Wiedemeier \& Associates \\ Ex Officio Members \\ Karen Vangelas, Savannah River Operations Team Lead, SRTC \\ Karen Adams, Savannah River Operations Office Representative, US DOE \\ Ken Lovelace, Office of Emergency and Remedial Response, US EPA \\ Claire Sink, Office of Cleanup Technologies, Project Manager, US DOE
}


Acknowledgements

The MNA/EPR Technical Working Group would like to acknowledge those who played a key role in the development of this work. The United States Department of Energy (US DOE) Office of Cleanup Technologies sponsored this effort. We appreciate the guidance and support of Claire Sink of US DOE Headquarters and Tom Treger and Karen Adams of US DOE Savannah River. We thank all the project participants and contributing authors for their time and thoughtful contributions. We acknowledge the participation and collaboration of other federal agencies, notably, the U.S. Geological Survey (USGS) and the U.S. Environmental Protection Agency (US EPA).

We would like to extend our specific acknowledgement to the following people and organizations.

- The project management and operations team:

Claire Sink US DOE, EM-21

Tom Treger, $\quad$ US DOE, Savannah River Operations

Karen Adams $\quad$ US DOE, Savannah River Operations

Karen Vangelas Savannah River Technology Center (SRTC)

Brian Looney SRTC

Bob Aylward SRTC

Jack Corey SRTC

Mike Shotten Bechtel Savannah River, Inc., Soils and Groundwater Closure Project

Tyler Gilmore $\quad$ Pacific Northwest National Laboratory (PNNL)

Tom Early Oak Ridge National Laboratory (ORNL)

- The lead authors for the lines of inquiry and historical/regulatory overviews:

Charles J. Newell Groundwater Services, Inc.

Ken Lovelace US EPA, Office of Emergency and Remedial Response

Frank Chapelle USGS

Tom Early ORNL

Gary Wein BSRI

Dave Major GeoSyntec, Inc.

Michael Heitkamp SRTC

Tyler Gilmore $\quad$ PNNL

Jody Waugh S. M. Stoller

Todd Wiedemeier T. H. Wiedemeier \& Assoc., LLC

Brian B. Looney SRTC

- Ken Lovelace of the US EPA Office of Emergency and Remedial Response for providing the team with a national regulatory perspective on monitored natural attenuation of chlorinated solvents.

- Norm Cutshall, EG\&G for his expert support to the project management and operations team. 
- Jeanne Malanowski, SRTC; Jennifer Law and Troy Regis, US DOE HQ interns, for their skilled assistance in preparing this document.

The lead authors and contributing authors for each line of inquiry are provided below. We thank all authors and contributing authors for their contributions.

Natural Processes - No Enhancements. Lead Author: Frank Chapelle, USGS. Contributing Authors: Celeste Journey, USGS; Dave Major, Kevin Finneran, GeoSyntec, Inc.; Tom Early, ORNL; Todd Wiedemeier, T.H. Wiedemeier \& Assoc., LLC; Tyler Gilmore, Robert G. Riley, PNNL; Gary Wein, BSRI; Brian Looney, Robin Brigmon and Michael Heitkamp, SRTC.

Natural Processes - Enhancements. Lead Author: Tom Early, ORNL. Contributing Authors: T. J. Tscaplinski, ORNL; Dave Major, GeoSyntec; Michael Heitkamp, Robin Brigmon, SRTC; Gary Wein, BSRI; Todd Wiedemeier, T. H. Wiedemeier \& Assoc., LLC; and Jody Waugh, S. M. Stoller.

Interfaces. Lead Author: Gary Wein, BSRI. Contributing Author: Brian Looney, SRTC.

Scenarios. Lead Author: Brian Looney. Contributing Author: Charles J. Newell, Groundwater Services, Inc.

Strategy for Characterization and Longterm Monitoring. Lead Author: Tyler Gilmore, PNNL. Contributing Authors: Brian Looney, Brian Riha, SRTC; Jody Waugh, S. M. Stoller.

Multiple Lines of Evidence. Lead Author: Todd Wiedemeier, T. H. Wiedemeier \& Assoc., LLC. Contributing Authors: Dave Major, GeoSyntec, Inc.

Non-Standard Monitoring Concepts. Lead Author: Brian Looney, SRTC. Contributing Author: Tom Early, W. E. Doll, and L. P. Beard, ORNL.

Modeling. Lead Author: Brian Looney, SRTC. Contributing Author: Mike Truex, PNNL.

Sensors. Lead Author: Brian Looney, SRTC. Contributing Author: Joe Rossabi, SRTC.

Configuration of Monitoring Systems. Lead Author: Jody Waugh, S. M. Stoller. Contributing Authors: Brian Riha, Brian Looney, SRTC; and Tyler Gilmore, PNNL.

Advanced Bioassessment Tools. Lead Author: Dave Major, GeoSyntec, Inc. Contributing Authors: Michael Heitkamp and Christopher Bagwell, SRTC.

Regulatory Perspective. Lead Author: Ken Lovelace, US EPA. Contributing Author: Brian Looney, SRTC. 
Historical Perspective. Lead Author: Charles J. Newell, Groundwater Services, Inc. Contributing Authors: Travis McGuire, Carol E. Aziz, Tim Nickels, Groundwater Services, Inc.; Brian Looney and Karen Vangelas, SRTC. 
WSRC-TR-2003-00328

February 19, 2004

\{blank page 


\section{Table of Contents}

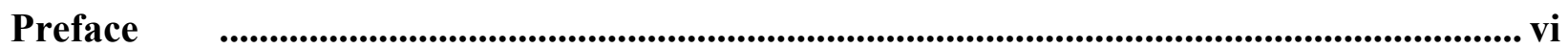

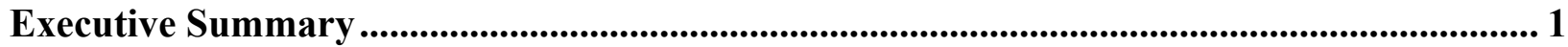

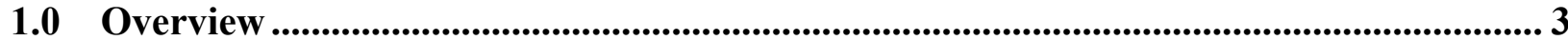

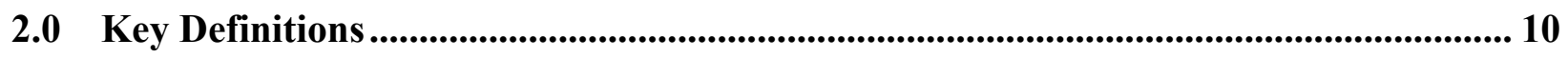

3.0 Historical and Regulatory Perspective........................................................................ 11

3.1 Historical Background ....................................................................................................... 11

3.1.1 The Survey ........................................................................................................... 12

3.1.2 The Survey Results ..................................................................................... 13

3.1.3 Key Conclusions from the Survey ................................................................. 13

3.2 Regulatory and Peer Review Perspective................................................................ 14

4.0 Critical Evaluation Process ............................................................................................. 36

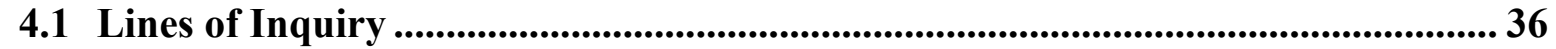

4.1.1 Scientific Basis for MNA/EPR - Process Lines of Inquiry ........................ 37

4.1.2 Characterization and Monitoring Lines of Inquiry................................... 39

4.2 Output from the Line of Inquiry Evaluations ..................................................... 41

5.0 Scientific Basis for MNA and EPR................................................................... 42

5.1 Summary of Natural Attenuation Processes--Without Enhancements................. 42

5.2 Enhanced Passive Remediation Opportunities.................................................... 45

5.2.1 Microbiology ................................................................................................................ 48

5.2.2 Abiotic Processes....................................................................................... 50

5.2.3 Phytoremediation .................................................................................................... 51

5.2.4 Active Processes at System Interfaces - Potential Role in MNA/EPR..... 54

5.2.5 Summary for Large Scale Hydraulic Modifications .................................56

5.2.6 Scenario Tools for Evaluating MNA ...................................................... 58

6.0 Documenting and Monitoring MNA and EPR.............................................................60 60

6.1 Overall Characterization and Monitoring Strategy for MNA and EPR ............. 60

6.1.1 Background ..............................................................................................................6 60

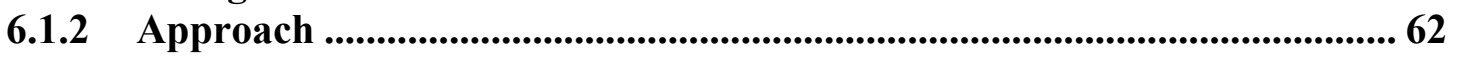

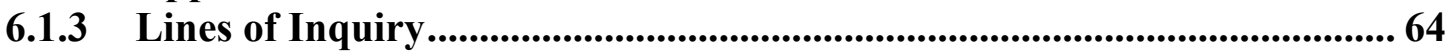

6.1.4 Characterization ......................................................................................................6 66

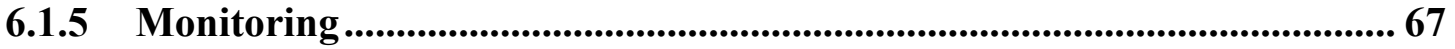

6.2 Multiple Lines of Evidence ..............................................................................6 67

6.3 Characterization and Monitoring Opportunities .................................................... 69

6.3.1 Advanced Bioassessment Tools.................................................................... 69

6.3.2 Non-Standard Monitoring Strategies...................................................... 72

6.3.3 Fate and Transport Modeling - Directions and Opportunities to Support and Accelerate Natural Attenuation and Remediation .............................. 73

6.3.4 Sensors ............................................................................................................. 77

6.3.5 Monitoring System Configuration .................................................................... 81

7.0 Identification of Short Term and Long Term Science and Technology Targets ......... 82

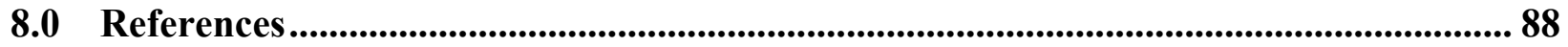

9.0 Appendices Large Prioritization Tables .................................................................. 123 


\section{List of Tables}

Table 1. Considerations for Selecting Modeling Approach Based on Site Properties ... 75 Table 2. High Priority Technical and Implementation Targets Recommended for US DOE MNA/EPR Alternative Project ..................................................................... 84

Table 3. High Priority Policy Targets Recommended for US DOE MNA/EPR Alternative Project...................................................................................................... 86

Table 4. High Priority Technical Targets for consideration by basic science research programs such as EMSP and SERDP ............................................................... 87 


\section{List of Figures}

Figure 1 Continuum of Individual Remediation Technologies........................................5

Figure 2 Schematic diagram of contaminant loading and natural attenuation capacity in ground-water systems.

Figure 3 Recent Historical Development of MNA/EPR for Petroleum Hydrocarbons and Chlorinated Solvents (modified from Wiedemeier and Barden, 2002) ..... 12

Figure 4 Graphs of Historical Survey Results (follow) ................................................. 16

Figure 5 Lines of inquiry for the principle components of natural attenuation where enhancements may improve their efficiency. .................................................. 47

Figure 6 Locations within a watershed most favorable for introducing enhancements to natural processes.

Figure 7 Simplified schematic diagram of the major zones potentially traversed by a chlorinated solvent plume. I = vadose zone, II = groundwater, III = "wetland",

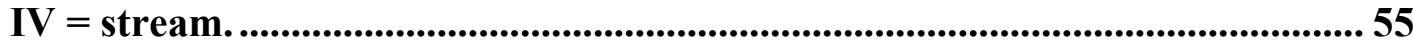

Figure 8 Mass balance between contaminant loading and system capacity .................... 61

Figure 9 Characterization/Monitoring Transition.................................................................. 63

Figure 10 Relative importance of each Line of Inquiry at each phase of the characterization and monitoring process. ..............................................................66 66

Figure 11 Application of Biomolecular Tools for MNA ...................................................... 71 


\section{Acronyms}

\begin{tabular}{|c|c|}
\hline $\mathrm{ACL}$ & alternate concentration limit \\
\hline APHIS & Animal and Plant Health Inspection Service \\
\hline ASTM & American Society for Testing and Materials \\
\hline $\mathrm{CAB}$ & Citizens Advisory Board \\
\hline CERCLA & Comprehensive Environmental Response, Compensation and Liability Act \\
\hline CMP & Chemical, Metals and Pesticides \\
\hline $\mathrm{CMS} / \mathrm{FS}$ & Corrective Measures Study/Feasibility Study \\
\hline Cs-137 & cesium 137 \\
\hline CVOC & Chlorinated volatile organic contaminants \\
\hline DCA & di-Chloroethane \\
\hline cis-DCE & cis-Di-Chloroethylene \\
\hline trans-1,2-DCE & trans-1,2- di-Chloroethylene \\
\hline DNA & deoxyribonucleic acid \\
\hline DNAPL & Dense Non-Aqueous Phase Liquids \\
\hline $\mathrm{DO}$ & dissolved oxygen \\
\hline EMSP & Environmental Management Science Program \\
\hline EPR & Enhanced Passive Remediation \\
\hline FRAP & Field Research Advisory Panel \\
\hline FS & Feasibility Study \\
\hline FUSRAP & Formerly Utilized Sites Remedial Action Program \\
\hline GW & groundwater \\
\hline HQ & headquarters \\
\hline ITRC & InterState Technology Regulatory Council \\
\hline IWG & Interactive Working Group \\
\hline LIDAR & Light Detection and Ranging \\
\hline LNAPL & Light Non-Aqueous Phase Liquids \\
\hline MCL & maximum contaminant limit \\
\hline MNA/EPR & monitored natural attenuation/enhanced passive remediation \\
\hline $\mathrm{N}$ & Nitrogen \\
\hline NABIR & Natural and Accelerated Bioremediation Research \\
\hline NAC & Natural Attenuation Capacity \\
\hline NAPL & Non-Aqueous Phase Liquid \\
\hline NAS & National Academy of Science \\
\hline $\mathrm{NRC}$ & National Research Council \\
\hline ORNL & Oak Ridge National Laboratory \\
\hline OSWER & Office of Solid Waste and Emergency Response \\
\hline OU & Operable Unit \\
\hline P\&T & pump and treat \\
\hline PCE & tetrachloroethylene \\
\hline PI & principal investigators \\
\hline PNNL & Pacific Northwest National Laboratory \\
\hline PRB & Permeable Reactive Barrier \\
\hline
\end{tabular}


RAO

RCRA

ROD

RTDF

RT3D

SC DHEC

SERDP

SGCP

SR

SREL

SRS

SROT

SRTC

SSEB

STAR

SVE

SW

S\&T

S\&T Report

TCA

TCE

TOC

TWG

UMTRA

US DOD

US DOE

US EPA

USGS

$\mathrm{VC}$

VOC

$\mathrm{VZ}$

WSRC
Remedial Action Objective

Resource Conservation and Recovery Act

Record of Decision

Remediation Technologies Development Forum

Reactive Transport in 3-Dimensions

South Carolina Department of Health and Environmental Control

Strategic Environmental Research and Development Program

Soils and Groundwater Closures Project

Savannah River

Savannah River Ecology Laboratory

Savannah River Site

Savannah River Operations Team

Savannah River Technology Center

Southern States Energy Board

Science to Achieve Results

Soil Vapor Extraction

surface water

Science and Technology

"Natural and Passive Remediation of Chlorinated Solvents: Critical

Evaluation of Science and Technology Targets" document

trichloroethane

trichloroethylene

total organic carbon

Technical Working Group

Uranium Mill Tailing Remedial Action Program

United States Department of Defense

United States Department of Energy

United State Environmental Protection Agency

United States Geological Survey

vinyl chloride

volatile organic compounds

vadose zone

Westinghouse Savannah River Company 


\section{Preface}

This report was written for multiple audiences. Each reader will likely approach the subject from a different perspective, seeking different information. The team that developed the document consisted of several technical experts on MNA and included participants who were integral in the development of US EPA's chlorinated solvent MNA protocol (US EPA, 1998) and the subsequent US EPA/OSWER policy directive (OSWER Directive 9700.4-17P; US EPA, 1999). The primary assignment to our interdisciplinary team was to generate a summary of the state-ofthe-art, particularly for chlorinated solvent applications and to identify high-priority opportunities for advancement. A diverse jury of peers reviewed the draft report; we edited the report in response to their comments. In fact, the preface is included as a response to a peer reviewer recommendation.

Potential technology users of MNA should find the report helpful in their decisions on site remediation planning. The rapid increase in selections of MNA as the sole remedy clearly indicates user interest in the approach. As a succinct documentation of the capabilities and limitations of MNA, the report provides a good introduction. The extensive list of References provides a complete reference library.

Regulators may use the document for information on possible forthcoming technological advances in MNA. We consulted numerous regulators during preparation of the report to identify current issues or concerns from their perspective. We anticipate the results of the technical projects to be the basis for addressing the most prominent regulatory issues in an update to the current protocol for implementing MNA. To this end, we formed a partnership with the Interstate Technology Regulatory Council to integrate the new results into technical regulatory guidance. This partnership is described in detail in the project implementation plan (WSRC, 2003).

Science and technology developers may scan the document for innovative ideas or for matching their research interests and capabilities with the areas deemed high-priority. We expect a suite of short-term research projects will be funded to pursue those areas. Project selection is scheduled for January 2004 and completion is planned by December 2005.

Stakeholders and general readers should find the document useful as a primer on MNA and the more aggressive Enhanced Passive Remediation. The section on historical review and the supporting document that fully reports the results of that review should be of particular interest as they provide important insight into hands-on experience.

As the project management team, we intend to use the report as a guide for expediting the use of MNA for chlorinated ethenes at Savannah River Site and elsewhere at US DOE sites. We intend for the results of the project to be used initially in Records of Decision due in May 2005 and February 2007. 


\section{Executive Summary}

Efficient, effective and responsible use of Monitored Natural Attenuation (MNA) and Enhanced Passive Remediation (EPR) for chlorinated solvents is critical to the US Department of Energy (US DOE) as the organization shifts its efforts to site closure. Chlorinated solvents represent many of the largest and most challenging plumes at US DOE sites across the country - including the Savannah River Site, Oak Ridge Site, and the Hanford Site. To facilitate implementation of MNA and EPR, the US DOE Office of Environmental Management has sponsored an Alternative Project. The project is narrowly focused to provide the scientific and policy support to facilitate implementing appropriate passive cleanup and cost effective monitoring strategies leading to responsible completion of active remediation activities at high risk US DOE waste sites. To meet this goal efficiently, a technical working group with broad national representation was formed to strategically guide the project. The initial result of their efforts is a list of specific-integrated research and development targets that will serve to focus and guide the Monitored Natural Attenuation and Enhanced Passive Remediation for Chlorinated Solvents US DOE Alternative Project for Technology Acceleration (hereafter referred to as the US DOE MNA/EPR Alternative Project).

- The evaluation process consisted of several steps. The team identified several lines of inquiry - general research and development areas that show promise in facilitating MNA/EPR. Each of these lines of inquiry was assigned to a subgroup for detailed and critical evaluation. The line of inquiry evaluation in turn was used to identify a few items that show particular promise to facilitate the implementation of MNA/EPR. The overall list was prioritized into an integrated portfolio as described below.

- The entire sequence proved to be quite effective. The initial evaluation in the lines of inquiry reduced the potential list of research topics from an essentially unlimited set to a short list of 47 items to prioritize. In the end, the process identified 18 high priority technical targets for this project. The various high priority technical items were supportive and integrated. The team also identified 4 high priority policy targets and a significant number of basic science (long-term research) targets.

- MNA/EPR relies on benefical use of natural processes such as degradation, immobilization and dispersion to reach environmental goals. In other words, the attenuation capacity of the system must be greater than or equal to the contaminant input, or loading.

- In keeping with the central technical concept, the team emphasized four major factors in the prioritization evaluation: 1) the item should support the framework of attenuation capacity and loading, 2) the item should support the transition of a site into a protective cost effective system performance monitoring state, 3 ) the item should move toward direct and integrated measures of processes (rather than indirect measures), and 4) enhancements for EPR should be naturally sustainable. Direct measures were deemed to be most useful to support characterization and early monitoring stages while integrating/surrogate measures were 
deemed to be useful for long-term system performance monitoring. The team also considered the timeline and cost for the development to ensure alignment with the US DOE MNA/EPR Alternative Project constraints. By emphasizing this short list of criteria, the team felt the ultimate portfolio would consist of synergistic items that will move MNA/EPR strongly in the desired direction.

- The highest rated technical targets were the advanced bioassessment tools. These tools include molecular probes and microarrays that provide a more direct measure of attenuation capacity. Some of the probes identify the specific degraders and their degradation activity. This is a powerful tool to assess MNA/EPR and could streamline documentation. These tools are rapidly increasing in capability and are falling in cost due to high volume production and use for medical diagnostics, genomics and proteomics.

- Other characterization and monitoring priorities included: development of a scenario-based key to support matching monitoring and modeling to site specific conditions, new types of sentinel monitoring systems, measurement of "irreversible" sorption, direct flux measurements, and a range of specific sensors.

- There were three modeling priorities that addressed specific key developments that would support integration of modeling into MNA/EPR and to align the modeling with the attenuation capacity-loading paradigm.

- There were two promising process enhancement (EPR) concepts including bioaugmentation and large-scale hydrologic modification. Phytoremediation was positively reviewed by the team but was assigned a medium priority primarily because much work is already funded in this area and relevant project specific studies would require an extended period of time. 


\subsection{Overview}

Monitored Natural Attenuation (MNA) and Enhanced Passive Remediation (EPR) are straightforward strategies that are based on the natural and sustainable processes that degrade and attenuate contaminants at every site. To put this concept into beneficial use, there are two requirements. First, the principal degradation and attenuation processes at the site must be verified and their attenuation capacity estimated. Second, the resulting information needs to be evaluated to determine if the attenuation capacity within an acceptable distance is "enough" to attain site-specific environmental and regulatory objectives. This conceptual approach advocates working throughout the plume to quantify attenuation capacity. If the capacity of the system is not enough to address the contaminant plume, it could be determined what incremental increase in capacity would be required to adequately address the plume. Such a combined calculation approach provides a powerful conceptual basis for improved environmental management and decision-making.

Chlorinated solvents are subject to a variety of physical, chemical, or biological processes that lead to natural attenuation. While degradation processes are the most desirable, all of these mechanisms can play a useful role in MNA and EPR. The strategy of balancing attenuation capacity with potential for contaminant loading from the source leads directly to technically based approaches for encouraging the beneficial use of natural processes and for improving documentation of performance while minimizing costs and mitigating any adverse collateral impacts. In particular, such a strategy suggests the following three objectives:

- Promote activities that expand the options for natural and sustainable remediation, and to ensure that attenuation capacity is accurately estimated,

- Promote the application of passive methods to reduce contaminant loading and enhance the balance of loading and attenuation, and

- Integrate measures of conditions or parameters that relate to either attenuation capacity or contaminant delivery that may provide improved monitoring and expanded confidence in system performance.

The first two items provide a qualitative description of the opportunities for EPR versus MNA. The last item provides a description of monitoring that may improve implementation and assure that risks from the site are mitigated for both EPR and MNA.

This "attenuation capacity" based paradigm has the potential to build on traditional measures of plume stability and geochemical footprints (US EPA, 1998; NRC, 2001). The ideas support recommendations from the US EPA that stress that natural attenuation is most appropriate when used in conjunction with engineered reduction of contaminant sources or as a follow up measure. The precise manner in which active remediation and MNA/EPR are combined depends on the natural attenuation capacity (NAC) of the system. If the NAC is relatively small, then active measures will be needed to remove or immobilize a significant proportion of the contaminant source. If the NAC is relatively large (or if it can be enhanced appropriately), less active source 
treatment may be necessary for MNA to be successfully employed. In either case, it is necessary to verify and quantify the attenuation capacity to effectively implement this approach.

Environmental remediation technologies can be viewed on a continuum ranging from highly invasive source excavation on one end to non-invasive MNA on the other end (Figure 1). This continuum represents technologies and strategies used during the period of remediation. Over time these approaches reduce and attenuate risks to meet the remediation goals. This results in a final status where the site requires no further action. As drawn, aggressive technologies such as direct in situ chemical destruction and thermal methods fall near the left of the continuum. Baseline pump and treat, active bioremediation and similar methods are near the center of the continuum. Permeable treatment systems fall to the right. Importantly, the area adjacent to MNA on the continuum is designated EPR. This includes technologies that can be implemented and performance will be sustained in a manner analogous to MNA (e.g., permanently modifying hydrology or the special case of bioaugmentation with a missing microorganism). The two requirements for MNA/EPR, as described in the first paragraph, are the key to defining the allowable boundary of EPR. For sites where contaminant delivery from the source is less than the naturally sustainable attenuation capacity (after enhancements, if appropriate), then the site falls to the right of the dashed line and EPR is viable. Conversely, for sites where contaminant delivery from the source is greater than the naturally sustainable attenuation capacity, then the site falls to the left of the dashed line and active remediation will be necessary. This continuum eliminates the historical dichotomy that has been strictly drawn between active remediation and natural attenuation. When combined with attenuation capacity, the continuum provides a quantitative basis for determining when MNA and EPR are useful and appropriate and what activities logically fit into the classification. Importantly, a majority of contaminated sites will require a sequence of activities for responsible cleanup - often including source removal/destruction combined with treatment of a primary contaminant plume (the soil and groundwater that contain moderate to high concentrations). Virtually all sites will have a monitored natural attenuation component, either in the more dilute portion of the plume or after transitioning from more active treatment actions. The capacity based definitions and continuum will support a technical determination of how and when to transition from active remediation to MNA/EPR.

\section{The Challenge -- Sources of Complexity in MNA and EPR}

MNA and EPR are approaches that are based on the deliberate use of natural contaminant degradation/dispersion/immobilization processes to reach site-specific remediation goals. In principle, the effectiveness of MNA/EPR can be evaluated as a simple balance between contaminant delivery to, and contaminant removal from, ground-water systems. Experience has shown that technically reliable evaluations of MNA/EPR are much more complex than is suggested by the simple concept of mass balance. Understanding the sources of this complexity, and understanding why this complexity is inevitable, is fundamental to making technical assessments of MNA/EPR in ground-water systems. 


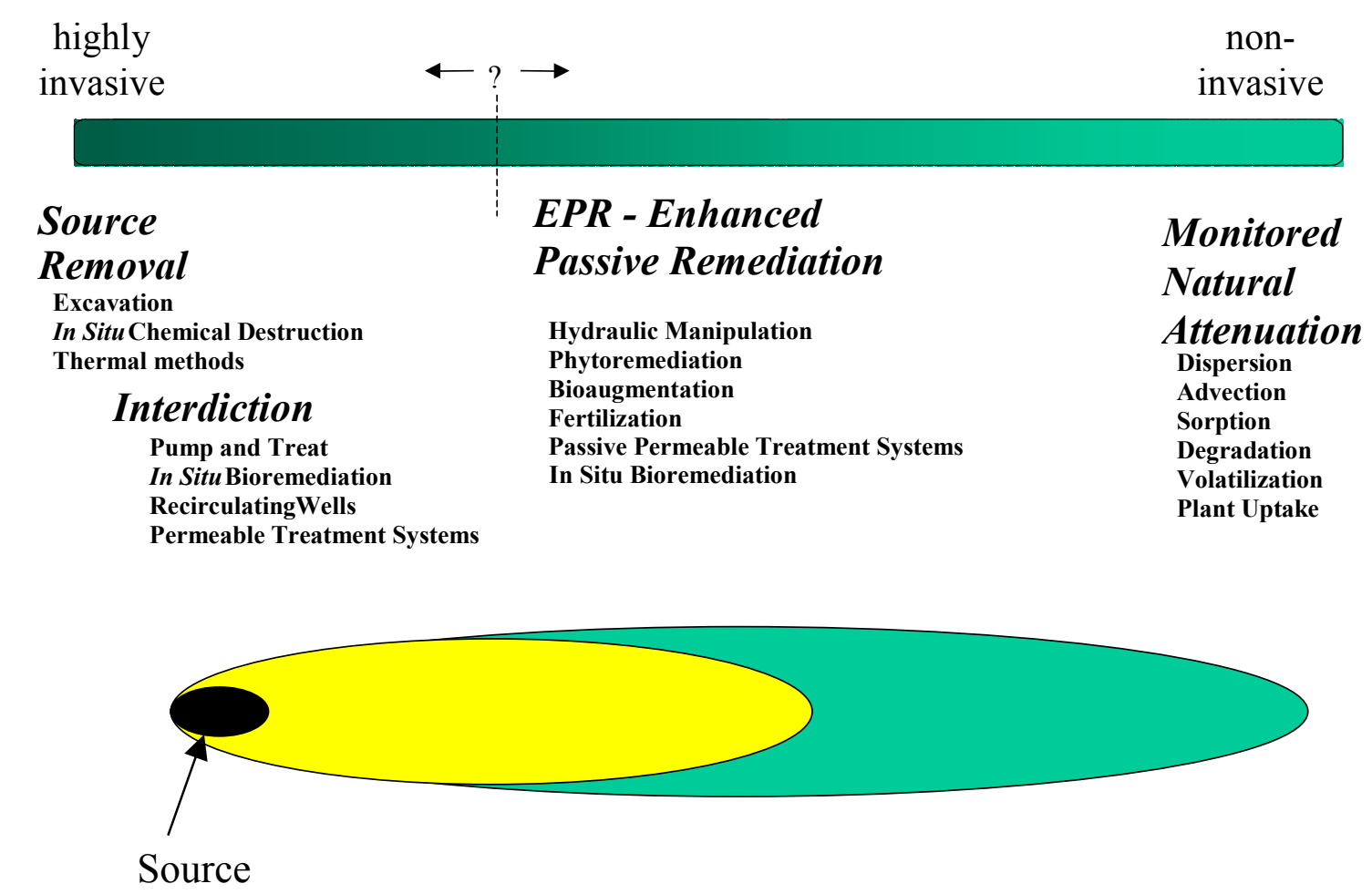

Figure 1 Continuum of Individual Remediation Technologies

Natural attenuation can be conceptualized as a balance between the delivery to and removal of contaminants from ground-water systems (Figure 2). If there is a certain mass of a non-aqueous phase (NAPL) contaminant $\left(\mathrm{N}_{\mathrm{m}}\right)$ in an aquifer, contaminant mass will dissolve over time and form a contaminant plume $\left(\mathrm{G}_{\mathrm{r}}\right)$. $\mathrm{G}_{\mathrm{r}}$ represents the mass discharge from the residual source and the equivalent mass loading to the plume. Once in solution, this dissolved contaminant will be transported by flowing groundwater and be subject to modifying processes (these processes, as well as $G_{r}$, are often expressed or converted into rates with units of mass/time). Within any "control" volume, contaminants will be diluted by dispersion $\left(\mathrm{D}_{\mathrm{r}}\right)$, discharged by advection $\left(\mathrm{A}_{\mathrm{r}}\right)$, destroyed by biodegradation and abiotic degradation $\left(B_{r}\right)$, slowed by sorption $\left(S_{r}\right)$, and removed by volatilization $\left(\mathrm{V}_{\mathrm{r}}\right)$, plant transpiration $\left(\mathrm{P}_{\mathrm{r}}\right)$ and similar processes (Figure 2$)$. The sum of the processes contributing dissolved contaminant mass to water (NAPL dissolution, desorption, etc) represents the contaminant loading part of the mass balance. Similarly, the sum of these removal/dispersive processes (except for advection) can be thought of as the natural attenuation capacity part of the mass balance within the control volume. Advection represents plume 
expansion beyond the control volume. While highly simplified, this conceptualization illustrates how these processes can interact in the subsurface (Figure 2).

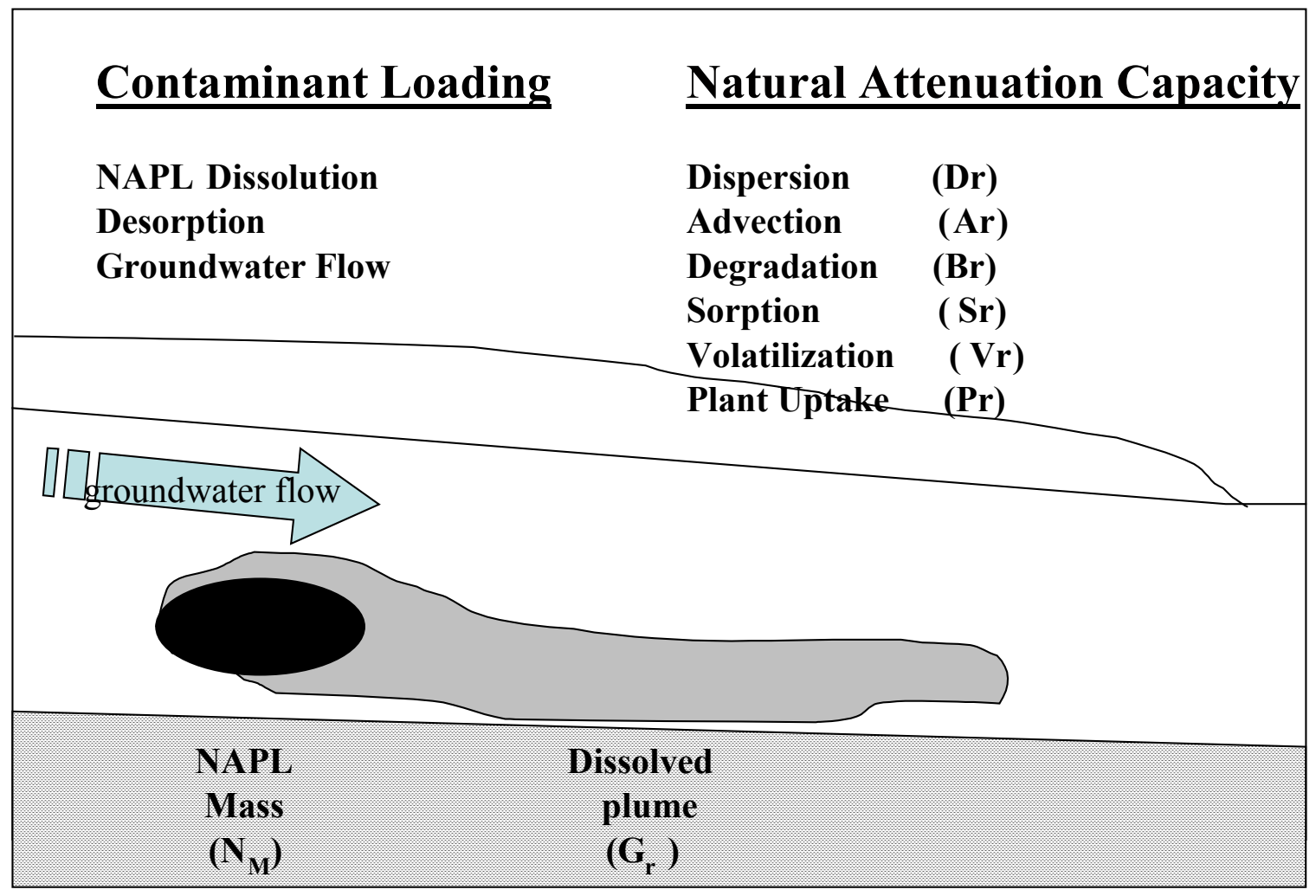

Figure 2 Schematic diagram of contaminant loading and natural attenuation capacity in ground-water systems.

For example if the contaminant loading to the plume $\left(\mathrm{G}_{\mathrm{r}}\right)$, is greater than the sum of the removal mechanisms

$$
\mathrm{G}_{\mathrm{r}}>\mathrm{D}_{\mathrm{r}}+\mathrm{B}_{\mathrm{r}}+\mathrm{S}_{\mathrm{r}}+\mathrm{V}_{\mathrm{r}}+\mathrm{P}_{\mathrm{r}}
$$

then the dissolved plume will expand over time (i.e., Advection is carrying contaminants beyond the control volume). Conversely, if contaminant loading is less than the sum of the removal mechanisms

$$
\mathrm{G}_{\mathrm{r}}<\mathrm{D}_{\mathrm{r}}+\mathrm{B}_{\mathrm{r}}+\mathrm{S}_{\mathrm{r}}+\mathrm{V}_{\mathrm{r}}+\mathrm{P}_{\mathrm{r}}
$$

then the dissolved plume will contract over time. Finally, if contaminant loading is equal to the sum of the removal mechanisms

$$
\mathrm{G}_{\mathrm{r}}=\mathrm{D}_{\mathrm{r}}+\mathrm{B}_{\mathrm{r}}+\mathrm{S}_{\mathrm{r}}+\mathrm{V}_{\mathrm{r}}+\mathrm{P}_{\mathrm{r}}
$$


then the dissolved plume will be stable. The regulatory basis for evaluating MNA as a remedial strategy often rests upon whether a contaminant plume is stable, expanding, or contracting.

As implied in the conceptual model, the first step in the mass balance approach is developing a clear and agreed definition of the target plume volume and then determining the balance between contaminant loading and the natural attenuation capacity of that given vadose-ground-water system (Figure 2). Under this scenario, the feasibility of MNA is related to the contaminant loading $\left(\mathrm{G}_{\mathrm{r}}\right)$ combined with the composite "rate" of the relevent attenuation processes. Similarly, the overall timeframe of remediation is related to the source quantity $\left(\mathrm{N}_{\mathrm{m}}\right)$ combined with the composite "rate" of the relevent attenuation processes. If the attenuation capacity is too low for plume stability, options for EPR can be examined. There are many complexities in implementing this approach. For example: what is the best method to account for spatial and temporal variation in the attenuation capacities? and what is the best approach to quantify these rates in a simple fashion for practical use at real-world sites? These topics are the basis of some of the key science and technology targets for advancing MNA and EPR.

This conceptualization also illustrates another characteristic of evaluating natural attenuation. Except in cases where the system itself provides unambiguous information that the plume is stable or shrinking (a direct measure of the balance), then the individual contributors to the balance need to be determined.

The different components contributing to the mass balance of a given site cannot necessarily be directly measured. Rather the measured components are indirect indicators of the attenuating mechanisms.

Each component of equations 1-3 is a definable quantity (Figure 2), but not necessarily one that can be directly measured. Because of this, actual evaluations of natural attenuation must rely on indirect measurements and indicators for each component of the mass balance problem. This is analogous to an artist who must draw a portrait from the shadow of the person being sketched. Drawing an accurate image from a shadow is much more challenging then observing the subject directly. The indirect nature of the evidence used to assess MNA imposes similar difficulties and challenges to the environmental professional. A clear extension of this discussion is the concept that new technologies which provide more direct measures of the key processes are potentially beneficial in illuminating the picture.

The indirect nature of the evidence used to assess natural attenuation capacity can be illustrated using biodegradation processes as an example. Biodegradation processes of chlorinated ethenes are highly complex, and consist of reductive, fermentative, oxidative, and cometabolic processes. Furthermore, one or more of these processes may occur simultaneously, sequentially, or not at all as contaminants are transported along the hydrologic gradient. Direct measurements of which processes occur at particular points in a system, and at what rate these processes occur, are not presently technically feasible. Thus, a series of indirect indicators are used to assess which biodegradation processes occur and at what rate they occur. The most common of these indirect 
indicators is concentrations of dissolved oxygen in groundwater. If dissolved oxygen concentrations are higher than about $2 \mathrm{mg} / \mathrm{L}$ in a system, then reductive dechlorination of TCE will not occur efficiently whereas oxidative degradation of vinyl chloride will be highly efficient. In the same manner, the presence of methane (methanogenesis), sulfate/sulfide (sulfate reduction), ferrous iron (Fe(III) reduction), nitrate/nitrous oxide (nitrate reduction), and concentrations of dissolved hydrogen are indirect indicators of which biodegradation processes (reductive dechlorination, anaerobic oxidation, etc) are occurring, what their spatial distribution is, and how rapidly these processes occur. There is also an interaction between the different attenuation terms. Sorption stabilizes a plume causing it to grow and contract more slowly and providing more residence time for destruction processes. The interactive benefits of sorption, or the potential to adversely impact a site by releasing sorbed contaminant during EPR, are clear examples of interactive impacts. Assessing how much biodegradation is contributing to the overall natural attenuation capacity is an indirect, highly interpretive process. These concepts are not unique to biodegradation, but also hold for each individual component of natural attenuation capacity (Figure 2). The indirect nature of the methods used to assess natural attenuation capacity makes evaluating MNA an inherently complex procedure.

Finally, another source of complexity stems from the heterogeneous nature of groundwater systems. Biodegradation rates, sorptive capacity, and volatilization can differ by orders of magnitude over distances of just a few centimeters. Rates of groundwater flow can vary over several orders of magnitude in time and space. Due to this inherent, ubiquitous, and unavoidable heterogeneity, it is difficult to obtain representative estimates for each component of natural attenuation capacity at real sites. As a result, monitoring tools that integrate processes over an appropriate scale represent another potentially high priority research and technology development target.

The technical working group strongly supported the conceptualizing of MNA/EPR as a balance between contaminant loading and attenuation capacity. MNA/EPR performance evaluation based on a balance of attenuation capacity and loading is intuitive and powerful. It provides an understandable organizing framework that is compelling and yet flexible enough to handle real world complexities and technically challenging situations. The ideas can be supported by the multiple lines of evidence that are necessarily embodied in MNA and EPR protocols. Importantly, when framed in terms of attenuation capacity and loading, the confusion that is often associated with long lists of required and optional analytes can be overcome. Why are certain parameters collected? How should they be used? The loading-capacity balance concept is also adaptable to each site. At some sites, unambiguous measurement of plume stability or shrinkage provides a field scale verification that attenuation capacity is greater than or equal to loading and little further evidence will be necessary. For sites where there is more uncertainty, more challenges or where enhancements to MNA are being considered, additional data and complexity can be added as needed - always within the framework. The concept is also portable in terms of modeling - simple and complex models can easily generate results that explicitly allow the balance to be calculated. Finally, and most importantly, an approach that balances contaminant loading and attenuation capacity is exactly the type of tool needed to link MNA/EPR into the overall planning necessary at a contaminated site. 
Recent scientific studies for source remediation are examining performance in terms of mass discharge from the source to the plume. The mass discharge from the source is the initial contaminant loading to the plume and provides a direct link to support decision making and answering the difficult questions that often arise at chlorinated solvent sites. How much source needs to be removed to integrate with the overall long term goals for the site? When can an active treatment system be turned off? How can the mass balance approach be integrated into an overall strategy to protect receptors and reduce risk (e.g., account for exposures through all routes - indoor air, etc.)? The concept also suggests a few changes in the types of data that would be most useful. In source removal or destruction efforts, for example, a measurement or estimate of the reduction in mass discharge, rather than total mass, would be of value. All of the efforts that are currently being invested in estimating mass discharge from the source can be directly applied to improving environmental decisions. Similarly, understanding the flux or loading in different portions of a plume will provide a powerful tool for determining the size of an active plume treatment and the appropriate conditions that would justify transition to MNA/EPR. 


\subsection{Key Definitions}

There are several terms used consistently throughout this document. To help the reader, these terms are defined in this section. Several of these terms, such as MNA, have been defined by other fields, such as the engineering field, or by federal agencies, such as the US EPA. It is our goal to be true to the intent/spirit of the definition. However, in some cases we have changed the words to show the linkage with the purposes and goals of this project.

Attenuation Capacity - The sum of all physical, biological, and chemical processes serving to disperse, biodegrade, chemically transform, immobilize, or permanently sequester chlorinated ethenes in a ground-water system. Processes that contribute substantially to attenuation capacity include advection, hydrodynamic dispersion, biodegradation, sorption, volatilization, abiotic transformations, and uptake by plants. Capacity and loading are normally measured in units such as $\mathrm{Kg} / \mathrm{year}$.

MNA - Monitored Natural Attenuation - Managing all or part of a contaminant plume in soil and groundwater by utilizing the existing decontamination and attenuation mechanisms of the natural system and by documenting the resulting attenuation capacity. MNA is a viable alternative if the attenuation capacity equals or exceeds the potential contaminant loading from the plume source and/or if the plume is shown to be stable or shrinking and will not impact a receptor. Consistent with the US EPA definition there is no "no human intervention" in the attenuation and remediation processes.

EPR - Enhanced Passive Remediation - Managing all or part of a contaminant plume in soil and groundwater by initiating and/or augmenting natural and sustainable decontamination and attenuation mechanisms and by documenting the resulting attenuation capacity. EPR is a viable alternative if the resulting attenuation capacity equals or exceeds the potential contaminant loading from the plume source and/or if the plume is shown to be stable or shrinking and will not impact a receptor. EPR is based on the definition of MNA but allows for "human intervention" that will result in natural and sustainable mechanisms.

Active Remediation - Managing all or part of a contaminant plume in soil and groundwater by active or frequent intervention using processes that are not naturally sustainable over the time frame required for plume remediation.

Source Treatment - Destruction or enhanced removal processes for high concentration contaminant source zone(s).

Source Excavation - Physical removal followed by isolation or surface treatment of contaminant source zone materials. 


\subsection{Historical and Regulatory Perspective}

\subsection{Historical Background}

As noted above, natural attenuation processes occur at every site. Recognition, evaluation and reliance on these processes for remediation and final polishing of contaminated sites has been limited but has steadily increased over the past twenty years. A critical component of advancing the technical bases for the use of MNA/EPR is documenting this historical development and assessing, using a retrospective survey, what is working and where there are barriers to implementation. Early variants took the form of "alternate concentration limits" (ACLs) or "mixing zones". These concepts provided a useful tool for relatively low risk sites and were based on modeling - the goal was to calculate a target concentration in the plume that would not result in an exceedence of an applicable or appropriate standard at an agreed exposure location. The guidelines for ACL modeling did not emphasize the full range of natural attenuation and remediation mechanisms. As a result, scientists, regulators and policymakers began a process to develop improved guidelines that would encourage responsible and disciplined use of remediation and site management strategies that rely on natural processes. These efforts were squarely focused on natural attenuation processes and led to the definition and understanding of the term "MNA" (US EPA, 1998 and US EPA, 1999). The efforts were customized, as appropriate, to major classes of common contaminants. This allowed the most applicable mechanisms to be identified and specific guidelines and protocols to be established for the most widespread problems. Importantly, the "M", or monitoring, aspect of MNA was a key factor and was critical to the acceptance and success of MNA for all contaminants as various protocols were developed. It is particularly instructive to examine the timing of protocol development efforts for various classes of organic contaminants.

Figure 3 provides a timeline for the technical protocol and regulatory protocol development for monitored natural attenuation (MNA) for petroleum hydrocarbons and for chlorinated solvents. It is clear from the timeline that development of MNA for petroleum hydrocarbons, because the underlying processes are inherently more robust and simple, occurred earlier than the development of MNA for chlorinated volatile organic contaminants (CVOCs). Moreover, MNA of petroleum hydrocarbons is now widely accepted and used. Protocol development for CVOCs has proven to be more complex and the viability, robustness and utility of the CVOC protocols has not been documented. How have they worked? How many sites have they been applied to? What percentage of those sites met the requirements? What parts of the protocol have been most useful in practice? What parts of the protocol have been least useful? Obtaining answers to these types of questions is an absolute requirement for any effort whose goal is to build on and contribute to this historical process and to the positive evolution of MNA/EPR. The answers to key questions are key input that will help determine the most promising research and regulatory path. Of particular interest are experiences in the period since 1998/1999 - after the US EPA regulatory protocols and directives were released. A few historical evaluations have 
been performed - normally covering a case study or a few sites. To assist in developing a target list of critical science and technology needs and issues to support advancing CVOC protocols, a general historical survey was performed.

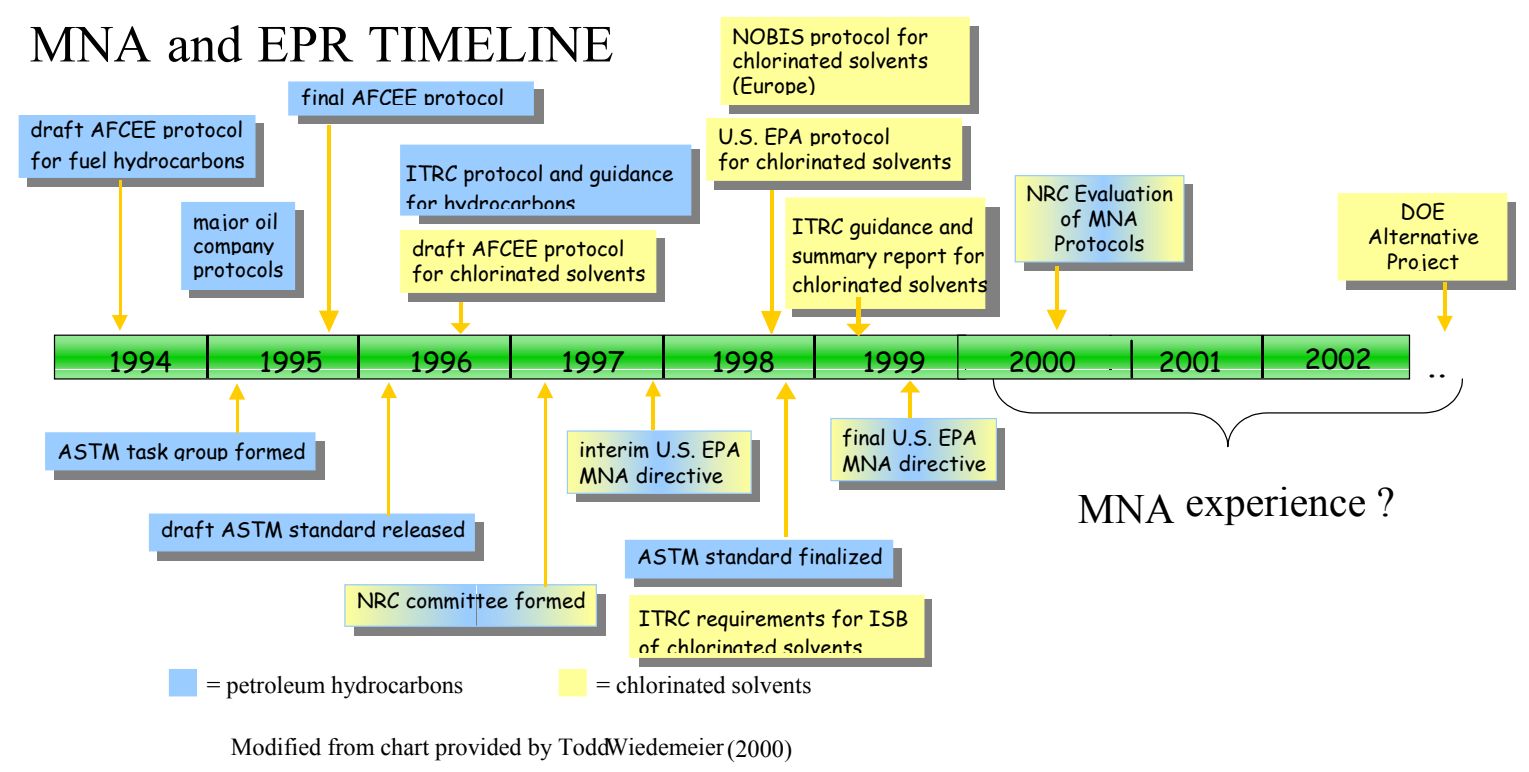

Figure 3 Recent Historical Development of MNA/EPR for Petroleum Hydrocarbons and Chlorinated Solvents (modified from Wiedemeier and Barden, 2002)

\subsubsection{The Survey}

A Historical Analysis of MNA survey was developed to gain a better understanding of the application of MNA at sites affected with chlorinated solvents. The survey sought to provide insights into the remediation professional's general experience with MNA and to gather site-specific data regarding the implementation of MNA as a remedy of a particular CVOC plume. The survey was distributed to approximately 230 remediation professionals in industry, government, and academia with experience in the field of MNA.

The survey was divided into two parts: part A) general request for information about MNA experiences and B) more detailed data on a specific site. Part A consisted of four questions and attempted to gather high level information. How many sites? How successful? Typical size? Typical cost? Part B consisted of 39 questions requesting data for a specific plume where MNA was applied as the remedy, either solely or as a component of other active treatment. These questions explored many topics including the types of monitoring and modeling, the status of the effort, and the like. The specific questions that were asked in both parts are noted on the graphs of the results. 


\subsubsection{The Survey Results}

Survey data was received from 30 individuals for a total of 178 waste sites; all respondents provided Part A data, and site-specific data was received for 42 individual chlorinated solvent plumes. Data from Part A and Part B are summarized in figures 4a - 411 (these figures are presented after the conclusions and regulatory discussion). Each figure is labeled with the relevant question or topic and the results provided interesting and useful insights as summarized below. The figures are provided to establish visual trends in the data rather than establishing any statistically significant results.

The results presented in this section are preliminary and were used by the Technical Working Group during their preparation of this document. The final results are presented in the supplemental document "Historical and Retrospective Survey of Monitored Natural Attenuation: Lines of Inquiry Supporting Monitored Natural Attenuation and Enhanced Passive Remediation of Chlorinated Solvents," WSRC-TR2003-00333. The final results were consistent with the initial results reported below.

\subsubsection{Key Conclusions from the Survey}

\section{Part A:}

- MNA was determined to be feasible as a remedy at over $75 \%$ of the sites where the application of MNA was evaluated (36\% sole remedy, $46 \%$ with other treatment). Importantly, MNA was determined to be infeasible at about $23 \%$ of the sites and follow-up contacts will be conducted to elicit the reasons.

- At sites where MNA is used with an active treatment, the active treatment is still in operation at approximately $72 \%$ of those sites.

- The average cost of the entire initial MNA study was reported to be about $\$ 188,000$ and results ranged from $\$ 10,000$ to $\$ 750,000$.

- The average annual cost for monitoring an MNA site was found to be $\$ 32,000$ with a range of $\$ 3,000$ to $\$ 150,000$.

- Nearly half of the respondents reported that the typical size of a chlorinated solvent plume where MNA is utilized in the remedial scheme is 10 to 50 acres, while $29 \%$ and $25 \%$ reported the average size to be less than 10 acres and greater than 50 acres, respectively.

Part B:

- MNA is used as a remedy at a variety of industrial sites with a broad range of processes.

- The 1998 US EPA protocol (US EPA, 1998 and 1999) was most often referenced as the guideline for MNA implementation (36\%). Notably, almost $29 \%$ used a site 
specific protocol. Other protocols used as the basis for the reported sites included: $12 \%$ state protocol, $19 \%$ other, and 5\% National Research Council (NRC) MNA review (NRC, 2001).

- Almost $70 \%$ of respondents stated that anaerobic degradation is the primary natural attenuation process occurring in the plume, while the remaining attenuation processes each accounted for less than $7 \%$

- A variety of geochemical indicators are reportedly used to assess MNA, but over $90 \%$ rely on the presence of biodegradation daughter products.

- A variety of tools were used to support MNA, including conceptual models, analytical models, and mass flux calculations. About $19 \%$ of the respondents reported that none of these approaches was used in implementing MNA.

- Computer models of various types were used to evaluate MNA at 57\% of the sites. The most common model used was BIOCHLOR.

- The timeframe for remediation was estimated at over $60 \%$ of the sites, and most (44\%) expect remediation goals to be achieved in 11 to 20 years. This result was surprising to the technical working group (TWG) and suggests that current conceptual models of MNA may be overly optimistic.

- For half the plumes reported, TCE is the original contaminant released. PCE follows at 33\% of sites, with releases of cis-DCE, vinyl chloride, 1,1,1-TCA and 1,2-DCA also reported.

- Most plumes evaluated have a maximum length of 1000 to 5000 feet, an area of 1 to 10 acres.

- $34 \%$ of the MNA sites were less than 100 feet from a downgradient discharge.

- The respondents classified the plumes as stable or shrinking at $52 \%$ of the sites. Interestingly, the plumes were classified as expanding for $10 \%$ of the sites and discharging for $22 \%$ of the sites.

- The most important lines of evidence in documenting MNA were concentration decreases and geochemical footprints".

\subsection{Regulatory and Peer Review Perspective}

Several key milestones where regulatory protocols and guidance directives, as well as independent scientific evaluations, were completed are evident in the MNA/EPR timeline presented above (figure 3 ). These critically important enabling documents are discussed below as the central context to support future science and technology development / prioritization efforts.

\footnotetext{
"Geochemical footprint" is a term that was widely introduced by the National Research Council of the National Academy of Sciences in their independent review of US EPA's MNA protocol and directive. The term relates to spatial mapping of MNA related parameters (e.g., sulfate, nitrate, oxygen, daughter products) and documentation that the patterns are consistent with MNA conceptual models and goals.
} 
The first step in the decision-making process for use of MNA/EPR at a particular site is to determine the regulatory program or programs under which the cleanup will be conducted and to establish what the cleanup goals are for that site. The cleanup of many sites, including those at federal sites, is overseen jointly by US EPA and the responsible state environmental agency. The specific roles and responsibilities of the regulator agencies are usually agreed to and documented in agreement documents signed by all parties involved. Cleanup goals will typically include both intermediate (shorter-term) goals as well as the ultimate (final and/or longer-term) cleanup goals for the site. For federal cleanup programs, such as RCRA Corrective Action or Superfund, intermediate goals will typically include preventing contaminants from reaching human and environmental receptors, controlling sources, preventing plume migration, and other goals needed to protect human health or the environment. Ultimate goals for these federal programs will typically include returning the contaminant plume to cleanup levels appropriate for potential beneficial uses of the groundwater, which in most cases are federal and state drinking water standards. (Note that the term "cleanup goal" can sometimes be misinterpreted as a desired target rather than a regulatory requirement. For this reason, the term remedial action objective (RAO) is used in US EPA Superfund guidance when referring to a required outcome. In this document, "cleanup goal" is intended to have the same meaning and is used in place of the term "remedial action objective.")

US EPA's Office of Solid Waste and Emergency Response (OSWER) finalized the policy directive: "Use of Monitored Natural Attenuation at Superfund, RCRA Corrective Action, and Underground Storage Tank Sites" in April 1999, as shown on the timeline in Figure 3. In developing this US DOE document, the authors have assumed that federal and state cleanup programs will consult and follow the guidelines established in the US EPA policy when evaluating proposals for use of MNA as a component of the remedy at a particular site. For this reason, users of this document are encouraged to become familiar with the US EPA directive. Also, key elements of the US EPA policy directive are highlighted below:

- MNA should not be considered a "no action," "default" or "presumptive" remedy.

- Use of MNA should not result in plume migration or impacts to receptors that would be "unacceptable to the overseeing regulatory authority."

- Selection of MNA should be based on thorough site characterization and comparison with other cleanup methods.

- In general, MNA should be used with other cleanup measures or as follow-up to such measures (e.g., source control, pump and treat [P\&T] of plume hot spots).

- The timeframe for MNA to attain site-specific cleanup goals should be "reasonable" when compared to the time required by active methods. 
- A contingency remedy should be included when selection of MNA was based mostly on predictive analysis.

- Progress of an MNA remedy should be carefully monitored.

- A remedy is not considered complete until cleanup objectives have been met.

A review by the National Research Council of the National Academy of Sciences (NAS, 2000) generally supported the concepts of MNA as articulated by US EPA. Some of the key chlorinated solvent related conclusions emerging from this independent effort, and some of the alternative recommendations included:

- Community groups are generally wary of MNA as a remedy. Stakeholders often want to be convinced that dilution is not the primary process and that some form of contaminant destruction is in place.

- Community groups should be involved early in the decision process.

- Decreases in concentration may not provide sufficient basis for selecting MNA. Data documenting attenuation mechanisms are also needed.

- Greater level of effort is needed to document MNA at complex sites.

- A long-term monitoring plan, including a contingency plan, should be specified for every MNA remedy.

- Future protocols should minimize use of highly simplified general "scoring systems" for decisions on MNA remedies.

It is clear, from the historical survey and from the regulatory and review documentation that MNA is a promising tool and that there has been significant success in implementation since the publication of protocols in 1998 and publication of an OSWER Directive in 1999. The retrospective analysis provided some clear indications of approaches that have worked, approaches that have not worked, and the regulatory framework that govern any MNA implementation. This information, in turn is a critical factor in determining and prioritizing future science and technology efforts.

Figure 4 Graphs of Historical Survey Results (follow) 


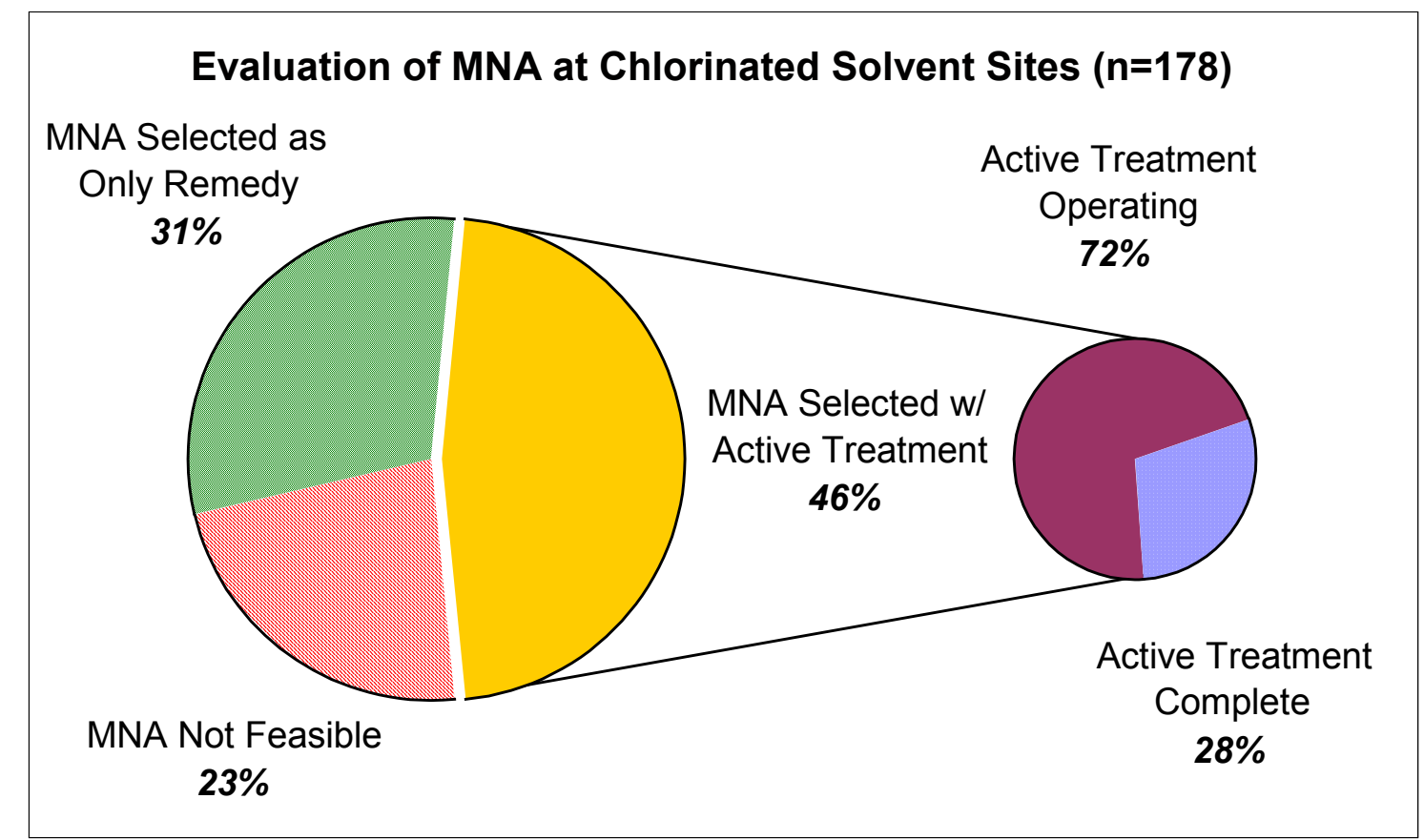

Figure 4a

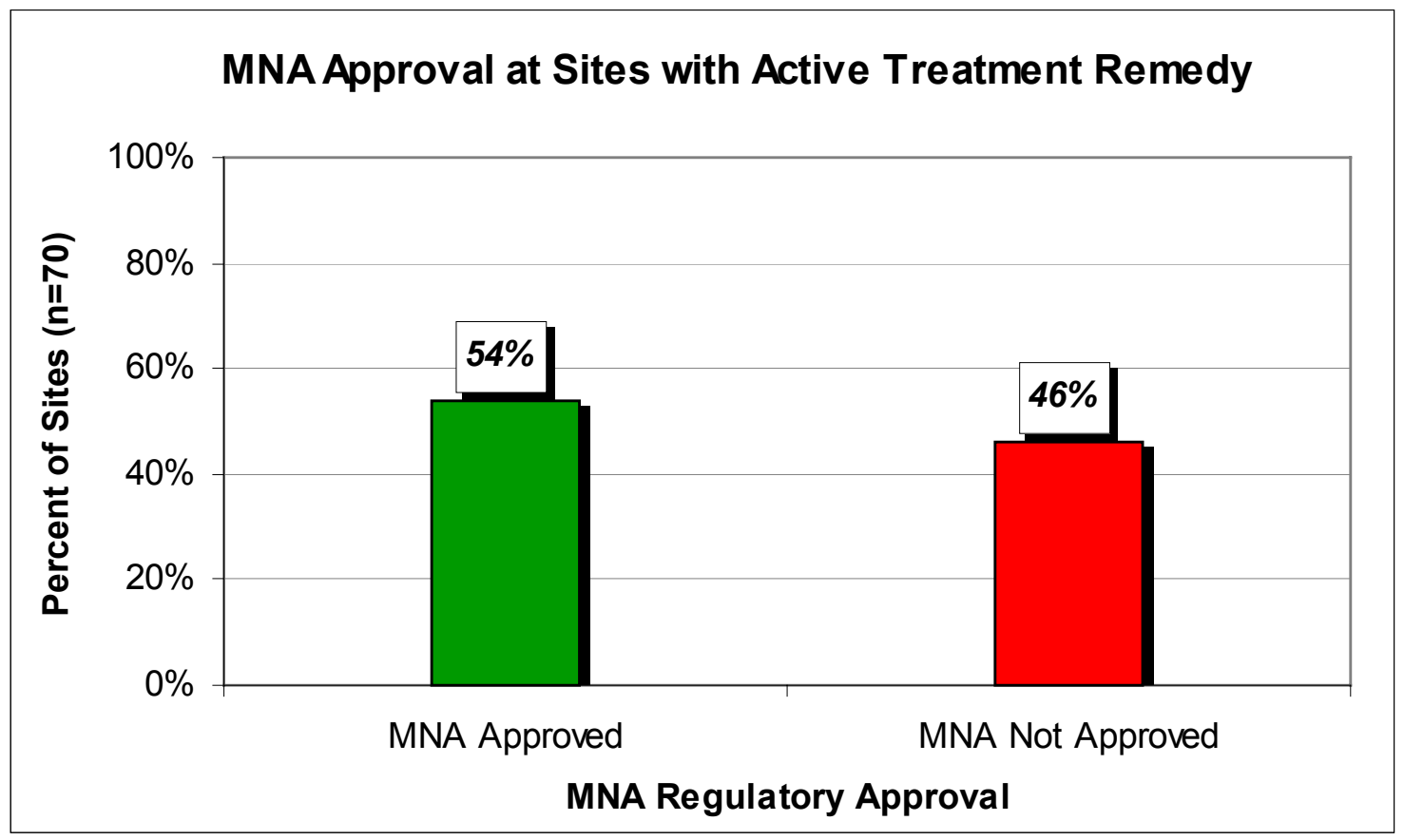

Figure $4 b$ 


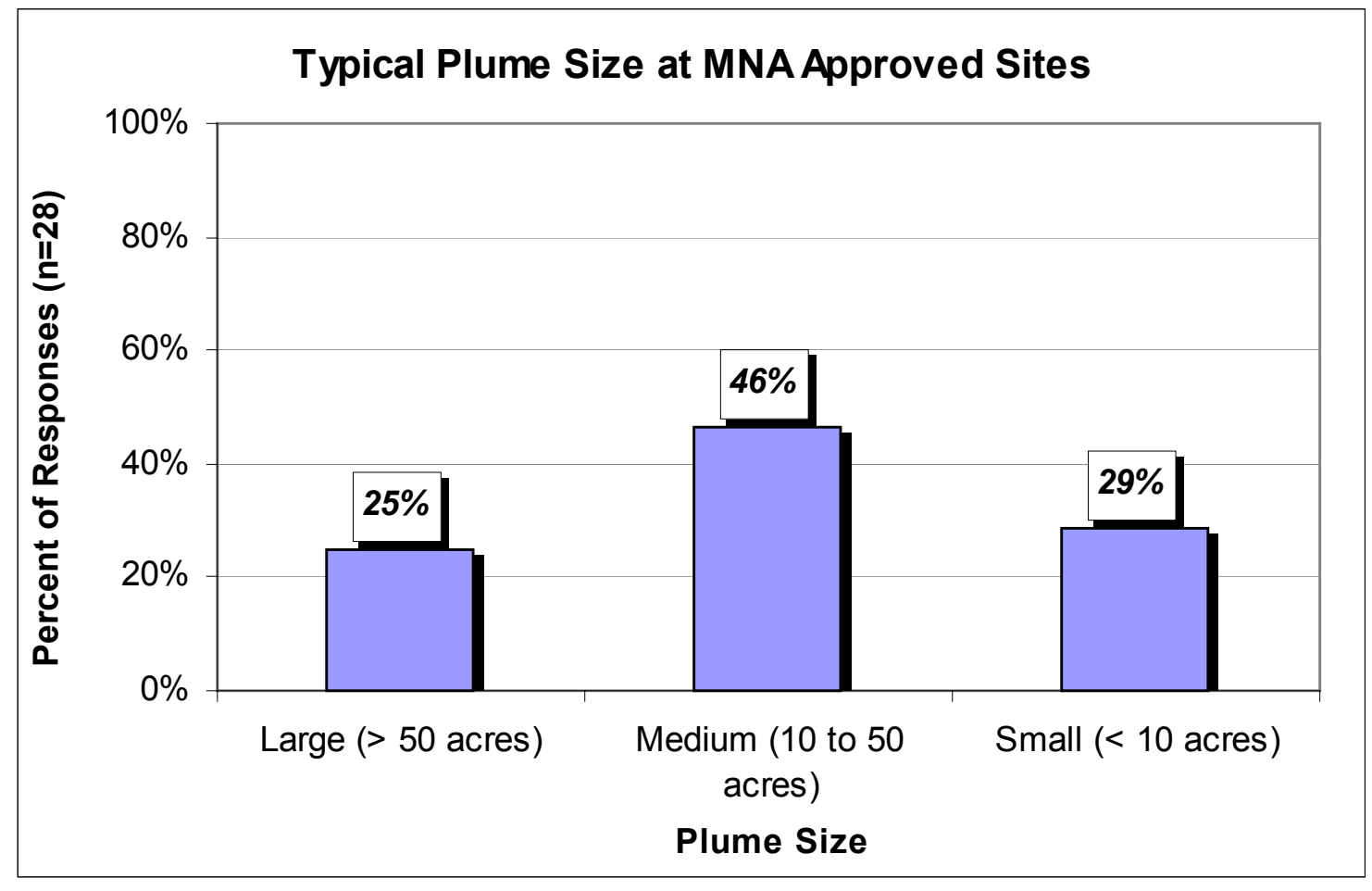

Figure 4c

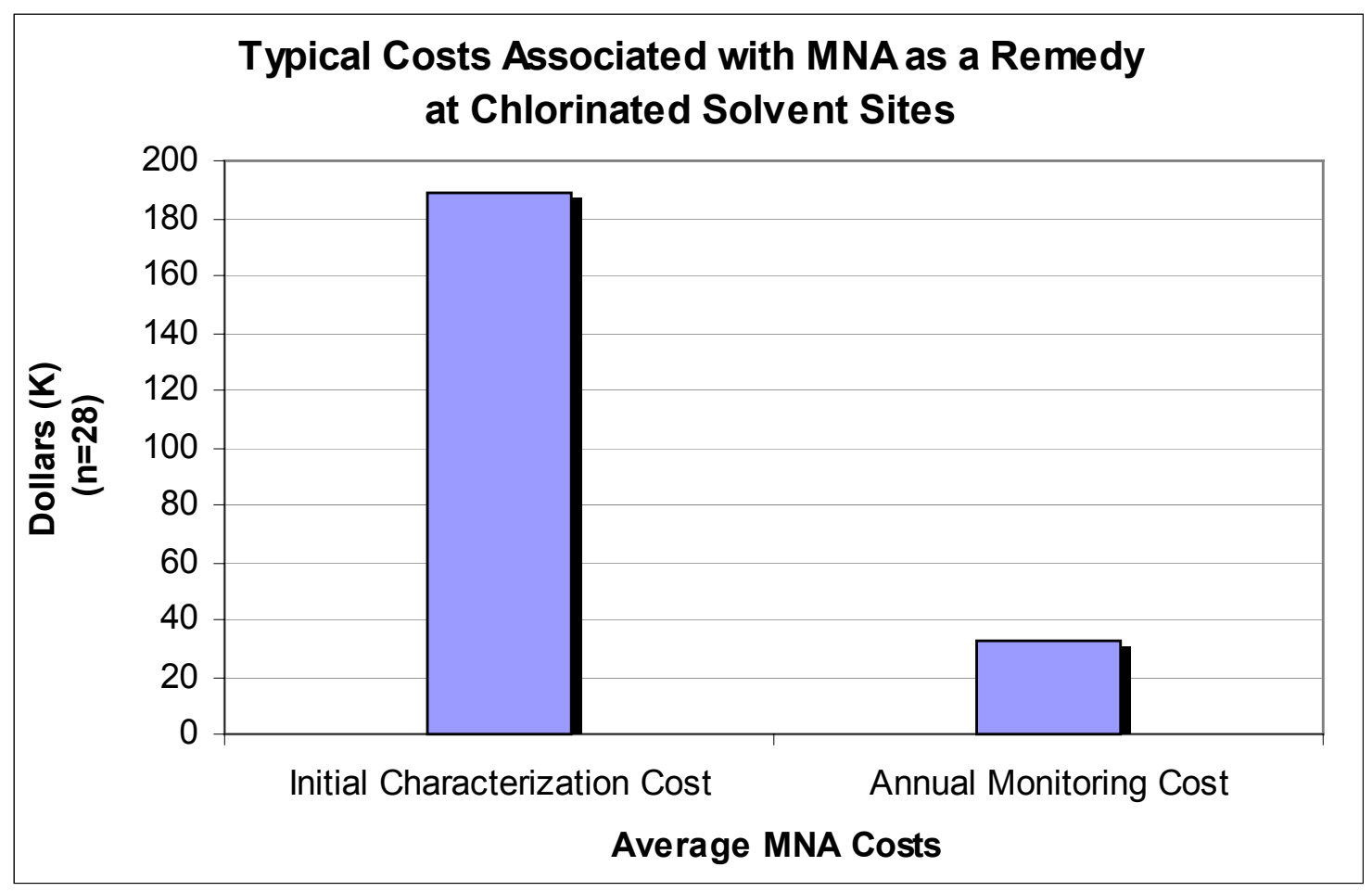

Figure 4d 
WSRC-TR-2003-00328

February 19, 2004

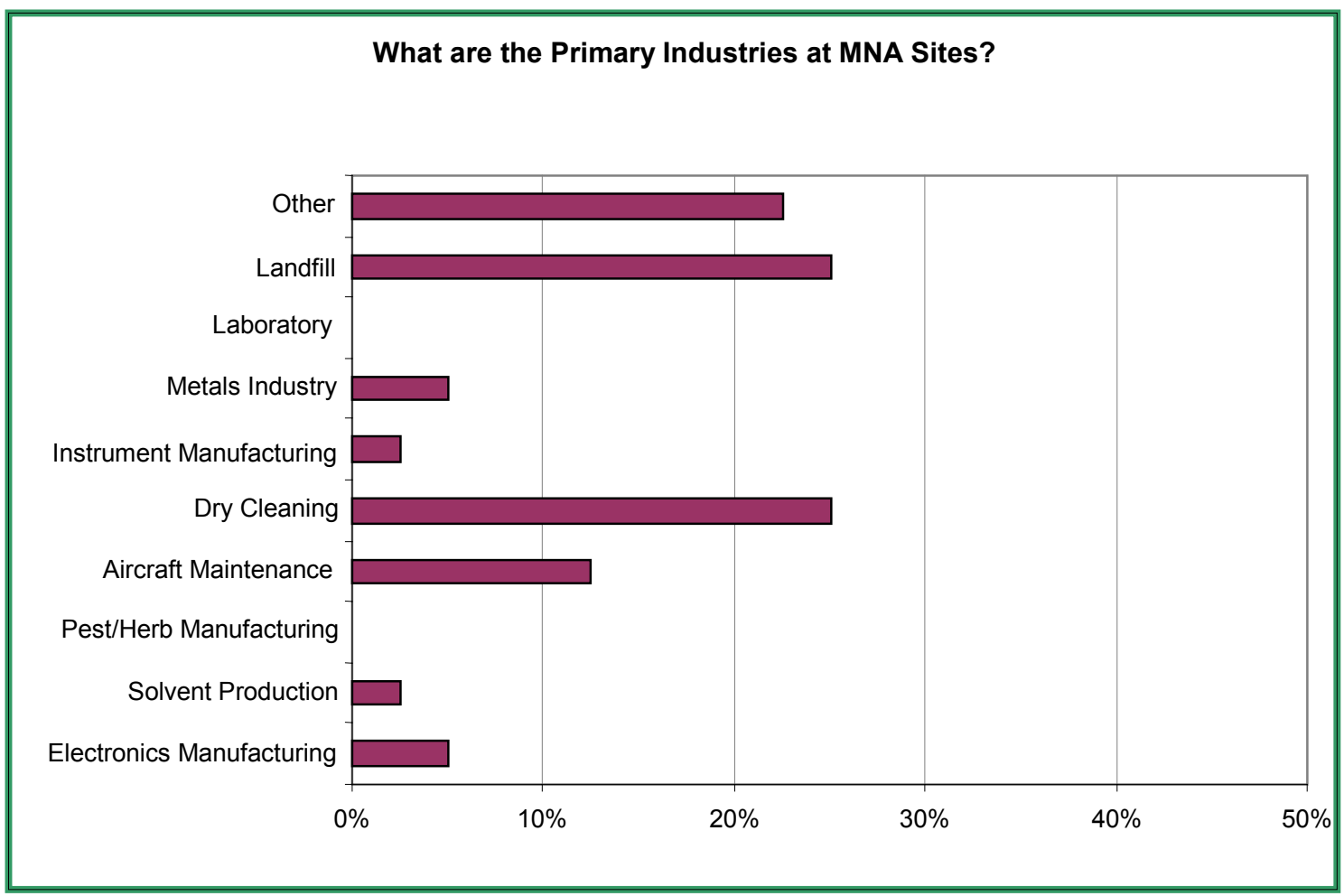

Figure 4e

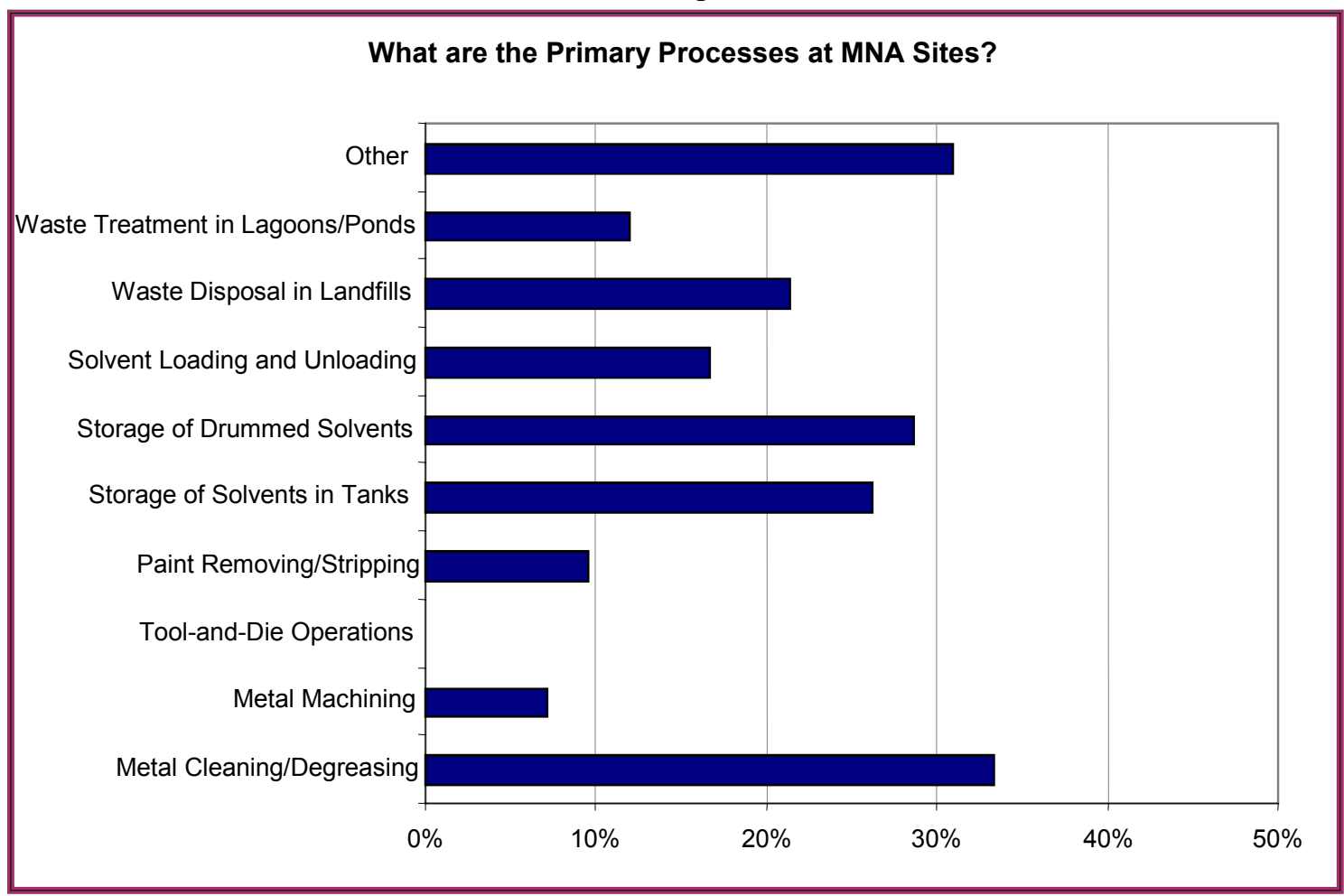

Figure 4f 


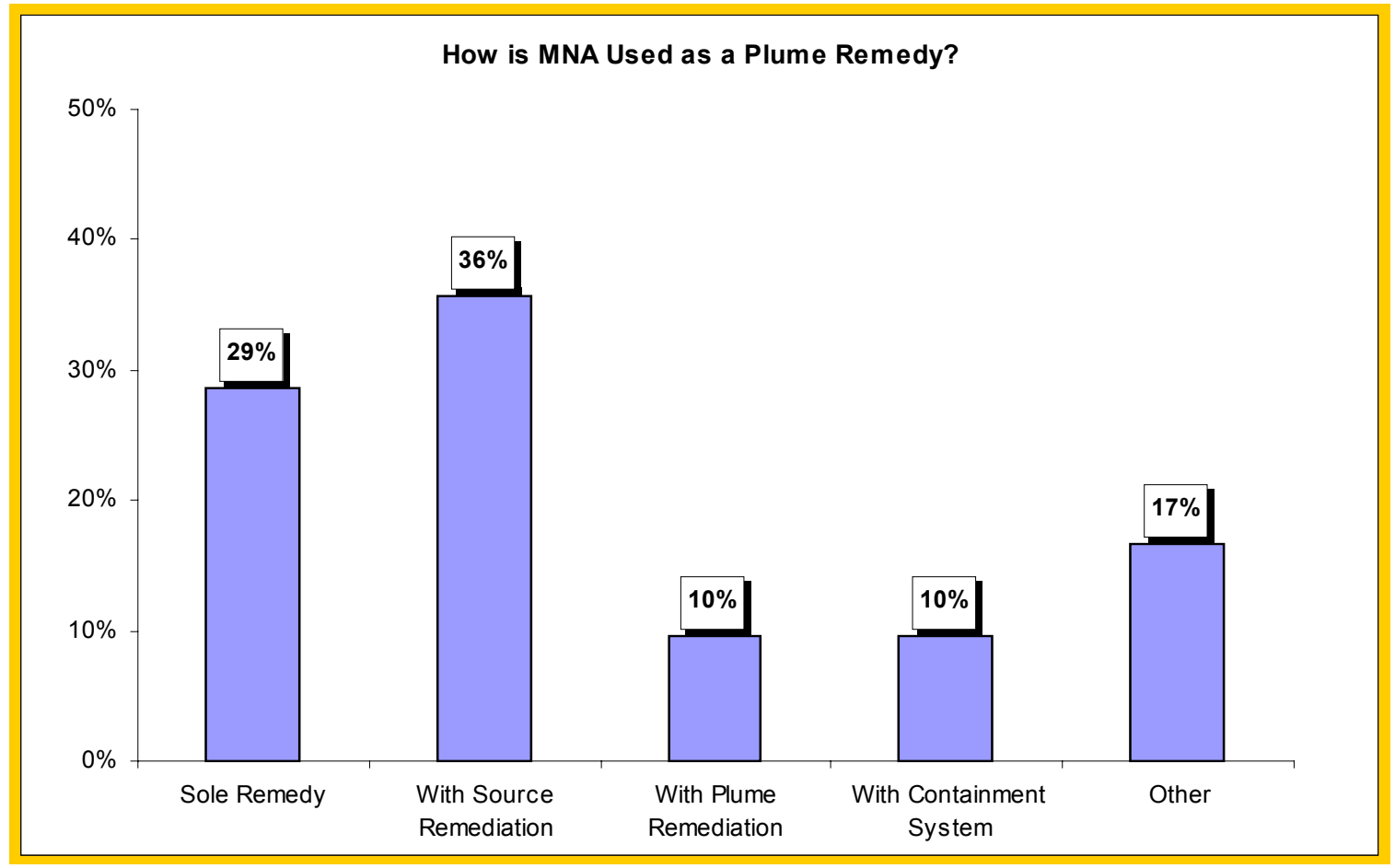

Figure 4g

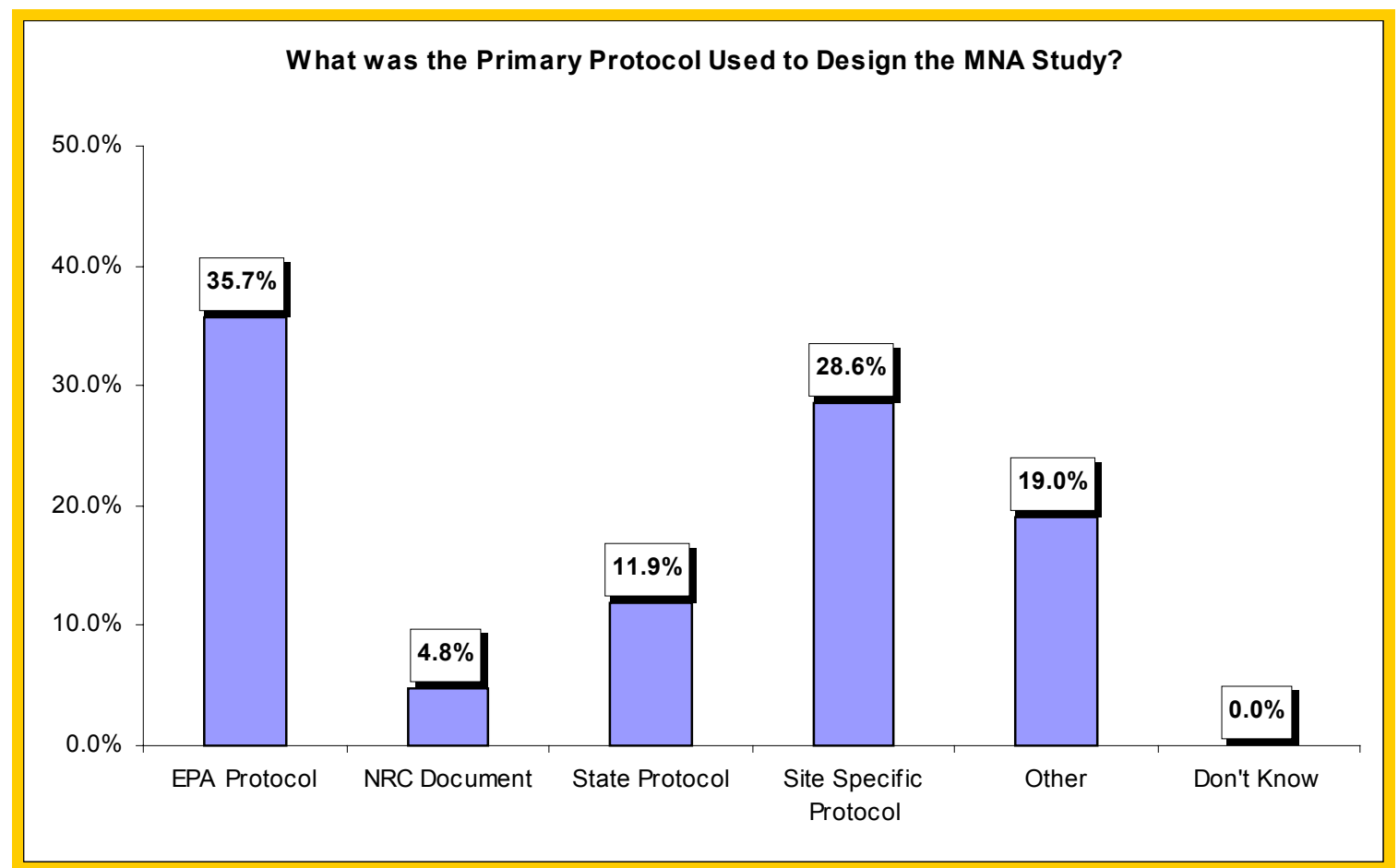

Figure 4h 


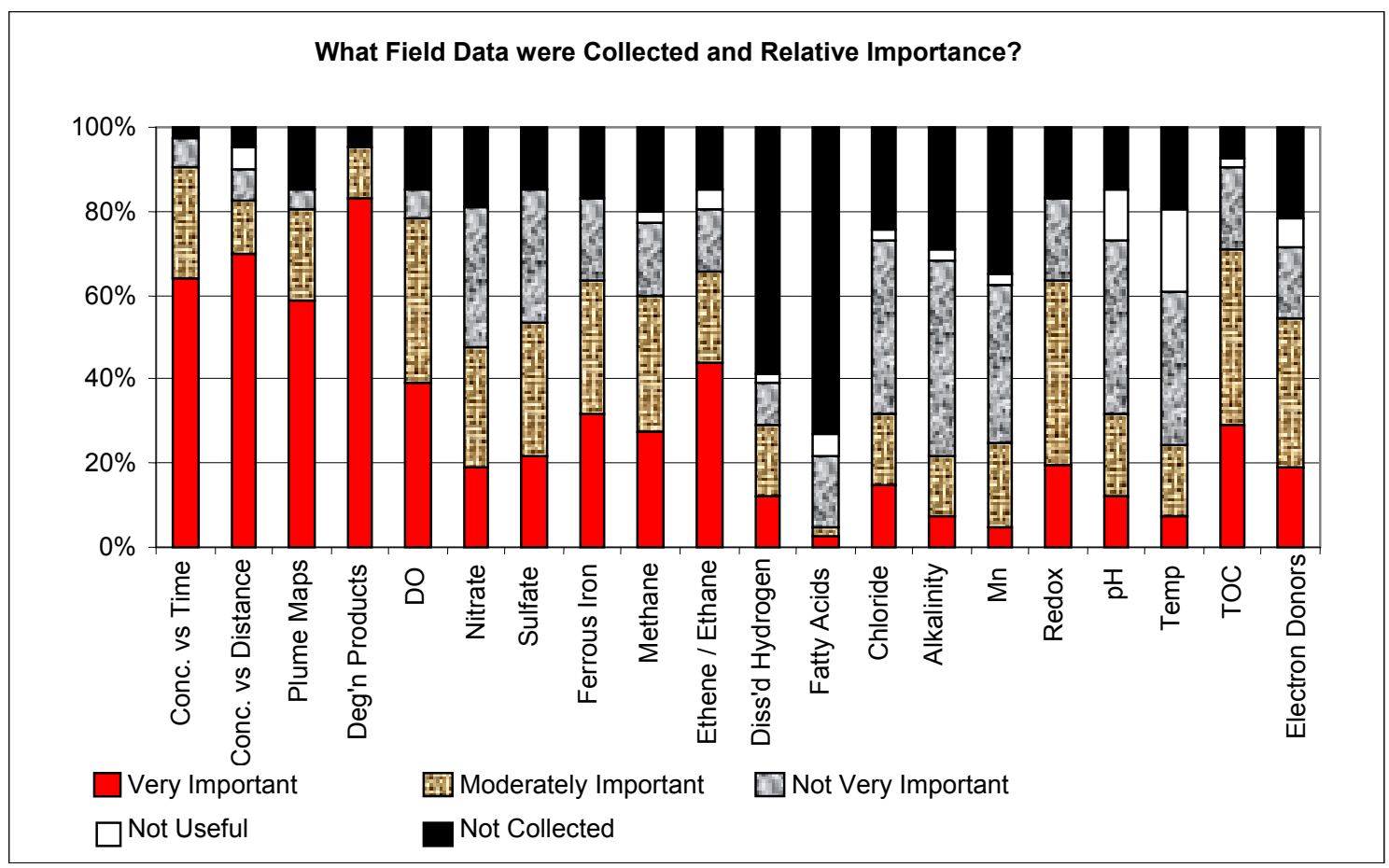

Figure 4i

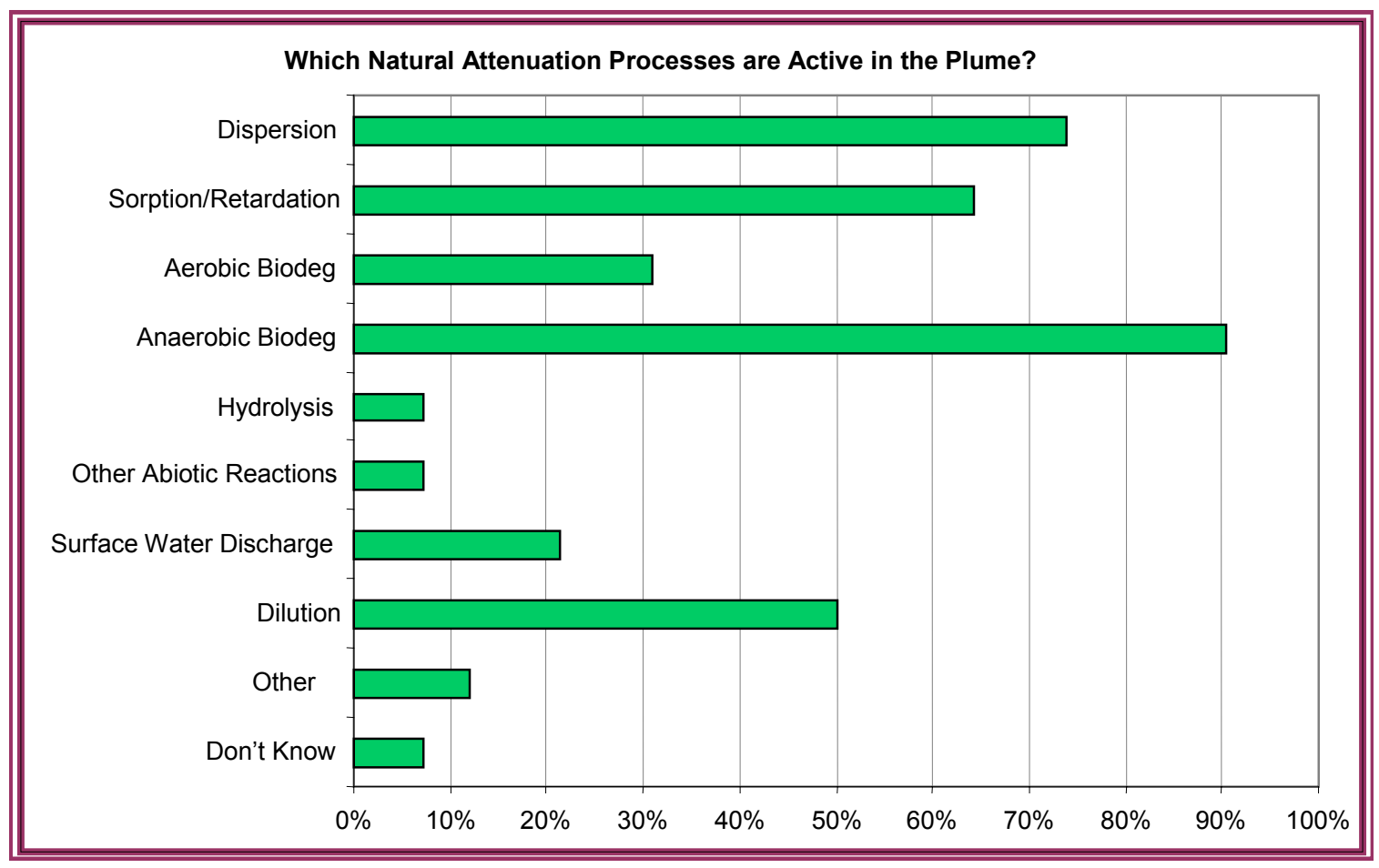

Figure $4 \mathrm{j}$ 


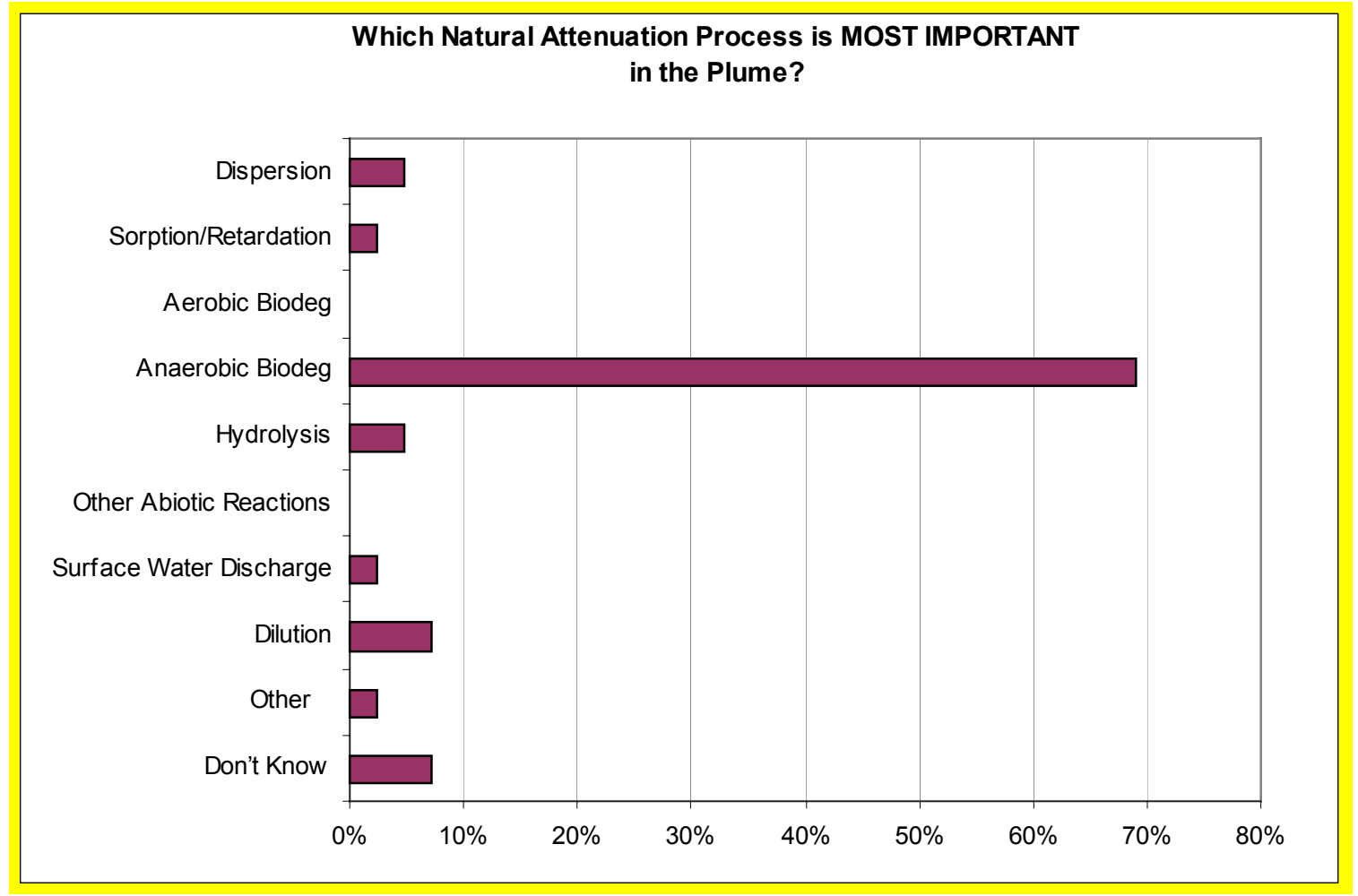

Figure 4k

How many Years of Groundwater Data were analyzed for the MNA Study?

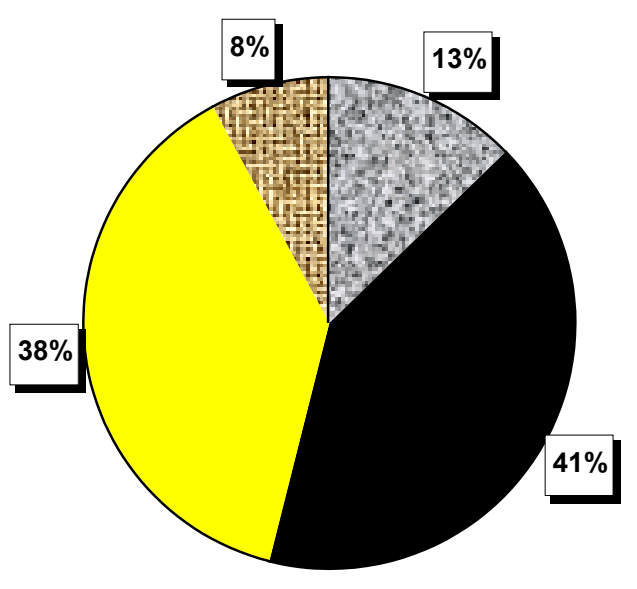

1 to $2 \square 2$ to $5 \square 5$ to $10 \quad$ 国>10 $\square$ Don't Know

Figure 41 


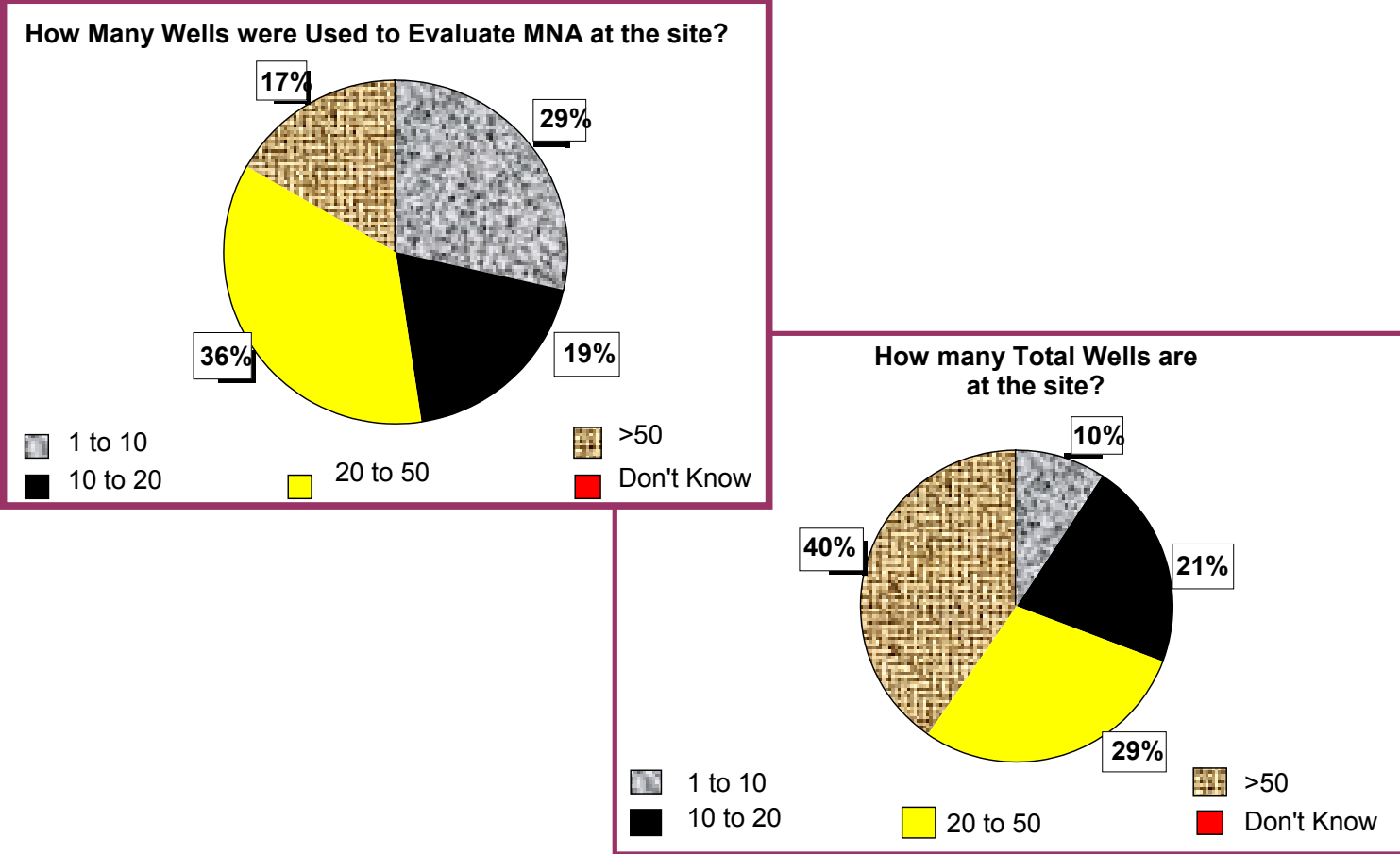

Figure $4 \mathrm{~m}$

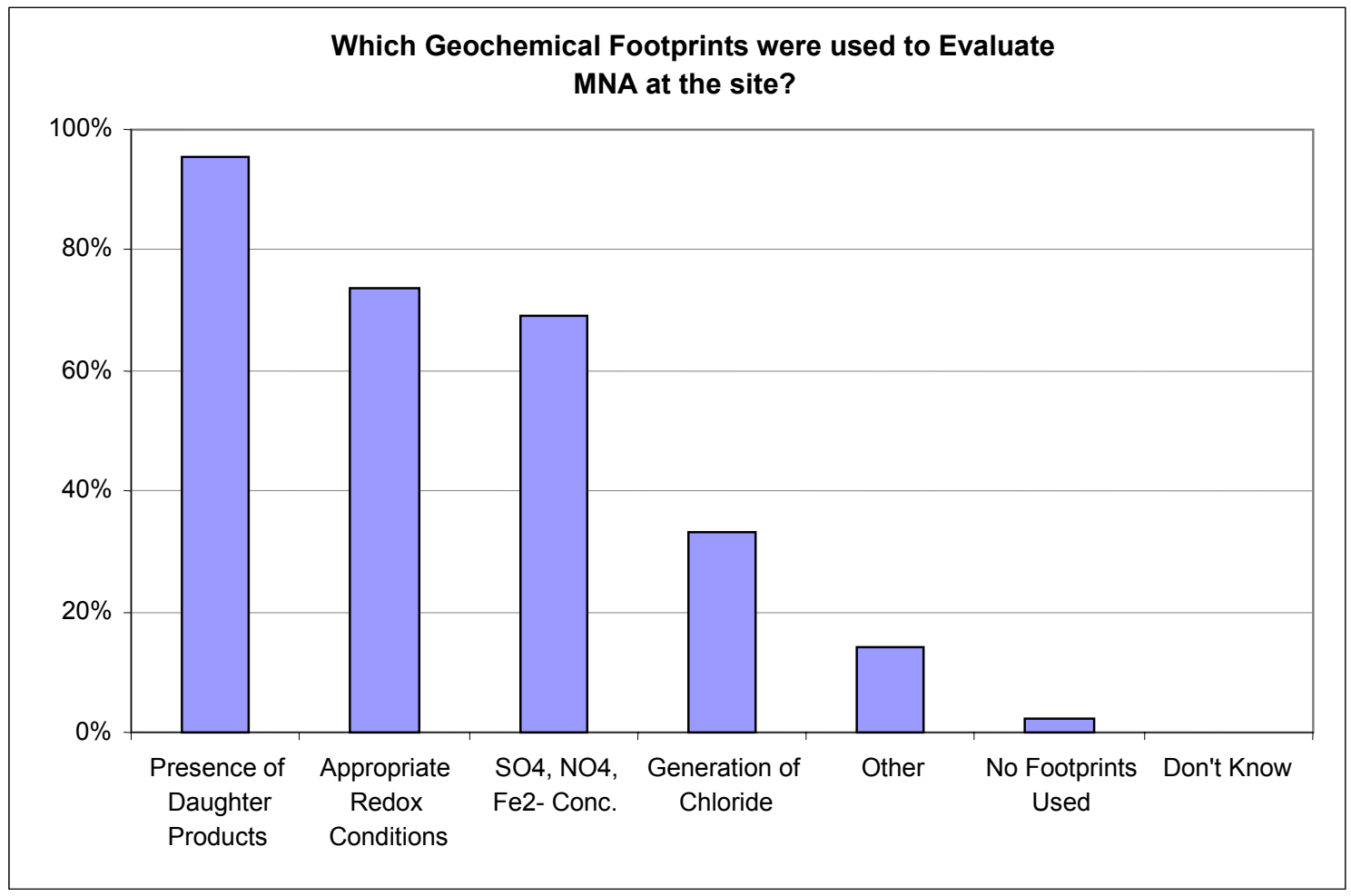

Figure 4n 


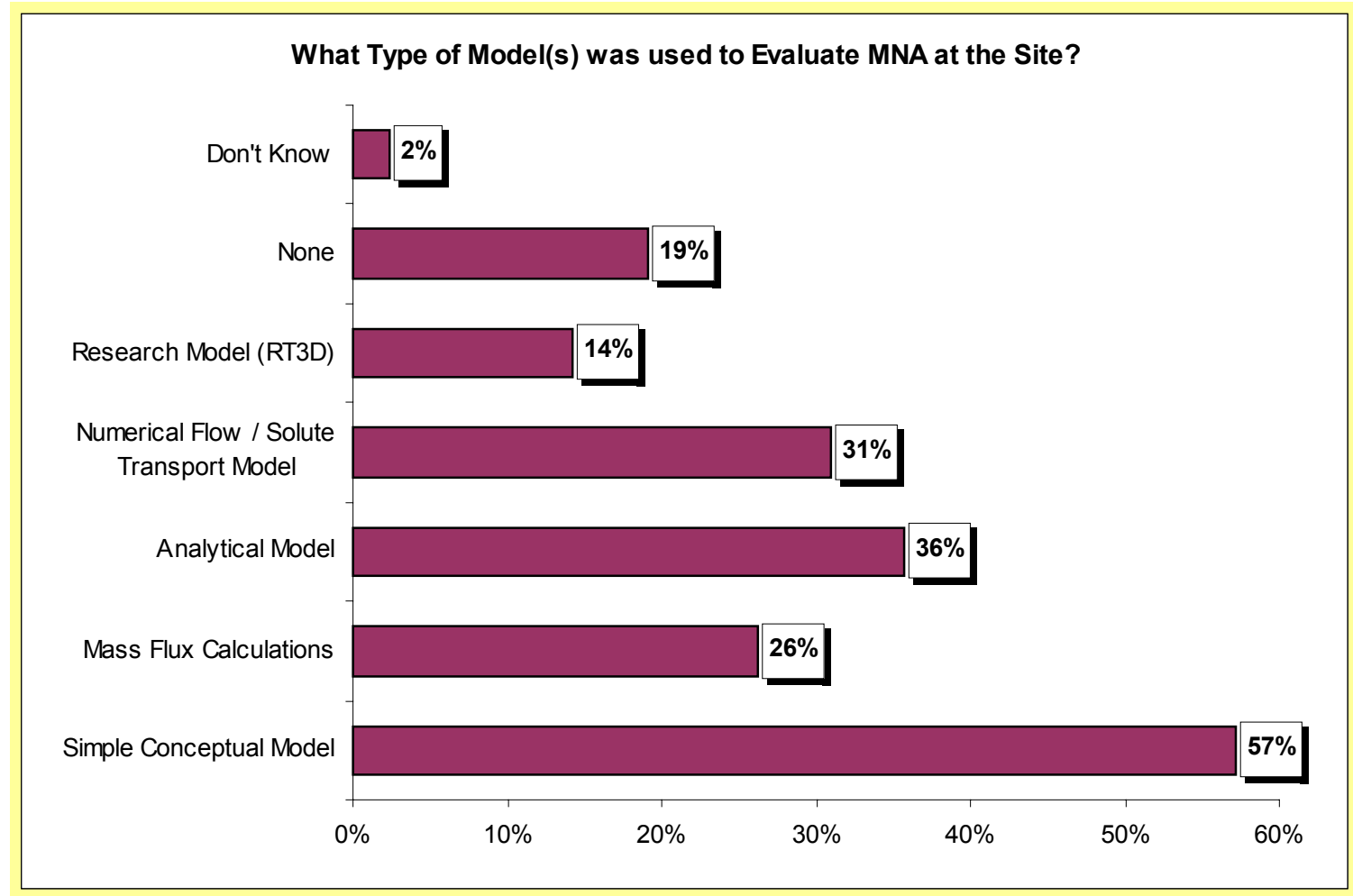

Figure 4o

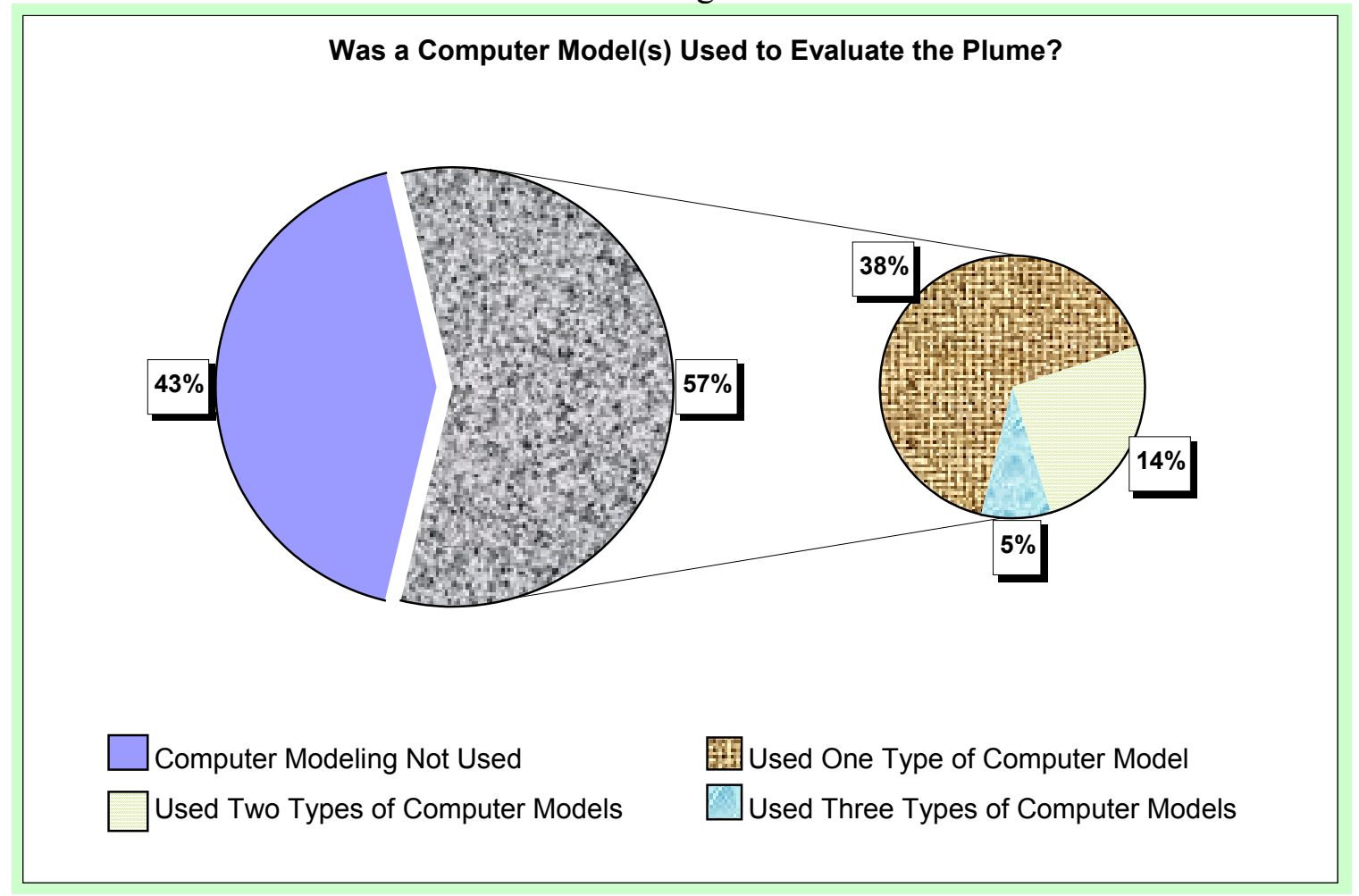

Figure $4 p$ 


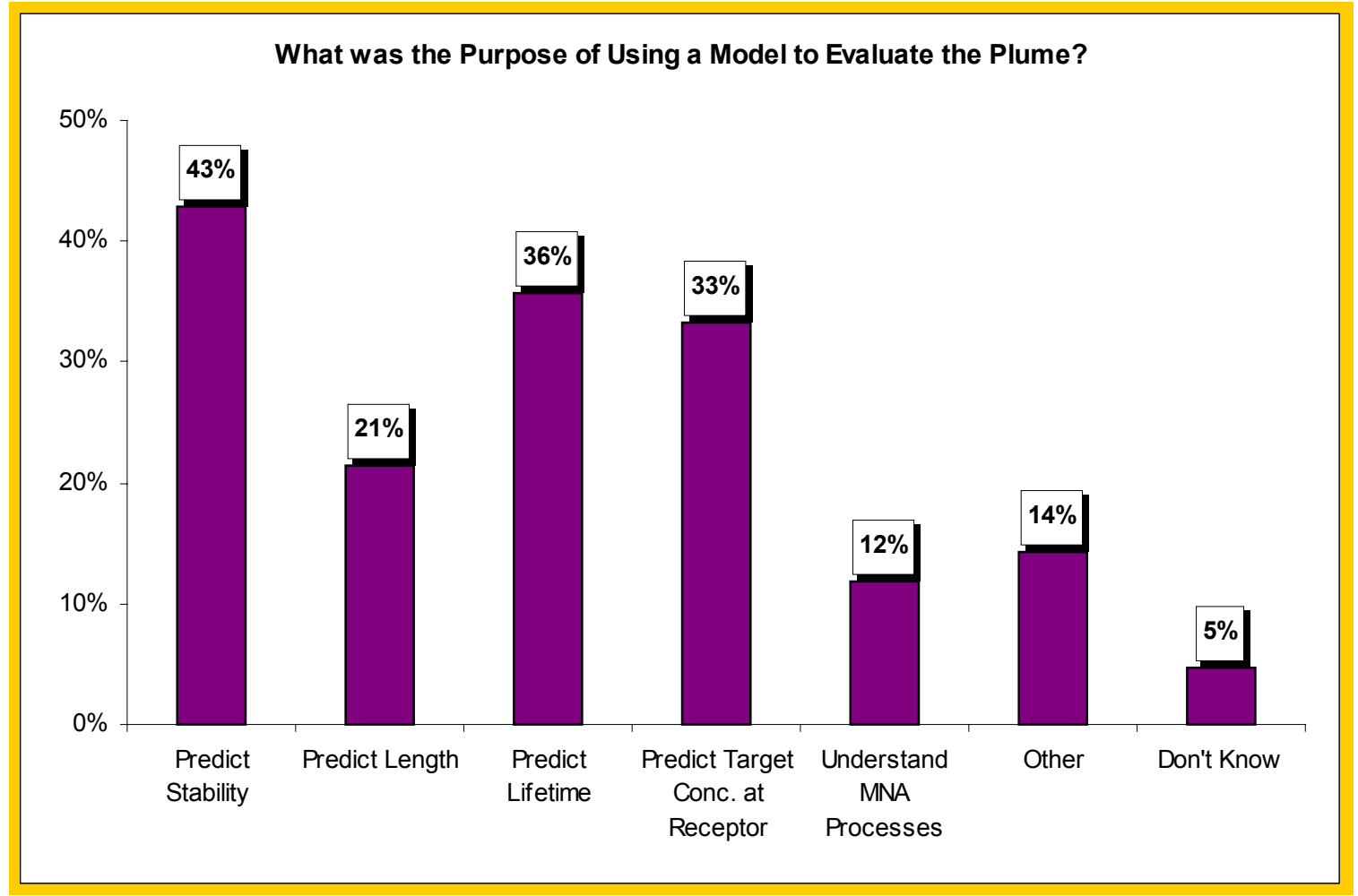

Figure 4q

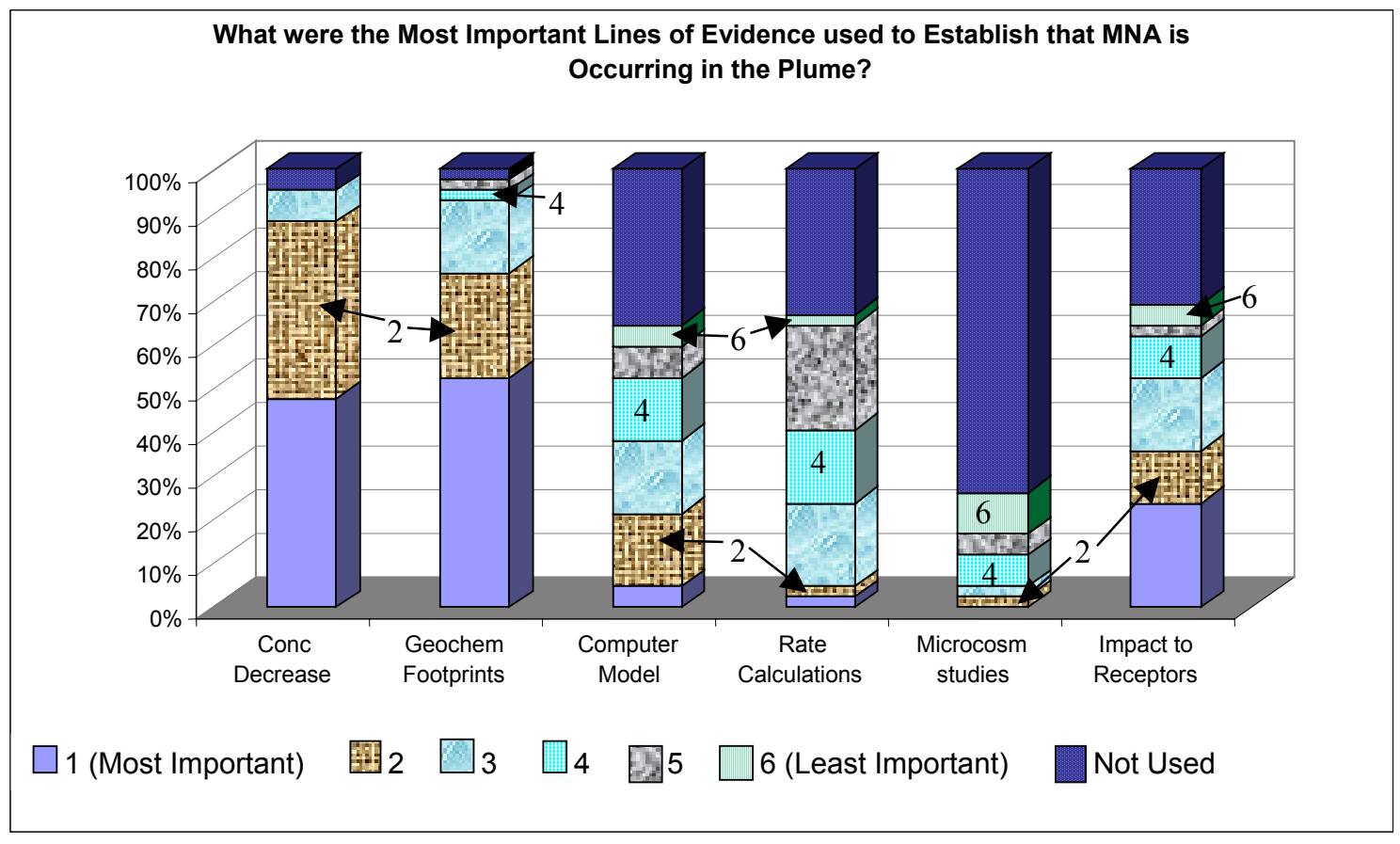

Figure $4 r$ 
Were Studies Performed to Evaluate Sustainability of Anaerobic Biodegradation within the Plume?

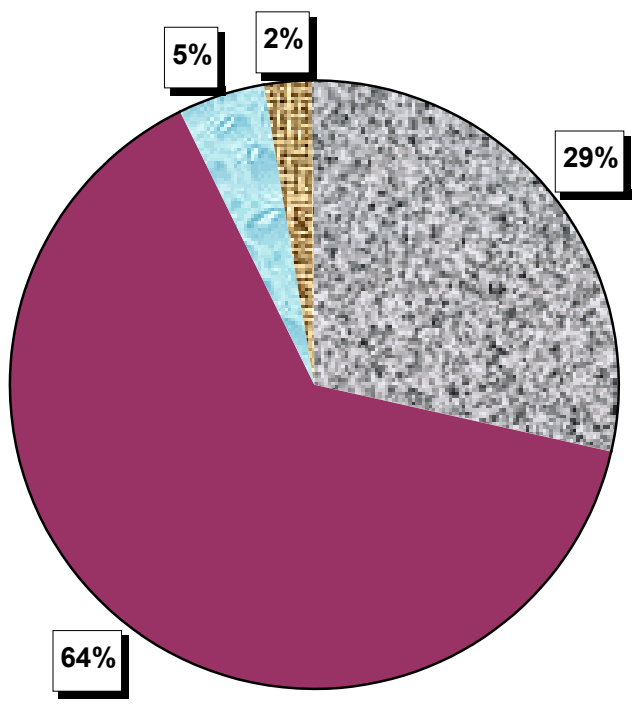

Q Yes $\square$ No $\square$ No Anaerobic Biodeg 北Don't Know

Figure 4s

Was an Analysis of Remediation

Timeframe for the Plume Performed?

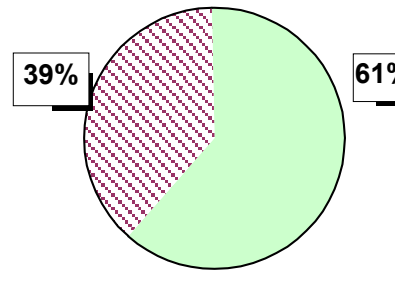

$61 \%$

Yes No

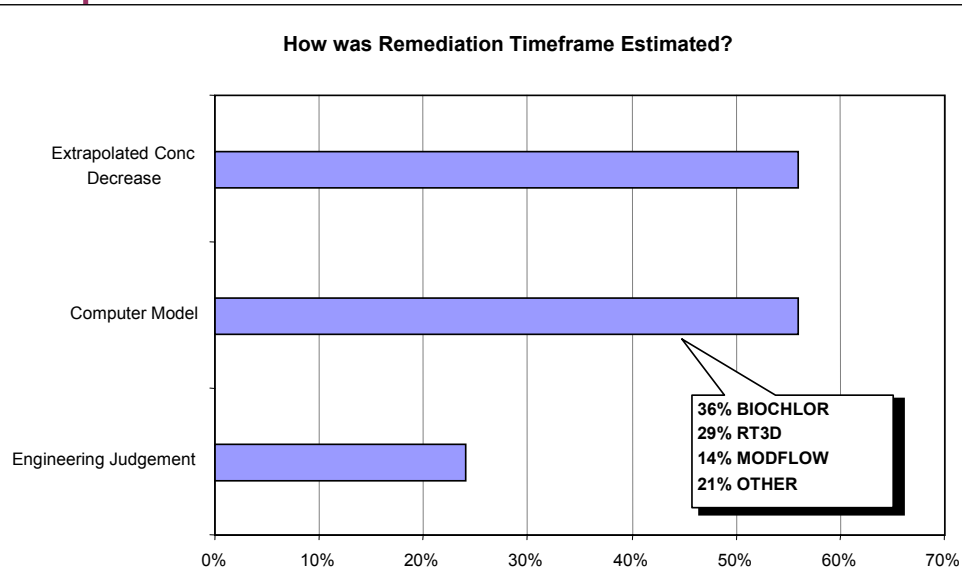

Note: $44 \%$ of Respondants who estimated Remediation Timeframe used more than one method

Figure $4 \mathrm{t}$ 


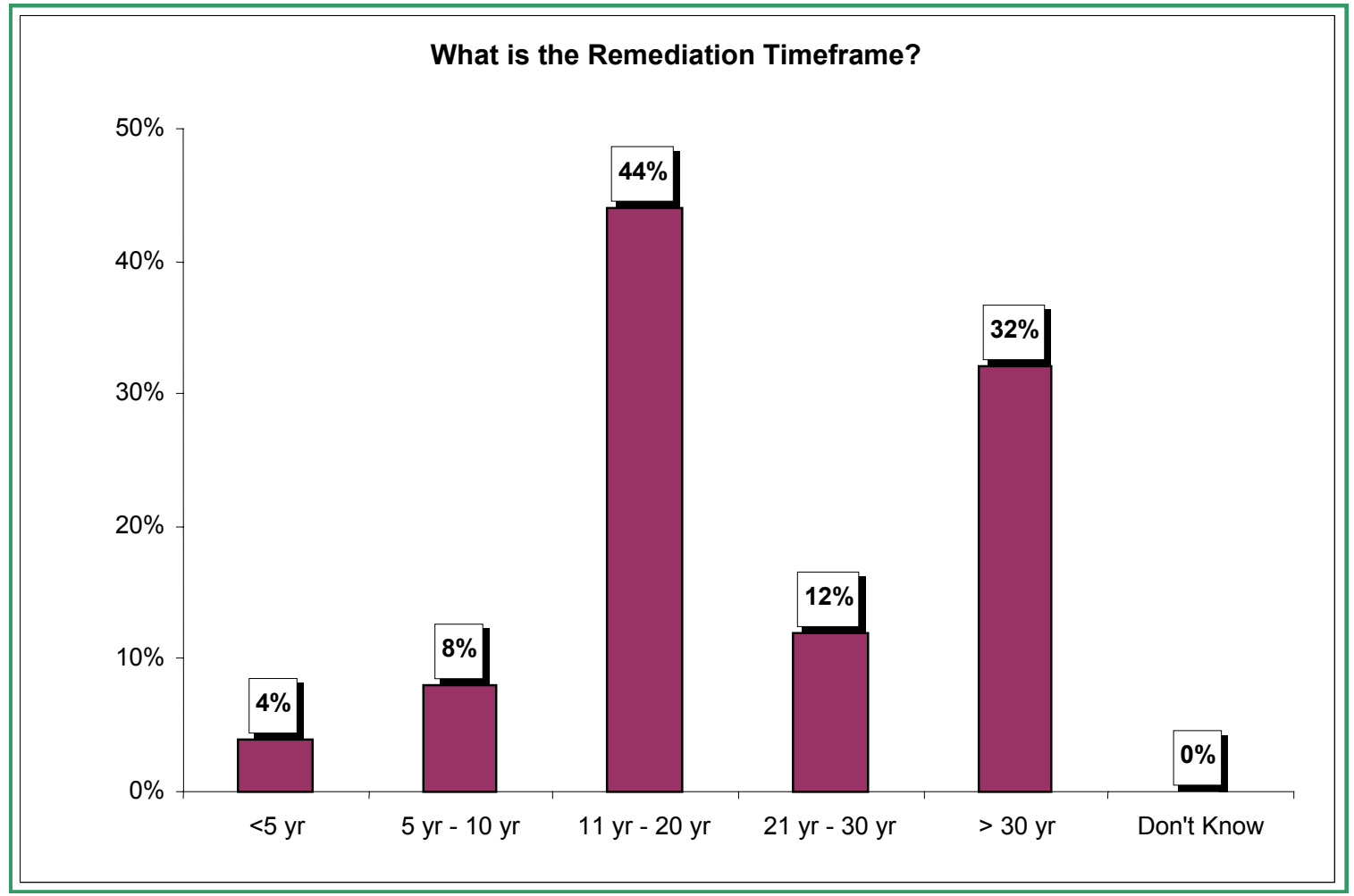

Figure $4 u$

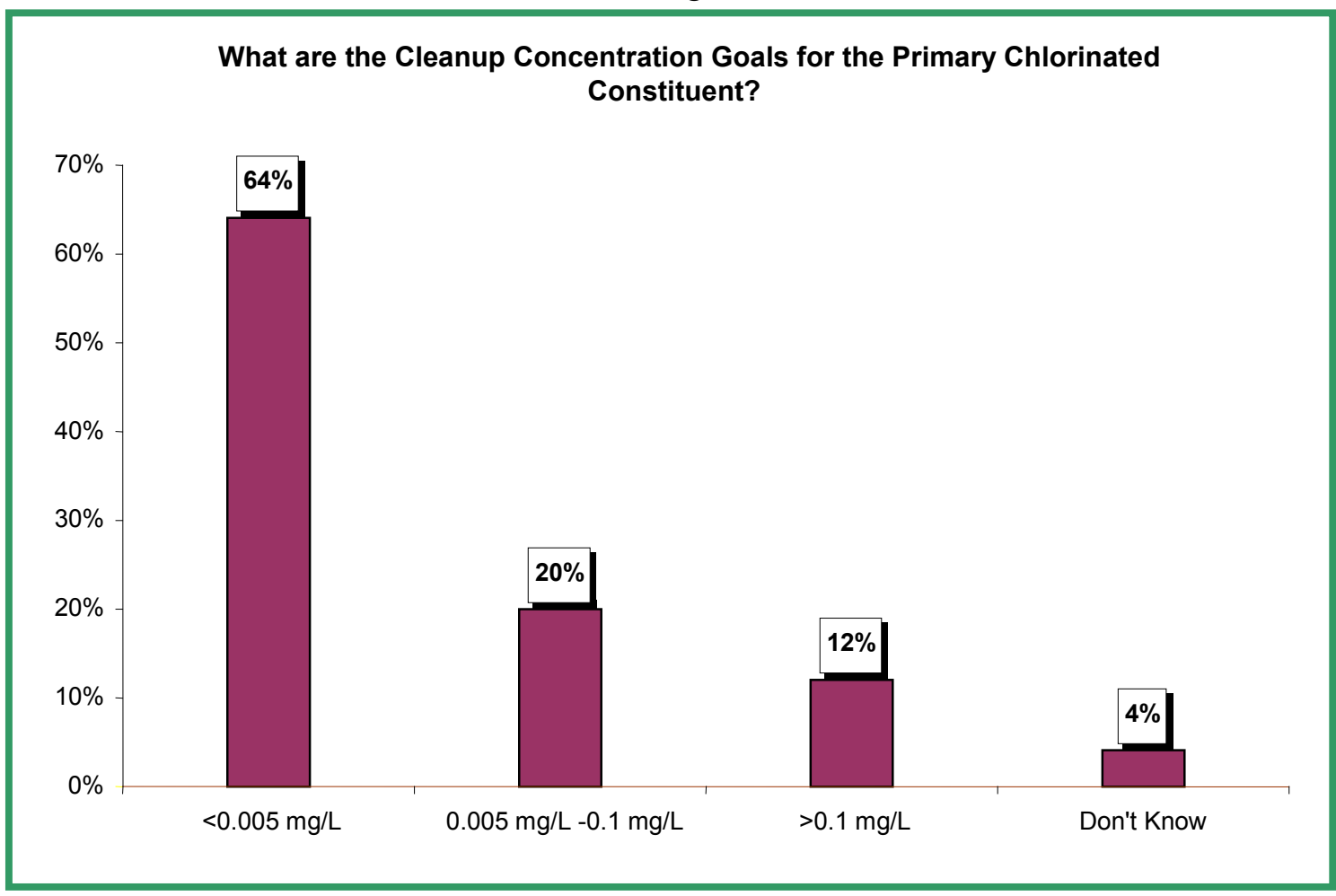

Figure 4v 
Approximately how much did the MNA Field Program and Study Cost?

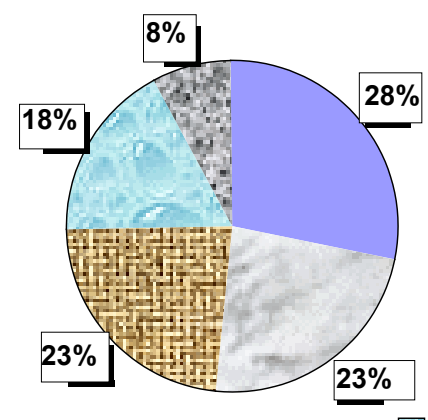

$\$ 100 K-\$ 200 K$ 国 \$50K-\$100K Don't Know

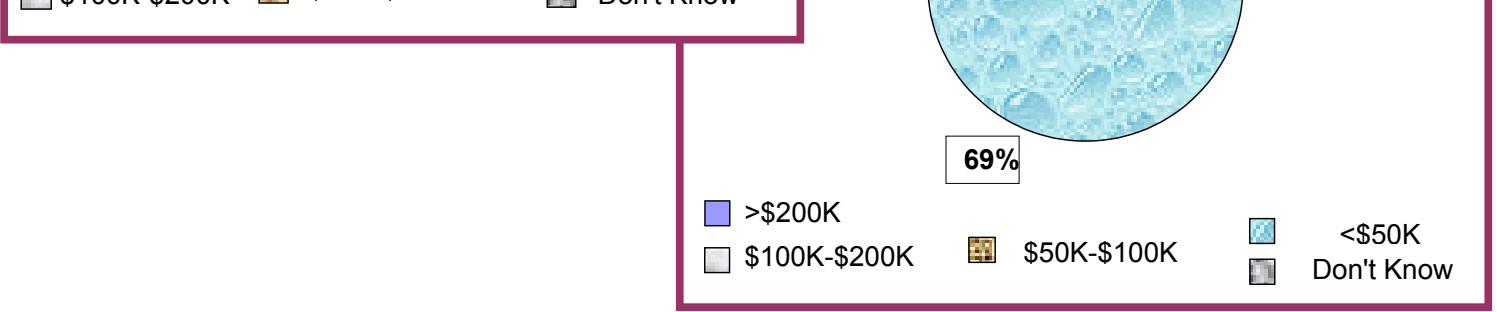

Figure 4w

What was the Original Chlorinated Compound Released at the Site?

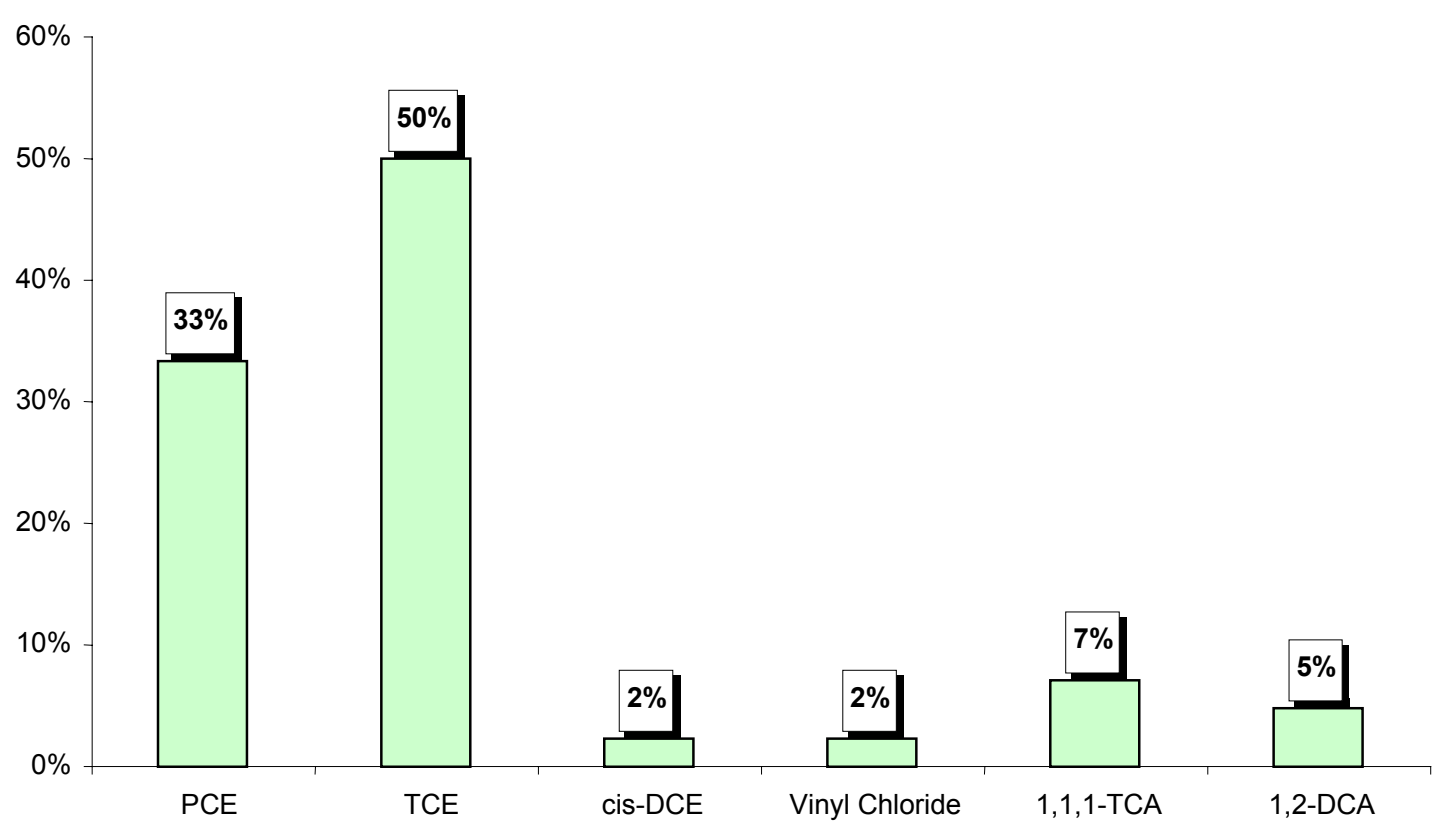

Figure $4 \mathrm{x}$ 


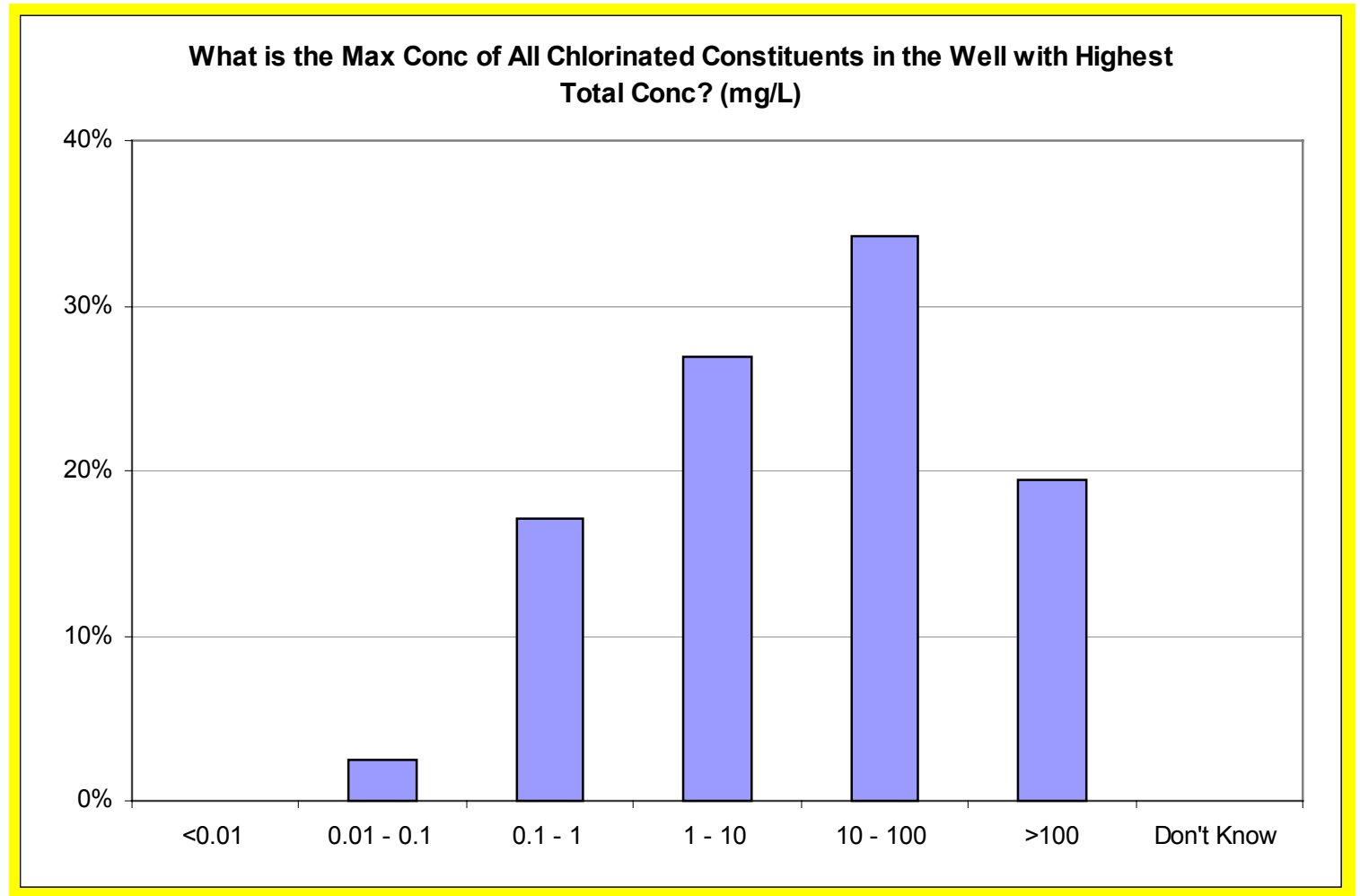

Figure 4y

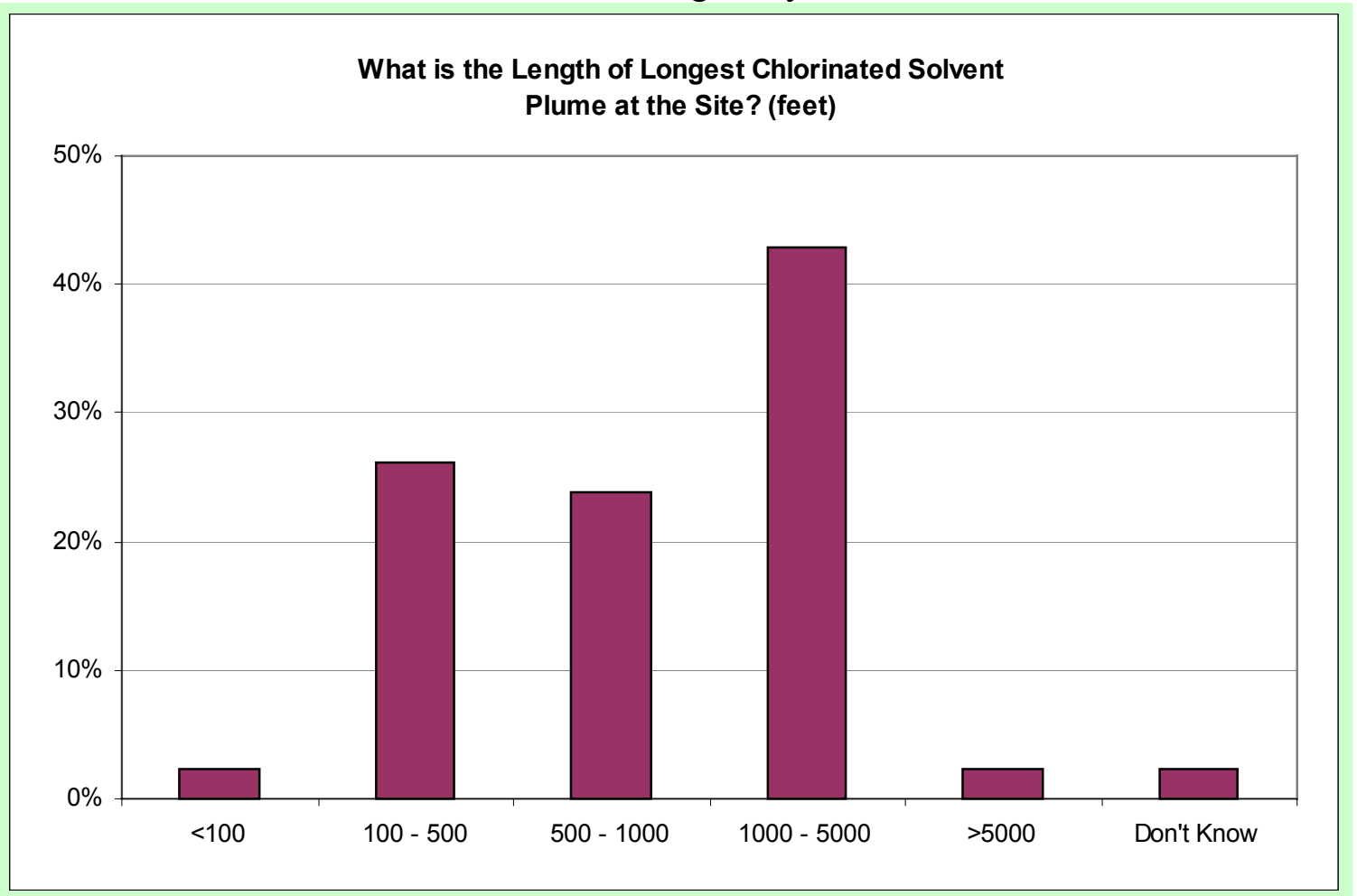

Figure $4 \mathrm{z}$ 


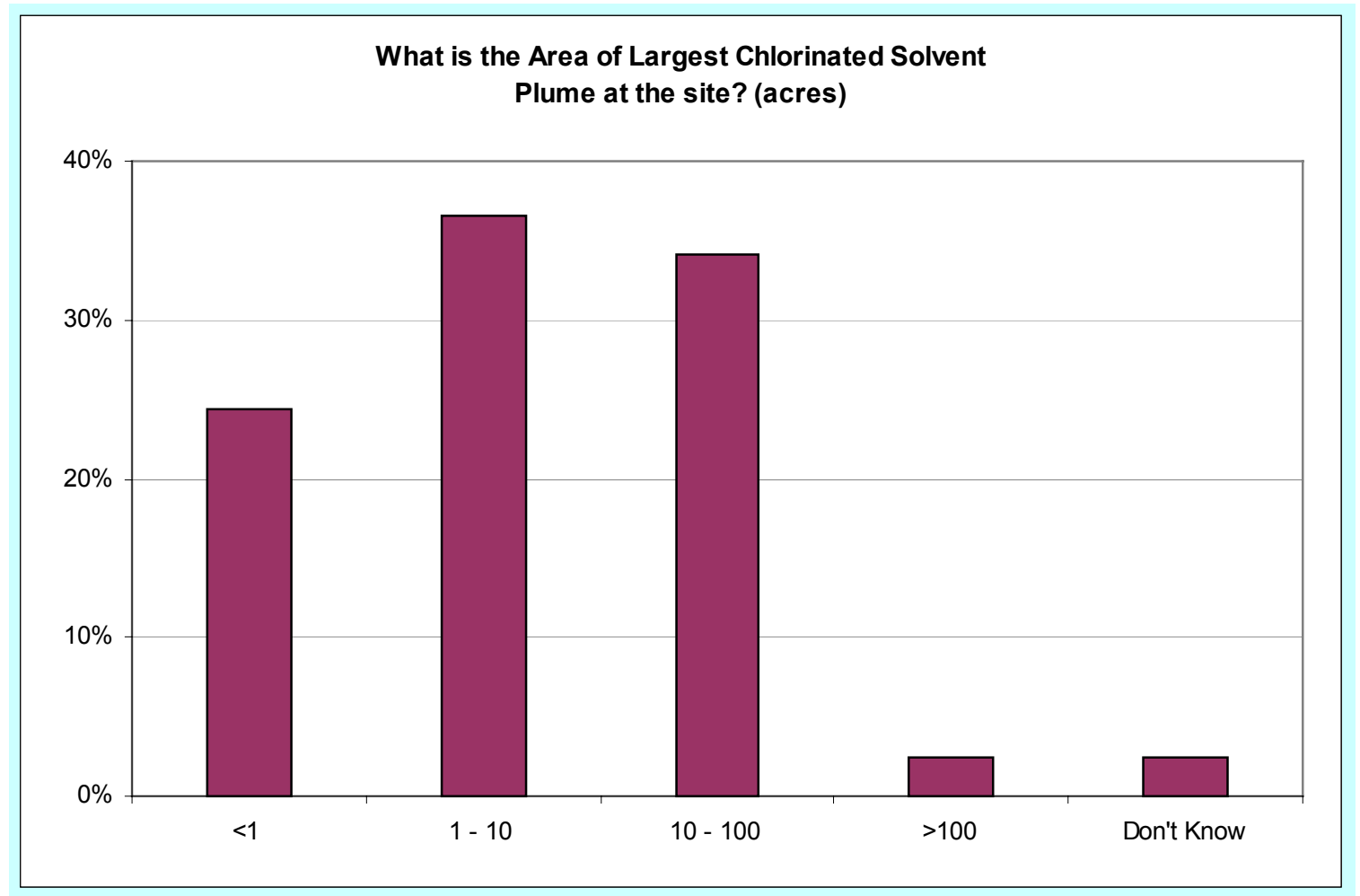

Figure 4aa

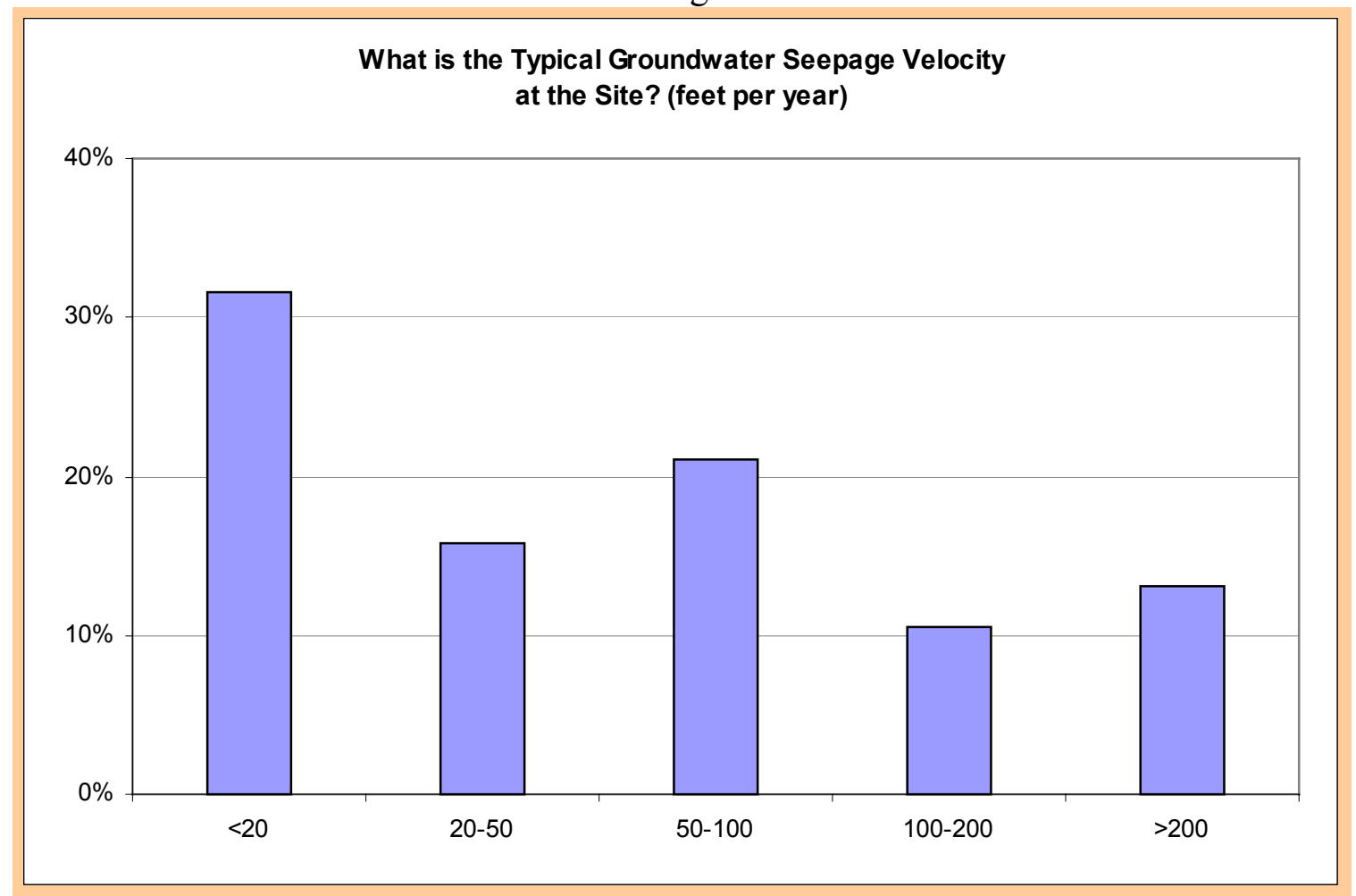

Figure 4bb 


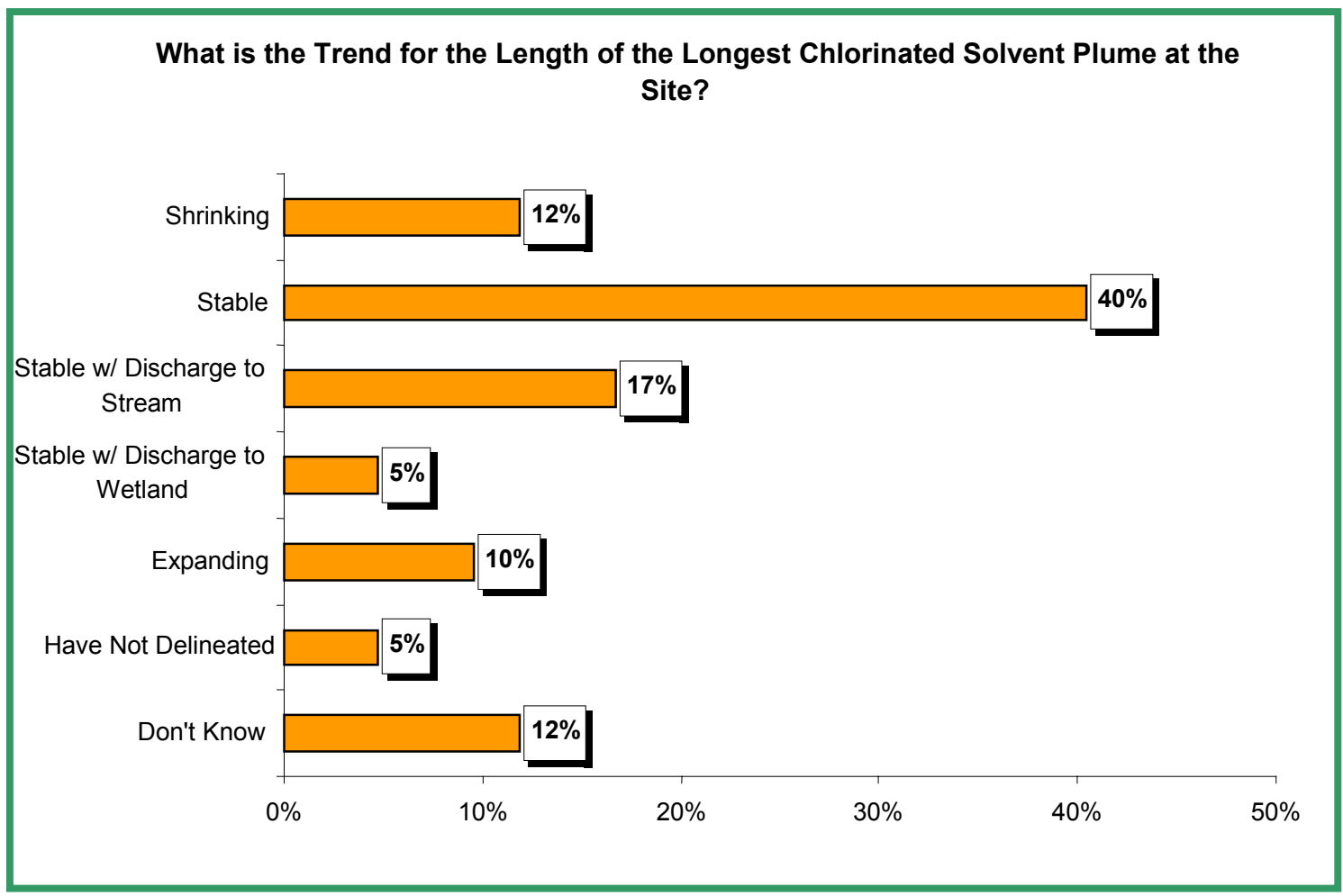

Figure 4cc

Does the Condition of cis-DCE "stall"

Appear to be a Problem at the site?

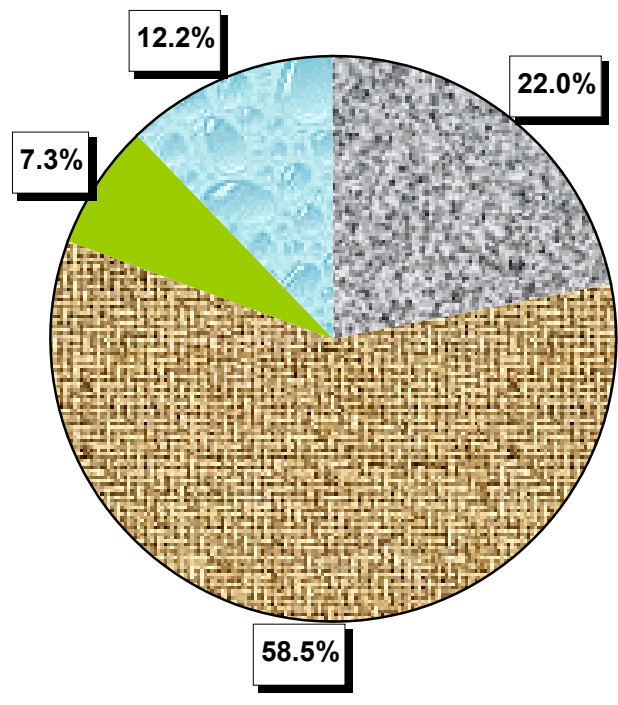

Figure 4dd 


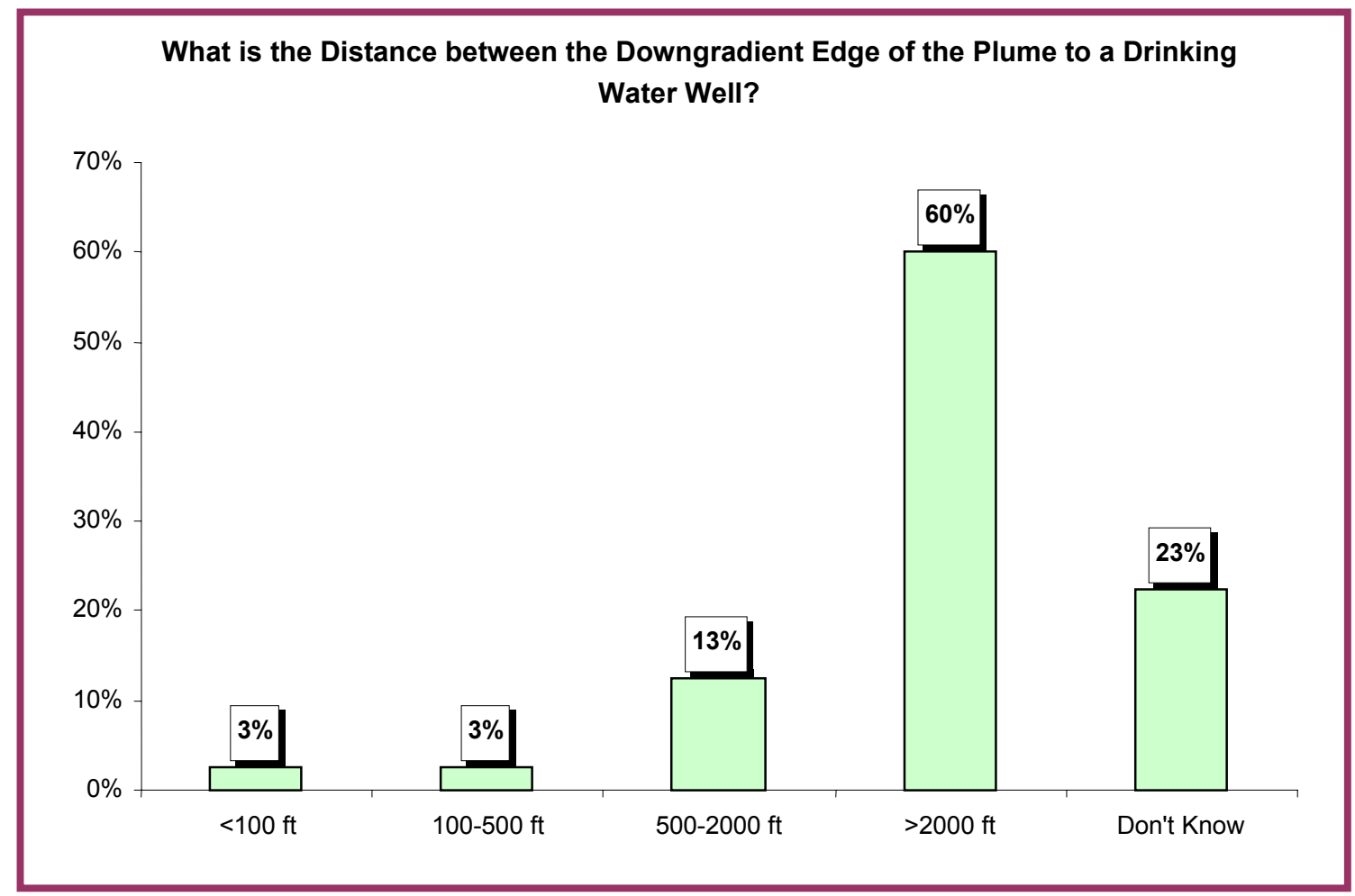

Figure 4ee

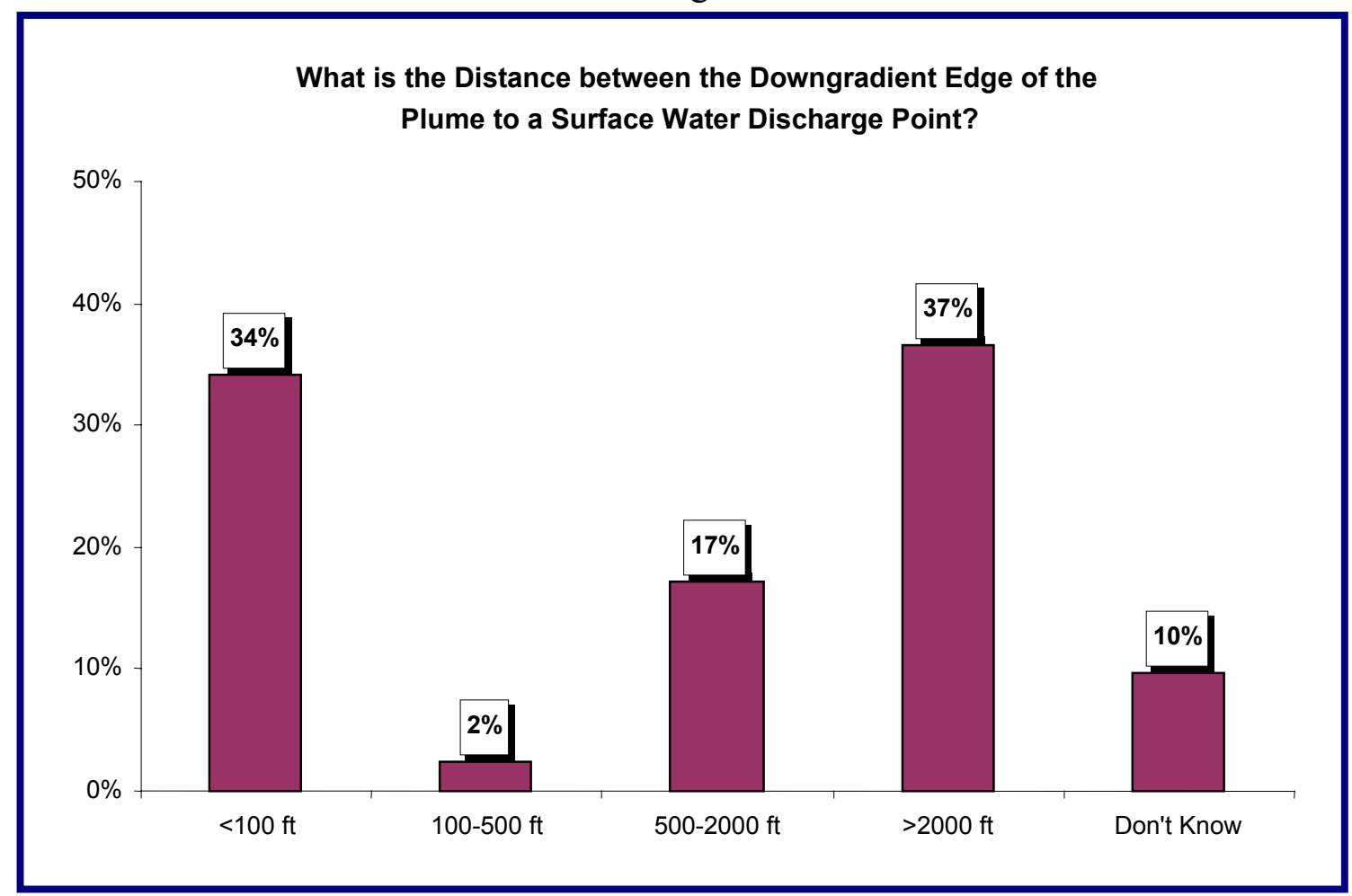

Figure 4ff 


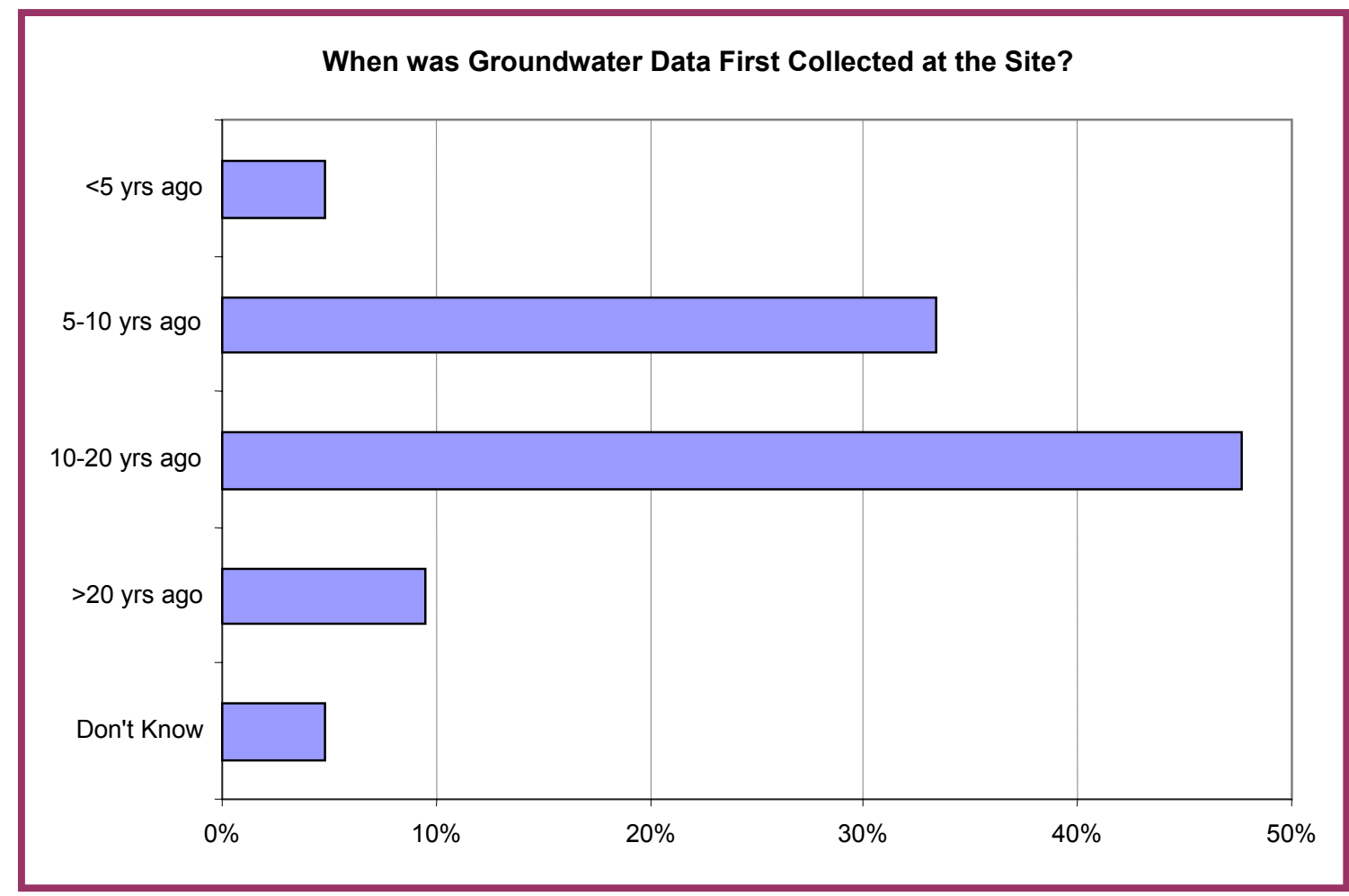

Figure 4gg

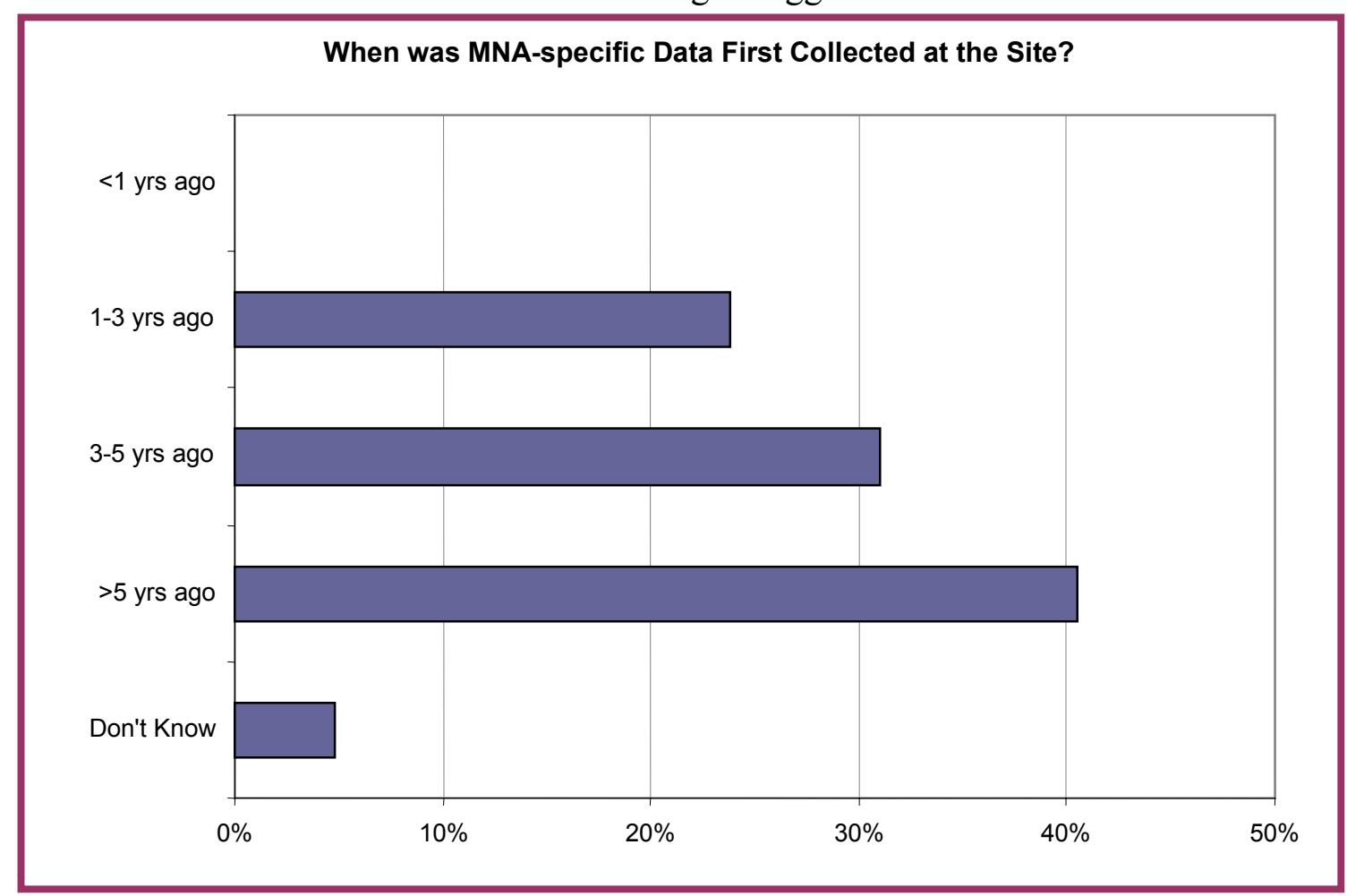

Figure 4hh 
WSRC-TR-2003-00328

February 19, 2004

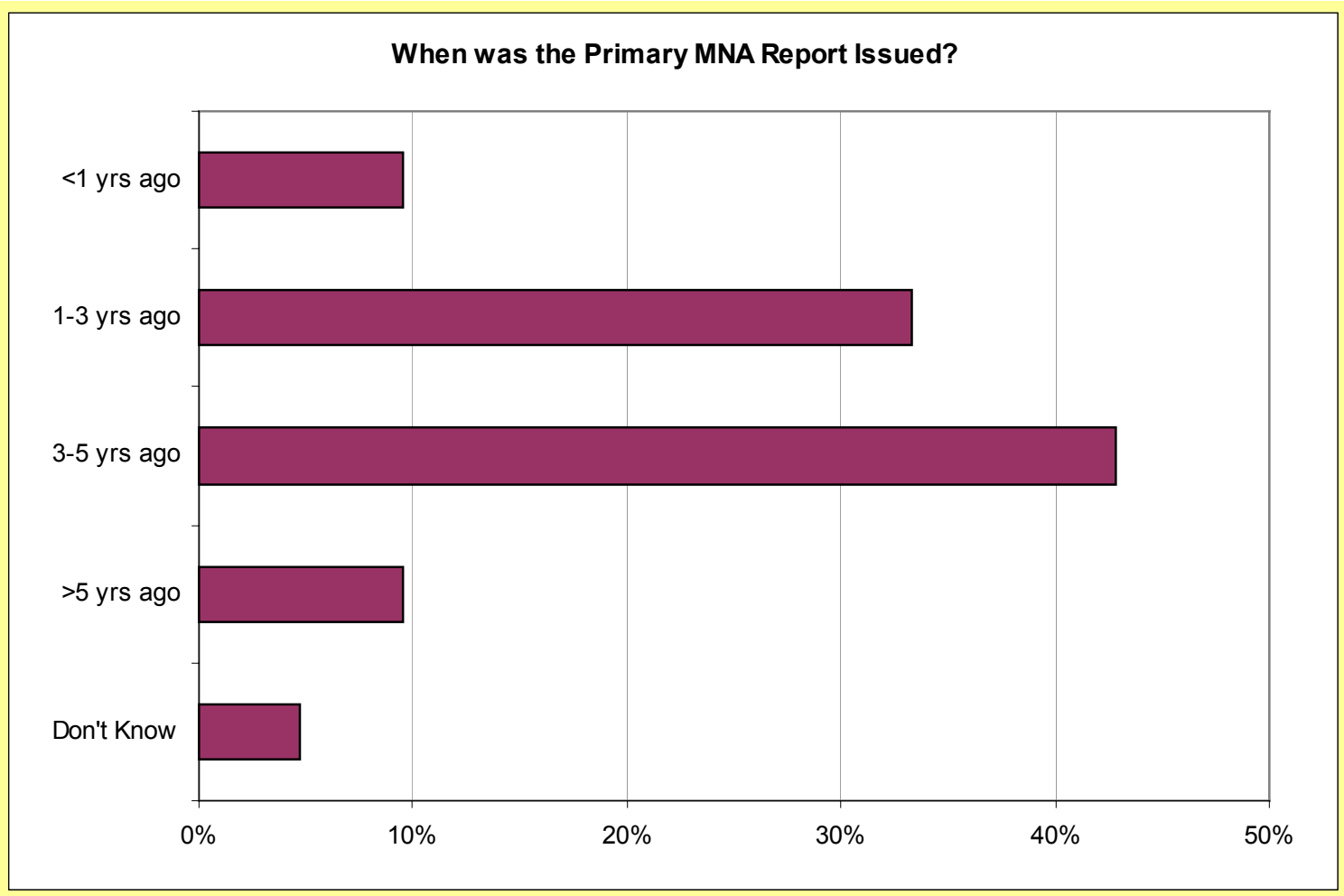

Figure 4ii

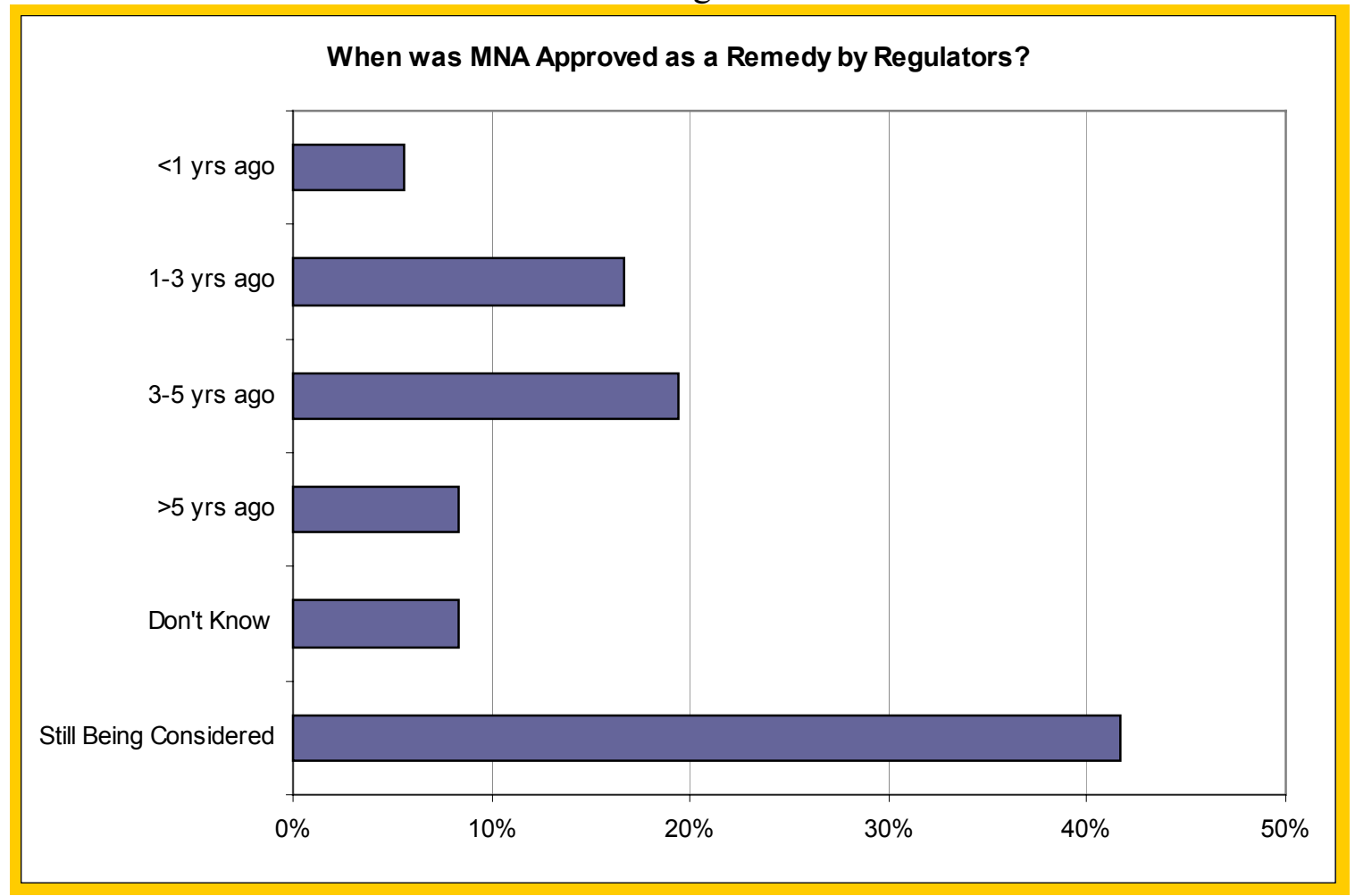

Figure 4jj 


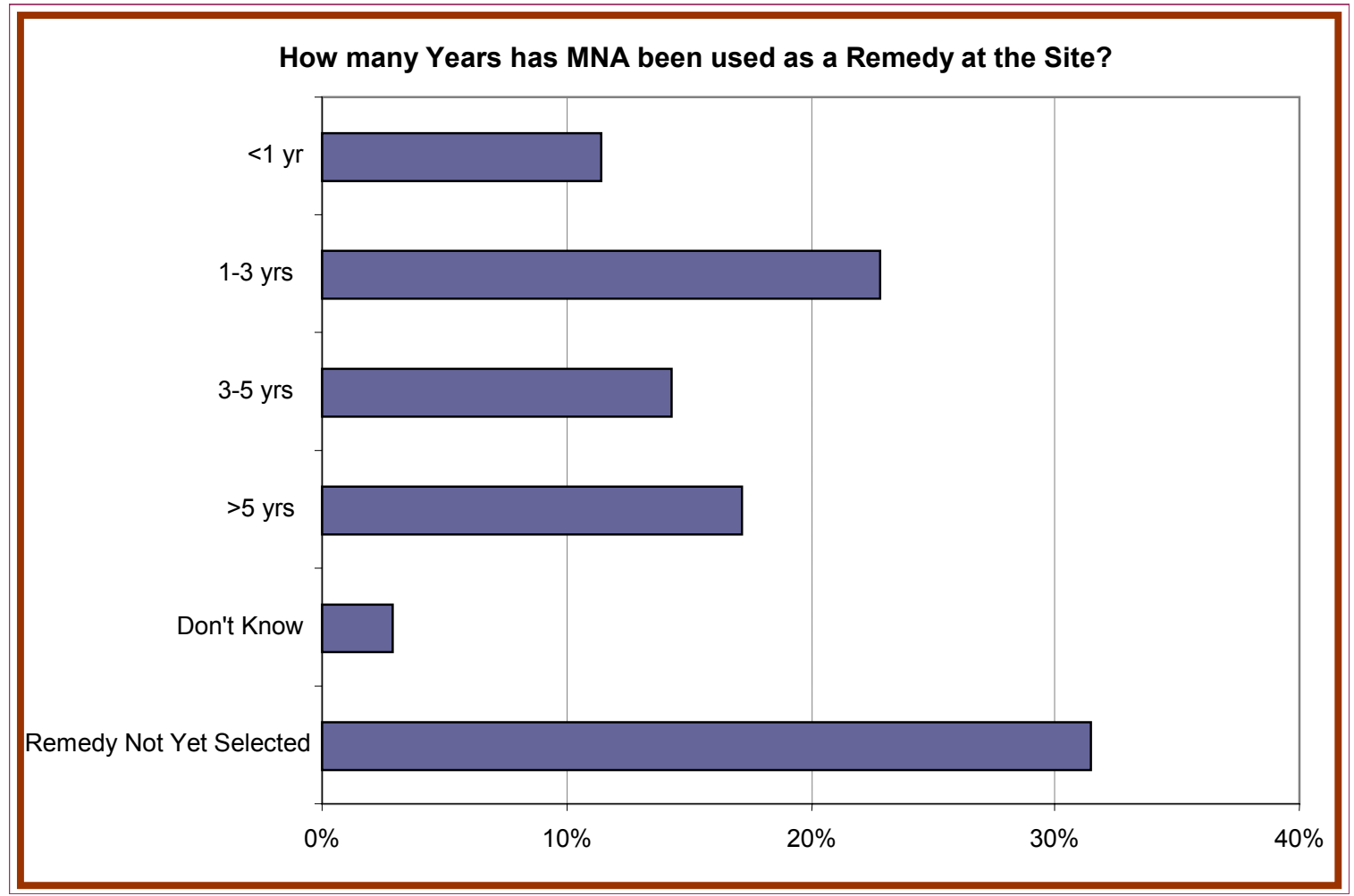

Figure 4kk

What is Your Professional Background?

Average MNA Experience $=8 \mathrm{yrs}$

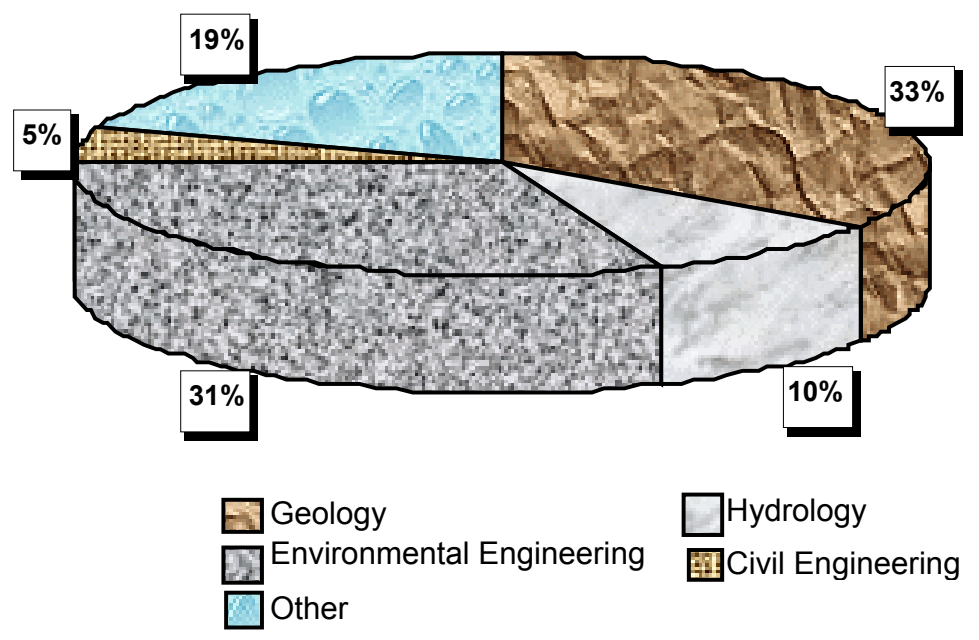

Figure 411 


\subsection{Critical Evaluation Process}

The initial activities of the national MNA/EPR Technical Working Group have focused on identification and evaluation of the specific science, technology, implementation, and regulatory concepts that have the potential to accelerate and facilitate the use of MNA/EPR and to identify those that are most promising. This evaluation was performed from March 2003 through July 2003 and the results are documented herein. This report will serve as the foundation of the US DOE sponsored MNA/EPR Alternative Project, helping direct technical decisions and the overall direction of the work, as the activities move into the field research and protocol development phases. This critical evaluation process is the primary mechanism for the project to manage costs, maintain schedule, and to assure that the product will provide the best value to US DOE.

\subsection{Lines of Inquiry}

The Technical Working Group critically evaluated past MNA/EPR protocols and performance, along with related research. This critical review process was organized into several lines of inquiry as specified below. The team documented the potential benefits of progress in each line of inquiry and the resulting improvement in the applicability and usability of MNA. For each line of inquiry, the evaluation factors included specific benefits resulting from progress (with the most credit going to items that result in a positive "step change" in MNA/EPR performance); regulatory/stakeholder acceptability; collateral and lifecycle issues; potential cost of the required science and technology; and the potential for leveraging existing science efforts. The lines of inquiry were set up in two general categories that address: 1) the scientific basis of MNA/EPR (processes) and 2) the validation and verification of MNA/EPR (characterization and monitoring).

In their evaluations, the team identified science and technology advancements that had the most potential to make a substantive contribution in a short period of time (circa three years) and those that had promise but would likely require a significantly longer period of development. Because of the objective for acceleration, the prioritization activities favored those concepts that would provide early contributions and the remainder clearly identified for consideration as promising candidates for funding in basic science programs such as the Environmental Management Science Program (EMSP). Finally, each of the team members reviewed the historical survey information as it was generated to determine if the field experience provided diagnostic information about what was working and what is needed. This was an important check and balance in the process to help avoid developing and prioritizing a solution for something that is working. 
The two general categories of the lines of inquiry are discussed below followed by a summary of each of the specific lines of inquiry. The full text of the lines of inquiry are provided in a series of supplemental documents as shown below:

Main document:

Natural and Passive Remediation of Chlorinated Solvents: Critical Evaluation of Science and Technology Targets, WSRC-TR-2003-00238

Supplemental documents:

Baseline Natural Attenuation Processes: Lines of Inquiry Supporting Monitored Natural Attenuation of Chlorinated Solvents, WSRC-TR-2003-00329

Potential Enhancements to Natural Attenuation: Lines of Inquiry Supporting Enhanced Passive Remediation of Chlorinated Solvents, WSRC-TR-2003-00330

Multiple Lines of Evidence Supporting Natural Attenuation: Lines of Inquiry Supporting Monitored Natural Attenuation and Enhanced Passive Remediation of Chlorinated Solvents, WSRC-TR-2003-00331

Potential Enhancements to the Characterization and Monitoring of Natural Attenuation: Lines of Inquiry Supporting Monitored Natural Attenuation and Enhanced Passive Remediation of Chlorinated Solvents, WSRC-TR-2003-00332

Historical and Retrospective Survey of Monitored Natural Attenuation: Lines of Inquiry Supporting Monitored Natural Attenuation and Enhanced Passive Remediation of Chlorinated Solvents, WSRC-TR-2003-00333

\subsubsection{Scientific Basis for MNA/EPR - Process Lines of Inquiry}

The process lines of inquiry align with the conceptual model, approach, and core values described in the approved US DOE Project and Implementation Plans (US DOE, 2002b; WSRC, 2003):

- Processes must be based on natural mechanisms.

- Processes must be sustainable and allow transition of the site from active treatment to a passive-monitored status.

- Approaches can include both MNA and EPR - to allow enhancements and reconfiguration (as long as the resulting mechanisms are naturally sustainable)

- Approaches should build on and link to past MNA protocols and regulatory guidance. 
- Approaches should focus on the basis for transition from active to passive to MNA/EPR and define a valid and environmentally protective exit strategy for active remediation.

- Approaches should emphasize the concept of working toward an end-state where the site poses minimal risk. The type of end-state and the risk objectives should be technically based and developed in collaboration with stakeholders, tribes and regulators.

For both the process lines of inquiry and the characterization and monitoring lines of inquiry, explicit ground rules such as these were developed to support the efforts of the team. In addition for each line of inquiry, supporting baseline discussions were prepared to simplify the evaluations. New approaches were discussed in terms of differences (pluses and minuses) versus the baseline rather than requiring each line of inquiry to separately document the baseline. The following is an outline of the process related baseline and lines of inquiry, including specific examples of some of the science and technology that was evaluated. Importantly, the outline includes a relatively comprehensive list of ideas that are being examined in current research programs, only a subset of these were determined to be promising or appropriate to incorporate into the "next generation" protocol.

- Natural Processes - Incorporation of latest research on mechanisms and rates of processes that occur without any enhancement. This includes abiotic degradation, anaerobic biodegradation, aerobic biodegradation, and phytoremediation (either in the rhizosphere or through uptake and subsequent processes). This would also include the latest research on abiotic degradation and consider sorption, dispersion and possibly instream processes such as volatilization.

- Enhancements to Natural Processes - Incorporation of latest research on processes that create permanent or semipermanent (sustainable) treatment capacity in the system. This includes:

- Microbial treatment zones - such as the possibility of halorespiring zones, substrates and conditions needed to generate and maintain such zones, addition of microorganisms, etc. This would link to the potential applicability of inexpensive characterization and monitoring of the bulk conditions (a surrogate) or microbial nucleic acids (lowcost bioassessment) as improved documentation techniques. Evaluate how far down this path is appropriate for a MNA/EPR protocol - is fertilization or other periodic maintenance acceptable with criteria to transition to monitoring only?

- Incorporation of research on deployment and enhancements based on large-scale modification of hydrology, reconfiguration of the system, 
and similar actions. One example is - expanding existing interfacial zones where treatment is occurring but total treatment is needed to fully address plume delivery (flux). Other examples include various methods for deployment of sustainable treatment zones, modification or alteration of plants (community structure and biomass, fertilization), isolation schemes (for deep fractured system for example), and schemes for sustainable large-scale alteration of bulk properties and master geochemical variables ( $\mathrm{pH}$, redox, etc.).

- Processes at System Interfaces - Incorporation of research on the active biological, chemical and physical processes occurring at major system interfaces such as the vadose groundwater interface and the groundwater surface water interface. Examine MNA/EPR potential for NAPL source zones or areas near NAPL sources (i.e., areas with aqueous concentrations close to saturation) (as is being studied extensively in US DOD).

- Examination of Scenarios - what are the needs and uncertainties associated with different conditions - notable "outcropping systems" such as those in the east, "vadose systems" as in the west, and fractured/karst systems.

\subsubsection{Characterization and Monitoring Lines of Inquiry}

The structure and concept for efforts to advance Characterization and Long-Term Monitoring are the same as those for the process lines of inquiry - i.e., 1) review of the state of practice and the state of science, and 2) critical evaluation of the potential benefits of different types of activity organized into lines of inquiry. The ground rules for these lines of inquiry are derived from the conceptual model, approach, and core values described in the approved US DOE Project and Implementation Plans (US DOE, 2002b; WSRC, 2003):

- Develop clear strategies for the distinct needs associated with MNA/EPR characterization and then monitoring.

- Emphasize integrating measures where possible, such as flux/loading, remote sensing and other averaging and volumetric methods.

- Refine the idea of "multiple lines of evidence" in current protocols and develop a defensible approach to define a "quorum of evidence" that will be acceptable (given natural variability and uncertainty). The goal is to refine and streamline characterization and monitoring, not to add more parameters to a long list of requirements for MNA/EPR.

- Emphasize large-scale design and monitoring concepts. Document performance and robustness using overall mass balances and MNA 
process/condition mapping to supplement or replace the traditional requirement of "plume stability."

- Emphasize system and ecosystem monitoring concepts.

The overarching goal of the characterization and monitoring developments was to facilitate MNA/EPR by reducing costs while maintaining or enhancing the information available to document that the system is protecting human health and the environment.

The following is an outline of the lines of inquiry and specific examples of science and technology evaluated. As noted above, the outline includes a relatively comprehensive list of ideas that are being examined in current research programs. A subset of these ideas was determined to be promising or appropriate to incorporate into the "next generation" protocol.

- Characterization and Monitoring Strategy (baseline) - examine the various stages of an MNA/EPR process and lay out the specific objectives of the stages in a manner that supports a technically based selection of the type and quantity of data needed.

- Multiple Lines of Evidence (baseline and enhancements) -Incorporate latest research and scientific logic to enhance the existing multiple lines of evidence concept. This includes a responsive characterization process based on conditional rules (i.e., is there really a need to measure reduced gases at aerobic sites). Develop a paradigm that includes some of the spatial process mapping and other items highlighted by National Academy of Sciences (NRC, 2001). Lay out a clear "quorum of evidence" concept. Incorporate the latest research on surrogate measures to reduce costs. These include bulk and master variable properties such as redox potential, as well as indicator species (e.g., $\mathrm{Cl}^{-}$) and specialized tools such as total halocarbons, degree of chlorination sensors, etc.

- Incorporate the latest bioassessment tools. These include nucleic acid probes, fatty acid profiles, taxonomy, structure and function screening profile systems, fluorescence methods, and other tools. Many of these technologies have been examined for innovative field deployment in US DOE - at ORNL, SRTC, and other labs. Other bioassessment tools include hyperaccumulators (possibly coupled with remote sensing) and macrobioaccumulators (clams, etc.), and biomarkers (ecosystem structure and species composition). These latter tools integrate exposure and may provide a more realistic measure of impact. 
- Nonstandard Monitoring Concepts-Incorporate latest research on remote sensing, geophysics, and flux monitoring. This includes both instrumentation and interpretation and deployment options (horizontal wells, lidar ${ }^{1}$, remote sensing, and others). Examine lessons from agriculture and soil science ("smart farming") and potential for cross over applicability.

- Modeling - Incorporate latest research on bioinformatics and modeling. This includes data mining, neural networks, incorporating new types of data, integrating diverse types of data, working at sites with large amounts of data and determining the value of data to justify reducing the number and frequency of analyses. Consider the latest progress in both forward and inverse predictive modeling and the potential value of large-scale mass balance models (i.e. simple balancing delivery and treatment capacity) as an alternative that might be used at many sites.

- Sensors - Incorporate the latest research on state-of-the-art sensors. Determine the value of sensors in characterization and in monitoring MNA/EPR systems. Examine the need for sensors that provide high frequency data. Examine alternative configurations that use on-off sensor signals rather than concentration signals as a way to reduce costs. Evaluate passive and cumulative sensors that would act similarly to bioconcentration.

○ Monitoring system configuration. This includes the geometry and numbers of monitoring points, focusing designed or identified diagnostic locations (weak points that would serve as indicators of performance throughout the system), and interface monitoring.

\subsection{Output from the Line of Inquiry Evaluations}

The team completed their examinations of the various lines of inquiry and the results are presented below. Summaries of the baseline evaluations are provided first followed by summaries of the enhancement lines of inquiry and the prioritization results.

"LIDAR is an acronym for "light detection and ranging." As used in the text, it refers to open path spectroscopy to estimate the total flux of VOC from the ground surface to the atmosphere over a long path length. 


\subsection{Scientific Basis for MNA and EPR}

\subsection{Summary of Natural Attenuation Processes--Without Enhancements}

The natural attenuation capacity of ground-water systems may be thought of as the capacity of an aquifer to lower contaminant mass as groundwater flows downgradient away from a contaminant source area. This capacity is the sum of all the physical, biological, and chemical processes serving to disperse, biodegrade, chemically transform, immobilize, or sequester contaminants in a ground-water system. These processes consist of physical processes such as dispersion, advection, and volatilization, chemical processes such as sorption and abiotic transformations, and biologic processes such as biodegradation and uptake by plants.

Advection refers to the migration of solutes due to the flow of water. The effect of advection is directly proportional to the velocity of groundwater flow. However, because of the heterogeneity of hydraulic conductivity in ground-water systems, the velocity of groundwater is also highly variable. The traditional method for accounting for this variability is the concept of hydrodynamic dispersion. Hydrodynamic dispersion is the sum of dispersion due to molecular diffusion and the mixing of water due to variations in groundwater velocity in the system. Because the effects of hydrodynamic dispersion are additive as the plume migrates, it is highly scale dependent with long plumes exhibiting more dispersion then short plumes. Disperson was cited in the historical survey as an active process in documenting MNA at over 70 percent of the MNA sites, but was considered the primary mechanism in the overall natural attenuation capacity in only about 5 percent of the sites.

Volatilization can be an important mechanism removing contaminant mass from groundwater systems. Chlorinated ethenes, particularly cis-DCE and VC, are highly volatile, and can be removed from contaminant plumes as they migrate downgradient. In general, volatilization is more important in contaminant source areas and in ground-water discharge areas where groundwater comes in close contact with the unsaturated zone.

Sorption is a class of chemical processes that serve to partition solutes between the aqueous and solid phases in aquifers. Thus, sorption can serve either to remove solutes from solution (sorption), or to add solute to solution (desorption). When sorption/desorption processes are relatively fast and are equally efficient, they are referred to as reversible sorption. Reversible sorption temporarily sequesters contaminants on the solid phase, and tends to retard the rate that contaminant plumes develop over time. When desorption is less efficient than sorption, and mass is sequestered in the solid phase and resistant to release, this is referred to as "irreversible sorption" or time-variable sorption. Timevariable sorption is an important component of natural attenuation capacity only if it removes contaminants from solution for an extended period of time and provides attenuation in excess of linear sorption. Sorption and retardation were cited as active 
processes in 65 percent of the MNA sites surveyed. However, sorption was considered as a minor mechanism in the overall natural attenuation capacity at most MNA sites, such that, it represented a predominant process in less than 5 percent of the sites. In some cases, sorption may be more of a hindrence than a help to MNA because the slow release of contaminant can extend the timeframe needed to reach agreed target concentrations.

A variety of abiotic chemical degradation processes can contribute to the natural attenuation capacity of ground-water systems. The most common of these include hydrolysis, dehydrohalogenation, and reductive elimination. Hydrolysis of chlorinated methanes and ethanes have been well documented, and the half-lives of these compounds can be on the order of days. Chlorinated ethenes, however, are relatively resistant to dehydrohalogenation. It has been shown that abiotic dechlorination of PCE and TCE can be catalyzed by the mineral surfaces of pyrite and magnetite. In addition, iron sulfides (FeS) will catalyze the abiotic transformation of chlorinated ethenes as well. Hydrolysis was an active NAC process in about 8 percent of the sites surveyed and the other abiotic reactions also were active in 8 percent. However, abiotic reactions, with the exception of hydrolysis, were not considered important NAC processes until very recently and thus have not been investigated on a large scale. Based on recent literature, further investigation is likely to indicate that abiotic processes may be important for the many sites that are at least sulfate reducing. In the historical survey, hydrolysis was considered as a primary process in about 5 percent of the sites.

Biodegradation processes are an important component of the natural attenuation capacity of chlorinated ethenes. The mechanisms by which chlorinated ethenes are transformed depend on whether they serve as electron donors or electron acceptors in microbial metabolism. In reductive dechlorination, chlorinated ethenes serve as electron acceptors. In oxidative degradation, chlorinated ethenes serve as electron donors. The more highly chlorinated an ethene is (i.e. PCE), the greater its tendency to undergo reduction and the less its tendency to oxidize. Conversely, the lightly chlorinated ethenes (VC) have less tendencies to reduce and more tendencies to oxidize. Intermediately chlorinated compounds, such as cis-DCE, can be degraded either reductively or oxidatively. However, because cis-DCE is neither highly oxidized nor highly reduced, it tends to be more recalcitrant in ground-water systems than other chlorinated ethenes. Because of the redoxdependent nature of chlorinated ethane biodegradation processes, understanding the distribution of redox processes in ground-water systems is an important part of assessing biodegradation processes. Often, it is the sequence of redox processes that determines the overall contribution of biodegradation to natural attenuation capacity. For example, if strongly reducing methanogenic or sulfate-reducing conditions are dominant in a contaminant source area, these conditions favor the reduction of PCE and TCE to cis-DCE and VC. As groundwater flows downgradient, and redox conditions grade to more oxidizing, Fe(III)-reducing, and even oxic conditions, cis-DCE and VC will rapidly oxidize. Hence, this succession of strongly reducing to oxidizing conditions favors the complete biodegradation of chlorinated ethenes. Conversely, the opposite succession of 
redox conditions (oxic to methanogenic) is less favorable for the complete biodegradation of chlorinated ethenes.

There have been significant advances in recent years in identifying the actual microorganisms involved in chlorinated ethene biodegradation. Dehalococcoides ethenogenes strain 195 has been shown to completely dechlorinate cis-DCE to ethene. Other isolates such as Desulfitobacterium, Dehalobacter restrictus, Desulfuromonas, and Dehalospirillium multivorans have been shown to dechlorinate one or more chlorinated ethenes. There have been several reports that the extent of dechlorination is proportional to the presence or absence of Dehalococcoides sp., a finding which may allow the efficiency of reductive dechlorination to be accessed by quantifying the abundance of these microorganisms. Molecular methods for quantifying the presence of dechlorinating bacteria are potentially powerful tools for evaluating the contribution of reductive dechlorination to natural attenuation capacity. However, the phylogeny of microorganisms involved in oxidative biodegradation of chlorinated ethenes has not advanced as rapidly as those involved with reductive dechlorination. This reflects the difficulty of isolating environmental strains of bacteria in pure culture where their physiology can be characterized. It is likely that only a small minority of the microbial species capable of biodegrading chlorinated ethenes is presently known.

Anaerobic biodegradation was cited in the historical survey as an active NAC process in over 90 percent of the sites and the most important NAC process in approximately 70 percent of the sites. Aerobic biodegradation was not as active as anaerobic or physical processes at most of the sites, being listed as an active NAC process in about 30 percent of the sites. Aerobic biodegradation was not considered an important NAC process at any of the MNA sites. Importantly, anaerobic degradation is relatively effective for highly chlorinated solvents (such as PCE and TCE) while aerobic processes are effective for less chlorinated solvents (e.g., DCE and vinyl chloride). As a result, the optimum attenuation condition can vary from site to site, and from one part of a site to another. The data suggest that the presence of oxygen (or by analogy any other strong oxidant or alternate electron donor such as nitrate, perchlorate or sulfate) will strongly inhibit anaerobic degradation while lack of an alternate electron donor will strongly inhibit aerobic oxidation. Some sites have dissolved oxygen present and many sites are co-contaminated with electron donors with a resulting influence on MNA/EPR strategy and viability.

Another biological process that can be significant to the natural attenuation capacity of ground-water systems is uptake by plants. Certain plants can remove, transfer, stabilize, and destroy chlorinated ethenes in soils, sediments, groundwater, and surface water. A prerequisite for plant uptake of contaminants is that they be non-toxic to plants. Research has shown that plants have the ability to withstand relatively high concentrations of organic contaminant concentrations without interfering substantially with plant metabolism. This, in turn, indicates that plant uptake of chlorinated ethenes---what has been termed phytoremediation---can contribute substantially to the natural attenuation capacity of ground-water systems. A number of processes including rhizodegradation, rhizofiltration, 
phytodegradation, phytovolatilization and photodegradation, phytoextraction, and phytostabilization (including hydraulic containment) contribute to this overall capacity. An important factor in the efficiency of these processes is the access the plants have to contaminated water. For this reason, phytoremediation tends to be most efficient in areas with shallow contamination such as groundwater discharge areas where plant roots have direct access to the saturated zone.

\subsection{Enhanced Passive Remediation Opportunities}

The physical, chemical, and biological components of the natural attenuation capacity have an impact on the magnitude of the flux of contaminants reaching a receptor. However, these processes may not be sufficiently robust to reduce the flux to within acceptable limits in undisturbed systems. Therefore, this section considers what potential actions might be feasible that will enhance their effectiveness by stimulating a natural process to be more active, work faster (improved kinetics), proceed to a more favorable end-state, or to improve the overall attenuation of contaminants beyond what is occurring naturally. The fundamental question is, "Can we either manipulate the system (e.g. hydrology) and/or the processes to achieve a desired end-state?"

There are several major objectives of any proposed enhancements. Enhancements must a) increase attenuation of the contaminant (and/or decrease the flux of contaminants from the source that is feeding the plume) and b) once implemented, be sustainable (within acceptable limits). In this line of inquiry we consider a range of enhancements to the following general processes:

- Microbiological degradation

- Abiotic processes

- Plant-based processes

- Large-scale hydrologic processes

- Modifications at interfaces

Data indicates that some of these processes do not work efficiently enough in some natural systems to provide sufficient attenuation within a plume to meet regulatory requirements. For example, under certain circumstances native bacteria provide incomplete degradation of the solvents such that toxic intermediate constituents such as DCE or VC are formed, but not destroyed at appreciable rates. Are there ways to stimulate the existing consortia (or add new, more favorable bacteria) to more completely degrade contaminants? Will such an enhancement, once implemented, be sustainable with little or no further intervention?

With respect to plants (herbaceous and woody) there are several dimensions to the possibility of enhancements. For example, can one take steps to improve the ability of the native plants to uptake and transpire or metabolize the contaminant? Can one enhance the biochemical processes naturally taking place in the root zone of native plants to increase the in situ destruction of the contaminants? Alternatively, one can consider introducing a 
new suite of plants that have been selected to have special properties for removing the contaminant either due to enhanced uptake/metabolism (e.g. hyperaccumulators) or improved/refined root-based reactions. Finally, careful manipulation of the type of trees introduced, their density of growth, and their specific placement relative to the contaminant plume can manipulate the amount of uptake and transpiration of groundwater and thereby modify the groundwater flow system or the amount of infiltration reaching the water table. The purpose of this enhancement is to manipulate the groundwater flow system in desirable ways or reduce the flux of contaminants reaching the aquifer from a contaminated source zone.

Large-scale hydrologic modifications are designed to either fully or partially isolate the source of the plume or modify the flow system throughout the plume footprint by simple engineered methods in order to reduce the mass flux of contaminants within limits that can be accommodated by other natural attenuation processes. These can include various forms of surface caps and covers as well as subsurface engineered barriers that reduce the flux of contaminants by altering groundwater flow patterns.

The question of sustainability of enhancements is fundamental to the EPR approach. There is no specific timing for the frequency of intervention that is explicitly acceptable or unacceptable. Biennial application of fertilizer to sustain favorable plant growth may not be too intrusive within the context of cost, level of effort, and results attained. In contrast, replacement of engineering enhancements (e.g. caps or covers) on a decadal time-scale may be too frequent. Therefore, the question of sustainability must be considered on a case-by-case basis.

The following sections will develop potential lines of inquiry that may lead to strategies for enhancing the effectiveness of natural attenuation processes. Figure 5 identifies these lines of inquiry at a high level. Detailed discussion of them is contained in the supplemental document, Potential Enhancements to Natural Attenuation: Lines of Inquiry Supporting Enhanced Passive Remediation of Chlorinated Solvents, WSRC-TR-2003-00330.

In principle, enhancements to these natural processes can be implemented throughout the entire contaminated system (source, plume, and discharge region). However, the value of such a spatially encompassing strategy probably is limited because the potential effectiveness of any enhancement is not uniform, but frequently confined to relatively small regions. Selectively applying enhancements to those localized regions where the greatest impact can be achieved for the lowest cost is most appropriate. 


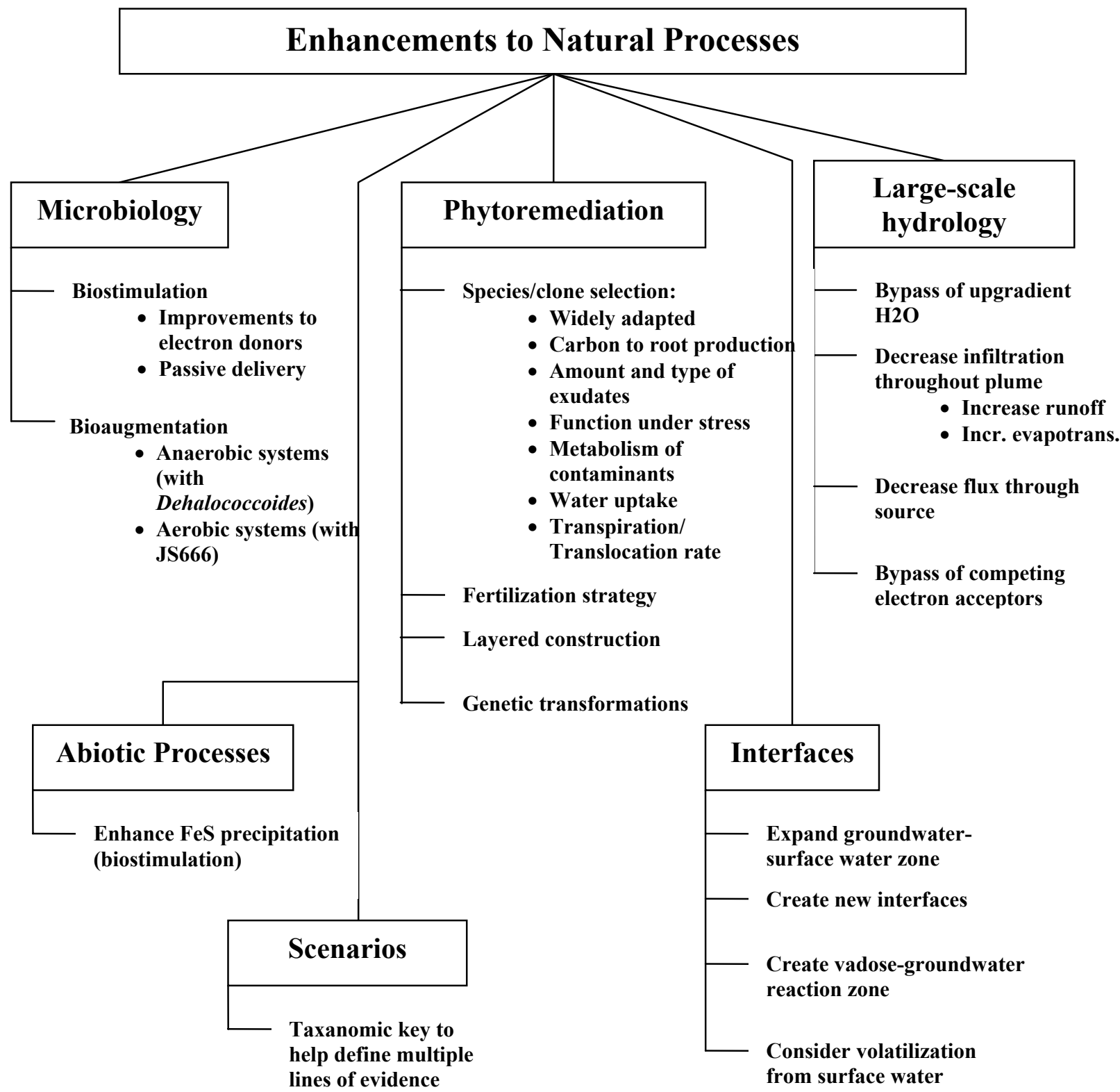

Figure 5 Lines of inquiry for the principal components of natural attenuation where enhancements may improve their efficiency.

For example, using engineered or phyto-based approaches in the source region has the potential to reduce the flow of groundwater or infiltration of recharge water through the source zone. These modifications directly impact the contaminant flux or mass loading to a 
plume. Likewise, in the vicinity of the discharge zone, conditions are favorable for microbial contaminant degradation and phytoremediation. Enhancements that improve the efficiency of these processes can be localized to greater benefit. In contrast, for the bulk of the contaminant plume, access is more difficult and costly and the scale of the plume itself may make application of enhancements less desirable in this region. Figure 6 is an illustration of the concept of spatial distribution for implementation of enhancements.

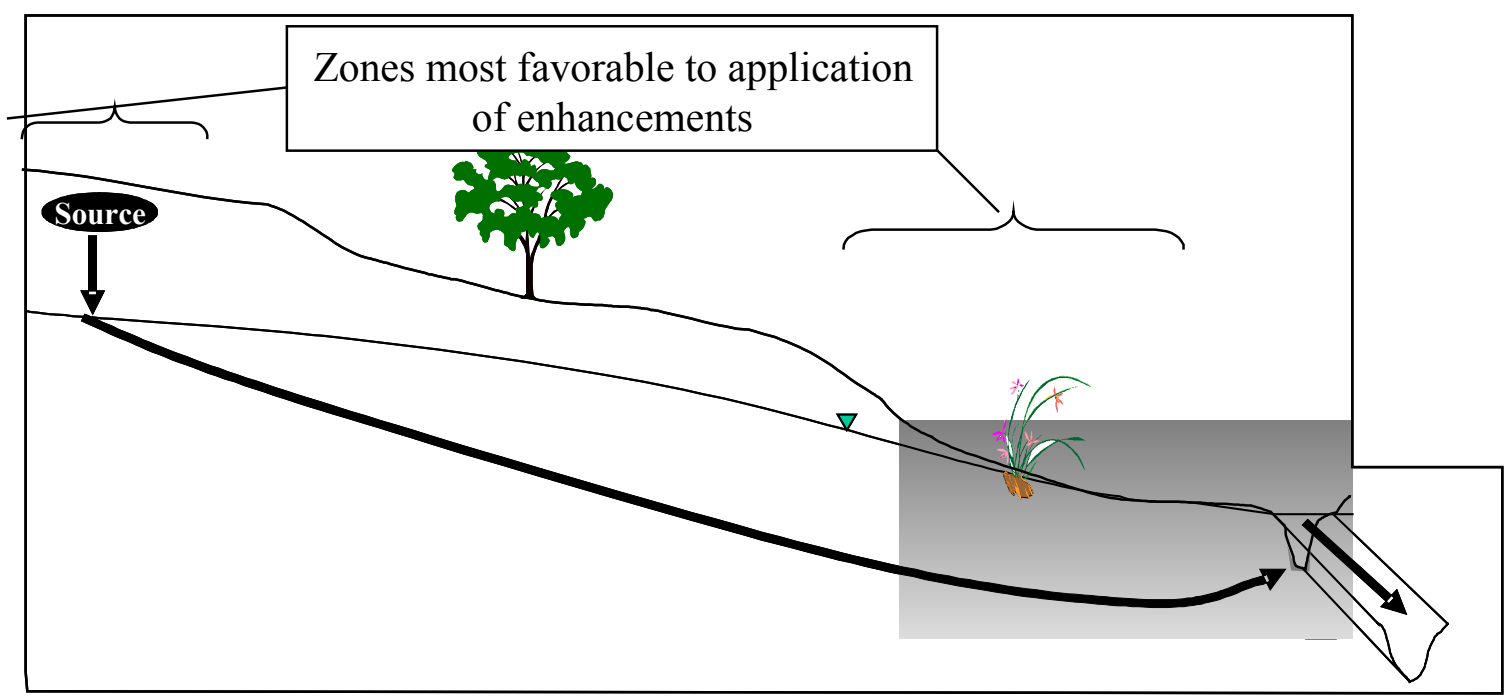

Figure 6 Locations within a watershed most favorable for introducing enhancements to natural processes.

\subsubsection{Microbiology}

Biostimulation is a technical approach that utilizes the addition of electron donor or electron acceptor at a contaminated site to enhance in situ microbial activity. The primary goal of biostimulation is to provide reactants that are currently limiting a targeted in situ microbial activity. In some cases, biostimulation may be used to alter fundamental in situ conditions (e.g. $\mathrm{pH}$, redox, sulfate/nitrate removal) to facilitate a desired remediation strategy. A prerequisite to the successful use of biostimulation is the development of a clear technical strategy developed from a scientific understanding of microbiology, geology, hydrogeology, groundwater chemistry, etc. Biostimulation is typically utilized at sites which have a latent in situ biopotential that is currently under-utilized due to limitation of one or more key components.

Biostimulation for chlorinated solvent remediation most commonly utilizes the addition of electron donors at sites that are carbon-limited. The addition of exogenous organic substrate results in stimulation of in situ microbial activities and enhanced capacity for biodegradation of chlorinated solvents. In recent years, there 
have been numerous reports that in situ microbial degradation of chlorinated solvents was successfully stimulated by the addition of a variety of carbon sources. Typically, these reported projects have utilized continuous or repeated injection of organic substrate. The most widespread active biostimulation successes have used proprietary reagents such as Hydrogen Release Compound (HRC from Regenesis Inc), lactate (many practicioners), or sugars/molassas (e.g., ARCADIS and others). More recently, there has been growing interest in the use of longer-lived materials such as vegetable oils or extended release HRC for biostimulation. As discussed below, this latter group of compounds may be useful to help move toward EPR from active biostimulation.

A key technical opportunity area for biostimulation is the need for improved, cost-effective sources of electron donors designed to enable the sustained release of organic substrates over long timeframes. This need could be met with improved slow-dissolution materials designed for strategic placement into a contaminated system (e.g., bark mulch and peat based treatments). Alternatively, an opportunity also exists to engineer passive delivery systems that reliably deliver nutrients over longer time frames. In both cases, the benefits of this proposed technology development is to enable biostimulation to be conducted in a more passive manner resulting in reduced operating and maintenance costs. In addition, improved passive biostimulation will also indirectly benefit bioaugmentation since bioaugmentation often requires biostimulation to produce and maintain acceptable in situ conditions to support the augmented microbial processes.

Bioaugmentation is the addition of a prepared culture of microorganisms with desired degradative properties to a contaminated medium to exploit the degradative qualities to enhance contaminant biotransformation. The cultures can be either pure or mixed microbial strains consisting of exogenous or indigenous microorganisms. While the concept of bioaugmentation is not new, the historic aggressive promotion of various bacterial preparations for the remediation of non-chlorinated hydrocarbons that failed when applied in the field has hindered the acceptance of bioaugmentation as a viable technology for in situ applications. These early attempts proved futile due to a lack of understanding of the specific requirements of the introduced cultures. For example, the competition between the indigenous and introduced microorganisms, limitations of microbial transport, transport of nutrient additions and electron-donor/acceptor substrates with the microbial culture, and the effects of the site geochemistry had not been investigated adequately at test sites. In addition, the results of some of the initial bioaugmentation field tests were ambiguous because those tests failed to employ experimental designs that provided sufficient control to allow a true test of the technology (e.g., it was difficult to separate the effect of introduced cultures from the effects of indigenous cultures, added nutrients, and dilution due to groundwater mixing). 
As the popularity of monitored natural attenuation/bioremediation remedies has increased in recent years, the incidence of failure of these technologies has also increased. In some cases, these technologies have failed due to inadequate site characterization and/or poor engineering design. In many other cases, however, these technologies have failed because the rate of biodegradation achieved by indigenous microflora is insufficient to meet remediation objectives. This is fueling the trend towards the consideration and application of bioaugmentation to improve the rate and extent of biodegradation processes when the capabilities of the indigenous microbial population are insufficient.

For site remediation, recent scientific and engineering involvement has led to advancements in the understanding of the underlying principals of bioaugmentation. Field applications are now under way with success being achieved. These successes are contributing to the advancement of the technology toward reliable and effective ex situ field applications. The latest development in the bioaugmentation arena is the successful demonstration of the technology for in situ treatment of contaminated groundwater. Particularly in the case of chlorinated solvents, several rigorous demonstrations have been completed recently that establish the principal design parameters for successful implementation of the technology in sand and gravel aquifers. Furthermore, a rigorous, longer-term and relatively large-scale demonstration of bioaugmentation, using the KB-1 culture, has been successfully demonstrated at the Caldwell Trucking Superfund Site in Northern New Jersey. This successful demonstration led to US EPA Region II allowing the initiation of a Focused Feasibility Study leading to a Record of Decision Amendment for the site.

The success of bioaugmentation has benefited, in part, from an improved understanding of the key microorganisms that mediate biodegradation reactions (e.g., Dehalococcoides ethenogenes in reductive dechlorination of cis- dichloroethene and vinyl chloride). For certain bioaugmentation cultures, the development and application of DNA-fingerprinting techniques now allows practitioners to detect and monitor augmented cultures in the subsurface with relatively high sensitivity. Through application of these molecular monitoring techniques, researchers are now better able to directly measure and distinguish the effect of bioaugmentation from the background processes. The recent success of bioaugmentation has also been made possible by advancements in the understanding of factors affecting transport, interaction, and survival of exogenous cultures in contaminated ground-water environments.

\subsubsection{Abiotic Processes}

The potential for enhancing abiotic degradation of CVOCs is currently unknown. The most promising approach appears to be a blend of microbiological and biological processes and is applicable to the anaerobic zone near the region of groundwater discharge where addition of sulfate (in the form of slowly dissolving gypsum) 
stimulates microbial reduction and generation of $\mathrm{H}_{2} \mathrm{~S}$. Reaction between $\mathrm{H}_{2} \mathrm{~S}$ and dissolved Fe(II) yields FeS, which then can react with and degrade CVOCs. This is an active research area with significant promise based on data from past MNA sites. In addition to abiotic degradation, there are opportunities to better quantify and to enhance other abiologic reactions (e.g. sorption).

\subsubsection{Phytoremediation}

The goals associated with enhancing phytoremediation are to 1) increase our fundamental understanding of the biochemical basis for phytoremediation, 2) explore strategies where increased transpiration can be used as an improved removal mechanism for CVOCs (by translocation to the atmosphere and direct metabolism) and to modify the large-scale hydrology of the system, 3) deploy available plants that maximize CVOC microbial destruction in the rhizosphere, and 4) enhance phytoremediation by genetic transformation to produce larger root mass and other favorable modifications.

To a large extent the utility of phytoremediation as a means of controlling the flux of contaminants in a dissolved phase plume is dependent on plant root interactions. Therefore, many enhancements to phytoremediation potential are fundamentally linked to efforts that improve favorable plant root properties. For example, plant roots are associated with the following processes:

- Uptake of water

- Uptake of contaminants

- Exudation of biochemicals (organic acids, etc.)

Processes that increase the surface area of contact of roots with soil and contaminants are likely to enhance water uptake and a spectrum of associated phytoremediation processes. For example, the main pathway for uptake of trichloroethylene (TCE) is passive via the plant transpiration stream. Beyond uptake and translocation of contaminants to the atmosphere, phytodegradation results from direct metabolism of CVOCs by plants. Therefore, the greater the rate of contaminant uptake the greater the amount of degradation that occurs through metabolic pathways. Another key role of plant roots may be in modifying the rhizosphere to promote microbial degradation of the contaminant, primarily through the production and release of organic acids and water-soluble carbohydrates (rhizodegradation).

There are several approaches that can be employed to increase the root mass and enhance its impact on phytoremediation.

Fertilization: Fertilization can be used to promote plant growth of existing species, improve microbial degradation, and modify soil chemistry (e.g. pH) in favorable ways. There are tradeoffs, however, as excessive fertilization can depress phenolic concentrations (e.g., salicylates) in plant tissues, as most of the organic solutes are 
required for biomass production and a lower proportion of the fixed carbon is partitioned into secondary carbon (phenolic) metabolism. However, increased dry matter production following fertilization increases carbon input from the turnover of fine roots, which increases carbon availability as well as the potential for increased organic acid exudation from roots. Nitrogen $(\mathrm{N})$ fertilization has a profound effect on increasing the flux in organic acid production, where the organic acids produced in the tricarboxylic acid cycle (Krebs cycle) are used as carbon skeletons to assimilate the applied $\mathrm{N}$ into amino acids. Fertilizer application needs to be timed to when it is most beneficial for plant-microbial interactions.

Selection of alternate species: Ideal plant species for phytoremediation 1) are widely adapted and fast-growing perennials (required for long-term phytoremediation of contaminated sites), 2) allocate a large fraction of assimilated carbon to root production and maintenance (required to occupy and affect large soil volumes), 3) produce and exude high concentrations of organic acids and water-soluble carbohydrates that can sustain the plant-microbe interactions, and 4) continue to function (as described in items 1-3) even under stresses, such as drought. A wide range of plant species has demonstrated varying degrees of phytoremediation capability, either directly on TCE or indirectly via stimulating rhizosphere interactions (Anderson et al. 1995).

Members of the genus Populus, in general, are fast-growing, woody perennials ideally suited for phytoremediation. Most taxa within the genus are cross-fertile, thus allowing numerous, broadly adapted hybrids to be created (Stettler et al. 1980, Dickmann and Stuart 1983). Hybrids currently exist that are tolerant of drought, high salt concentrations in soils, or periodic flooding (Tuskan 1998). A further advantage of Populus is that once selected, individual hybrids can be easily cloned, permitting a superior genotype to be propagated and utilized across a large number of sites. Furthermore, US DOE is sponsoring a large research effort that will elucidate the genome of Populus within the next year. This milestone will open new opportunities for selecting clones and generating transgenics that express key genes that will enhance phytoremediation capabilities.

Structural root systems of many poplar hybrids occupy and affect a large volume of soil, penetrating to depths of 6 to 12 feet depending upon the soil texture and depth to water table. Fine root turnover occurs at a rate of $\sim 0.5$ dry tons per acre per year (Dickmann et al. 1996). Fine root turnover is important because it ultimately increases soil carbon content (Hansen 1993) and may alter soil $\mathrm{pH}$, both of which are factors in sustaining soil microbial populations.

Genetic transformations: Genetic transformation offers the potential to improve a plant's ability to detoxify, sequester or isolate contaminants by modifying plant morphology or physiology. Increased root mass in genetically transformed plants enhances the production (quantity and quality) of secondary carbon metabolites and 
microbial activity in the rhizosphere, thus increasing the capacity of vegetation to translocate TCE and other VOCs from soils to aboveground plant components.

Genes responsible for detoxification of several organic contaminants have been identified. Phytoremediation using genetically-transformed plant materials currently includes the breakdown of trichloroethylene and other chlorinated halogens via naphthalene degradation (NAL) genes. Profound increases (640x) in the metabolism of TCE have been accomplished by the introduction of a gene with the enzyme oxidizing TCE, ethylene dibromide, carbon tetrachloride, chloroform and vinyl chloride (Doty et al. 2000). Other enzyme systems that result in TCE degradation can be identified in effective organisms, with the genes then similarly transferred into poplar clones to further enhance TCE degradation capability.

There is an issue related to the potential for unforeseen environmental consequences when the use of transgenic plants in the field is considered. These concerns have been addressed by the Animal and Plant Health Inspection Service (APHIS) of the U.S. Department of Agriculture. In general, if a field trial with transgenic plants is contemplated, notification must be submitted to APHIS for review and approval. If the duration of the trial does not extend to the point of flowering of the plant, then approval usually is granted. For longer field trials or commercial-scale releases, APHIS maintains a rigorous permitting process that must be completed before the transgenic plants can be released.

Some of the direct benefits of increasing root mass and root processes include:

Improvement of plant-rhizosphere interactions: Plants can be used as sources of soil carbon to enhance plant-rhizosphere interactions. For example, structural root systems of many poplar hybrids occupy and affect a large volume of soil, penetrating to depths of 6 to 12 feet depending upon the soil texture and depth to water table. Fine root turnover occurs at a rate of $\sim 0.5$ dry tons per acre per year, an important consideration because it ultimately increases soil carbon content and may alter soil $\mathrm{pH}$, both of which are factors in sustaining soil microbial populations.

There is a need to better understand what rhizosphere mechanisms allow plants and microbes to effectively process contaminants in soils. Carbon inputs to soils improve the environment for degradation of TCE by bacteria. The carbon input occurs both from the microbial-driven breakdown of plant tissues (decay of abscised roots and leaves), and from the direct exudation of organic solutes from living tissues. There is a need to determine the constitution of organic solutes in fine roots that are likely to be important in phytoremediation. Specifically, tricarboxylic organic acids, phenolic acids and water-soluble carbohydrates can support soil microbes.

Populus species and hybrid clones display great variability in the organic acids produced from root exudates. Examples include citric acid, malic acid, oxalic acid, 
salicylic acid, and their higher-order phenolic glucosides that are complexed with caffeic acid and benzoic acid (populin, tremuloiden, tremulacin, salireposide, salidroside, etc.) (Tschaplinski and Tuskan 1994, Tschaplinski and Blake 1994, Tschaplinski and Blake 1989a,b). The function of hybrid poplar in phytoremediation has been largely attributed to the production of these organic acids and phenolics released as root exudates when roots are exposed to contaminated environments, but details of which clones and which specific compounds are most stimulatory of plantmicrobial interactions that foster TCE breakdown are not well known. Prudent clone selection can be used to maximize production of those organic acids, water-soluble carbohydrates and phenolic compounds that will enhance microbe availability and TCE metabolism.

Transpiration as a hydrologic control mechanism: Another potential benefit of large root mass and high transpiration rates is the ability of trees to alter water infiltration fluxes and to manipulate the shape of the water table that can result in changes in the location and geometry of contaminant plumes. Consumption of 30-54 inches of water per acre per year by some hybrid poplars may be sufficient to have a significant effect on subsurface soil moisture and to modify groundwater flow and contaminant migration in predictable ways.

One approach is to use plants to control the flux of infiltrating recharge through the vadose zone. This has been suggested as a method for reducing the dissolution rate and associated flux of contaminants from a CVOC source in the unsaturated zone. A second approach is to use the high transpiration rates of trees such as poplars to modify the shape of the water table. It may be feasible to use this strategy to create hydrologic barriers to influence contaminant migration pathways in desirable ways. For example, it may be possible to use this strategy to reduce the zone of discharge to surface streams or wetlands and to optimize placement of other treatment and monitoring facilities.

\subsubsection{Active Processes at System Interfaces - Potential Role in MNA/EPR}

Figure 7 is a simplified diagram of a generic site where a source of chlorinated solvents in the vadose zone migrates downward to the groundwater. The vadose zone ("I") and the other major zones along the plume trajectory are designated sequentially using roman numerals. Once in the groundwater ("II"), the plume moves along a curvilinear path, controlled by the large scale sources and sinks of subsurface water and the geological structure toward the downgradient wetlands ("III") and stream ("IV"). If the natural attenuation capacity in zones I through III does not match or exceed the contaminant loading from the source, the plume will crop out into the wetland and/or stream system. The location of the source, the relative thickness and geometry of the various zones, and specific geologic features control the specific location and the nature of the transition from the subsurface into the surface water system (hyporheic, riparian or fluvial). Several versions of this simple paradigm in 
which the nature of the source and setting are varied have been examined in developing and prioritizing critical science associated with natural attenuation - all of the versions, however are represented in a general sense by the generic diagram below.
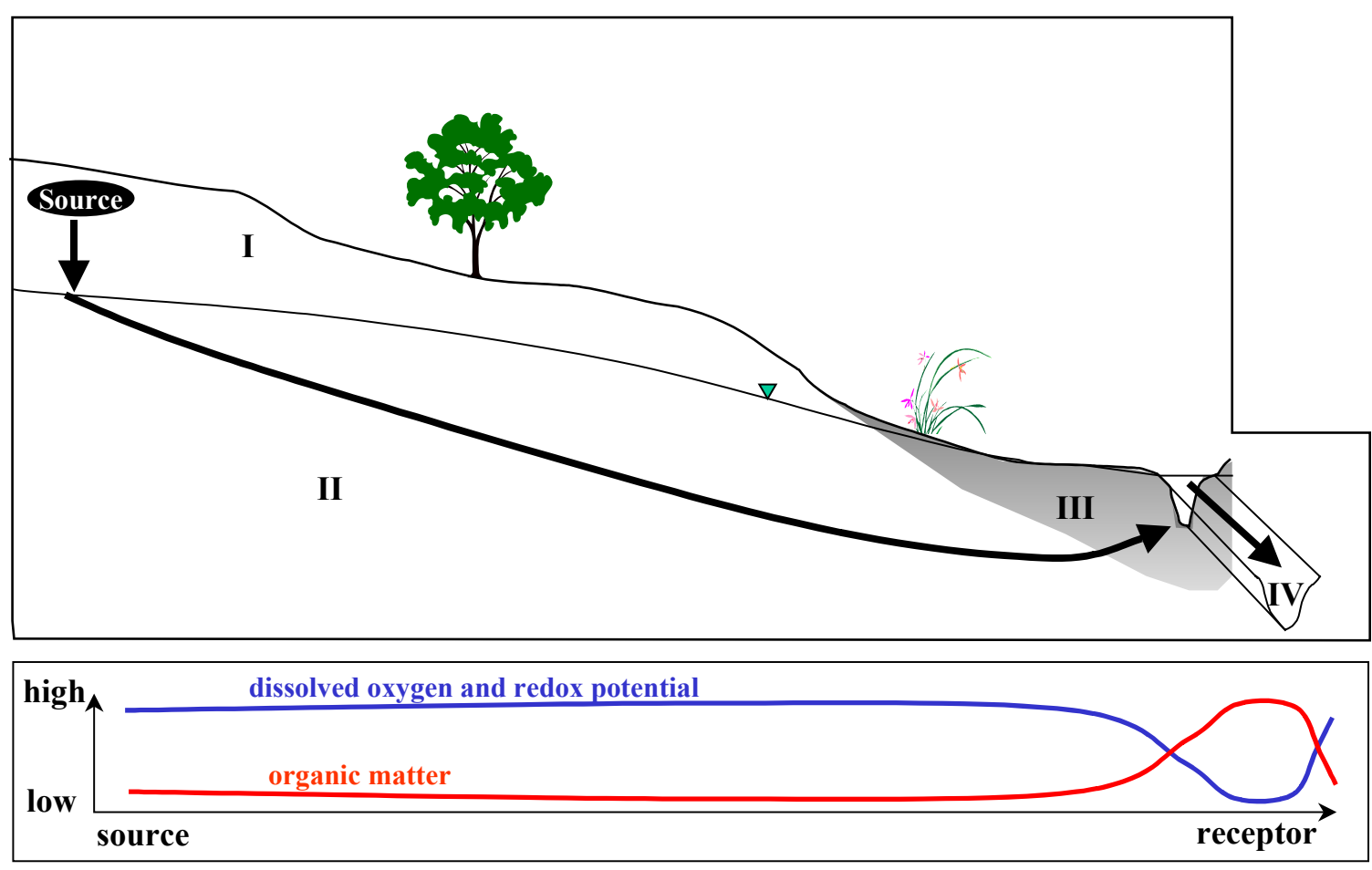

Figure 7 Simplified schematic diagram of the major zones potentially traversed by a chlorinated solvent plume. $\mathrm{I}=$ vadose zone, $\mathrm{II}=$ groundwater, $\mathrm{III}=$ "wetland", IV = stream.

The landscape model described above lends itself to a qualitative examination of processes and identification of relevant scientific literature. In particular, there has been substantial interest in the relatively active processes occurring at the interfaces of the major compartments: the vadose zone groundwater interface (I-II) and the groundwater surface water interface (II - III/IV). Of these, the groundwater to surface water zone appears to be the most promising for study of natural attenuation and remediation. This promise results from the high biomass associated with active biological processes that often occur in wetlands and groundwater discharge zones and from the rapid shifts in geochemical conditions. This is generically represented in Figure 7 using a graph of landscape-level geochemical gradients across the system. The conditions at system interfaces in many systems may be conducive to 
degradation processes and the degradation in the vicinity of the interface may dominate the natural attenuation capacity in the entire system. Study of interfaces is particularly useful for examining the relative importance of landscape-level processes and the hydrologic processes that deliver contaminants to the interface. For example, an upland source located closer to the wetland interface would arrive on a flowline that is higher in the aquifer than the one depicted and would encounter the groundwater wetland interface earlier and would interact with the system differently. What are the implications of such questions of simple plume structure and geometry? Similarly, Figure 7 suggests sustainable enhancement possibilities for implementing EPR. If the wetland system (III) shows the presence of active degradation processes but the capacity is insufficient for the loading, then the system could be enhanced by physically expanding the wetland to the left and/or by increasing attenuation capacity through a shift in biomass or community structure.

In this line of inquiry, studies of processes at these large scale interfaces were examined. Several specific scientific papers were summarized on topics ranging from phyto-uptake and transpiration of contaminants to the geochemical gradients in typical systems. Importantly, key findings from past national reviews of processes that occur in interfacial zones were cited and presented (see, US EPA 2000 and Sophocleous 2002).

Several opportunities were identified as possible technical targets to support the US DOE MNA/EPR development and acceleration effort:

Expand Groundwater Surface Water Interface for sites where associated attenuation capacity is present but needs to be increased to match loading Create New Interfaces within the landscape for increasing overall attenuation capacity (e.g., sustainable permeable reactive barriers (PRBs), implementing a sustainable aerobic fringe to stabilize plume)

Exploit the Vadose-Groundwater Zone Interface by employing a long lived light nonaqueous phase liquid (LNAPL) to intercept and destroy contaminants as they exit the vadose zone

Exploit Volatilization from Surface Water -- for plumes that discharge by accounting for and maximizing water to air transfer (role of volatilization/photodegradation, definition of plume stability, allowable loading, and overall system boundaries)

\subsubsection{Summary for Large Scale Hydraulic Modifications}

Most of the concepts for EPR rely on adding reactants, organisms or infrastructure to a system to increase the attenuation capacity of the system so that it is sustainable and exceeds the contaminant loading. Alternative concepts based on large-scale hydrologic modification include: 1) reducing the contaminant loading so that the natural attenuation capacity of the site is sufficient, and/or 2) to reduce the entry of competing electron acceptors into the contaminated area to reduce competition for 
existing electron donors. There are a variety of different approaches that are possible to achieve this goal. Most of these approaches rely on reconfiguring the site to permanently modify the large-scale water balance. Because "loading" is the product of concentration and flow, this term can be directly modified by altering and reducing the flow of water. Further, setting up permanent, sustainable, and easily documented changes in large-scale hydrology is relatively straightforward. Flow controls will be in place as long as the hydraulic controls are in place.

Hydraulic controls attempt to prevent contaminated water from reaching certain regions of the aquifer or to prevent clean water (or water containing competing electron acceptors) from contacting contaminated zones. Hydraulic controls alone are often not considered in evaluating options because they do not destroy or immobilize contaminants. When considered in the context of MNA and EPR where the goal is to maximize capacity and minimize loading, this concept appears promising. Implementation can take many forms including the traditional variants (Rumer and Mitchel, 1995): "capping near the source", "cutoff walls", and "building a dam." There are other viable concepts that may perform as well as these highly engineered systems at a lower cost. Alternative concepts to dams, walls and caps include:

"passive collection and bypass of clean upgradient water using a siphon or drain", and "increased runoff and evapotranspiration throughout the plume area". While not yet widely implemented, these approaches are receiving increasing attention as US DOE is developing strategies for sites that are contaminated with chlorinated and radioactive contaminants. Recent examples include technical recommendations for plumes at the US DOE Kansas City Plant and the Ashtabula Closure Project.

At some sites, a second benefit can be realized by one form of large-scale hydrologic modification. This second benefit, electron acceptor diversion, may be at least as important as the reduced loading effect at some chlorinated solvent sites. To accelerate the natural dechlorination process for the purpose of bioremediation, numerous research groups have focused on methods to increase the supply of electron donors to dechlorinating bacteria. Most researchers and technology developers add electron donors (such as lactate, molasses, mulch, etc.) that ferment in-situ to release hydrogen. An evaluation of the electron donor mass balance at sites where reductive dechlorination occurs suggests another approach for increasing the net electron donor supply to the dechlorinators. By employing a large-scale hydrologic barrier, it is possible to permanently divert the transport of competing electron acceptors (oxygen, nitrate, and sulfate) away from chlorinated solvent plumes. This allows more electron donor (i.e., organic substrates and/or dissolved hydrogen) to be preserved for beneficial reductive dechlorination reactions (Newell et. al, 2001a, 2001b; Newell, Aziz, and Cox, 2003).

In summary, modification of hydrology may provide significant benefits and may be straightforward to implement and monitor. The two benefits investigated in this line of inquiry were: 1) the loading to the plume will be reduced; and 2), competing 
electron acceptors will be diverted away from the source zone, thereby increasing the rate of naturally-occurring bioremediation and increasing mass destruction of chlorinated solvents. Such a hydrologic diversion system would be relatively inexpensive, reliable, and have the potential to significantly enhance natural attenuation processes in a passive manner. Construction of a system might result in the collapse of the dissolved plume downgradient of a source zone, even if the plume were expanding prior to construction.

\subsubsection{Scenario Tools for Evaluating MNA}

There are a number of strategies available to simplify the implementation of MNA/EPR. One promising research area is the classification of chlorinated solvent sites into different scenarios based on factors such as parent compound, plume stability, presence of biotic reactions, presence of abiotic reactions, hydrogeologic settings, source type, and other factors. This approach is similar to a taxonomic key approach in biology, where classification based on a handful of key variables provides a powerful framework for understanding key concepts. MNA/EPR scenarios would help the user understand how the site works and what needs to be done to evaluate MNA/EPR.

The application of scenarios to MNA/EPR for chlorinated solvents is somewhat challenging. The primary reasons for this is the explicit recognition that each site will be unique, that MNA/EPR might be appropriate and applicable under a wide range of scenarios, and that MNA/EPR is unlikely to always be applicable under any one scenario. Nonetheless, when viewed in terms of a taxonomic key leading to a portfolio of twenty or so scenarios, the process appears tractable and useful. Such a key would help guide what to measure and where to measure for a particular and relatively narrow class of sites. Early use in a characterization and monitoring process appears particularly useful to help efficiently focus resources. It is both important and valuable to add such structure to the multiple lines of evidence approach. As a result, some form of scenario concept should be encouraged.

For MNA/EPR, a scenario-based approach could be based on detailed descriptions of 10-30 generic types of chlorinated solvent sites ("scenarios"). Users would navigate through simple flowcharts of key distinguishing factors, and then select the scenario from the portfolio that best matches their own site. The flowcharts would serve as the taxonomic key to help simply identify the appropriate scenario - much in the same fashion as a field guide to identify plants or animals. Using a 2-4 page fact-sheet format, the selected scenario would then provide the user with the following information:

- Conceptual model graphics of chlorinated solvent plumes;

- Key MNA/EPR processes that are likely active at the site;

- Degradation pathway diagrams; 
- Observed range of degradation rate constants;

- Recommended approach to show key geochemical footprints;

- Lines of evidence that should be employed;

- Key monitoring parameters for MNA/EPR Process Monitoring;

- Key monitoring parameters for MNA/EPR System Performance Monitoring;

- Recommendations for configuration of the monitoring system;

- List of key figures and graphs needed to demonstrate MNA/EPR;

- Appropriate computer models for this type of site;

- Applicability of emerging monitoring techniques;

- Applicability of various enhancements. 


\subsection{Documenting and Monitoring MNA and EPR}

\subsection{Overall Characterization and Monitoring Strategy for MNA and EPR}

Remediation alternatives that rely on natural mechanisms ultimately depend on the attenuation capacity of the system - the sum of the various mechanisms must be sufficient to attenuate the contaminants and protect potential receptors. The goal of characterization is to provide evidence that the attenuation capacity in the system is sufficient to off-set or balance the contaminant loading on the system in a reasonable period of time, Figure 8 . The goal of monitoring then is to verify that the attenuation capacity is maintained over time and that if conditions vary the attenuation capacity will be maintained until remediation objectives are met.

The strategy for MNA characterization should be based on cost-effectively determining the contaminant loading of the system by quantifying and tracking the contaminants and balancing this against the attenuation capacity of the system determined by estimating the contribution of each attenuating mechanism in reducing the contaminant concentrations. Monitoring verifies that the attenuation capacity is maintained over time.

Importantly, faithful adherence to this concept may encourage innovative monitoring techniques and configurations to reduce costs while maintaining or improving performance and protection. Baseline approaches, along with a variety of alternative techniques and configurations, will be examined in the characterization and monitoring Lines of Inquiry. The potential value of the various concepts will be critically explored.

\subsubsection{Background}

Every natural system has some capacity to attenuate environmental contaminants through a variety of physical, chemical and biological processes. Characterization should be designed to identify and estimate the contribution of each "significant" attenuating mechanism, thus providing the basis for determining if monitored natural attenuation or enhanced passive remediation is appropriate for a site. If natural attenuation mechanisms are shown to be acting at a rate sufficient to meet regulatory targets, monitoring is needed to verify and validate that the identified natural attenuation mechanisms are proceeding as predicted. If natural attenuating mechanisms are not sufficient, an assessment of the system should be made to determine if the natural mechanisms may be enhanced to provide sufficient capacity in the system. 


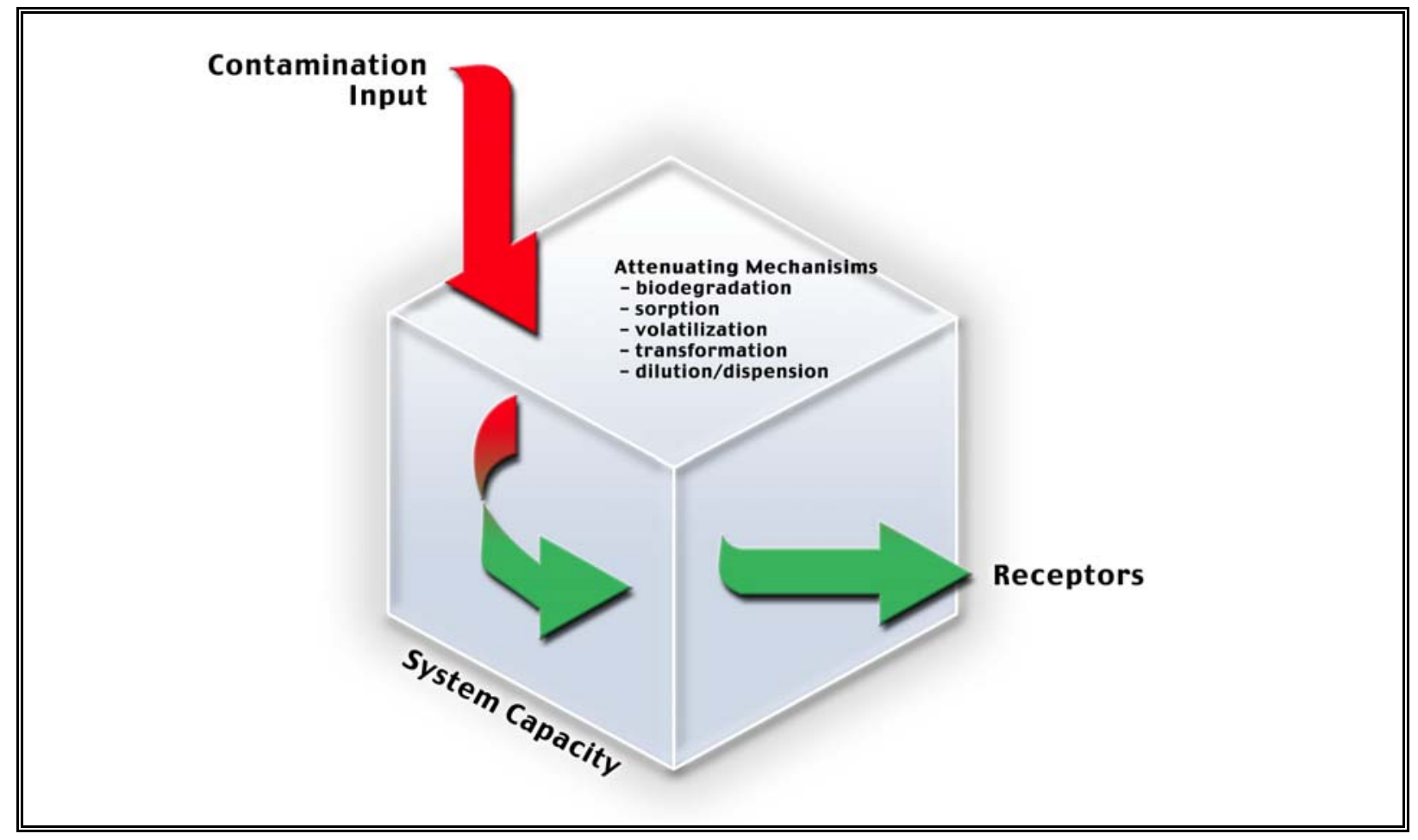

Figure 8 Mass balance between contaminant loading and system capacity

The first formal recognition of the natural attenuation of chlorinated solvents can be traced to the "Natural Attenuation of Chlorinated Solvents" course in Austin, TX, September, 1997. The Industrial Members of the Remediation Technologies Development Forum (RTDF) Bioremediation Consortium developed the curriculum and prepared this training course and associated workbook in cooperation with the Inter-State Technology Regulatory Council (ITRC). Following these training courses, the US EPA protocol (1998) and subsequent guidance (US EPA, 1999) acknowledge, in general, the level of site characterization and monitoring necessary to support a comprehensive evaluation and application of MNA requires a higher level of detail and effort than is generally required for engineered remediation options. For characterization there is a significant burden of proof because many of the natural attenuation processes such as biological activity and flux can not be measured directly, but only inferred from other parameters. As a result, the concept of multiple lines of evidence was developed to validate the use of MNA. The ability to directly measure these parameters could be a significant advancement for MNA. The US EPA directive (US EPA, 1999) also acknowledged that although performance monitoring is a critical element of all response actions, performance monitoring is of 
even greater importance for monitored natural attenuation. This is due to the potentially longer remediation timeframes, potential for ongoing contaminant migration, and other uncertainties associated with using monitored natural attenuation (US EPA, 1999). If traditional monitoring techniques are used for the duration of the remedy, the costs can potentially "price" natural attenuation out of contention with other remediation options. Paradoxically, monitored natural attenuation is often considered to represent a cost-savings over more active treatment techniques. The inability to implement effective, but reasonably priced characterization and monitoring is one of the biggest barriers to the implementation of monitored natural attenuation.

Subsequent to the development of the US EPA protocols (US EPA, 1998), relevant scientific and technical advances have been made on diverse topics ranging from geochemical conditions as they relate to attenuation potential, to bioassessment tools. These various developments along with recent innovations in characterization and monitoring will be critically evaluated for incorporation within the characterization and monitoring lines of inquiry.

\subsubsection{Approach}

The approach to characterization and monitoring of MNA/EPR is predicated first on the remediation goals for the site, and secondly on the system's mass balance or the system's ability to attenuate the contaminant. The remediation goals are critical to define up front and will be used to define the boundaries of the system. For example, if the goal is for contaminant concentrations to be lower than a maximum concentration at a compliance point downgradient, the system will be defined and bounded between the contaminant source and the compliance point. Once the system has been defined, the attenuating mechanisms operating within those boundaries can then be determined and an estimate of the total capacity, rate and sustainability of those processes can then be estimated.

Ideally, in characterization, the attenuating mechanisms should be directly determined, but currently, in most cases this is technically infeasible. Only indicators of the actual processes can be measured and the attenuating process inferred. This complicates the process in two ways. First, the case for natural attenuation must be built on multiple lines of evidence to build a compelling argument that the attenuating mechanisms are operating. Second, determining what parameters are needed and how they will be used complicates the characterization process. For example, in order to assess the anaerobic degradation of chlorinated solvents it would be ideal to directly measure the metabolic rate of the microbes degrading the contaminant, however, currently this is not technically feasible. Only indicators of this process may be measured; e.g. dissolved oxygen concentrations, byproducts of the metabolic process such as carbon dioxide and the presence of a food source in carbon, etc. If enough of these parameters are favorable, then the attenuating mechanism can be validated. 
The structure and concept for the scientific and technical efforts to advance characterization and monitoring will be organized into four transitional phases:

screening characterization, decision characterization, process monitoring and system verification. We believe these four phases will be applicable regardless of the regulatory process, US EPA or state, under which the site is being cleaned up. In the paragraph below we discuss the four phases as they would apply to the CERCLA process. These four phases are presented in Figure 9 with descriptions of key components of each phase. The lines of inquiry will be developed under each phase based on 1) review of the state of practice and the state of science, and 2) critical evaluation of the potential benefits of different types of activity.

These four transitonal phases overlay the CERCLA process with the intent to expand the description of characterizations and monitoring and not to replace nor increase the requirements of the Remediation Investigation/Feasibility Study (RI/FS). In particular, the first two phases, screening characterization and decision characterization are part of the CERCLA RI/FS. The initial assessment of MNA, the

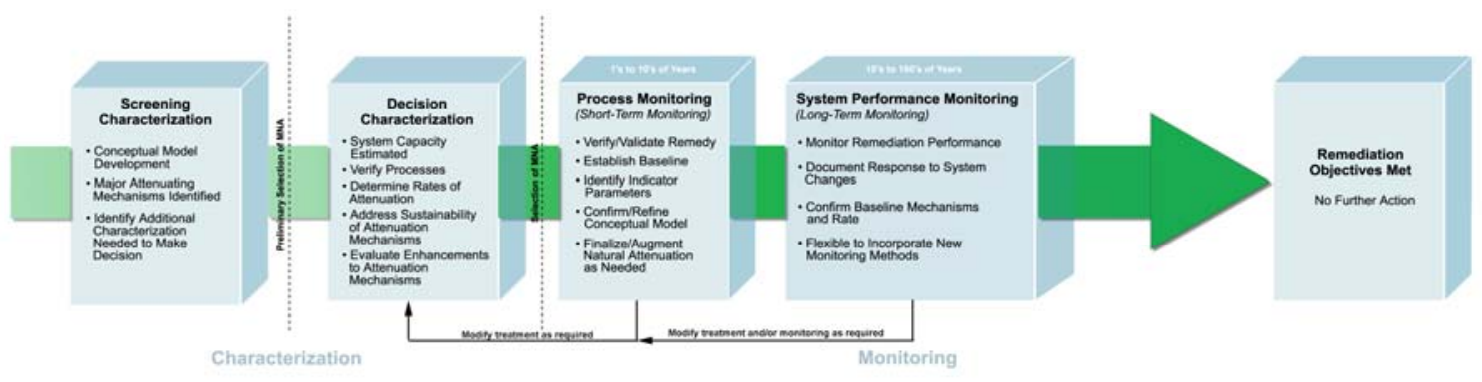

Figure 9 Characterization/Monitoring Transition

screening phase, begins during the later stages of the RI and continues through the development and screening of all technologies identified in the FS. The decision characterization coincides with "treatability investigations" under the CERCLA process. These investigations are undertaken when additional data is needed to evaluate technology performance for the Feasibility Study.

The process monitoring and system verification phases are designed to carry MNA through to the final disposition of the site, and begin once the Record of Decision (ROD) has been signed. Under the CERCLA process, implementation of the remedial action and any monitoring associated with that are identified in the ROD. Changes to the ROD can be incorporated during a standard 5-year review cycle. For MNA, perhaps more than for engineered solutions, verification that the attenuation capacity is maintained over time and as conditions vary until remediation objectives 
are met is a critical aspect to implementation. The process monitoring and system verification phases provide the framework from which the remedy may be applied through the CERCLA process.

\subsubsection{Lines of Inquiry}

The lines of inquiry were determined by the Technical Working Group and are aligned under each of the major characterization and monitoring phases with the core values embodied in the following list:

- Document performance using the mass balance of system capacity and contaminant loading concept

- Refine and streamline characterization and monitoring

The overarching goal of the characterization and monitoring developments will be to facilitate MNA/EPR by reducing costs while maintaining or enhancing the information available to document that the system is protecting the public and the environment.

The following is an outline of the lines of inquiry and specific examples of science and technology to be evaluated. Importantly, the list includes a relatively comprehensive list of ideas that are being examined in current research programs, but all of these were not determined to be promising or appropriate to incorporate into the "next generation" protocol. Determination of potential value of any concept was a primary function of the critical examination by the Technical Working Group and was the basis for the prioritization of the relative value of the various concepts to facilitating safe and appropriate use of MNA/EPR.

Multiple Lines of Evidence: Incorporate latest research and scientific logic to enhance the existing multiple lines of evidence concept. Currently, direct measurement of attenuating processes is technically challenging which necessitates that a preponderance, or "quorum," of evidence be collected to support the use of MNA. The people that review and approve MNA must establish the definition of an acceptable quorum. Develop a paradigm that includes some of the spatial process mapping and other items highlighted in the NAS review of the previous protocol. Included also, is the concept of a responsive characterization process based on conditional rules (i.e., no need to measure reduced gases at sites with measurable dissolved oxygen).

Bioassessment tools: In an effort to directly measure some of the more important attenuating mechanisms for chlorinated solvents (e.g. microbiological degradation), a line of inquiry into bioassessment tools was developed. These include nucleic acid probes, fatty acid profiles, taxonomy, 
structure and function screening profile systems, fluorescence methods, and other tools. Other bioassessment tools include hyperaccumulators (possibly coupled with remote sensing) and macrobioaccumulators (clams, etc.), and biomarkers (ecosystem structure and species composition). These latter tools integrate exposure and may provide a more realistic measure of impact.

Sensors: Determine the value of sensors in characterization and in monitoring MNA/EPR systems. Examine the need for sensors that provide high frequency data. Examine alternative configurations that use on-off sensor signals rather than concentration signals as a way to reduce costs. Evaluate passive and cumulative sensors that would act similarly to bioconcentration. Emphasize "system" monitoring and large-scale design and monitoring concepts particularly in the later "long-term" system performance monitoring phase. Examine integrating measures, such as contaminant flux, geochemical footprints (NRC, 2001), and other averaging and volumetric methods in monitoring. Incorporate latest research on remote sensing, geophysics, and flux monitoring. This includes both instrumentation and interpretation and deployment options (horizontal wells, lidar, remote sensing, and others). Examine lessons from agriculture and soil science ("smart farming") and potential for cross over applicability. Incorporate the latest research on state-of-the-art sensors.

Non-traditional Monitoring Systems: Incorporate the latest research on surrogate measures to reduce costs. These include bulk and master variable properties such as redox potential, as well as indicator species (e.g., $\left.\mathrm{Cl}^{-}\right)$and specialized tools such as total halocarbons, degree of chlorination sensors, etc. Incorporate the latest research on monitoring system configuration. This includes focusing monitoring on designed or identified monitoring points (e.g., weak points that would serve as indicators of performance throughout the system) and focusing monitoring at interfaces.

Modeling: Incorporate latest research on bioinformatics and modeling. This includes data mining, neural networks, incorporating new types of data, integrating diverse types of data, working at sites with large amounts of data and determining the value of data to justify reducing the number and frequency of analyses. Consider the latest progress in both forward and inverse predictive modeling and the potential value of large-scale mass balance models (i.e. simple balancing delivery and treatment capacity) as an alternative that might be used at many sites.

Figure 10 shows the relative importance of each line of inquiry to the characterization and monitoring phases. Underlying all of these phases is the conceptual model that will be continually refined and updated as new data come available throughout each of these phases. 

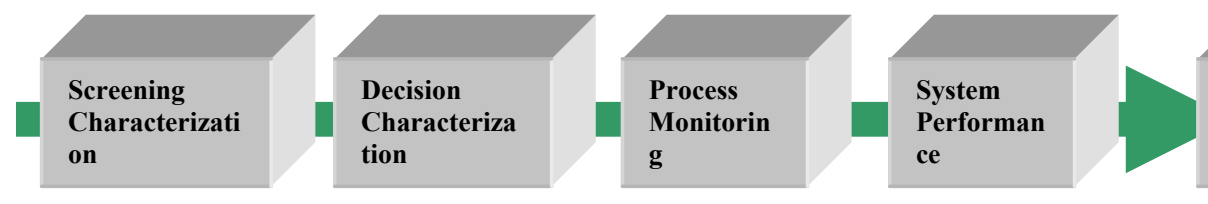

Remediati on Objectives

\section{Monitoring Configuration}

\section{Sensors}

\section{Lines of Evidence}

\section{Bioassessment Tools}

Unconventional Monitoring

\section{Modeling}

\section{Figure 10 Relative importance of each Line of Inquiry at each phase of the characterization and monitoring process.}

At the outset of assessing the use of MNA/EPR, as with all remediation assessments, a set of remediation goals should be developed. These goals will be used to define the "system" in order to provide the parameters for determining the system's capacity as well as provide milestones or decision points to manage the characterization and monitoring process.

\subsubsection{Characterization}

The approach for the characterization and monitoring of a site for MNA/EPR first requires definition of goals and then requires a preliminary estimate of the natural attenuation capacity. In this screening characterization phase a preliminary understanding of the primary attenuating mechanisms and where in the system these processes are active will be developed. It will also be important in this initial phase to use the data to develop a site conceptual model taking into account both spatial and temporal variations. This conceptual model will guide future characterization and monitoring efforts and should be continuously updated and refined throughout the characterization and monitoring process as additional data becomes available. At some sites this screening characterization phase may be straight forward, particularly where one or two primary attenuating mechanisms provide sufficient system capacity to degrade contaminants. Other sites may be more complicated or it could be determined that MNA is not technically feasible. The goal of this phase is to 
determine if MNA is potentially viable at the site and warrants more detailed characterization to make the final decision. This is in line with the US EPA protocol (US EPA, 1998).

The screening phase, is followed by the decision characterization phase in which the capacity and loading or mass balance of the system is determined by identifying what mechanisms are active, their rates, and an estimate of the sustainability of the processes involved. This more detailed characterization is addressed in the Lines of Evidence section below where a sufficient case must be built to support MNA. Recent advances in the scientific basis of the various attenuating mechanisms has been captured in this section to include some of the abiotic degradation processes which at some sites may be an important attenuating mechanism. The decision characterization phase leads to a decision point related to whether natural attenuation is a viable remedy for the site. The decision could be to go forward with MNA, or that the attenuating mechanisms need augmentation to increase the capacity and reach a mass balance. Depending on the data, additional characterization needs might be defined, or it may be determined that MNA is not viable and enhancements are not possible - in this case an active remediation technique will likely be needed to meet the remediation goals.

\subsubsection{Monitoring}

If monitored natural attenuation is selected as a remedy, the characterization phase transitions into a verification and validation phase referred to as "process

monitoring". In this phase attenuation capacities and rates are confirmed, data are collected to establish a data set that verifies the process is continuing and indicator parameters are identified to streamline data collection in the long-term. The final stage of monitoring, the "system verification" phase will in many applications be the longest in duration. It will therefore be important to make the monitoring process as efficient as possible while still confirming baseline mechanisms and rates, and documenting responses to changes in the system. To accomplish this, the focus of this phase should be on the system as a whole. For example measuring flux rates or documenting stresses to the ecological system as opposed to point measurements in wells. Indicator parameters identified in the process monitoring stage may also prove useful. This phase of monitoring necessitates the development of alternative monitoring techniques and configurations to cost-effectively meet the objectives.

\subsection{Multiple Lines of Evidence}

The purpose of this line of inquiry was to explore possible enhancements and improvements to the existing guidance for collecting multiple lines of evidence to support decision-making for MNA as a remedial option. Using the concept of natural attenuation capacity with its multiple processes, one must be able to evaluate which of these processes are occurring and to what extent. Because there are multiple 
processes of natural attenuation, it is important to identify the predominant processes occurring within the system. Then a "quorum of evidence" is assembled to understand the extent and rate at which these processes are occurring and if they are sustainable to handle the loading of contaminants into a given treatment "zone". If the processes are not sufficient or sustainable, then data needs to be collected to evaluate if there are ways to manipulate the system to make them sustainable. The difficulty lies in the ability to measure these processes. To measure these processes, indirect methods are typically employed. An analogy used in the Natural Processes section of this document describes measuring the processes as painting a picture of a person using the shadow as the model. Thus, to accurately paint the person or define the process, data must be collected from different "angles." The data collected through the multiple lines of evidence approach allows the technical person to evaluate the attenuation processes occurring and identify the predominant ones, if necessary. The concept of multiple lines of evidence compliments the process of determining sustainability of MNA followed up by identification of EPR techniques when the system is not self-sustaining.

The use of multiple lines of evidence has been documented by US EPA in both OSWER Directive 9200.4-17 (1999) and US EPA Technical Protocol for Evaluating Natural Attenuation of Chlorinated Solvents in Groundwater (1998). Multiple distinct but converging lines of evidence, in various forms have been used in recent years to evaluate natural attenuation (National Research Council, 1993; Wiedemeier et al. 1995, 1996a, and 1999, US EPA, 1998 and 1999b, and ASTM, 1998). US EPA identified three lines of evidence to estimate natural attenuation. These are: 1) historical groundwater/soil chemistry data to evaluate contaminant mass loss over time (flux), 2) hydrogeologic and geochemical data to evaluate the type and rate of biological processes occurring at the site that may reduce the contaminant concentrations, and 3) field or microcosm studies which directly demonstrate the occurrence of a specific natural attenuation process. The guidance in OSWER Directive is to build a case of evidence starting with the first line of evidence and progressing through the third line as needed to provide adequate and conclusive evidence that natural attenuation mechanisms are occurring. The guidance in the US EPA technical protocol provides detailed guidance for implementing the OSWER Directive, specifically for evaluation of biological degradation of chlorinated solvents, including a comprehensive list of analytes for analysis.

The most promising enhancements to the current lines of evidence concepts as identified by US EPA are inclusion of analyses to indicate abiotic degradation as a degradation process associated with natural attenuation, inclusion of the molecular tools for bioassessment into the degradation processes analyses, and inclusion of additional logic to help organize and select among the analyses identified by US EPA in the Technical Protocol document (1998). 
Recent advances in the understanding of chlorinated solvents shows that abiotic reactions can be important in some systems. Thus, inclusion of the analyses that lead to a determination of whether abiotic processes have a prominent role in natural attenuation is warranted. New molecular tools, as discussed in the molecular tool section of this document, have added greater capabilities for the analyses of microbes associated with natural attenuation degrading processes. These are discussed and included as tools for use in developing the lines of evidence.

The third enhancement of adding logic to analyses for determining degradation processes associated with natural attenuation will help the technical person proceed with the characterization of natural attenuation processes in a systematic manner. Because direct measurements are often not available to define the operative attenuation processes, it is not intuitive how the long list of analyses identified in available protocols is to be used and interpreted. "Why are the data being collected?" "How should the data be interpreted?" "What of the optional parameters should be collected?" The purpose of creating the logic is to help the technical person to evaluate the data that is collected and determine how to proceed.

\subsection{Characterization and Monitoring Opportunities}

\subsubsection{Advanced Bioassessment Tools}

Current MNA practices have relegated traditional microbiology approaches (e.g. classical direct detection of specific microorganisms, characterization of microbial communities, and processes and rates via microcosm studies) to the optional or "third line of evidence", a supporting role and used only if absolutely required to confirm processes derived from geochemical footprints. This supporting role is due to general assumptions that chlorinated solvents will degrade if the right environmental conditions exist, and that microbial identification and quantification is an expensive and time-consuming activity. Furthermore, there is concern on the validity of extrapolating laboratory degradation rates to the field.

Advances in technology and scientific knowledge over the last five years have produced a new set of bioassessment tools that now can provide a direct line of evidence for MNA. These advances include: increased understanding of various degradation processes and their contribution to overall attenuation capacity; isolation of key microorganisms and recognition that they are not ubiquitous in the environment; and advances in molecular (genetic) technologies that allow rapid and direct measurement of specific microorganisms, the structure of microbial communities, and metabolic functions in soil and groundwater samples.

Direct measurement of microbial species, communities and their metabolic functions is now possible and has several distinct advantages over indirect measures of 
microbial activity (i.e., production/disappearance of various electron donors and acceptors, or bioindicator parameters). Advantages include:

- Assessment of current and future potential to degrade chlorinated solvents: Bioindicator parameters infer the type of environment in which degradation processes may occur, but directly indicates if the requisite microorganisms for chlorinated solvent biodegradation are present and active (e.g., strains of Dehalococcoides that are required to convert cDCE past VC to ethene, known aerobic cDCE degrading bacteria). Documenting the distribution of key microorganisms downgradient of an expanding plume can be used to show "future potential or capacity" of a system.

- Spatial distribution of capacity or potential: Bioindicator parameters are transportable (e.g., migration of methane, ethene, degradation products, or "shadows" of compounds depleted with the plume), and therefore, limited in assessing the spatial distribution of various degradation activities. Assessing the capacity of a "MNA block" requires knowing its true spatial dimension, which can only be ascertained confidently by knowing the distribution of key microorganisms.

- Direct measurement of degradation rates: There are two molecular methods that can be employed; detection and quantification of specific microorganisms (using PCR), or detection and quantification of functional genes using DNA microarrays. The approach of detecting and quantifying specific or groups of microorganisms relies on the general correlation between the amount of microbial biomass and the total degradation rate of a compound (e.g., $\mu$ moles/g cell protein/day). As the total biomass is also a function of available nutrients, measuring biomass also reveals the flux of rate limiting acceptors and donors to the microorganisms, thereby reducing the need to collect this type of data or improve the interpretation of it. A current limitation of PCR-based approaches is that not all species have been identified that are responsible for various degradation processes for chlorinated solvents (e.g., anaerobic oxidizing bacteria). This limitation can be overcome by the use of DNA microarrays that can be used to identify specific functional genes that are invoked when the chlorinated solvents are being degraded. These microarrays can hold thousands of genes and can be used to assess which degradative genes have been expressed, and the amount of expression (related to the level of activity or degradation rates). Each of these methods would allow mapping the spatial distribution and evaluation of temporal changes of field degradation rates better than bioindicator parameters. This is simply because the measurements are relatively independent of transport processes; bioindicator parameters require assumptions or measurement of dispersion/dilution processes to derive degradation rates.

- Improved performance monitoring: Any spatial or temporal changes in biomass of key microorganisms or changes in functional gene expression can be used to monitor MNA performance (i.e., change in capacity), or as indicator flags. Furthermore, the samples can be archived indefinitely, and therefore can be reanalyzed at any time in the future and compared to current conditions. In some 
cases, bioassessment tools can be used to monitor the disposition and function of microbial activities resulting from biostimulation and/or bioaugmentation.

Molecular detection technologies now provide us with cost-effective tools to directly detect, assess, and monitor biological processes with greater confidence and less uncertainty than current indirect measurements. These tools also have the potential to reduce the number of parameters required for MNA evaluations, and can be easily integrated into MNA evaluations as presented in Figure 11. Furthermore, we will continue to derive benefit from the continued rapid advancement of these technologies in the medical and research fields that is further driving down unit costs, improving sample throughput, and increasing automation.

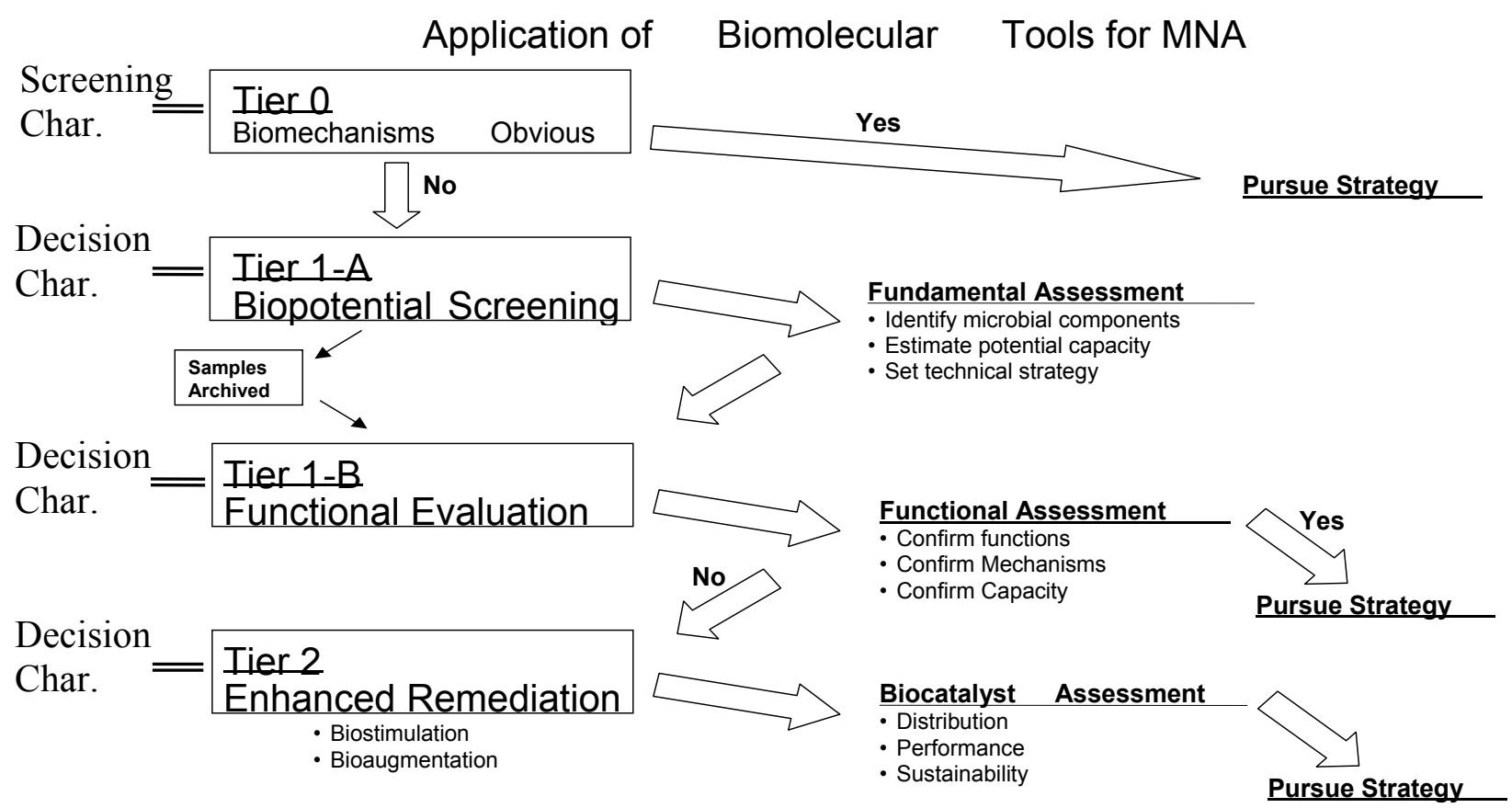

Figure 11 Application of Biomolecular Tools for MNA

As with the techniques and analyses that provide indirect measures microbial activity, guidance must be provided for these new technologies that provide direct measures of microbial activity. This guidance will provide the practitioner and regulator with information on what these techniques measure, information on what the data obtained means, and guidance on how to interpret the data received. 


\subsubsection{Non-Standard Monitoring Strategies}

There are a number of strategies available to monitor and quantify the performance of attenuation and remediation systems that employ natural processes. Many of these strategies are based on measurements of contaminant concentrations, degradation products, geochemical conditions, and surrogate/indicator parameters in monitoring wells. The fundamental goal of monitoring MNA/EPR is to show that the plume is stable or shrinking and to document the contaminant loading and the natural attenuation capacity of the system. Given this more generally stated objective, a variety of monitoring strategies that are not based on monitoring wells may show promise. These include integrating and volumetric methods such as geophysical techniques, remote sensing and macrobiological indicators. Some types of sensors (e.g., open path infrared sepctroscopy, distributed fiber optic sensing, and the like) and modified monitoring well geometries (e.g., horizontal or angle wells) also show potential and are addressed in the sensor line of inquiry and the monitoring system configuration line of inquiry, respectively.

The most significant cost and commitment in MNA/EPR is long term monitoring. Nonstandard monitoring, if successfully developed to convincingly document performance could reduce the type and number of measurements needed from a monitoring well network. Additionally, such measures have the potential to be more convincing to stakeholders because they may be more robust to heterogeneity that point measurements under some circumstances and may provide data that more directly answers the basic question - is the system keeping humans and the environment safe from exposure?

The three general categories of nonstandard monitoring considered were geophysics, various types of landscape-scale biomonitoring, and remote sensing. Each of the concepts was briefly examined in terms of the available literature and potential for contribution to MNA/EPR. The evaluation suggested that nonstandard monitoring methods that are not based on monitoring wells - have significant limitations. Except under specific circumstances, it is unlikely that a system based only on nonstandard methods would provide adequate monitoring of an MNA/EPR process. Nonetheless, various specific methods and approaches showed significant promise to reduce the quantity of monitoring well data necessary for documentation and to provide strong data to document overall performance. Particular nonstandard monitoring concepts that show promise are listed below. Those concepts that have significant potential for inclusion into protocols in the near term (circa three years) are highlighted, as well as those that have future promise and may be appropriate for additional basic science investments. The promising near-term activities have the most support in terms of scientific literature and can be matched with specific goals in a MNA/EPR monitoring paradigm. Many of the promising near term activities are cross-over 
applications from other disciplines, such as "precision agriculture." The promising longer-term activities are generally those concepts that do not have sufficient scientific support at this time but that would be useful if current basic science research leads to a favorable result.

\section{Most Promising Near-Term Science and Technology Targets}

- Remote Sensing of evapotranspiration and similar parameters -- for systems where water balance and controls are central to performance

- Monitoring of integrating or bioconcentrating indicator species such as honeybees, mollusks, or fish - for systems where information on low concentrations and patchy releases would be useful.

- Monitoring of general ecosystem structure and function (using diversity indices, trophic level indices, and the like)- to efficiently document minimal ecological impacts.

- Geophysical techniques - particularly electrical methods using a permanent electrode array -- for cases where changes in conductivity are related to loading or the maintenance of appropriate chemical conditions for attenuation processes to occur.

Most Promising-Long Term Science and Technology Targets:

- Multispectral and hyperspectral imagery correlated to contaminant exposure and impacts.

\subsubsection{Fate and Transport Modeling - Directions and Opportunities to Support and Accelerate Natural Attenuation and Remediation}

Models are tools that can be used to quantify attenuation and transport processes and thereby estimate the migration of contaminants. A modeling analysis provides this estimate within the context of the conceptual model for the site and with uncertainties defined by 1) the quality and quantity of data to describe the site attenuation and transport processes, and 2) the limitations of the model configuration and analysis technique. The estimates produced by models can be used to predict the future fate of contaminants and to help interpret characterization and monitoring data for the site. Numerical models, in particular, have the computational ability to estimate the interaction of multiple processes temporally and spatially for scenarios that would be difficult to assess with analytical methods. Modeling needs to be designed and used carefully, however, because some models (e.g., detailed numerical models) are data intensive and may not sufficiently reduce uncertainty or improve decisions to justify the required cost and time -- especially at relatively simple sites. Nonetheless, appropriate modeling analysis and predictions can be valuable, along with other site information, in making timely decisions regarding implementing MNA/EPR remedies or for planning monitoring activities. A key function of the predictive capability of 
models is to estimate whether the remedy will meet the remediation goals when this cannot typically be determined directly with field data.

In the simplest sense, modeling analysis estimates the contaminant loading and the natural attenuation capacity of a system. Models serve several other important functions. Models provide a documented approach to link characterization and monitoring data to estimates of performance and provide a tool to examine potential system responses to selected conditions such as transient hydrologic conditions, variations in attenuation processes, and use of remediation techniques. The analysis and predictive functions of modeling are important within the context of applying MNA to 1) help analyze the relative importance of different attenuation and transport processes within a plume, 2) provide timely decision support, for instance, when there is insufficient temporal monitoring data available, 3) evaluate MNA as a remedy to replace existing remedies (e.g., P\&T) that have perturbed the plume such that data to establish whether the plume is stable will not be available for a long time, 4) evaluate combinations of other remedial actions (e.g., Enhanced Passive Remediation (EPR)) and MNA, and 5) help interpret monitoring data for transient plumes. For some sites, use of modeling can have a significant role in establishing the technical basis for evaluating MNA and related EPR as remedies for plumes or to transition from other remedies to $\mathrm{MNA} / \mathrm{EPR}$.

The type of model applied at a specific site is dependent on the site conditions and the intended use of the model. Two basic levels of models are available that are relevant to MNA modeling. Analytical models are capable of solving the general transport equation with specific limitations. Three-dimensional multi-species reactive transport numerical models discretize the transport equation and iteratively solve it within a defined numerical domain. Numerical models allow for more detailed configuration of the model domain to more closely match site features and, therefore, have advantages for some sites. Selection of the appropriate model for a specific site is dependent on the site conditions and configuration-related differences between analytical models and numerical models. Table 1 provides a brief overview of considerations for selecting the primary type of modeling analysis based on site properties, in particular based on whether the geochemistry and hydrology of the site readily supports a relatively simple description of attenuation and transport processes or the geochemistry and hydrology is complex. Other considerations for model selection are discussed below. 
Table 1. Considerations for Selecting Modeling Approach Based on Site Properties KEY:

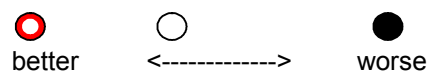

\begin{tabular}{|c|c|c|c|c|c|}
\hline \multirow[b]{2}{*}{ Modeling Approach ${ }^{1}$} & \multicolumn{2}{|c|}{$\begin{array}{l}\text { Sites with supportive geochemical/hydrologic } \\
\text { conditions }\end{array}$} & \multicolumn{3}{|c|}{ Sites with hydrologic \&/or geochemical complexity/challenges } \\
\hline & $\begin{array}{c}\text { Simple site with stable } \\
\text { or shrinking plume }\end{array}$ & $\begin{array}{l}\text { Plume stability \& } \\
\text { geochemical } \\
\text { footprints uncertain }\end{array}$ & $\begin{array}{c}\text { Documented plume } \\
\text { growth or outcrop -- } \\
\text { may be stable in the } \\
\text { future }\end{array}$ & $\begin{array}{c}\text { Geochemical } \\
\text { conditions uncertain } \\
\text { and/or complex } \\
\text { hydrologic conditions }\end{array}$ & $\begin{array}{c}\text { Attenuation process } \\
\text { enhancement } \\
\text { evaluation }\end{array}$ \\
\hline $\begin{array}{l}\text { Conceptual Model - } \\
\text { Identify contributing } \\
\text { processes and the } \\
\text { active zones within a } \\
\text { plume }\end{array}$ & O & $\bigcirc$ & 3 & 3 & \\
\hline $\begin{array}{l}\text { Conceptual Model plus } \\
\text { Analytical Model or } \\
\text { Mass Balance } \\
\text { Calculation }\end{array}$ & $\bigcirc$ & 0 & $\bigcirc$ & $\bigcirc$ & $\bigcirc$ \\
\hline $\begin{array}{l}\text { Conceptual Model, } \\
\text { possible analytical } \\
\text { model and numerical } \\
\text { model }\end{array}$ & 2 & $\bigcirc$ & 0 & 0 & O \\
\hline
\end{tabular}

${ }^{1}$ Underlining indicates the primary modeling analysis considered for the table row.

${ }^{2}$ Numerical modeling is not necessarily preferred because costs may not be justifiable for the offsetting benefits in terms of uncertainty reduction, monitoring optimization, etc. However, numerical models may be selected if it is necessary to provide better estimates of timeframes and better assurance of meeting certain types of remediation goals (e.g., concentration targets) than can be obtained with analytical modeling.

${ }^{3}$ Conceptual models are good to use for planning and site management, but may not be suited as primary support for decision making at complex sites or sites that have high uncertainty because conceptual models do not allow testing of uncertainty and parameter sensitivity and do not strongly support a detailed evaluation of enhancements.

Analytical models such as BIOCHLOR (Aziz et al., 1999) have been established specifically for use in modeling MNA. For analytical models, the solution technique typically requires assumptions of uniform hydraulic properties throughout the domain, uniform steady-state groundwater flow (in some case limited to onedimensional advection), simple boundary conditions, simple source geometry, firstorder contaminant transformation with rates constant within a defined area (in some cases for a single decay pathway), and uniform linear equilibrium partitioning. Analytical models can be useful in providing estimates of contaminant migration for plumes where these assumptions can be technically supported based on the site conditions.

Numerical models may be needed when site conditions cannot be described under the simplified flow, reaction, or adsorption process assumptions required for use of analytical models. The groundwater flow system at a site may not be uniform because of a complex distribution of hydraulic conductivity, complex recharge/discharge elements, or transient flow conditions. Sources distributed in 
multiple locations, multiple contaminant species with multiple reaction pathways, and multiple oxidation/reduction conditions within the plume area cause complexities in modeling the reaction processes at a site. In some cases, assumption of linear equilibrium sorption is not appropriate depending on the nature of the contaminant and the aquifer solids. For site conditions that include any or all of these complexities, numerical models are more appropriate than analytical models. For instance, the publicly-available Reactive Transport in 3-Dimensions (RT3D) code (Clement et al., 1998; Clement, 1997) provides a framework to solve for reactive transport under these more complex conditions using the MODFLOW code (McDonald and Harbaugh, 1988) to determine groundwater flow.

Similar to analytical models, numerical models have limitations in how they can be configured to match site conditions. Equations cannot describe all of the nuances for each term within the transport equation. That is, numerical models cannot exactly reproduce reality. Compared to analytical models, numerical models can be configured to more closely match the site conditions and processes. There are also limitations in the type and quality/quantity of data that are available at any site to develop the coefficients necessary in the equations for the numerical model. Given these limitations, with appropriate interpretation of the results, numerical models can be very useful in the roles described above.

Interpretation of model results is dependent on the context of how the modeling is being applied. Using a model, multiple simulations can be conducted with variations in the input parameters. By comparing the results of these simulations, the relative importance of specific processes can be assessed. This modeling approach can be implemented with simple single parameter variation or using a statistical approach such as the Monte Carlo process. Predictive simulations are used to estimate future plume migration under the selected remediation scenario (e.g., MNA/EPR) and thereby assess the ability of the remedy to meet remediation goals. In some cases, it is appropriate to select specific simulation scenarios and use the calibrated model to assess whether remediation goals will be met under these selected conditions. Alternatively, the modeling approach may include conducting a statistical series of simulations (e.g., Monte Carlo analysis) to predict the probability for future contaminant distributions.

The results of multiple simulations can be interpreted to assess the uncertainty of the modeling results; that is, assessing how variations in input parameter data impact the model results. This uncertainty is important to consider in evaluating whether additional characterization for model input parameters is needed or to evaluate the technical risk of a decision based on the model results for the given level of information about the model inputs and the model configuration/calibration.

Based on the information in the modeling line of inquiry, there are several elements of modeling that merit attention for improving how models are used to support an 
MNA remedy. In the near term, improvement in these elements may enable improved quantification of attenuation processes, better integration of modeling within the MNA evaluation and implementation process, and expanded application of modeling to support better strategies for characterization and monitoring associated with MNA. In summary, these important elements are:

- Define when to use numerical modeling and how to integrate modeling with other elements of MNA evaluation and implementation.

- Improve the description and understanding of the modeling process for MNA.

- For example, clarify methods to address uncertainty in model inputs and in model interpretation.

- Address numerical model complexity.

$\bigcirc \quad$ Improve the description and understanding of the modeling process for MNA.

- For use of analytical models, address specific limitations of current analytical models.

- Improve BIOCHLOR with expanded reaction forms and variable retardation.

- Improve specific process descriptions for numerical models.

- Variable sorption phenomena (e.g., a migration resistant fraction)

- Sustainability of reactions

- Abiotic heterogeneous reaction descriptions

- Substrate and electron acceptor correlations to dechlorination reactions

- Integrate modeling with characterization and monitoring.

- Develop new characterization/monitoring techniques that are integrated with model input requirements or interpreted using modeling results.

- For instance, combine modeling with implementation of techniques such as flux monitoring or identify measurement techniques for process parameters such as quantifying time-variant sorption phenomena for a specific site.

\subsubsection{Sensors}

The US DOE is seeking methods to determine and prioritize sensor needs for MNA. Current guidelines (e.g., US EPA, 1998) have specified a list of parameters that should be quantified for determining the viability and continuance of MNA at a site. This list includes concentration (and historical concentration) of the contaminant and corresponding degradation products as well as parameters consistent with conditions allowing the degradation process (e.g., $\mathrm{O}_{2}$ concentration, redox, appropriate bacteria, etc.). Sensing and monitoring for MNA must be able to effectively prove the abatement of the contaminant yet the cost cannot be greater than the cost for baseline active treatment such as pump and treat. These requirements clearly call for modifications of current monitoring techniques and a creative balance between 
baseline (or modified baseline) and innovative technologies that efficiently contribute to the refinement and verification of the site conceptual model.

The first step in increasing the cost effectiveness of sensing and monitoring is recognizing that different phases of cleanup require different technology attributes. Four different phases of characterization and monitoring with respect to MNA are defined earlier in this document as screening characterization, decision characterization, process monitoring, and system performance monitoring. For MNA the clearest difference in requirements is that between process monitoring where validation or proof of the process is the objective, and system performance monitoring where validation of performance (or continuity of the process) is the objective. For these two phases, sensors are needed that are specific to priority MNA parameters as well as conventional contaminant concentration sensing. Process monitoring confirms the degradation processes of the conceptual model and establishes a data set that verifies the process is continuing for comparison of the status of the subsurface system, as well as identifying system-specific measurement parameters that can easily and inexpensively be used for system performance monitoring. System performance monitoring requires comparable measurement methods that will extend over long time periods that indicate or confirm that the processes identified in the conceptual model are continuing to occur or have stopped.

\section{Opportunities for Innovative Technologies or Strategies}

Based on the results of the survey included in this report the primary parameter measured by practitioners of MNA continues to be concentration of the contaminants and their degradation products although other parameters such as dissolved oxygen were also deemed useful. Many other parameters that are generally considered to be important aspects and indicators of MNA are not routinely measured. This may be interpreted primarily as a result of a lack of understanding of how these parameters are important to the MNA process or that the additional costs (either monetary or level of complexity) for measuring these parameters did not provide a commensurate benefit in understanding of the system. Hydraulic parameters were not addressed in the survey although these are undoubtedly important for understanding the behavior of the system particularly in cases where an understanding of the stability of the plume (i.e., shrinking, expanding, or stable) is vital. Confidence that MNA processes are occurring over the bulk of the subsurface zone of interest suggests the usefulness of volumetric knowledge of the site achieved either by multiple or integrated sensing/sampling points or measurements of flux and understanding of the multiplicity of flux paths. The flux of the contaminant is generally agreed to be the most important aspect of contaminant interaction yet the flux is not generally regulated nor routinely measured for chemical contaminants. Some potentially innovative technologies and techniques for long term sensing and sampling and potential areas for future investment are listed below (and expanded as appropriate in Section 7.0): 
- IR spectroscopy methods

- Other optical spectroscopy techniques (e.g., oxygen sensor using optical fluorescence)

- Flux Measurements

- Threshold and binary sensing

- FLUTe based samplers or indicators

- Heterogeneous Flow Measurements

- In situ sparging/phase transfer methods

- Fiber optic sensors (e.g., evanescent wave/cladding sensors)

- Inexpensive rapid access techniques coupled with sensors or inexpensive samplers

- Controlling/Master Variable Sensors (e.g., Pressure, Temp, pH, Eh, $\mathrm{O}_{2}$, flow, moisture)

- Open Path FTIR and other non point monitoring

\section{Other Aspects of Sensing and Monitoring}

SpatioTemporal Frequency. There is rarely enough thought given to the frequency requirements of subsurface measurements both with respect to time and space. The frequency (spatio-temporal) of the measurement should be modeled after a modified Nyquist Theorem ${ }^{1}$, (i.e., enough measurements to prevent aliasing) where sampling should occur at more than twice the rate of the fastest frequency of interest of the measurement (Nyquist, 1928; Shannon 1949). This may or may not be the apparent fundamental frequency and the frequency of interest will certainly change.

Concentrations in the subsurface can be affected by many processes. For example, small amplitude, relatively high frequency changes in concentration occurring diurnally as a result of solar temperature cycling may be of no interest in a long term monitoring objective. Therefore, a sampling or sensing schedule should not be designed for this frequency. In another example, large amplitude concentration changes related to pressure cycling from 4 day weather fronts may be of interest at first then of little interest once the cycle is understood and can be accounted for. Nutrient depletion occurring on a monthly or yearly (or other) cycle may be the frequency of interest for a system and set the temporal measurement resolution parameter, though the rate of degradation will be the most likely frequency of interest. Rates of degradation for MNA processes may occur on the order of several years, so a well-behaved system with few other processes that affect concentration would need very infrequent monitoring.

\footnotetext{
${ }^{1}$ The Nyquist theorem and aliasing are simple-powerful techniques to assist in determining sampling frequency and interval. While used in the past, expanded future application of these and similar methods are promising.
} 
The idealized cyclical processes in this example are extremely simplified, though cyclical trends are often observed in natural systems. For processes with a cycle that is difficult to recognize, event sensing may be a more practical method for accurately monitoring a system. Event sensing (generally at a lower data quality level e.g., threshold or other binary or limited dynamic range) can be used for triggering a more detailed sampling event. Although this implies much higher frequency data, the analytical quality of the data is much less stringent.

Like selection of an appropriate temporal frequency, spatial measurement resolution must be selected based on a relevant scale. High-spatial resolution data are desirable in the initial phases of understanding a system (validation of process) but may be less necessary for longer term monitoring. High spatial resolution is also tempered by the cost of acquiring the data. High axial resolution data are generally possible during drilling or penetration because the access system makes continuous progress through the subsurface; however, resolution away from the penetration axis is limited by the budget for number of holes. Luckily in many sedimentary systems, significant changes occur more frequently at the vertical scale than the horizontal scale. Conceptual, analytic and numerical models should be used interactively with sensing strategies to tune sensing and refine the model.

Point Sampling vs Volume Probing Methods. Volume probing (usually manifest as an integration of points as in longer screened wells) may be the most useful way to effectively monitor for threshold exceedances or to verify the average behavior occurring in microenvironments. Point samples capture the extreme values in a plume and a high density of points should certainly be used to characterize it. However, smaller numbers of point samples can misrepresent the bulk behavior of a plume particularly in a long term monitoring application. Larger volume or integrated samples may be more effective for this monitoring objective. The danger in larger volume measurements is the addition of too much noise to accurately measure the important signal. The volume should be well matched to the zone of interest to reduce noise and sensing or analysis methods must be sensitive enough to accommodate some dilution. Careful sizing of screen lengths can be an effective way to accomplish this.

In Situ or Ex Situ Sensing. Ideally, in situ sensing is preferable to ex situ methods primarily because it more accurately represents the processes occurring in the subsurface (the extraction process by definition severs interactive processes) and the in situ process by nature requires a minimum of human intervention. Unfortunately there are very few in situ technologies that are suitable for long term monitoring. Ex situ sensing is therefore generally, used such as when a sample is extracted from the subsurface and analyzed either in the laboratory or in the field. If the sample is not modified (with respect to the parameter of interest) ex situ methods are satisfactory for site monitoring. If the sample can be extracted automatically and analyzed in the 
field without human intervention at a reasonable cost, the method approaches the ideal.

\subsubsection{Monitoring System Configuration}

The configuration of monitoring systems incorporates the short and long term monitoring phases for MNA. The goals of the process monitoring (short term) configuration are to monitor the contaminant loading versus the attenuation capacity of the system, validate the conceptual model, and design the long-term system performance monitoring configuration. The monitoring network (monitoring well/point geometry) is designed to effectively define the spatial and temporal physical and chemical characteristics of the 3-dimensional plume and aquifer. The process monitoring phase will likely follow existing federal protocols that rely on concentration and other MNA parameter measurements from monitoring wells. The anticipated results from the process monitoring are 1) confidence that MNA is the appropriate remedial action for the site, 2) identification of leading indicator values for long-term monitoring, 3) long-term monitoring frequency, and 4) guidance for addressing system changes or failure.

The final phase of monitoring, system performance monitoring, represents the most mature stage of monitoring. Attenuation mechanisms have been identified, baseline data exists and the conceptual model has been validated. The goals of this stage of monitoring are to 1) monitor remediation performance, 2) document response to system changes, and 3) confirm baseline mechanisms and rates. During this phase, the emphasis is to reduce costs and use leading indicator parameters that monitor the overall health of the MNA process. The monitoring configuration minimizes the number of measurements while maintaining confidence that MNA is meeting the remedial goals. The monitoring methods incorporate flexible, low cost, passive, low maintenance and robust indicators for the MNA using multiple lines of evidence. The monitoring network (monitoring well/point geometry, remote sensing, etc.) is designed to effectively obtain leading indicator data to analyze the overall system and may or may not use the existing monitoring network. System performance monitoring for the long-term will depend on developing wholly new verification paradigms to allow monitoring for decades or centuries. Large-scale methods (remote sensing, geophysics) and integrating methods (flux or release measurements), among others, appear promising during this phase. 


\subsection{Identification of Short Term and Long Term Science and Technology Targets}

The detailed evaluation within each of the lines of inquiry was used to identify targets that show particular promise to facilitate the implementation of MNA/EPR. Each of the lines of inquiry generated from five to ten promising items that were compiled into a master list for prioritization. The entire sequence proved to be quite effective. The initial evaluation in the lines of inquiry reduced the potential list of topics to research from an essentially unlimited set to a short list of 47 items. The types of screening that occurred in this first phase included: a) the potential value and technical risk, and $b$ ) the need for the work in the context of other investments in MNA research (SERDP, ESTCP, EMSP, etc. - see acronym list for definitions). The next step of the process ranked all of the promising technologies/concepts that were raised up from the lines of inquiry into different categories and priorities. The ultimate result was a strategic list that will serve as the roadmap to guide the US DOE MNA/EPR Alternative Project and maximize the project's potential to be efficient and to provide an impact to accelerate use of MNA/EPR at US DOE sites.

The team emphasized four major factors in the evaluation: 1) the item should support the framework of attenuation capacity and loading, 2) the item should support the transition of a site into a protective cost effective system performance monitoring state, 3 ) the item should move toward direct and integrated measures of processes (rather than indirect measures), and 4) enhancements for EPR should be naturally sustainable. By emphasizing this short list of criteria, the team felt the ultimate portfolio would consist of synergistic items that will move MNA/EPR strongly in the desired direction.

In the evaluation process, it was clear that some of the items being ranked were policy issues and some were technical and implementation issues (and some overlap these areas). The team made an assessment of whether an item was predominantly policy, or predominantly technical or predominantly implementation (i.e., technology exists and may be useful without much further development) and noted that in the evaluation. For each technology the pros and cons were tabulated, the approximate cost-range, the R\&D timeframe and development timeline, and an overall ranking were identified. These categories were important because the US DOE MNA/EPR Alternative Project has a limited budget and scope and a strategically blended portfolio of targets is needed so that the research will have the best chance of providing value. Thus, excessive costs for any one item (that would reduce the diversity of the portfolio) or long timeframes (greater than the proposed project duration) decreased the overall priority for this project. This does not imply that the activities are not important. The long-term targets are more suited to basic science and ongoing research programs and they are separately tabulated to assist in developing future research calls in those programs. The policy priorities, which are equally important, have been separately tabulated and will be provided to the regulatory partners in the project, including ITRC and US EPA. Although ITRC does not set policy, input from this organization on the items identified as policy priorities may provide useful insight for this project. In the end, the process identified 16 high priority technical targets for this project, as presented in Table 2. All of these targets were considered "ready for MNA deployment" or 
"supportive of MNA protocol development in the next three years." The various high priority items were supportive and integrated, as desired. The team identified 4 high priority policy targets, Table 3, and 6 high priority basic science (long term research) target categories, Table 4.

The overall prioritization, including the detailed discussion, is in the large tables in the appendix. The high priority research and policy targets are presented below in tables that provide only the titles and approximate cost range. 
Table 2. High Priority Technical and Implementation Targets Recommended for US DOE MNA/EPR Alternative Project

\begin{tabular}{|c|c|}
\hline & Cost \\
\hline \multicolumn{2}{|l|}{ Direct measures of Attenuation Mechanisms } \\
\hline $\begin{array}{l}\text { a) Develop advanced bioassessment tools for determining ongoing and } \\
\text { potential microbial processes at different sites. } \\
\text { - includes reductive processes (Dehalococcoides etc.) } \\
\text { - new methods to quantify growth. } \\
\text { - gene sequences used to develop molecular probes for micro arrays and } \\
\text { quantitative PCR. } \\
\text { - additional key functional genes for use in Microarrays } \\
\text { - additional key functional genes for use in Microarrays } \\
\text { - genes whose expression correlates with specific environmental conditions } \\
\text { - DNA microarrays used to simultaneously monitor the expression of many } \\
\text { genes }\end{array}$ & $\$ \$$ \\
\hline $\begin{array}{l}\text { b) Develop field scale correlations between species, functional genes, and } \\
\text { degradation rate and potential } \\
\text { - Laboratory and field tests to develop and correlate parameters } \\
\text { - Assess factors that would bias the application of these molecular tools }\end{array}$ & $\$ \$$ \\
\hline $\begin{array}{l}\text { c) Conduct further research on oxidative and reductive processes (several } \\
\text { specific sub-needs listed) }\end{array}$ & $\$ \$$ \\
\hline $\begin{array}{l}\text { d) Develop direct measures and sensors for abiotic attenuation } \\
\text { mechanisms. e.g. resistive fraction; develop method to measure; results could } \\
\text { be used to establish capacity as well as provide concentration at which to } \\
\text { transition from active treatments }\end{array}$ & $\$$ \\
\hline \multicolumn{2}{|l|}{ Strategies to Support Characterization and Monitoring } \\
\hline $\begin{array}{l}\text { e) Develop a scenarios based framework to support characterization } \\
\text { monitoring and modeling decisionmaking } \\
\text { - Taxonomic key to help define multiple lines of evidence. }\end{array}$ & $\$$ \\
\hline $\begin{array}{l}\text { f) Develop specific alternative monitoring configurations to collect data at } \\
\text { substantial cost savings during system performance monitoring stage } \\
\text { - Examples include horizontal wells to replace several vertical wells. }\end{array}$ & $\$$ \\
\hline $\begin{array}{l}\text { g) Improve integration of modeling into MNA evaluation and } \\
\text { implementation process }\end{array}$ & $\$$ \\
\hline $\begin{array}{l}\text { h) Improve reaction forms and include variable linear equilibrium } \\
\text { partitioning coefficients within analytical models }\end{array}$ & $\$$ \\
\hline \multicolumn{2}{|l|}{ Tools to Support Characterization and Monitoring } \\
\hline $\begin{array}{l}\text { i) Develop threshold and binary monitoring devices and strategies for } \\
\text { long-term monitoring }\end{array}$ & $\$$ \\
\hline
\end{tabular}




\begin{tabular}{|c|c|}
\hline & Cost \\
\hline $\begin{array}{l}\text { j) Remote sensing of a parameter(s) that can be related to system } \\
\text { performance } \\
\text { - remote sensing of evapotranspiration and similar parameters }\end{array}$ & $\$$ \\
\hline $\begin{array}{l}\text { k) Develop spectroscopy methods (IR and other optical methods) to } \\
\text { measure critical MNA parameters } \\
\text { - oxygen sensor using optical fluorescence }\end{array}$ & $\$$ \\
\hline l) Develop improved dissolved oxygen measurement approaches & $\$$ \\
\hline $\begin{array}{l}\text { m) Develop methods to measure flux more directly than traditional } \\
\text { methods (groundwater and contaminant flux). }\end{array}$ & $\$$ to $\$ \$$ \\
\hline n) Samplers that integrate over distances and volumes & $\$$ \\
\hline \multicolumn{2}{|l|}{ Use of passive or semipassive enhancements } \\
\hline $\begin{array}{l}\text { o) Microbiology - Bioaugmentation } \\
\text { - Anaerobic systems (with Dehalococcoides) } \\
\text { - Aerobic systems (with JS666) }\end{array}$ & $\$ \$$ \\
\hline $\begin{array}{l}\text { p) Modifying Large-scale Hydrology } \\
\text { - Bypass of upgradient water } \\
\text { - Decrease infiltration throughout plume } \\
\text { using increase runoff } \\
\text { using increased evapotranspiration } \\
\text { - Decrease mass discharge from source } \\
\text { - Bypass of competing electron acceptors }\end{array}$ & $\$$ to $\$ \$$ \\
\hline
\end{tabular}

Key:

Approximate cost for initial research effort

$\$=\leq \$ 100 \mathrm{~K}, \$ \$=>\$ 100 \mathrm{~K}-<\$ 500 \mathrm{~K}, \$ \$ \$=>\$ 500 \mathrm{~K}$ 
Table 3. High Priority Policy Targets Recommended for US DOE MNA/EPR Alternative Project

\section{Policy Targets}

Develop decision process (based on science) to transition from active to more passive treatment

Develop concurrence with the stages of characterization and monitoring and assure alignment with CERCLA/RCRA etc, decision-making process.

Develop acceptance of central concept defining MNA/EPR - Balancing Attenuation Capacity and Loading

Acceptance of framework for selecting the analytes and interpreting the results.

A decision tree or scenario based structure would be examples 
Table 4. High Priority Technical Targets for consideration by basic science research programs such as EMSP and SERDP

\begin{tabular}{|l|}
\hline \multicolumn{1}{|c|}{ Long Term and Basic Science Targets } \\
\hline Phytoremediation (all types) \\
- Baseline studies \\
- Fertilization studies \\
- New species studies \\
- Genetic engineering and selection studies \\
\hline Biological and Ecosystem Techniques \\
- Monitoring of indicator species such as honeybees, mollusks, or fish - for systems \\
where information on low concentrations and patchy releases would be useful. \\
- Monitoring of general ecosystem structure and function (using diversity indices, \\
trophic level indices, and the like) - to efficiently document minimal ecological \\
impacts \\
\hline Geophysical Techniques \\
Such as electrical methods using a permanent electrode array -- for cases where \\
changes in conductivity are related to loading or the maintenance of appropriate \\
chemical conditions for attenuation processes to occur. \\
\hline Remote Sensing. \\
Such as Multispectral and hyperspectral imagery correlated to contaminant exposure \\
and impacts \\
\hline Direct Measures of Sustainability and Risk \\
- Link to current SERDP activities examining sustainability; \\
- Look at methods to directly measure risk and potentially system capacity. \\
e.g. DNA on a chip \\
\hline Measurement at a variety of scales \\
- Such as Heterogeneous Flow Measurements \\
- Open Path FTIR and other non point monitoring, etc. \\
\hline
\end{tabular}




\subsection{References}

\subsection{Cited References}

American Society of Testing and Materials (ASTM), 1998, Guide for Remediation by Natural Attenuation at Petroleum Release Sites: ASTM Philadelphia, PA.

Anderson, T. A. and Walton, B. T. 1995. Comparative fate of ${ }^{14} \mathrm{C}$-trichloroethylene in the root zone of plants from a former solvent disposal site. Environ. Toxicol. Chem., 14: 2041-2047.

Aziz, C.E., Newell, C.J., Gonzales, J.R., Haas, P., Clement, T.P., and Sun, Y. 1999. BIOCHLOR Natural attenuation decision support system v1.0. User's Manual, U.S. EPA Report, EPA 600/R$00 / 008$.

Clement, T.P. 1997. RT3D - A Modular Computer Code for Simulating Reactive Multi-Species Transport in 3-Dimensional Groundwater Aquifers. Pacific Northwest National Laboratory, Richland, WA. PNNL-11720.

Clement, T.P., Sun, Y., Hooker, B.S., and Petersen, J.N. 1998. Modeling Multi-Species Reactive Transport in Groundwater Aquifers. Groundwater Monitoring \& Remediation Journal, 18(2): 7992.

Dickmann, D.I., and Stuart, K.W. 1983. The Culture of Poplars in Eastern North America. Hickory Hollow Associates, Dansville, MI.

Dickmann, D.I., Nguyen, P.V., and Pregitzer, K.S. 1996. Effects of irrigation and coppicing on aboveground growth, physiology, and fine-root dynamics of two field-grown hybrid poplar. For. Ecol. and Manage, 80: 163-174.

Doty, S.L., Shang, T.Q., Wilson, A.M. Tangen, J., Westergreen, A.D., Newman, L.A., Strand, S.E. and Gordon, M.P. 2000. Enhanced metabolism of halogenated hydrocarbons in transgenic plants containing mammalian cytochrome P450 2E1. Proc. Natl. Acad. Sci., 97: 6287-6291.

Hansen, E.A. 1993. Soil carbon sequestration beneath hybrid poplar plantations in the north central United States. Biomass and Bioenergy, 5: 431-436.

Inter-State Technology Regulatory Council (ITRC), reprinted September 1999. Natural Attenuation of Chlorinated Solvents in Groundwater: Principles and Practices.

McDonald, M.G. and Harbaugh, A.W. 1988. A Modular Three-Dimensional Finite-Difference GroundWater Flow Model. Techniques of Water-Resources Investigations 06-A1, U.S. Geological Survey Open-File Report 83-875.

National Research Council. 1993. In Situ Bioremediation, When Does it Work?: National Academy Press, Washington, D.C., 207 p.

National Reseach Council, 2001. Natural Attenuation for Groundwater Remediation, National Research Council, Committee on Intrinsic Remediation, National Academy Press, Washington, D.C. 
Newell, C.J., Aziz, C.E., and Cox, G.A. 2001a. "Novel Method to Enhance Chlorinated Solvent Biodegradation by the Use of Barriers," 2001 Int. Containment \& Rem. Technology Conf., June 10-13, 2001, Orlando, Florida.

Newell, C.J., Aziz, C.E., Haas, P.E., Hughes, J. B., and Khan, T.A. 2001b. "Two Novel Methods for Enhancing Source Zone Bioremediation: Direct Hydrogen Addition and Electron Acceptor Diversion", Proceedings of the Battelle Sixth International In Situ and On-Site. Bioremediation Symposium, June 4-7, 2001. San Diego, CA, Battelle Press, Columbus, Ohio, In Press.

Newell, C.J., C.E. Aziz, and G.A. Cox, 2003. "Enhanced Anaerobic Treatment Zones in Groundwater," U.S. Patent 6,562,235; May 13, 2003.

Nyquist, H. 1928. Certain topics in telegraph transmission theory, Trans. AIEE, 47: 617-644.

Shannon, C.E. 1949. Communication in the presence of noise, Proc. Institute of Radio Engineers, 37(1): $10-21$.

Sophocleous, M.A. 2002. Interactions between groundwater and surface water: the state of the science. Hydrogeology Journal, 10: 52-67.

Stettler, R.F., Koster, R., and Steenackers, V. 1980. Interspecific crossability studies in poplars. Theor. Appl. Genet., $58: 273-282$.

Tschaplinski, T.J., and Blake, T.J. 1989a. Correlation between early root production, carbohydrate metabolism, and biomass production in hybrid poplar. Can. J. Bot., 67: 2168-2174.

Tschaplinski, T.J., and Blake, T.J. 1989b. Water stress tolerance and late-season organic solute accumulation in hybrid poplar. Can. J. Bot., 67: 1681-1689.

Tschaplinski, T.J., and Blake, T.J. 1994. Carbohydrate mobilization following shoot defoliation and decapitation in hybrid poplar. Tree Physiol. 14: 141-151.

Tschaplinski, T.J., and Tuskan, G.A. 1994. Water-stress tolerance of black cottonwood and eastern cottonwood clones and four of their hybrid progeny. II. Metabolites and inorganic ions that constitute osmotic adjustment. Can. J. For. Res., 24: 681-687.

Tuskan, G.A. 1998. Short-rotation woody crop supply systems: We do we know and what do we need to know? Biomass and Bioenergy, 14: 307-315.

US Department of Energy (US DOE) 2002b. Alternative Project Plan for Technology Development, Accelerated Risk Reduction Through Innovative Remediation of Chlorinated Ethenes Using Monitored Natural Attenuation. US DOE Office of Environmental Management.

US Environmental Protection Agency (US EPA) 1998. Technical protocol for evaluating natural attenuation of chlorinated solvents in ground water, EPA/600/R-98/128: Office of Research and Development, U.S. Environmental Protection Agency, Washington, D.C., p. 232.

US Environmental Protection Agency (US EPA) 1999a. Use of Monitored Natural Attenuation at Superfund, RCRA Corrective Action, and Underground Storage Tank Sites, Final, Office of Solid Waste and Emergency Response. April 21. Directive Number 9200.4-17P. 
US Environmental Protection Agency (US EPA), 1999b, Use of Monitored Natural Attenuation at Superfund, RCRA Corrective Action, and Underground Storage Tank Sites, Draft Interim Final :U.S. EPA Office of Solid Waste and Emergency Response Directive 9200.4-17 (http://www.epa.gov/OUST/directiv/9200417z.htm)

US Environmental Protection Agency (US EPA) 2000. Proceedings of the Ground-water/surface-water interactions workshop. EPA/542/R-00/007.

Westinghouse Savannah River Company, 2003. Scientific Basis for Monitored Natural Attenuation and Enhanced Passive Remediation for Chlorinated Solvents - DOE Alternatie Project for Technology Acceleration Implementation Plan. WSRC-RP-2003-00286. Westinghouse Savannah River Company, Aiken, SC

Wiedemeier, T.H., Wilson, J.T., Kampbell, D.H., Miller, R.N., and Hansen, J.E., 1995, Technical protocol for implementing intrinsic remediation with long-term monitoring for natural attenuation of fuel contamination dissolved in groundwater: US Air Force Center for Environmental Excellence, San Antonio, Texas.

Wiedemeier, T.H., Swanson, M.A., Moutoux, D.E., Wilson, J.T., Kampbell, D.H., Hansen, J.E., and Haas, P., 1996a, Overview of the Technical Protocol for Natural Attenuation of Chlorinated Aliphatic Hydrocarbons under Development for the U.S. Air Force Center for Environmental Excellence: EPA/540/R-96/509, p. 35-59.

Wiedemeier, T.H., Rifai, H.S., Newell, C.J., and Wilson, J.T. 1999. Natural Attenuation of Fuel Hydrocarbons and Chlorinated Solvents, John Wiley and Sons, New York, New York.

Wiedemeier, T.H. and M Barden, 2002, Natural Attenuation for Remediation of Contaminated Sites, National Groundwater Association Course Notes.

\subsection{Comprehensive Reference List from the separate Lines of Inquiry}

Abdel Aal, G.Z. Atekwana, Estella, A., Slater, L.D. and Ulrich, C. 2003. Induced polarization (IP) measurements of solids from an aged contaminated site. SAGEEP 2003 Proceedings, 190-200.

Accardi-Dey, A., and Gschwend, P.M. 2003. Reinterpreting Literature Sorption Data Considering Both Absorption into Organic Carbon and Adsorption onto Black Carbon. Environ. Sci. and Technol. 37: 99-106.

Achtnich, C., Lenke, H., Klaus, U., Spiteller, M., and Knackmuss, H. 2000. Stability of Immobilized TNT Derivatives in Soil as a Function of Nitro Group Reduction. Environ. Sci. and Technol., 34: 36983704.

Acton, D.W. 1990. Enhanced in situ biodegradation of aromatic and chlorinated aliphatic hydrocarbons in anaerobic, leachate-impacted groundwaters: M.Sc. Thesis, University of Waterloo, Waterloo, Ontario.

Adrian, L., et al. 2000. Bacterial dehalorespiration with chlorinated benzenes. Nature, 408: 580-583. 
AFCEE. 1996. Technical Protocol for Evaluating Natural Attenuation of Chlorinated Solvents in Groundwater. Air Force Center for Environmental Excellence, San Antonio, Texas.

Akkermans, A.D.L., et al. 1994. Molecular ecology of microbes: A review of promises, pitfalls, and true progress. FEMS Microbiology Reviews, 15: 185-194.

Alexander, M. 1994. Biodegradation and Bioremediation. San Diego, Academic Press.

Alexander, M. 2000. “Aging, Bioavailability, and Overestimation of Risk from Environmental Pollutants." Environ. Sci and. Technol., 34: 4259.

Amann, R.I., Ludwig, W., and Schleifer, K.-H. 1995. Phylogenetic identification and in situ detection of individual microbial cells without cultivation. Microbiological Reviews. 59: 143-169.

Amann, R. 2000. Who is out there? Microbial aspects of biodiversity. Systematic and Applied Microbiology, 23: 1-8.

Amonette, J.E., Workman, D.J., Kennedy, D.J., Fruchter, J.S., and Gorby, Y.A. 2000. Dechlorination of carbon tetrachloride by Fe (II) associated with goethite. Environ. Sci. and Technol., 34(21): 46064613.

Anderson, R. T. and Lovley, D. R. 1997. Ecology and biogeochemistry of in situ groundwater bioremediation. Adv. Micro. Ecol., J.G. Jones. New York, Plenum Press. 15: 289-350.

Anderson, T. A., Guthrie, E. A., and Walton, B.T. 1993 Bioremediation in the rhizosphere. Environ. Sci. and Technol., 27: 2630-2636.

Anderson, T. A. and Walton, B. T. 1995. Comparative fate of ${ }^{14} \mathrm{C}$-trichloroethylene in the root zone of plants from a former solvent disposal site. Environ. Toxicol. Chem., 14: 2041-2047.

Aochi, Y.O., and Farmer, W.J. 1997. Role of Microstructural Properties in the Time-Dependent Sorption/Desorption Behavior of 1,2-Dichloroethane on Humic Substances. Environ. Sci. and Technol., 31: 2520-2526.

Arp, D.J. 1995. Understanding the diversity of trichloroethene co-oxidations. Current Opinion in Biotechnology, 6: 352-358.

American Society of Testing and Materials (ASTM), 1998, Guide for Remediation by Natural Attenuation at Petroleum Release Sites: ASTM Philadelphia, PA.

Atekwana, Estella A., Atekwana, Elliot A., and Rove, R.S. 2003. Relation between total dissolved solids and bulk conductivity at a hydrocarbon-contaminated acquifer. SAGEEP 2003 Proceedings, 271280 .

Aziz, C.E., Newell, C.J., Gonzales, J.R., Haas, P., Clement, T.P., and Sun, Y. 1999. BIOCHLOR Natural attenuation decision support system v1.0. User's Manual, U.S. EPA Report, EPA 600/R00/008.

Aziz, J. J., Ling, M., Rifai, H. S., Newell, C. J., and Gonzales, J. R. 2003. MAROS: A decision support system for optimizing monitoring plans. Groundwater, 41(3): 355-367.

Baedecker, M.J., and Back, W. 1979 Hydrogeological processes and chemical reactions at a landfill: Groundwater, 17(5): 429-437. 
Bagley, D. M. and Gossett, J. M. 1990. “Tetrachloroethene transformation to trichloroethene and cis-1,2dichloroethene by sulfate-reducing enrichment cultures." Appl. Environ. Microbiol., 56(6): 25112516.

Balkwill, D.L. 1989. Numbers, diversity, and morphological characteristics of aerobic chemoheterotrophic bacteria in deep subsurface sediments from a site in South Carolina, Geomicrobiol., J. 7:33-52.

Ballapragada, B. S., Puhakka, J. A., Stensel, H. D., and Ferguson, J. F. 1995. Development of tetrachloroethene transforming anaerobic cultures from municipal digester sludge. p. 91-97. In R. E. Hinchee, A. Leeson, and L. Semprini (eds.) Bioremediation of chlorinated solvent, Battelle Press, Columbus.

Bankston, J.L., Sola, D.L., Komor, A.T., and Dwyer, D.F.. Degradation of trichloroethylene in wetland microcosms containing broad-leaved cattails and eastern cottonwood. Water Res., 36: 1539-1546.

Barker, J.F., Patrick, G.C., and Major, D. 1987. Natural attenuation of aromatic hydrocarbons in a shallow sand aquifer. Ground Water Monitoring Review, 7: 64-71.

Barrio-Lage, G.A. Parsons, F.Z., Barbitz, R.M., Lorenzo, P.L., and Archer, H. E. 1990. Enhanced anaerobic biodegradation of vinyl chloride in ground water. Environ. Toxicol. Chem., 9: 403-415.

Barrio-Lage, G. A., F. Z. Parsons, R. S. Nassar, and P. A. Lorenzo. 1987. Biotransformation of trichloroethene in a variety of subsurface materials. Environ. Toxicol. Chem., 6: 571-578.

Beja, O., et al. 2000. Bacterial rhodopsin: Evidence for a new type of phototrophy in the sea. Science, 289: 1902-1906.

Bernard, L., et al. 2001. A new approach to determine the genetic diversity of viable and active bacteria in aquatic ecosystems. Cytometry, 43: 314-321.

Bertilsson, S., Cavanaugh, C.M., and Polz, M.F. 2002. Sequencing-independent method to generate oligonucleotide probes targeting a variable region in bacterial 16S rRNA by PCR with detachable primers. Applied and Environmental Microbiology, 68: 6077-6086.

Bicknell, B.R., Imhoff, J.C., Kittle Jr., J.L., Donigian Jr., A.S., and Johanson, R.C. 1997. Hydrological Simulation Program--Fortran: User's manual for version 11: U.S. Environmental Protection Agency, National Exposure Research Laboratory, Athens, Ga., EPA/600/R-97/080, p. 755.

Bishop D.J., Knezovitch, J.P., and Rice Jr., D.W. 1989. Sorption Studies of VOCs Related to Soil/Ground Water Contamination at LLNL. Environmental Protection Agency Environmental Restoration Series, UCID-21651:1-12. Lawrence Livermore National Laboratory, Livermore, California.

Bloom, Y., et al., 2002. Carbon isotope fractionation during microbial dechlorination of trichloroethene, cis-1,2-dichloroethene, and vinyl chloride: Implications for assessment of natural attenuation. Environ. Sci. and Technol., 34: 2768-2772.

Boopathy, R. and Peters, R. 2001. "Enhanced Biotransformation of Trichloroethylene Under Mixed Electron Acceptor Conditions." Current Microbiol., 42: 134-138.

Borden, R.C. 1994. Natural bioremediation of hydrocarbon-contaminated ground water. Pp. 177-199. In Matthews J.E. (ed) Handbook of Bioremediation. Lewis Publishers: Boca Raton. 
Boschker, H.T.S., et al. 1998. Direct linking of microbial populations to specific biogeochemical processes by 13C-labelling of biomarkers. Nature, 392: 801-805.

Bouwer, H. 1989. The Bouwer and Rice slug test - an update: Ground Water, 27(3): 304-309.

Bouwer, E. J. 1994. Bioremediation of chlorinated solvents using alternate electron acceptors. p. 149-175. In R. D. Norris et al. (eds.) Handbook of Bioremediation, Lewis Publishers, Boca Raton.

Boyajian, G.E. and Devedjian, D.L. 1997. "Phytoremediation: It Grows on You", Soil \& Groundwater Cleanup, February/March, p. 22-26.

Boyle, J.J. and J.R. Shann. 1995. Biodegradation of phenol, 2,4-DCP, 2,4-D and 2,4,5-T in fieldcollected rhizosphere and nonrhizosphere soils. J. Environ, Qual., 24: 782-785.

Bradley, P.M. 2000. "Microbial Degradation of Chloroethenes in Groundwater Systems." Hydrogeology, 8: 104-111.

Bradley, P.M., and Chapelle, F. H. 1996. Anaerobic mineralization of vinyl chloride in Fe (III)-reducing, aquifer sediments. Environ. Sci. and Technol., 30: 2084-2086.

Bradley, P.M., and Chapelle F.H. 1997. Kinetics of DCE and VC mineralization under methanogenic and Fe (III) -reducing conditions. Environ. Sci. and Technol., 31: 2692-2696.

Bradley, P.M., and Chapelle, F.H.. 1998a. Effect of contaminant concentration on aerobic microbial mineralization of DCE and VC in stream-bed sediments. Environ. Sci. and Technol., 32: 553-557.

Bradley, P. M., and Chapelle, F. H.. 1998b. Microbial mineralization of VC and DCE under different terminal electron accepting conditions. Anaerobe, 4: 81-87.

Bradley, P. M., Chapelle, F. H., and Wilson, J. T. 1998. Field and laboratory evidence for intrinsic biodegradation of vinyl chloride contamination in a Fe (III) -reducing aquifer. J. Contam. Hydrol., 31: 111-127.

Bradley, P. M., Landmeyer, J. E., and Dinicola, R. S. 1998c. Anaerobic oxidation of $\left[1,2-{ }^{14} \mathrm{C}\right]$ dichloroethene under Mn (IV)-reducing conditions. Appl. Environ. Microbiol., 64: 1560-1562.

Bradley, P.M. and Chapelle, F.H. 2000a. Aerobic microbial mineralization of dichloroethene as sole carbon substrate. Environ. Sci. and Technol., 34: 221-223.

Bradley, P.M. and Chapelle, F.H. 2000b. Acetogenic microbial degradation of vinyl chloride. Environ. Sci. and Technol., 34: 2761-2763.

Bradshaw, A.D. and Chadwick, M.J. 1980. The restoration of land: the geology and reclamation of derelict and degraded land. Univ. California Press, Berkeley.

Briggs, G.G., Bromilow, R.H., and Evans, A.A. 1982. Relationships between lipophilicity and root uptake and translocation of non-ionized chemicals by barley: Pesticide Science, 13: 495 - 504.

Brigmon, R.L., Bell, N.C., Freedman, D.L., and Berry, C.B. 1998. Natural Attenuation of Trichloroethylene in Rhizosphere Soils at the Savannah River Site. J. Cont. Soils, 7: 433-453.

Brigmon, R.L., Anderson, T.A., and Fliermans, C.B. 1999. "Methanotrophic Bacteria in the Rhizosphere of Trichloroethylene Degrading Plants". Int. J. Phytoremediation, 1: 241-253. 
Brigmon, R.L., Camper, D., and Stutzenberger, F.. 2002. "Bioremediation of Compounds Hazardous to Health and the Environment - An Overview." Biotransformations: Bioremediation Technology for Health and Environmental Protection, V. P. Singh ed. Elsevier Science Publishers, The Netherlands. p 1-28

Brock, T.D., Madigan, M.T., et al. 1994. Biology of Microorganisms. Englewood Cliffs, Prentice Hall.

Brusseau, M.L. and Rao, P.S.C. 1989. Sorption nonideality during organic contaminant transport in porous media. Crit. Rev. Environ. Control, 19(1): 33-99

Brusseau, M.L., Jessup, R.E., and Rao, P.S. 1991. "Nonequilibrium Sorption of Organic Chemicals: Elucidation of Rate-Limiting Processes.” Environ. Sci. and Technol., 25: 134-142.

Burgos, W.D., Novak, J.T., and Berry, D.F. 1996. Reversible Sorption and Irreverisible Binding of Naphthalene and $\alpha-N a p h t h o l$ to Soil: Elucidation of Processes. Environ. Sci. and Technol., 30: 1205-1211.

Burken, J.G., and Schnoor, J.L. 1996. Phytoremediation: Plant uptake of atrazine and role of root exudates. J. Env. Eng., 122: 958-963.

Burken, J.G., and Schnoor, J.L. 1998. Predictive Relationships for Uptake of Organic Contaminants by Hybrid Poplar Trees. Environ. Sci. and Technol., 32: 3379-3385.

Burton, T., Atekwana, Elliot A., Atekwana, Estella A. 2003 Mineral grain surface observations at a hydrocarbon-contaminated aquifer. SAGEEP 2003 Proceedings, 228-237.

Buscheck, T.E. and Alcantar, C.M. 1995. "Regression Techniques and Analytical Solutions to Demonstrate Intrinsic Bioremediation." In, Proceedings of the 1995 Battelle International Conference on In-Situ and On Site Bioreclamation.

Butler, B.J., and Barker, J.F. 1996. Chemical and microbiological transformation and degradation of chlorinated solvent compounds, In, Pankow, J.F., and Cherry, J.A., eds., Dense Chlorinated Solvents and Other DNAPLs in Ground water: History, Behavior, and Remediation: Waterloo Press, Waterloo, Ontario, p. 267-312.

Butler, E.C and Hayes, K.F. 2001. Factors influencing rates and products in the transformation of trichloroethylene by iron sulfide and iron metal. Environ. Sci. Technol., 35(19): 3884-3891.

Butler, E.C and Hayes, K.F. 2000. Kinetics of the transformation of halogenated aliphatic compounds by iron sulfide. Environ. Sci. and Technol., 34(3): 422-429.

Butler, E.C and Hayes, K.F. 1999. Kinetics of the transformation of trichloroethylene and tetrachloroethylene by iron sulfide. Environ. Sci. Technol., 33(12): 2021-2027.

Carmachael, L.M., Christman, R.F., and Pfaender, F.K. 1997. "Desorption and Mineralization Kinetics of Phenanthrene and Chrysene in Contaminated Soils." Environ. Sci. and Technol., 31: 126-132.

Carr, C.S. and Hughes, J.B. 1998. "Enrichment of High Rate PCE Dechlorination and Comparative Study of Lactate, Methanol, and Hydrogen as Electron Donors to Sustain Activity." Environ. Sci. and Technol., 32(12): 1817-1824. 
Carroll, K.M., Harkness, M.R., Bracco, A.A., and Balcarcel, R.R. 1994. Application of a permeant/polymer diffusional model to the desorption of polychlorinated biphenyls from Hudson river sediments. Environ. Sci. and Technol., 28(2): 253-258.

Cervini-Silva, J., Kostka, J.E., Larson, R.A., Stucki, J.W., and Wu, J. 2003. Dehydrochlorination of 1,1,1-trichloroethane and pentachloroethane by microbially reduced ferruginous smectite. Environ. Toxicol. Chem., 22(5): 1046-1050.

Chang, M.L., Wu, S.C., and Chen, C.Y. 1997. "Diffusion of Volatile Organic Compounds in Pressed Humic Acid Discs.” Environ. Sci. and Technol. 31: 2307-2312.

Chapelle, F.H., Bradley, P.M., Lovley D.R., and Vroblesky D.A. 1996a. Measuring rates of biodegradation in a contaminated aquifer using field and laboratory methods. Ground Water. 34: 691-698.

Chapelle, F.H., Haack, S.K., Adriaens, P., Henry, M.A., and Bradley, P.M.. 1996b. Comparison of Eh and $\mathrm{H}_{2}$ measurements for delineating redox processes in a contaminated aquifer. Environ. Sci. and Technol., 30: 3565-3569.

Chapelle, F.H., McMahon, P.B., Dubrovsky, N.M., Fujii, R.F., Oaksford, E.T., and Vroblesky, D.A. 1995. Deducing the distribution of terminal electron-accepting processes in hydrologically diverse groundwater systems. Water Resources Research, 31: 359-371.

Chapelle, F.H. 1993. Groundwater Microbiology and Geochemistry: John Wily \& Sons, Inc., New York, p. 424.

Chapelle, F.H., McMahon, P.B., Dubrovsky, N.M., Fujii, R.F., Oaksford, E.T., and Vroblesky, D.A. 1995. Deducing the distribution of terminal electron-accepting processes in hydrologically diverse groundwater systems: Water Resources Research, 31: 359-371.

Chapelle, F.H. 1996. Identifying redox conditions that favor the natural attenuation of chlorinated ethenes in contaminated ground-water systems. pp. 17-20. In Symposium on Natural Attenuation of Chlorinated Organics in Ground Water, EPA/540/R-96/509.

Chapelle, F.H., Vroblesky, D.A., Woodward, J.C., and Lovley, D.R. 1997. Practical considerations for measuring hydrogen concentrations in groundwater. Environ. Sci. and Technol., 31:2873-2877.

Chapelle, F. H. and Lovley, D.R. 1990. "Rates of Microbial-Metabolism in Deep Coastal-Plain Aquifers." Applied and Environmental Microbiology, 56(6): 1865-1874.

Chapelle, F.H. 1996. Identifying redox conditions that favor the natural attenuation of chlorinated ethenes in contaminated groundwater systems, In Proceedings of the Symposium on Natural Attenuation of Chlorinated Organics in Groundwater, Dallas, TX: EPA/540/R-96/509.

Chapelle, F.H., Vroblesky, D.A., Woodward, J.C., and Lovley, D.R. 1997. Practical considerations for measuring hydrogen concentrations in groundwater: Environ. Sci. and Technol., 31: 2873-2877.

Chapelle, F.H. and Bradley, P.M. 1998. Selecting remediation goals by assessing the natural attenuation capacity of groundwater systems. Bioremediation Journal, 2: 227-238.

Chapra, S.C. 1996. Surface Water-Quality Monitoring, p. 844 New York, NY: McGraw Hill. 
Charbeneau, R.J. and Daniel, D.E. 1993. Contaminant Transport in Unsaturated Flow, In: Handbook of Hydrology, p 151 (Maidment, D.R. Ed.). New York, NY: McGraw-Hill.

Chen, F., B. Binder, and Hodson, R.E., 2000. Flow cytometric detection of specific gene expression in prokaryotic cells using in situ RT-PCR. FEMS Microbiology Letters, 184: 291-295.

Chen, W., A.T. Kan, C.J. Newell, E. Moore, and M.B. Tomson. 2002. More realistic soil cleanup standards with dual-equilibrium desorption. Ground Water, 40(2):153-164.

Chiou, C.T., Porter, P.E., and. Schmedding, D.W. 1983. "Partition Equilibria of Nonionic Organic Compounds Between Soil Organic Matter and Water." Environ. Sci. Technol., 17: 227-231.

Chiou, C.T., and Shoup, T.D. 1985. "Soil Sorption of Organic Vapors and Effects of Humidity on Sorptive Mechanism and Capacity." Environ. Sci. and Technol., 19: 1196.

Clement, T.P. 1997. RT3D - A Modular Computer Code for Simulating Reactive Multi-Species Transport in 3-Dimensional Groundwater Aquifers. Pacific Northwest National Laboratory, Richland, WA. PNNL-11720.

Clement, T.P., Sun, Y., Hooker, B.S., and Petersen, J.N. 1998. Modeling Multi-Species Reactive Transport in Groundwater Aquifers. Groundwater Monitoring \& Remediation Journal, 18(2): 7992.

Cline, P.V., and Delfino, J.J., 1989, Transformation kinetics of 1,1,1-trichloroethane to the stable product 1,1-dichloroethene, In: Biohazards of Drinking Water Treatment. Lewis Publishers, Inc. Chelsea, MI. p. 47-56.

Cohen, R.M. and Mercer, J.M. 1993. DNAPL Site Evaluation. CRC Press, Inc., Boca Raton, Florida.

Coleman, N.V., et al. 2002. Biodegradation of cis-dichloroethene as the sole carbon source by a BProteobacterium. Applied and Environmental Microbiology, 68: 2726-2730.

Conant Jr., B. 2001. A PCE Plume Discharging to a River: Investigations of Flux, Geochemistry, and Biodegradation in the Streambed. PhD Dissertation, Earth Sciences, University of Waterloo, Waterloo Ontario Canada.

Corley, T.L., Farrell, J., Hong, B., and Conklin, M.H. 1996. "VOC Accumulation and Pore Filling in Unsaturated Porous Media." Environ. Sci. and Technol., 30: 2884-2891.

Cornelissen, G., Van Noort, P.C.M., Parsons, J.R., and Govers, H.A.J.. 1997. Temperature Dependence of Slow Adsorption and Desorption Kinetics of Organic Compounds in Sediments." Environ. Sci. and Technol., 31: 454-460.

Cornelissen, G., Van Noort, P.C.N., and Govers, H.A.J. 1998. "Mechanism of Slow Desorption of Organic Compounds From Sediments: A Study Using Model Sorbents." Environ. Sci. and Technol., 32: 3124-3131.

Cornett, R., James, M.O., Henderson, G.N. and Stacpoole, P.W. 1999. "Inhibition of glutathione-Stransferase-zeta and tyrosine metabolism by dichloroacetate: a potential unifying mechanism for its altered biotransformation and toxicity." Biochemical and Biophysical Research Communications, 262: 752-756 
Corry, C.E. 1985. Spontaneous polarization associated with porphyry mineralization. Geophysics, 50: 1020-1034.

Cowan, D.A., 2000. Microbial genomes - the untapped resource. Trends in Biotechnology, 18: 14-16.

Cozarelli, I.M., Berkins, B.A., Baedecker, M.J., Aiken, G.R., Eganhouse, R.P., and Tuccillo, M.E. 2001. Progression of natural attenuation processes at a crude oil spill site: I. Geochemical evolution of the plume. Journal of Contaminant Hydrogeology, 53: 369-385.

Culver, T.B., Hallisey, S.P., Sahoo, D., Deitsch, J.J., and Smith, J.A. 1997. Modeling the desorption of organic contaminants from long-term contaminated soil using distributed mass transfer rates. Environ. Sci. and Technol., 31(6): 1581-1588.

Cunningham, S.D., Anderson, T.A., Schwab, A.P., and Hsu F.C. 1996. Phytoremediation of soils contaminated with organic pollutants. Advances in Agronomy, 56: 55-107.

Cupples, A.M., Spormann, A.M., and McCarty, P.L. 2003. Growth of a Dehalococcoides - like microorganism on vinyl chloride and cis - dichloroethene as electron acceptors as determined by competitive PCR. Applied and Environmental Microbiology, 69: 953-959.

Dahm, C.N., Grimm, N.B., Marmonier, P., Valett, M.H., and Vervier, P. 1998. Nutrient dynamics at the interface between surface waters and groundwaters. Freshwater Biology, 40: 427-451.

Davis, J.W., and Carpenter, C.L. 1990. Aerobic biodegradation of vinyl chloride in groundwater samples. Appl. Environ. Microbiol., 56: 3870-3880.

Davis, L.D., Lupher, D., Hu, J., and Erickson, L.E. 1999. Transport of trichloroethylene through living plant tissues; in, Erickson, L.E. and Rankin, M.M., eds., Proceedings of the 1999 Conference on Hazardous Waste Research: Kansas State University, Manhattan, Kansas (http://www.engg.ksu.edu/HSRC), p. 219 - 223.

De Bruin, W. P., Kotterman, M. J. J., Posthumus, M. A., Schraa, G., and Zehnder, A. J. B. 1992. Complete biological reductive transformation of tetrachloroethene to ethane. Appl. Environ. Microbiol., 58: 1996-2000.

Deitsch, J.J., J.A. Smith, Arnold, M.B., and Bolus, J. 1998. "Sorption and Desorption of Carbon Tetrachloride and 1,2-Dichlorobenzene to Three Organibentonites and a Natural Peat Soil." Environ. Sci. and Technol., 32: 3169-3177.

Deitsch, J.J., Smith, J.A., Culver, T.B., Brown, R.A., and Riddle, S.A. 2000. "Distributed-Rate Model Analysis of 1,2-Dichlorobenzene Batch Sorption and Desorption Rates for Five Natural Sorbents." Environ. Sci. and Technol., 34: 1469-1476.

Diaz-Torres, M.L., et al. 2003. Novel tetracycline resistance determinant from the oral metagenome. Antimicrobial Agents and Chemotherapy, 47: 1430-1432.

Dickmann, D.I., and Stuart, K.W. 1983. The Culture of Poplars in Eastern North America. Hickory Hollow Associates, Dansville, MI. 
Dickmann, D.I., Nguyen, P.V., and Pregitzer, K.S. 1996. Effects of irrigation and coppicing on aboveground growth, physiology, and fine-root dynamics of two field-grown hybrid poplar. For. Ecol. and Manage, 80: 163-174.

Dietz, A.C. and Schnoor, J.L., 2001a, Advances in phytoremediation: Environmental Health Perspective, Supplement 1, 109: $163-168$.

Dietz, A.C. and Schnoor, J.L., 2001b, Phytotoxicity of chlorinated aliphatics to hybrid popular (Populus deltoids x Nigra DN34): Environmental Toxicology and Chemistry, 20: 389 - 393.

Dillings, W.L., Tfertiller, N.B., and Kallos, G.J., 1975, Evaporation rates and reactivities of methylene chloride, chloroform, 1,1,1-trichloroethane, trichloroethylene, tetrachloroethylene, and other chlorinated compounds in dilute aqueous solutions: Environ. Sci. and Technol., 9: 833 - 838.

DiStefano, T. D., Gossett, J. M., and Zinder, S. H. 1991. Reductive dechlorination of high concentrations of tetrachloroethene to ethene by an anaerobic enrichment culture in the absence of methanogenesis. Appl. Environ. Microbiol., 57: 2287-2292.

Dojka, M.A., et al. 1998. Microbial diversity in a hydrocarbon- and chlorinated-solvent-contaminated aquifer undergoing intrinsic bioremediation. Applied and Environmental Microbiology, 1998. 64: 3869-3877.

Doty, S.L., Shang, T.Q., Wilson, A.M. Tangen, J., Westergreen, A.D., Newman, L.A., Strand, S.E. and Gordon, M.P. 2000. Enhanced metabolism of halogenated hydrocarbons in transgenic plants containing mammalian cytochrome P450 2E1. Proc. Natl. Acad. Sci., 97: 6287-6291.

Doty, S.L., Shang, T.Q., Wilson, A.M., Moore, A.L., Newman, L.A., Strand, S.E., and Gordon, M.P. 2003. Metabolism of the soil and groundwater contaminants, ethylene dibromide and trichloroethylene, by the tropical leguminous tree, Leuceana leucephala. Water Res., 37: 441-449.

Doty, S.L., Shang, T.Q., Wilson, A.M., Tangen, J., Westergreen, A.D., Newman,L.A., Strand, S.E., and Gordon, M.P. 2000. Proc. Nat. Acad. Sci., 97: 6287-6291.

Drzyzga, O., et al. 2001 Coexistence of a sulphate-reducing Desulfovibrio species and the dehalorespiring Desulfitobacterium frappieri TCE1 in defined chemostat culture grown with various combinations of sulphate and tetrachloroethene. Environmental Microbiology, 3: 92-99.

Drzyzga, O. and Gottschal, J.C. 2002. Tetrachloroethene dehalorespiration and growth of Desulfitobacterium frappieri TCE1 in strict dependence on the activity of Desulfovibrio fructosivorans. Applied and Environmental Microbiology, 68: 642-649.

Duhamel, M., et al., 2002. Comparions of anaerobic dechlorinating enrichment cultures maintained on tetrachloroethene, trichloroethene, cis-dichloroethene and vinyl chloride. Water Research, 36: 4193-4202.

Duineveld, B.M., et al., 2001. Analysis of bacterial communities in the rhizosphere of chrysanthemum via denaturing gradient gel electrophoresis of PCR-amplified 16S rRNA as well as DNA fragments for 16S rRNA. Applied and Environmental Microbiology, 67: 172-178.

Ellis, D. E., Lutz, E. J., et al. 2000. "Bioaugmentation for Accelerated In Situ Anaerobic Bioremediation." Environ. Sci. and Technol., 34(11): 2254-2260. 
Falta, R.W., Javandal, I., Pruess, J., and Witherspoon, P.A. 1989. Density-driven flow of gas in the evaporation of volatile organic compounds: Water Resources Research, 25: 2159 - 2169.

Fan, S., and Scow, K.M. 1993. Biodegradation of trichloroethylene and toluene by indigenous microbial populations in soil. App. Environ. Microbiol., 59: 1911-1918.

Fan, T.W-M., Higashi, R.M., Lane, A.N. 2000. "Chemical characterization of a chelator-treated soil humate by solution-state multinuclear two-dimensional NMR with FTIR and pyrolysis-GCMS", Environ. Sci. and Technol., 34: 1636-1646

Fantroussi, S.E., Naveau, H., and Agathos, S.N. 1998. Anaerobic Dechlorinating Bacteria. Biotechnology Progress, 14: 167-188.

Farrell, J. and Reinhard, M. 1994a Desorption of Halogenated Organics from Model Solids, Sediments and Soil Under Unsaturated Conditions. 1. Isotherms. Environ. Sci. and Technol., 28: 53-62.

Farrell, J. and Reinhard, M.. 1994b Desorption of Halogenated Organics from Model Solids, Sediments, and Soil Under Unsaturated Conditions. 2. Kinetics. Environ. Sci. and Technol., 28: 63-72.

Farrell, J., Grassian, D., and Jones, M.. 1999. Investigation of Mechanisms Contributing to Slow Desorption of Hydrophobic Organic Compounds from Mineral Solids. Environ. Sci and Technol., 33: $1237-1243$.

Fennell, D.E., Gossett, J.M., et al. 1997. "Comparison of Butyric Acid, Ethanol, Lactic Acid, and Propionic Acid as Hydrogen Donors for the Reductive Dechlorination of Tetrachloroethene." Environ. Sci. and Technol., 31(3): 918-926.

Fennell, D.E., Stover, M.A., Zinder, S.H., and Gossett, J.M. 1995. Comparison of alternative electron donors to sustain PCE anaerobic reductive dechlorination. p. 9-16. In R. E. Hinchee, A. Leeson, and L. Semprini (eds.) Bioremediation of chlorinated solvent, Battelle Press, Columbus.

Fennell, D.E., et al. 2001. Assessment of indigenous reductive dechlorinating potential at a TCEcontaminated site using microcosms, polymerase chain reaction analysis, and site data. Environ. Sci. and Technol. 35: 1830-1839.

Finneran, K.T., Forbush, H.R., et al. 2002. "Desulfitobacterium metallireducens sp. nov., an Anaerobic Bacterium that Couples Growth to the Reduction of Metals and Humic Acids as well as Chlorinated Compounds." Int. J. Syst. Evol. Microbiol., 52: 1929-1935.

Fletcher, J. S. and Hegde, R. S. 1995. Release of phenols by perennial plant roots and their potential importance in bioremediation. Chemosphere, 31:3009-3016.

Fliermans, C.B., Phelps, T.J., Ringelberg, D., Mikell, A.T., and White, D.C. 1988. Mineralization of trichloroethylene by heterotrophic enrichment cultures. Appl. Environ. Microbiol., 54: 1709-1714.

Flynn, S.J., Loffler, F.E., and Tiedje, J.M. 2000. Microbial community changes associated with a shift from reductive dechlorination of PCE to reductive dechlorination of cis-DCE and VC. Environ. Sci. and Technol., 34: 1056-1061. 
Freedman, D.L., and Gossett, J.M. 1989. Biological reductive dechlorination of tetrachloroethylene and trichloroethylene to ethylene under methanogenic conditions. Appl. Environ. Microbiol., 55: 21442151.

Freeze, R.A. and Cherry, J.A.1979. Groundwater. Prentice Hall, Englewood Cliffs, New Jersey. 604 pgs.

Fuller, M.E., Mu, D.Y., and Scow, K.M. 1995. Biodegrdation of trichloroethylene and toluene by indigenous microbial populations in vadose sediments. Microb. Ecology, 29: 311-325.

Gander, J.W., Parkin, G.F., and Scherer, M.M. 2002. Kinetics of 1,1,1-Trichlorethane transformation by iron sulfide and methanogenic consortium: Environ. Sci. and Technol., 36: 4540 - 4546.

Garbarini, D.R., and Lion, L.W. 1985. Evaluation of sorptive partitioning of nonionic pollutants in closed systems by headspace analysis: Environ. Sci. and Technol., 19: $1122-1128$.

Garbarini, D.R., and Lion, L.W. 1986. The influence of the nature of soil organics on the sorption of toluene and trichloroethene: Environ. Sci. and Technol., 20: 1263 - 1269.

Gelhar, L.W., Welty, C., and Rehfeldt, K.R. 1992. A Critical Review of Data on Field-Scale Dispersion in Aquifers. Water Resources Research, 28: 955-1974.

Genereux, D.P., and Hemond, H.F. 1990. Naturally occurring radon-222 as a tracer for streamflow generation: steady state methodology and field example. Water Resources Research, 26(12): 3065-3075.

Gerlach, R., Cunningham A.B., and Caccavo Jr., F. 2000. Dissimilatory iron-reducing bacteria can influence the reduction of carbon tetrachloride by iron metal. Environ. Sci. and Technol., 34(12): 2461-2464.

Gerritse, J. Drzyzga, O., et al. 1999. "Influence of Different Electron Donors and Acceptors on Dehalorespiration of Tetrachloroethene by Desulfitobacterium frappieri TCE1." Appl. Environ. Microbiol., 65(12): 5212-5221.

Gerritse, J.,V. Renard, et al. 1996. "Desulfitobacterium sp. strain PCE1, an Anaerobic Bacterium that can Grow by Reductive Dechlorination of Tetrachloroethene or ortho-Chlorinated Phenols." Arch. Microbiol., 165: 132-140.

Gilbert, R.O. 1987. "Statistical Methods for Environmental Pollution Monitoring," Van Nostrand Reinhold, New York.

Gill, I. and Ballesteros, A. 2000. Bioencapsulation within synthetic polymers (Part 1): sol-gel encapsulated biologicals. Trends in Biotechnology, 18: 282-296.

Gillham, R.W., and O’Hannesin, S.F. 1994. Enhanced degradation of halogenated aliphatics by zerovalent iron: Ground Water, 32(6): 958-967.

Gordon, M.P., Choe, N., Duffy, J., Ekuan, G., Heilman, P., Muizniecks, P., Ruszaj, M., Shurtleff, B.B., Strand, S., Wilmoth, J., and Newman, L.A. 1998. Phytoremediationof trichloroethylene with hybrid poplars. Env. Health Pers., 106: 1001-1004.

Gossett, J.M. 1998. "Modeling the Production of and Competition for Hydrogen in a Dechlorinating Culture." Environ. Sci. and Technol., 32(16): 2450-2460. 
Gossett, J. M., and Zinder S. H. 1996. Microbiological aspects relevant to natural attenuation of chlorinated ethenes. pp. 10-13. In Symposium on Natural Attenuation of Chlorinated Organics in Ground Water, EPA/540/R-96/509.

Gossett, J.M. 2002. Fishing for microbes. Science, 298: 974-975.

Grant, W.B., Optical Remote Measurement of Toxic Gases, Journal of Air \& Waste Management Association, 42(1): 18.

Groundwater Services, Inc. 1998. Remediation by Natural Attenuation (RNA) ToolKit Users Manual, Groundwater Services, Inc., Houston, Texas.

Haag, W.R., and Mill, T. 1988. Transformation kinetics of 1,1,1-trichloroethane to the stable product 1,1dichloroethene: Environ. Sci. and Technol., 22: 658-663.

Hadley, P.W. and Armstrong, R. 1991. "Where's the benzene?"-Examining California ground-water quality survey. Ground Water, 20: 35-40.

Hansen, E.A. 1992. Mid-rotation yields of biomass plantations in the north central United States. U.S.D.A. Forest Service Res. Pap., NC-309 8 p.

Hansen, E.A. 1993. Soil carbon sequestration beneath hybrid poplar plantations in the north central United States. Biomass and Bioenergy, 5: 431-436.

Harkness, M.R., et al. 1999. Use of bioaugmentation to stimulate complete reductive dechlorination of trichloroethene in dover soil columns. Environ. Sci. and Technol., 33: 1100-1109.

Hartmans, S. 1995. Microbial degradation of vinyl chloride. In V. P. Singh (ed.) Biotransformations: Microbial Degradation of Health Risk Compounds, Elsevier Science. p. 239-248.

Hartmans, S., J. deBont, A. M., Tramper, J., and Luyben, K. C. A. M. 1985. Bacterial degradation of vinyl chloride. Biotechnol. Lett., 7: 383-388.

Hartmans, S., and deBont, J. A. M. 1992. Aerobic vinyl chloride metabolism in Mycobacterium aurum L1. Appl. Environ. Microbiol., 58: 1220-1226.

Haston, Z. C. and McCarty, P. 1999. "Chlorinated Ethene Half-Velocity Coefficients (Ks) for Reductive Dehalogenation." Environ. Sci. and Technol. 33(2): 223-226.

Haston, Z. C., Sharma, P. K., Black, J. N., and McCarty, P. L. 1994. Enhanced reductive dechlorination of chlorinated ethenes. In U.S. EPA. Bioremediation of hazardous wastes, USEPA, San Francisco.

Hatfield, K., Annable, M.D., Kuhn, S., Rao, P.S.C., and Cambell, T. 2002. A new method for quantifying contaminant flux at hazardous waste sites, Groundwater Quality 2001, IAHS Red Book, Shefield United Kingdom.

He, J., et al. 2003. Complete detoxification of vinyl chloride by an anaerobic enrichment culture and identification of the reductively dechlorinating population as a Dehalococcoides species. Applied and Environmental Microbiology, 69: 996-1003.

Head, I.M., Saunders, J.R., and Pickup, R.W. 1998. Microbial Evolution, Diversity, and Ecology: A Decade of Ribosomal RNA Analysis of Uncultivated Microorganisms. Microbial Ecology, 35: 121. 
Hegde, R. S. and Fletcher, J. S. 1996. Influence of plant growth stage and season on the release of root phenolics by mulberry as related to development of phytoremediation technology. Chemosphere. 32: $2471-2479$.

Heilman, P.E., and Stettler, R.F. 1985. Genetic variation and productivity of Populus trichocarpa and its hybrids. II. Biomass production in a 4-year plantation. Can. J. For. Res., 15: 382-388.

Heilman, P.E., and Stettler, R.F. 1990. Genetic variation and productivity of Populus trichocarpa and its hybrids. IV. Performance in short-rotation coppice. Can. J. For. Res., 20: 1257-1264.

Hem, J.D. 1985. Study and Interpretation of the Chemical Characteristics of Natural Water: United States Geological Survey Water Supply Paper 2254, p. 264.

Hendrickson, E. R., Payne, J., et al. 2002. "Molecular Analysis of Dehalococcoides 16S Ribosomal DNA from Chloroethene-Contaminated Sites throughout North America and Europe." Appl Environ Microbiol., 68(2): 485-495.

Hendrickson, E.R., et al. 2001. Using a molecular approach to monitor a bioaugmentation pilot, in Bioaugmentation, Biobarriers, and Biogeochemistry, Batelle Press: San Diego. p. 43-51.

Hiltner, L. 1904. Uber neue erfahrungen und probleme auf dem gebiet der bodenbakteriolgie und unter besonderes berucksichtigung der grundugungen und brache. Arb. DtschLandwirt. Ges.. Berlin 98:59-778.

Hohnstock-Ashe, A.M., et al. 2001. Further biogeochemical characterization of a trichloroethenecontaminated fractured dolomite aquifer: electron source and microbial communities involved in reductive dechlorination. Environ. Sci. and Technol., 35: 4449-4456.

Holliger, C., Wohlfarth, G., and Diekert, G. 1999. Reductive dechlorination in the energy metabolism of anaerobic bacteria. FEMS Microbiology Reviews, 22: 383-398.

Holliger, C., Schraa, G., Stams, A. J. M., and Zehnder, A. J. B. 1993. A highly purified enrichment culture couples the reductive dechlorination of tetrachloroethene to growth. Appl. Environ. Microbiol., 59: 2991-2997.

Hong, M.S., Farmayan, W.F., Dortch, I.J., Chian, C.Y., McMillan, S.K., and Schnoor, J.L. 2001. Phytoremediation of MTBE from a groundwater plume: Environ. Sci. and Technol., 35: 12311239.

Hopkins, G.D., and McCarty P.L. 1995. Field evaluation of in situ aerobic cometabolism of trichloroethylene and three dichloroethylene isomers using phenol and toluene as the primary substrates. Environ. Sci. and Technol., 29: 1628-1637.

Hsu, T.S. and Bartha H. 1979. Accelerated mineralization of two organophosphorous insecticides in the rhizosphere. Appl. Environ. Microbiol., 37: 36-41.

Huang, W., and Weber Jr., W.J., 1997. "A Distributed Reactivity Model for Sorption by Soils and Sediments. 10. Relationships Between Desorption, Hysteresis, and the Chemical Characteristics of Organic Domains.” Environ. Sci. and Technol., 31: 2562-2569. 
Hugenholtz, P., Goebel, B.M., and Pace, N.R. 1998. Impact of culture-independent studies on the emerging phylogenetic view of bacterial diversity. Journal of Bacteriology, 180: 4765-4774.

Hurt, R.A., et al., 2001. Simulataneous recovery of RNA and DNA from soils and sediments. Applied and Environmental Microbiology, 67: 4495-4503.

Interstate Technology and Regulatory Cooperation Work Group 1999. Phytoremediation - Decision Tree: Interstate Technology \& Regulatory Council accesses at http://www.itrcweb.org on June 30, 2003.

ITRC Re-release, 1998. ISB Protocol Binder \& Resource Document for Hydrocarbons, June 1996.

ITRC, Technical and Regulatory Requirements for Enhanced In Situ Bioremediation of Chlorinated Solvents in Groundwater, December 1998.

ITRC, Five-Course Evaluation Summary for the ITRC/RTDF Training Course: Natural Attenuation of Chlorinated Solvents in Groundwater, September 1999.

ITRC, Natural Attenuation of Chlorinated Solvents in Groundwater: Principles and Practices, September 1999.

ITRC Final Report, Recommendations for the use of polyethylene diffusion bag samplers for the longterm monitoring of volatile organic compounds in groundwater, Nov. 6, 2002.

Jackson, D. G., T. Payne, B. B. Looney and J. Rossabi, 1996, Estimation of the Extent and Thickness of DNAPL in the A/M Area. Report No. WSRC-RP-96-0574, U. S. Department of Energy Office of Scientific and Technical Information, Oak Ridge TN.

Jafvert, C.T., and Wolfe, N.L. 1987. Degradation of selected halogenated ethanes in anoxic sedimentwater systems: Environ. Toxicol. Chem., 6: 827-837.

James, M.O., Cornett, R., Yan, Z., Henderson, G.N. and Stacpoole, P.W. 1997. "Glutathione-dependent conversion to glyoxylate, a major pathway of dichloroacetate biotransformation in hepatic cytosol from humans and rats, is reduced in rats by pretreatment with DCA." Drug Metabolism and Disposition, 25: 1223-1227.

Jeffers, P.M., Ward, L.M., Woytowitch, L.M., and Wolfe, N.L. 1989. Homogeneous hydrolysis rate constants for selected chlorinated methanes, ethanes, ethenes, and propanes: Environ. Sci. and Technol., 23: 965-969.

Jeffers P.M., Coty, P., Luczak, S., and Wolf, N.L. 1994. Halocarbon hydrolysis rates-a search for ionic strength and heterogeneous effects. J. Environ. Sci. Health, A29(4):821-831.

Jeffers P.M., Brenner, C., and Wolf, N.L. 1996. Hydrolysis of Carbon Tetrachloride. Environ. Toxicol. Chem., 15(7): 1064-1065.

Jeng C.Y., Chen, D.H., and Yaws, C.L. 1992. Data Compilation for Soil Sorption Coefficient. Pollution Engineering, p. 54-60.

Johnson, R.L., Palmer, C.D., and Fish, W. 1989. Subsurface chemical processes, In Fate and Transport of Contaminants in the Subsurface, EPA/625/4-89/019: Environmental Protection Agency, Cincinnati, $\mathrm{OH}$ and Ada, OK, p. 41-56. 
Jones Jr., J. B. and R.M. 1996. Surface-subsurface interactions in stream ecosystems. Trends in Ecology and Evolution 16: 239-242.

Joos, F., Plattner, G., Stocker, T.F., Kortzinger, A., and Wallace, D.W.R. 2003. Trends in Marine Dissolved Oxygen: Implications for Ocean Circulation Changes and the Carbon Budget, EOS, 84(21): 27.

Jury, W.A., Spencer, W.F., and Farmer, W.J. 1983. Behavior assessment model for trace organics in soil: I. Model description: Journal of Environmental Quality, 12: 558 - 564.

Kampbell, D. H., Hansen, J.E., Wiedemeier, T.H., and Henry, B.M. 2001. Natural Attenuation of Chlorinated Solvents at Multiple Air Force Base Demonstration Sites, 2001 Conference on Environmental Research, U. S. Department of Defense.

Kan, A.T., Fu, G., Hunter, M.A., and Tomson, M.B. 1997. Irreversible Adsorption of Naphthalene and Tetrachlorobiphenyl to Lulu and Surrogate Sediments. Environ. Sci. and Technol., 31: 2176-2185.

Kan, A.T., Fu G., Hunter, M., Chen, W., Ward, C.H., and Tomson, M.B. 1998. Irreversible Sorption of Neutral Hydrocarbons to Sediments: Experimental Observations and Model Predictions. Environ. Sci. and Technol., 32: 892-902.

Karapanagioti, H.K., Kleineidam, S., Sabatini, D.A., Grathwohl, P., and Ligouis, B. 2000. "Impacts of Heterogeneous Organic Matter on Phenanthrene Sorption: Equilibrium and Kinetic Studies with Aquifer Material." Environ. Sci. and Technol., 34: 406-414.

Karickhoff, S.W., Brown, D.S., and Scott, T.A. 1979. Sorption of hydrophobic pollutants on natural sediments. Water Res., 13: 241-248.

Katayama, A. and Matsumura F. 1993. Environ. Toxicol. Chem., 12: 1059-1065.

Kaufman, W.J. and Orlob, G.T. 1956. Measuring groundwater movement with radioactive and chemical tracers: American Water Works Association Journal, 48: 559-572.

Kile D.E., Chiou, C.T., Zhou, H., Li, H., and Xu, O. 1995. Partition of nonpolar organic pollutants from water to soil and sediment organic matters. Environ. Sci. and Technol., 29: 1401-1406.

Kim, H., Annable, M.D., and Rao, P.S.C. 2001. Gaseous transport of volatile organic chemicals in unsaturated porous media: effect of water-partitioning and air-water interfacial adsorption: Environ. Sci. and Technol., 35: 4457-4462.

Kitanidis, P.K., Semprini, L., Kampbell, D.H., and Wilson, J.T. 1993. Natural anaerobic bioremediation of TCE at the St Joseph, Michigan, Superfund site. p. 47-50. In U.S. EPA. Symposium on bioremediation of hazardous wastes: Research, development, and field evaluations. EPA/600/R93/054. Washington, DC.

Kleineidam, S., Rugner, H., Ligouis, B., and Grathwohl, P. 1999. "Organic Facies and Equilibrium Sorption of Phenanthrene." Environ. Sci. and Technol., 33: 1637-1644.

Klute, A. and Dirksen, C. 1986. Hydraulic conductivity and diffusivity: Laboratory methods. In: Klute, A ed. Methods of Soil Analysis, Part 1 - Physical and Mineralogical Methods, second edition. 
American Society of Agronomy, Inc., Soil Science Society of America, Inc., Madison, WI. 687734.

Knaebel, D.B. and Vestal, J.R. 1992. Effects of intact rhizosphere microbial communities on the mineralization of surfactants in surface soils. Can J. Microbiol., 38: 643-653.

Knox, R.C., Sabatini, D.A., and Canter, L.W. 1993. Subsurface Transport and Fate Processes. Lewis Publishers, Boca Raton, Florida, 430 p.

Koizumi, Y., et al. 2002. Parallel characterization of anaerobic toluene- and ethylbenzene degrading microbial consortia by PCR-denaturing gradient gel electrophoresis, RNA-DNA membrane hybridization, and DNA microarray technology. Applied and Environmental Microbiology, 68: 3215-3225.

Kondoh, A. and Nishiyama, J. 2000. Changes in hydrological cycle due to urbanization in the suburb of Tokyo metropolitan area, Japan. Advanced Space Research, Elsevier Science, Ltd. 26(7): 11731176.

Kriegman-King, M.R. and Reinhard, M. 1992. Transformation of carbon tetrachloride in the presence of sulfide, biotite, and vermiculite. Environ. Sci. and Technol., 26(11): 2198-2206.

Krumholz, L. R., Sharp, R., and Fishbain, S. S. 1996. A freshwater anaerobe coupling acetate oxidation to tetrachloroethylene dehalogenation. Appl. Environ. Microbiol. 62: 4108-4113.

Kruseman, G.P. and de Ridder, N.A. 1990. Analysis and Evaluation of Pumping Test Data. International Institute for Land Reclamation and Improvement Publication 47, Wageningen, The Netherlands.

Lange, C.C., et al., 1998. Engineering a recombinant Deinococcus radiodurans for organopollutant degradation in radioactive mixed waste environments. Nature Biotechnology, 16: 929-933.

Larkin, R.G. and Sharp Jr., J.M. 1992. On the relationship between river basin geomorphology, aquifer hydraulics, and ground-water flow direction in alluvial aquifers. Geological Society of America Bulletin, 104: 1608-1620.

Larson, R.A., and Weber, E.J. 1994. Reaction Mechanisms in Environmental Organic Chemistry. Lewis Publishers, Boca Raton, Florida, 433p.

Leaphart, A.B. and Lovell, C.R. 2003. Formyltetrahydrofolate synthetase sequences from salt marsh plant roots reveal a diversity of acetogenic bacteria and other bacterial functional groups. Applied and Environmental Microbiology, 69: 693-696.

Lee, C.R., Hoppel, R.E., Hunt, P.G. and Carlson, C.A. 1976. Feasibility of functional us of vegetation to filter, dewater and remove contaminants from dredged material. Techn. Rep. D-76-4 (June). US Army Engineer Waterways Expt Stn., Vicksburg, MS.

Lee, G. F., Jones-Lee A. 1997. Hazardous Chemical Site Remediation Through Capping: Problems with Long Term Protection, Remediation, 7(4): 51-57.

Lee J., Reeves, K.D., Brooks, R.R., and Jaffre, T. 1977. Isolation and identification of a citrato-complex of nickel from nickel-accumulating plants. Phytochem., 16: 1503-1505. 
Lee, N., et al. 1999. Combination of fluorescent in situ hybridization and microautoradiography - a new tool for structure-function analyses in microbial ecology. Applied and Environmental Microbiology, 65: 1289-1297.

Lee, W. and Batchelor, B. 2002a. Abiotic reductive dechlorination of chlorinated ethylenes by ironbearing soil minerals; 1 - pyrite and magnetite: Environ. Sci. Technol., 36: 5147-5154.

Lee, W. and Batchelor, B. 2002b. Abiotic reductive dechlorination of chlorinated ethylenes by ironbearing soil minerals; 2 - green rust: Environ. Sci. Technol., 36: 5348-5354.

Lee, W. and Batchelor, B. 2003. Reductive capacity of natural reductants, Environ. Sci. Technol., 37: 535-541.

Lenczewski, M., Jardine, P., McKay, 1., and Layton A. 2003. Natural attenuation of trichloroethylene in fractured shale bedrock. Journal of Contaminant Hydrology, 64: 151-168.

Lin, T.F., Van Loy, M.D., and Nazaroff, W.W. 1996. "Gas-Phase Transport and Sorption of Benzene in Soil.” Environ. Sci. and Technol., 30: 2178-2186.

Lincoff, A.H., and Gossett, J.M. 1984. The determination of Henry's Law constant for volatile organics by equilibrium partitioning in closed systems; in, W. Brutsaert and G.H. Jirka, eds., Gas transfer at water surfaces: Reidel Publishing Company, Germany, p. $17-25$.

Lipski, A., Friedrich, U., and Altendorf, K. 2001. Application of rRNA-targeted oligonucleotide probes in biotechnology. Applied and Environmental Microbiology, 56: 40-57.

Löffler, F.E., Sun, Q., Li, J., and Tiedje, J.M. 2000. “16S rRNA gene-based detection of tetrachloroethene (PCE)-dechlorinating Desulfuromonas and Dehalococcoides species." Appl. Environ. Microbiol., 66: 1369-1374.

Loffler, F.E., Ritalahti, K.M., and Tiedje, J.M. 1997. Dechlorination of chloroethenes is inhibited by 2bromoethanesulfonate in the absence of methanogens. Applied and Environmental Microbiology, 63: 4982-4985.

Loffler, F.E., Sanford, R.A., and Tiedje, J.M. 1996. Initial characterization of a reductive dehalogenase from Desulfitobacterium chlororespirans Co23. Applied and Environmental Microbiology, 62: 3809-3813.

Looney, B.B. (ed.), 2002. Technical Targets - A Tool to Support Strategic Planning in the Subsurface Contaminants Focus Area. WSRC-RP-2002-00077, U.S. Department of Energy Office of Environmental Management, available from the Office of Scientific and Technical Information, Oak Ridge TN.

Lorenz, P., et al. 2002. Screening for novel enzymes for biocatalytic processes: accessing the metagenome as a resource of novel functional sequence space. Current Opinion in Biotechnology, 13: $572-577$.

Lovley, D.R., Chapelle, F.H., and Woodward, J.C. 1994. Use of dissolved $\mathrm{H}_{2}$ concentrations to determine distribution of microbially catalyzed redox reactions in anoxic groundwater. Environ. Sci. Technol., 28(7): 1205-1210. 
Lovley, D.R. 1991. "Dissimilatory Fe (III) and Mn (IV) Reduction." Microbiol., Rev. 55(2): 259-287.

Lovley, D.R., and Goodwin S. 1988. Hydrogen concentrations as an indicator of the predominant terminal electron-accepting reactions in aquatic sediments. Geochimica et Cosmochimica Acta., 52: $2993-3003$

Lovley, D.R., Phillips, E.J.P., et al. 1989. "Hydrogen and Formate Oxidation Coupled to Dissimilatory Reduction of Iron or Manganese by Alteromonas putrefaciens." Appl. Environ. Microbiol., 55(3): 700-706.

Loy, A., et al. 2002. Oligonucleotide microarray for 16S rRNA gene-based detection of all recognized lineages of sulfate-reducing prokaryotes in the environment. Applied and Environmental Microbiology, 68: 5064-5081.

Lu, Y. and Pignatello, J.J. 2002. Demonstration of the "Conditioning Effect" in Soil Organic Matter in Support of a Pore Deformation Mechanism for Sorption Hysteresis. Environ. Sci. Technol., 36: 4553-4561.

Luthy, R.G., Aiken, G.A., Brusseau, M.I., Cunningham, S.D., Gschwend, P.M., Pignatello, J.J., Reinhard, M. , Traina, S.J., Weber Jr., W.J. and Westall, J.C. 1997. "Sequestration of Hydrophobic Organic Contaminants by Geosorbents." Environ. Sci. and Technol., 31: 3341-3347.

Lyman W.J., Reehl, W.F., and Rosenblatt, D.H., eds. 1990. Handbook of Chemical Property Estimation Methods. American Chemical Society, Washington, D.C.

Ma, X., and Burken, J.G. 2003. TCE Diffusion to the Atmosphere in Phytoremediation Applications. Envion,. Sci. Technol., 37: 2534-2539.

Mabey, W., and Mill, T. 1978. Critical review of hydrolysis of organic compounds in water under environmental conditions: J. Phys. Chem. Ref. Data, 7: 383-415.

Mader, B.T., Uwe-Goss, K., and Eisenreich, S.J. 1997. "Sorption of Nonionic, Hydrophobic Organic Chemicals to Mineral Surfaces.” Environ. Sci. and Technol., 31: 1079-1086.

Magnuson, J. K., Romine, M. F., Burris, D. R., and Kingsley, M. T. 2000. "Trichloroethene reductive dehalogenase from Dehalococcoides ethenogenes: sequence of tceA and substrate range characterization." Appl. Environ. Microbiol., 66: 5141-5147.

Magnuson, J.K., et al. 1998. Reductive dechlorination of tetrachloroethene to ethene by a two-component enzyme pathway. Applied and Environmental Microbiology, 64: 1270-1275.

Major, D.W., Hodgins, W.W., and Butler, B.J. 1991. Field and laboratory evidence of in situ biotransformation of tetrachloroethene to ethene and ethane at a chemical transfer facility in North Toronto. p. 147-171. In R. E. Hinchee and R. F. Olfenbuttel (eds). On Site Bioreclamation, Butterworth-Heinemann, Boston.

Major, D., McMaster, M. L., et al. 2002. "Field Demonstration of Successful Bioaugmentation to Achieve Dechlorination of Tetrachloroethene to Ethene." Environ. Sci. and Technol., 36(23): 5106-5116. 
Mancini, S.A., Couloume, G.L., et al. 2002. "Hydrogen Isotopic Enrichment: An Indicator of Biodegradation at a Petroleum Hydrocarbon Contaminated Field Site." Environ. Sci. and Technol., 36(11): 2464-2470.

Mann, H.B. and Whitney, D.R. 1947. "On a test of whether one or more random variables is stochastically larger than in the other," Ann. Math. Sciences, 18: 52-54.

March, J. 1985. Advanced Organic Chemistry, 3rd edition: Wiley, New York.

Mars, A. Houwing E.,J., Dolfing J., and Janssen, D.B. 1996. Degradation of toluene and trichloroethylene by Burkholderia cepacia G4 in growth-limited fed-batch culture. Appl. Environ. Microbiol., 62: 886-891.

Maymo-Gatell, X., Tandoi, V., Gossett, J.M., and. Zinder, S. H. 1995. Characterization of an $\mathrm{H}_{2}$-utilizing enrichment culture that reductively dechlorinates tetrachloroethene to vinyl chloride and ethene in the absence of methanogenesis and acetogenesis. Appl. Environ. Microbiol., 61: 3928-3933.

Maymo-Gatell, X., Gossett, J.M., et al. 1997. Dehalococcus Ethenogenes Strain 195: Ethene production from halogenated aliphatics. Battelle: In Situ and On-Site Bioremediation, San Diego, CA, Battelle Press.

Maymo-Gatell, X., Chien, Y.T., Gossett J.M., and Zinder, S.H. 1997. Isolation of a bacterium that reductively dechlorinates tetrachloroethene to ethene. Science, 276: 1568-1571.

Maymo-Gatell, X., Anguish, T., et al. 1999. "Reductive Dechlorination of Chlorinated Ethenes and 1,2Dichloroethane by "Dehalococcoides ethenogenes" 195." Appl. Environ. Microbiol., 65(7): 31083113.

Maymo-Gatell, X., Nijenhuis I., and Zinder, S.H. 2001. Reductive dechlorination of cis-1,2dichloroethene and vinyl chloride by "Dehalococcoides ethenogenes". Environ. Sci. and Technol., 35: 516-521.

McCarthy, K.A., and Johnson, R.L. 1992. Transport of volatile organic compounds across the capillary fringe: Water Resources Research, 29: 1675-1683.

McCarty, P. L., and Reinhard, M. 1993. Biological and chemical transformations of halogenated aliphatic compounds in aquatic and terrestrial environments. In R. S. Oremland (ed). The Biogeochemistry of Global Change: Radioactive Trace Gases, Chapman \& Hall, Inc., New York.

McCarty, P. L., and Semprini, L. 1994. Ground-water treatment for chlorinated solvents. p. 87-116. In R. D. Norris et al. (eds.) Handbook of Bioremediation, Lewis Publishers, Boca Raton.

McCarty, P. L., 1996, Biotic and Abiotic Transformations of Chlorinated Solvents in Ground Water, EPA Symposium on Natural Attenuation of Chlorinated Organics in Ground Water, EPA/540/R96/509, 169 pp.

McCormick, M.L., Bouwer, E.J., and Adriaens, P. 2002. Carbon tetrachloride transformation in a model iron-reducing culture: relative kinetics of biotic and abiotic reactions. Environ. Sci. and Technol., 33(3): 403-410. 
McDonald, M.G. and Harbaugh, A.W. 1988. A Modular Three-Dimensional Finite-Difference GroundWater Flow Model. Techniques of Water-Resources Investigations 06-A1, U.S. Geological Survey Open-File Report 83-875.

Metternicht, G.I. and Zinck, J.A. 2003. Remote sensing of soil salinity: potentials and constraints. Remote Sensing of Environment, Elsevier Science, Ltd. 85: 1-20.

Miller, C.T., Christakos, G., Imhoff, P.T., McBride, J.F., Pedit J.A., Trangenstein, J.A. 1998. Multiphase flow and transport modeling in heterogenous porous media: Challenges and approaches. Adv. Water Resour., 21(2): 77-120.

Miller, C.T., Poirier-McNeill, M.M., Mayer, A.S. 1990. Dissolution of trapped non-aqueous phase liquids: Mass transfer characteristics. Water Resour. Res., 26(11): 2783-2796.

Miller, R.R. 1996. Phytoremediation: Technology Overview Report TO-96-03, Ground Water Remediation Technologies Analysis Center, 22 p.

Millington, R.J., and Quirk, J.P. 1961. Permeability of porous soils: Transactions of Faraday Society, 57: $1200-1207$.

Minnich, M. 1993. Behavior and determination of volatile organic compounds in soil: A literature review: U.S. Environmental Protection Agency EPA/600/R-93/140, 102 p.

Mitsch , W.J. and J. G. Gosselink, 2000, Wetlands, $3^{\text {rd }}$ edition, John Wiley \& Sons, Inc., New York, 920 p.

Morel, F.M.M. and Hering, J.G. 1993. Principles and Applications of Aquatic Chemistry: John Wiley \& Sons, Inc., New York

Morrison, C. and Gannon, F. 1994. The impact of PCR plateau on the quantitative PCR. Biochimica et Biophysica Acta., 1219: 493-498.

Mukherjee, A. 2003. Identification and Natural Attenuation of Trichloroethene and Technitium 99 Along Little Bayou Creek, McCracken County Kentucky. Masters Thesis, Geological Sciences, University of Kentucky, Lexington KY.

Munz, C. and Roberts, P.V. 1987. Air-water phase equilibria of volatile organic solutes: Journal of American Water Works Association, 79: 62-69.

Nam, K, Chung, N., and Alexander, M. 1998. "Relationship Between Organic Matter Content and the Sequestration of Phenanthrene." Environ. Sci. Technol., 32: 3785-3788.

Narayanan, M., Davis, L.C., and Erickson, L.E. 1995. Fate of volatile chlorinated organic-compounds in a laboratory chamber with alfalfa. Env. Sci. and Technol., 29: 2437-2444.

Narayanan, M., Erickson, L.E., and Davis, L.C. 1999. Simple plant-based design strategies for volatile organic pollutants. Env. Prog., 18: 231-242.

Nash, M.S., Atekwana, Estella, and Sauck, W.A. 1997. Geophysical investigation of anomalous conductivity at a hydrocarbon contaminated site. SAGEEP 1997 Proceedings, 675-683.

National Research Council. 1993. In Situ Bioremediation, When Does it Work?: National Academy Press, Washington, D.C., 207 p. 
National Reseach Council, 2001. Natural Attenuation for Groundwater Remediation, National Research Council, Committee on Intrinsic Remediation, National Academy Press, Washington, D.C.

Nellessen, J.E. and Fletcher, J.S. 1993. Assessment of published literature pertaining to the uptake/accumulation, translocation, adhesion and biotransformation of organic chemicals by vascular plants. Environ. Toxicol. Chem., 12: 2045-2052.

Nelson, M.J.K., Montgomery, S.O., and Pritchard, P.H. 1988. Trichloroethylene metabolism by microorganisms that degrade aromatic compounds. Appl. Environ. Microbiol., 54: 604-606.

Neely, W.B. 1985. Hydrolysis, In W.B. Neely and G.E. Blau, editors, Environmental Exposure from Chemicals, Vol. 1: CRC Press, Boca Raton, Florida, p. 157-173.

Neistch, S. L., Arnold, J.G., Kiniry, J.R., Williams, J.R., King, K.W. 2002. Soil and Water Assessment Tool Theoretical Documentation, Version 2000. Grassland, Soil and Water Research Laboratory, U.S. Department of Agriculture Agricultural Research Service, Temple TX.

Newell, C.J., Aziz, C.E., and Cox, G.A. 2001a. "Novel Method to Enhance Chlorinated Solvent Biodegradation by the Use of Barriers," 2001 Int. Containment \& Rem. Technology Conf., June 10-13, 2001, Orlando, Florida.

Newell, C.J., Aziz, C.E., Haas, P.E., Hughes, J. B., and Khan, T.A. 2001b. "Two Novel Methods for Enhancing Source Zone Bioremediation: Direct Hydrogen Addition and Electron Acceptor Diversion", Proceedings of the Battelle Sixth International In Situ and On-Site. Bioremediation Symposium, June 4-7, 2001. San Diego, CA, Battelle Press, Columbus, Ohio, In Press.

Newell, C.J., C.E. Aziz, and G.A. Cox, 2003. "Enhanced Anaerobic Treatment Zones in Groundwater," U.S. Patent 6,562,235; May 13, 2003.

Neumann, A., Wohlfarth, G., and Diekert, G. 1998. Tetrachloroethene dehalogenase from Dehalospirillum multivorans: Cloning, sequencing of the encoding genes, and expression of the pceA Gene in Escherichia coli. Applied and Environmental Microbiology, 180: 4140-4145.

Newman, L.A., Strand, S.E., Choe, N., Duffy, J., Ekuan, G., Ruszaj, M., Shurtleff, B.B., Wilmoth, J. Heilman, P., and Gordon, M.P. 1997. Uptake and biotransformation of trichloroethylene by hybrid poplars. Env. Sci. Tech., 31: 1062-1067.

Newman, L.A., Wang, X., Muiznieks, I.A., Ekuan, G., Ruszaj, M., Cortellucci, R., Domroes, D., Karscig, G., Newman, T., Crampton, R. S., Hashmonay, R. A., Yost, M.G., Heilman, P. E., Duffy, J., Gordon, M. P. and Strand, S.E. 1999. Remediation of Trichloroethylene in an Artificial Aquifer with Trees: A Controlled Field Study, Environ. Sci. and Technol., 33: 2257-2265.

Nichols, T.D., Wolf, D.C., Rogers, H.B., Beyrouty, C.A., and Reynolds, C.M. 1997. Rhizosphere microbial populations in contaminated soils. Water, Air, and Soil Poll, 95: 165-178.

Nietch, C.T., Morris, J.T., and Vroblesky, D.A. 1999. "Biophysical Mechanisms of Trichloroethylene Uptake and Loss in Bald Cypress Growing in Shallow Contaminated Groundwater," Environ. Sci. Technol., 33: 2899-2904.

Norris, T.B., et al. 2002.Soil microbial community structure across a thermal gradient following a geothermal heating event. Applied and Environmental Microbiology, 68: 6300-6309. 
Northcott, G.L., and Jones K.C. 2001. "Partitioning, Extractability, and Formation of Nonextractable PAH Residues in Soil. 2. Effects on Compound Dissolution Behavior." Environ. Sci. Technol., 35: 1111-1117.

Nyquist, J.E., and Corry, C.E. 2002. Self-potential: the ugly-duckling of environmental geophysics. The Leading Edge 21, 446-451.

Nyquist, H. 1928. Certain topics in telegraph transmission theory, Trans. AIEE, 47: 617-644.

Odum, J.M., Tabinowski, J., Lee, M.D., and Fathepure, B.Z. 1995. Anaerobic biodegradation of chlorinated solvents: comparative laboratory study of aquifer microcosms. p. 17-24. In R. D. Norris et al. (eds.) Handbook of Bioremediation, Lewis Publishers, Boca Raton.

Ohkawa, H., Imaishi, H., Shiota, N., Yamada, Y., and Inui, H. 1999. Cytochrome P450s and other xenobiotic metabolizing enzymes in plants; in, Brooks, G.T., and Roberts, T.R., eds., Pesticide Chemistry and Bioscience - The Food Environment Challenge: The Royal Society of Chemistry Special Publication 233, Cambridge, UK, p. 259 - 264.

Oldenburg, G.T., et al. 2003. Oligonucleotide microarray for the study of functional gene diversity in the nitrogen cycle in the environment. Applied and Environmental Microbiology, 69: 1159-1171.

Olsen, G., et al. 1986. Microbial ecology and evolution: a ribosomal rRNA approach. Annual Reviews of Microbiology, 40: 337-365.

Ong, S.K., and Lion, L.W. 1991. Mechanisms for trichloroethylene vapor sorption onto soil minerals: Journal of Environmental Quality, 20: 180 - 188.

Oostrom , M., Hofstee, C., Walker, R.C., and Dane, J.H. 1999. Movement and remediation of trichloroethylene in a saturated heterogeneous porous medium. Journal of Contaminant Hydrology, 37: 159-178.

Orchard, B. J., Doucette, W. J., Chard, J.K., and Bugbee, B. 2000a. "A Novel Laboratory System for Determining Fate of Volatile Organic Compounds in Planted Systems," Environ. Tox. and Chem., 19: $888-894$

Orchard, B. J., Doucette, W. J., Chard, J.K., and Bugbee, B. 2000b. "Uptake of Trichloroethylene by Hybrid Poplar Trees Grown Hydroponically in Flow-Through Plant Growth Chambers," Environ. Tox. and Chem., 19: 895-903

Ouverney, C.C. and Fuhrman J.A., 1999. Combined microautoradiography - 16S rRNA probe technique for determination of radioisotope uptake by specific microbial cell types in situ. Applied and Environmental Microbiology, 65: 1746-1752.

Park, J.K. and Chang, H.N., Microencapsulation of microbial cells. Biotechnology Advances, 2000. 18: 303-319.

Paterson, 1990. Chemosphere 21: 297-331.

Pecher, K., Haderlein, S.B., and Schwarzenbach, R.P. 2002. Reduction of polyhalogneated methanes by surface-bound Fe (II) in aqueous suspensions of iron oxides. Environ. Sci. and Technol. 36(8): 1734-1741. 
Pedit, J.A. and Miller, C.T. 1994. Heterogeneous sorption processes in subsurface systems, 1. Model formulations and applications. Environ. Sci. and Technol., 28(12): 2094-2104.

Phelps, T.J., Malachowsky, K., Schram, R. M., and White, D. C. 1991. Aerobic mineralization of vinyl chloride by a bacterium of the order Actinomycetales. Appl. Environ. Microbiol. 57: 1252-1254.

Pieper, D.H. and Reineke, W. 2000. Engineering bacteria for bioremediation. Current Opinion in Biotechnology, 11:262-270.

Pignatello, J.J., and Xing, B. 1996. "Mechanisms of Slow Desorption of Organic Chemicals to Natural Particles." Environ. Sci. and Technol., 30: 1-11.

Pitts, J.R., Liu, P., Lee, S.H., Tracy, C.E., Smith ,R.D., and Salter, C. 2000. Interfacial stability of thin film hydrogen sensors, Proceedings of the 2000 DOE Hydrogen Program Review, NREL/CP-57028890.

Pivetz, B.E. 2001. Phytoremediation of contaminated soil and ground water at hazardous waste sites: Ground Water Issue, U.S. Environmental Protection Agency EPA/540/S-01/500.

Poulsen, M.M. and Kueper, B.H. 1992. A field experiment to study the behavior of tetrachloroethylene in unsaturated porous media: Environ. Sci. and Technol., 26: 889-895.

Powers, S.E., Loureiro, C.E., Abriola, L.M., Weber Jr., W.J. 1991. Theoretical study of the significance of non-equilibrium dissolution of non-aqueous phase liquids in subsurface systems. Water Resour. Res., 27: 463-477.

Powers, S.E., Abriola, L.M., Weber Jr., W.J. 1994. An experimental investigation for non aqueous phase liquid dissolution in saturated subsurface systems: Transient mass transfer rates. Water Resour. Res., 30(2): 321-332.

Pralle, M.U., Moelders, N., McNeal, M.P., Puscasu, I., Greenwald, A.C., Daly, J.T., and Johnson, E. A. 2002 Photonic Crystal Enhanced Narrow-band Infrared Emitters, Applied Physics Letters, 81(23).

Punshon, T.G. Mills and Adriano, D.C. 2003. Enhanced Monitored Natural Attenuation: Plant Assisted Remediation of the C-Area Burning Rubble Pits (CBRP) - Twin Lakes Wetland Ecosystem. ERDEN-2003-0111.

Puscasu, I. 2003. Photonic Crystals Shrink Infrared Optical Sensors, SPIE OE magazine, May 2003.

Quinn, J.J., Negri, M.C., Hinchman, R.R., Moos, L.P., Wozniak, J.B., Gatliff, E.G. 2001. "Predicting the Effect of Deep-Rooted Hybrid Poplars on the Groundwater Flow System at a Large-Scale Phytoremediation Site," Int. J. Phytoremediation, 3: 41-60

Radajewski, S., et al. 2000. Stable-isotope probing as a tool in microbial ecology. Nature, 403: 646-649.

Raeymaekers, L.A. 1995commentary on the practical applications of quantitative PCR. Genomes Research, 5: 91-94.

Reinhard, M., Curtis, G.P., and Kriegman, M.R. 1990. Abiotic reductive dechlorination of carbon tetrachloride and hexachloroethane by environmental reductants: Project Summary, EPA/600/S290/040, September 1990. 
Reinhard, M. Cunningham, J.A., and Hoelen, T.P., 2001. "Enhancing Reductive Dehalogenation of Chlorinated Ethylenes Under Sulfate-Reducing Conditions", Abstract from Battelle Sixth International In Situ and On-Site. Bioremediation Symposium. June 4-7, 2001. San Diego, CA

Rice, D.W., Grose, R.D., Michaelsen, J.C., Dooher, B.P., MacQueen, D.H., Cullen, S.J., Kastenberg, W.E., Everett, L.G. and Marino, M.A. 1995. California Leaking Underground Storage Tank Historical Case Analysis, report submitted into record, California State Water Resources Control Board Underground Storage Tank Program and the Senate Bill 1764 Leaking Underground Fuel Tank Advisory Committee, California Environmental Protection Department, Sacramento CA, $20 \mathrm{pp}$.

Richardson, R.E., et al. 2002. Phylogenetic characterization of microbial communities that reductively dechlorinate TCE based upon a combination of molecular techniques. Environ. Sci. and Technol., 36: $2652-2662$.

Riley, R.G., and Zachara J.M. 1992. Chemical Contaminants on DOE Lands and Selection of Contaminant Mixtures for Subsurface Science Research. DOE/ER-0547T, U.S. Department of Energy, Office of Energy Research, Subsurface Science Program, Washington, D.C.

Rondon, M.R., et al. 2000. Cloning the soil metagenome: a strategy for accessing the genetic and functional diversity of uncultured microorganisms. Applied and Environmental Microbiology, 66: 2541-2547.

Rosner, B.M., McCarty P.L., and Spormann A.M. 1997. In vitro studies on reductive vinyl chloride dehalogenation by an anaerobic mixed culture. Applied and Environmental Microbiology, 63: 4139-4144.

RTDF. 1997. Natural Attenuation of Chlorinated Solvents in Groundwater: Principles and Practices. Remediation Technology Development Forum.

Rugner, H., S. Kleineidam, and Grathwohl, P. 1999. Long term sorption kinetics of phenathrene in aquifer materials. Environ. Sci. and Technol., 33(10):1645-1651.

Rumer, R. R. and Mitchell, J. K. 1995. Assessment of Barrier Containment Technologies: A Comprehensive Treatment for Environmental Remediation Applications, Publication No. PB96180583, A publication prepared by the U.S. DOE, the U. S. EPA and the Dupont Company; available from the National Technical Information Service, Springfield VA.

Runkel, R.L. 1998. One-dimensional transport with inflow and storage (OTIS): A solute transport model for streams and rivers. U.S. Geological Survey Water Resources Investigation Report no. 98-4018, $73 \mathrm{p}$.

Ryan, J.A. 1988. Chemosphere, 17: 2299-2323.

Sandermann Jr., H. 1994. Higher plant metabolism of xenobiotics: the 'green liver' concept: Pharmacogenetics, 4: $225-241$.

Sanford, R.A., et al. 1996. Characterization of Desulfitobacterium chlororespirans sp. nov., which grows by coupling the oxidation of lactate to the reductive dechlorination of 3-chloro-4hydroxybenzoate. Applied and Environmental Microbiology, 62: 3800-3808. 
Sato, M., and Mooney, H.M. 1960, The electrochemical mechanism of sulfide self potentials. Geophysics, 25: 226-249.

Sauck, W. 1998. A conceptual model for the geoelectrical response of LNAPL plumes in granular sediments. SAGEEP 1998 Proceedings, 805-817.

Sauck, W. 2000. A model for the resistivity structure of LNAPL plumes and their environs in sand sediments. J. Appl. Geophysics, 44: 151-66.

Schlebaum, W., Schraa, G., and Wvan, Riemsdijk.H. 1999. "Influence of Nonlinear Sorption Kinetics on the Slow-Desorbing Organic Contaminant Fraction in Soil." Environ. Sci. and Technol., 33: 1413-1417.

Schnabel, W.E., Dietz , A.C., Burken, J.G., Schnoor, J.L., and Alvarez, P.D.. 1997. Uptake and transformation of trichloroethylene by edible garden plants. Wat. Res., 4: 816-824.

Schnoor, J.L., Licht, L.A., McCutcheson, S.C., Wolfe, N.L., and Carreira, L.H. 1995. Phytoremediation of organic and nutrient contaminants. Environ. Sci. and Technol., 29: 318-323.

Schnoor, J.L. 1997. Phytoremediation. Technology Evaluation Report TE 98-01, Ground-water Remediation Technologies Analysis Center.

Schnoor, J.L. 2002. Phytoremediation of soil and groundwater. Technology Evaluation Report TE-02-01, Groundwater Remediation Technologies Analysis Center, 52 p.

Schwarzenbach, R.P. and Westall, J. 1981. Transport of nonpolar organic compounds from surface water to ground water: Laboratory sorption studies. Environ. Sci. and Technol., 15(11): 11-18.

Schwartzenbach, R.P., Gschwend, P.M., and Imboden, D.M. 1993. Environmental Organic Chemistry: John Wiley \& Sons, Inc., New York, 681p.

Semprini, L., Roberts, P.V., Hopkins, G.D., and McCarty, P.L. 1990. A field evaluation of in situ biodegradation of chlorinated ethenes. Part 2. Results of biostimulation and biotransformation experiments. Ground Water, 28: 715-727.

Semprini, L., Hopkins G.D., Roberts, P.V., Grbic-Galic, D., and McCarty, P.L. 1991. A field evaluation of in situ biodegradation of chlorinated ethenes. Part 3. Studies of competitive inhibition. Ground Water, 29: 239-250.

Semprini, L. 1995. In situ bioremediation of chlorinated solvents. Environ. Health Perspec., 103: 101105.

Semprini, L. 1997. Strategies for the aerobic co-metabolism of chlorinated solvents. Current Opinion in Biotechnology, 8: 296-308.

Shang, T.Q., Doty, S.L., Wilson, A.M., Howald, W.N., and Gordon, M.P. 2001. Trichloroethylene oxidative metabolism in plants: the trichloroethanol pathway. Phytochem., 58: 1055-1065.

Shann, J.R. 1995. The role of plants and plant/microbial systems in the reduction exposure. . Environ. Health. Perspect. 103(Supplement 5): 13-15.

Shannon, C.E. 1949. Communication in the presence of noise, Proc. Institute of Radio Engineers, 37(1): 10-21. 
Sharma, P.K., and McCarty, P.L. 1996. Isolation and characterization of a facultatively aerobic bacterium that reductively dehalogenates tetrachloroethene to cis-1,2-dichloroethene. Appl. Environ. Microbiol., 62: 761-765.

Sherwood-Lollar, B., Slater, G.F., et al. 2001. "Stable Carbon Isotope Evidence for Intrinsic Bioremediation of Tetrachloroethene and Trichloroethene at Area 6, Dover Air Force Base." Environ. Sci. and Technol., 35(2): 261-269.

Shevnin, V., Mousatov, A., Nakamura-Labastida, E., Delgado-Rodriquez, O., Mejia-Aguilar, A., Sanchez-Osio, J., Sanchez-Osio, H. 2003. Study of oil pollution in airports with resistivity sounding. SAGEEP 2003 Proceedings, 180-189.

Shimp, J.F., Tracy J.C., Davis L.C., Lee E., Huang W., Erickson L.E., and Schnoor J.L. 1993. Beneficial effects of plants in the remediation of soil and groundwater contaminated with organic materials. Crit. Rev. Environ. Sci. and Technol., 23: 41-77.

Shimp, J.F., Tracy, J.C., Davis, L.C., Lee, E., Huang, W., Erickson,, and Schnoor, J.L. 1993. Beneficial effects of plants in the remediation of soil L. E. and groundwater contaminated with organic materials. Environ. Sci. and Technol., 23: 41-77.

Shoemaker, C. Willis, M., Zhang, W., Gossett, J. 2001. "Model Analysis of Reductive Dechlorination With Data From Cape Canaveral Field Site" Abstract from Battelle Sixth International In Situ and On-Site. Bioremediation Symposium. June 4-7, 2001. San Diego, CA.

Simmons, C.T., Fenstemaker, T.R., and Sharp, Jr., J.M. 2001. Variable-density groundwater flow and solute transport in heterogeneous porous media: Approaches, resolutions and future challenges. Journal of Contaminant Hydrology, 52: 245-275.

Sinke A.J.C. 1998. A Decision Support System for Acceptance of Natural Attenuation as Remediation Strategy. NOBIS, Gouda. (The Dutch Research Programme Biotechnological In Situ Remediation (Dutch acronym: NOBIS))

Slater, G.F., Sherwood-Lollar, B. et al. 2001. "Variability in Carbon Isotope Fractionation during Biodegradation of Chlorinated Ethenes: Implications for Field Applications." Environ. Sci. and Technol., 35(5): 901-907.

Slater, G.F., et al. 2001. Variability in carbon isotopic fractionation during biodegradation of chlorinated ethenes: Implications for field applications. Environ. Sci. and Technol., 35: 901-907.

Sleep, B.E., and Sykes, J.F. 1989. Modeling the transport of volatile organics in variably saturated media: Water Resources Research, 25: 81-92.

Small, J., et al. 2001. Direct detection of 16S rRNA in soil extracts by using oligonucleotide microarrays. Applied and Environmental Microbiology, 67: 4708-4716.

Smidt, H., et al. 2000. Halorespiring bacteria - molecular characterization and detection. Enzyme and Microbial Technology, 27: 812-820.

Smith, J.H., Bomberg, D.C., and Haynes, D.L. 1980. Prediction of volatilization rates of high-volatility chemicals from natural water bodies. Environ. Sci. and Technol., 14: 1332-1337. 
Song, D. L., M. E. Conrad, et al. 2002. "Stable Carbon Isotope Fractionation during Enhanced In Situ Bioremediation of Trichloroethene." Environ. Sci and Technol., 36(10): 2262-2268.

Sophocleous, M.A., Townsend, M.A., Vogler, L.D., McLain, T.J., Marks, E.T., and Coble, G.R. 1988. Experimental studies in stream-aquifer interaction along the Arkansas River in central Kansas: Field testing and analysis. Journal of Hydrology, 98: 249-273.

Sophocleous, M.A., Koussis, A.D., Martin, J.L., and Perkins, S.P. 1995. Evaluation of simplified streamaquifer depletion models for water rights administration. Ground Water, 33:579-588.

Sophocleous, M.A. 2002. Interactions between groundwater and surface water: the state of the science. Hydrogeology Journal, 10: 52-67.

Spencer, W.F., and Cliath 1970. Resorption of lindane from soil as related to vapor density: Soil Science Society of America Proceedings, 34: 547 - 578.

Spiro, A., Lowe, M., and Brown, D. 2000. A bead-based method for multiplexed identification and quantitation of DNA sequences using flow cytometry. Applied and Environmental Microbiology, 66: 4258-4265.

Spiro, A. and Lowe, M. 2002. Quantitation of DNA sequences in environmental PCR products by a multiplexed, bead-based method. Applied and Environmental Microbiology, 68: 1010-1013.

Steiner, H., Jakusch, M., Kraft, M., Karlowatz, M., Baumann, T., Niessner, R., Konz, W., Brandenburg, A., Michel, K., Boussard-Pledel, C., Bureau, B., Lucas, J., Reichlin, Y., Katzir, A., Fleischmann, N., Staubmann, K., Allabashi, R., Bayona, J. M., Mizaikoff, B. 2003. In Situ Sensing of Volatile Organic Compounds in Groundwater:First Field Tests of a Mid-Infrared Fiber Optic Sensing System, Appl. Spectroscopy, 57: 607-613.

Stephanatos B.N., Walters, K., Funk, A., and MacGregor, A. 1991. Pitfalls associated with the assumption of a constant partition coefficient in modeling sorbing solute transport through the subsurface. Proceedings of Internal Ground Water Symposium, Nashville, Tennessee, p. 13-20.

Sternberg, B.K., and Oehler, D.Z. 1990. Induced polarization hydrocarbon surveys: Arkoma Basin case histories. In Induced polarization: applications and case histories, SEG Investigations in Geophysics, 4: 354-378.

Stettler, R.F., Koster, R., and Steenackers, V. 1980. Interspecific crossability studies in poplars. Theor. Appl. Genet., 58 :273-282.

Stevens, P.W., Henry, M.R., and Kelso, D.M. 1999. DNA hybridization on microparticles: determining capture-probe density and equilibrium dissociation constants. Nucleic Acid Research, 27: 17191727.

Stomp, A.M., Han, K.H., Wilbert, S., and Gordon, M.P. 1993. Genetic improvement of tree species for remediation of hazardous wastes. In vitro Cell Dev. Biol. 29: 227-232.

Stumm, W., and Morgan, J.J. 1981. Aquatic Chemistry: John Wiley \& Sons, New York.

Sun, B., et al. 2002. Microbial dehalorespiration with 1,1,1-trichloroethane. Science, 298: 1023-1025. 
Suzuki, M.T. and Giovannoni, S.J. 1996. Bias caused by template annealing in the amplification of mixtures of 16S rRNA genes by PCR. Applied and Environmental Microbiology, 62: 625-630.

Tandoi, V., et al. 1994. Reductive dehalogenation of chlorinated ethenes and halogenated ethanes by a high-rate anaerobic enrichment culture. Environ. Sci. and Technol., 28: 973-979.

Tepfer, D. 1989. Ri T-DNA from Agrobacterium rhizogenes: a source of genes having applications in rhizosphere biology and plant development, ecology and evolution. In: T. Kosuge and E.W. Nester, eds. Plant-microbe interactions, molecular and genetic perspectives, 3: 294-342.

Thibaus-Erkey, Guo, Y., Erkey, C., and Akgerman, A.1996. "Mathematical Modeling of Adsorption And Desorption of Volatile Contaminants From Soil: Influence of Isotherm Shape on Adsorption and Desorption Profiles." Environ. Sci. and Technol., 30: 2127-2134.

Thurman D.A., and Rankin, A.J. 1982. The role of organic acids in zinc tolerance in Deschampsia caespitosa. New Phytol., 91: 629-635.

Torsvik, V., Goksoyr, J., and Daae, F.L., 1990. High diversity of DNA of soil bacteria. Applied and Environmental Microbiology, 56: 782-787.

Torsvik, V., et al., 1998. Novel techniques for analysing microbial diversity in natural and perturbed environments. Journal of Biotechnology, 64: 53-62.

Trapp, S., and McFarlane, J.C., eds., 1994, Plant contamination - modeling and simulation of organic chemical processes: Lewis Publishers, Boca Raton.

Tschaplinski, T.J., and Blake, T.J. 1989a. Correlation between early root production, carbohydrate metabolism, and biomass production in hybrid poplar. Can. J. Bot., 67: 2168-2174.

Tschaplinski, T.J., and Blake, T.J. 1989b. Water stress tolerance and late-season organic solute accumulation in hybrid poplar. Can. J. Bot., 67: 1681-1689.

Tschaplinski, T.J., and Blake, T.J. 1994. Carbohydrate mobilization following shoot defoliation and decapitation in hybrid poplar. Tree Physiol. 14: 141-151.

Tschaplinski, T.J., and Tuskan, G.A. 1994. Water-stress tolerance of black cottonwood and eastern cottonwood clones and four of their hybrid progeny. II. Metabolites and inorganic ions that constitute osmotic adjustment. Can. J. For. Res., 24: 681-687.

Tuskan, G.A. 1998. Short-rotation woody crop supply systems: We do we know and what do we need to know? Biomass and Bioenergy, 14: 307-315.

US Department of Defense (US DOD) 1994. Defense Environmental Cleanup Program Annual Report to Congress for Fiscal Year 1993. Office of the Assistant Deputy Undersecretary of Defense for Environmental Security/Cleanup, Washington, D.C.

US Department of Energy (US DOE) 2002a. Technical Assistance to Kansas City Plant: Mitigation of Polychlorinated Biphenyl Discharges, WSRC-RP-2003-00276, US DOE Office of Environmental Management, available from the US DOE Office of Science and Technology Information, Oak Ridge TN. 
US Department of Energy (US DOE) 2002b. Alternative Project Plan for Technology Development, Accelerated Risk Reduction Through Innovative Remediation of Chlorinated Ethenes Using Monitored Natural Attenuation. US DOE Office of Environmental Management.

US Environmental Protection Agency (US EPA) 1989. Methods for evaluating attainment of cleanup standards, volume 1, Soils and solid media: EPA/230/02-89-042.

US Environmental Protection Agency (US EPA) 1990. National Priorities List Sites. EPA/540/4-90, Volumes 002 to 052.

US Environmental Protection Agency (US EPA) 1992. Methods for evaluating attainment of cleanup standards, volume 2, Groundwater: EPA/230-R-92-014.

US Environmental Protection Agency (US EPA) 1993. Simplified Method Program - Variable Complexity Stream Toxics Model (SMPTOX3). U. S. EPA, Region IV, Atlanta GA. Version 2.01, January, 1993.

US Environmental Protection Agency (US EPA) 1998. Technical protocol for evaluating natural attenuation of chlorinated solvents in ground water, EPA/600/R-98/128: Office of Research and Development, U.S. Environmental Protection Agency, Washington, D.C., p. 232.

US Environmental Protection Agency (US EPA) 1999. Monitored Natural Attenuation of Chlorinated Solvents: U.S. EPA Remedial Technology Fact Sheet, EPA/600/F-98/022, U.S. Environmental Protection Agency, Office of Research and Development, National Risk Management Research Laboratory, Ada OK, May 1999.

US Environmental Protection Agency (US EPA) 1999a. Use of Monitored Natural Attenuation at Superfund, RCRA Corrective Action, and Underground Storage Tank Sites, Final, Office of Solid Waste and Emergency Response. April 21. Directive Number 9200.4-17P.

US Environmental Protection Agency (US EPA), 1999b, Use of Monitored Natural Attenuation at Superfund, RCRA Corrective Action, and Underground Storage Tank Sites, Draft Interim Final :U.S. EPA Office of Solid Waste and Emergency Response Directive 9200.4-17 (http://www.epa.gov/OUST/directiv/9200417z.htm)

US Environmental Protection Agency (US EPA) 1999. Phytoremediation Resource Guide: U. S. Environmental Protection Agency, Solid Waste and Emergency Response, EPA 542-B-99-003, p. 30.

US Environmental Protection Agency (US EPA) 2000. Proceedings of the Ground-water/surface-water interactions workshop. EPA/542/R-00/007.

US Environmental Protection Agency (US EPA) 2001. AQUATOX - A Modular Fate and Effects Model for Aquatic Ecosystems - Release 1.1: Volume 2 - Technical Documentation. EPA-823-R-01-007, U.S. EPA, Office of Water, Washington D.C., November, 2001.

US Environmental Protection Agency (US EPA) 2002 Draft. Performance Monitoring for Natural Attenuation Remedies in Groundwater.

Vanhala, H. 1997. Mapping oil contaminated sands and tills with the spectral induced polarization (SIP) method. Geophysical Prospecting, 45: 303-326. 
Verce, M.F., Ulrich, R.L., and Freedman, D.L. 2000. Characterization of an isolate that uses vinyl chloride as a growth substrate under aerobic conditions. Applied and Environmental Microbiology, 66: 3535-3542.

Vogel, T.M., and McCarty, P.L.. 1985. Biotransformation of tetrachloroethylene to trichloroethylene, dichloroethylene, vinyl chloride, and carbon dioxide under methanogenic conditions. Appl. Environ. Microbiol., 49: 1080-1083.

Vogel, T.M., and Reinhard, M. 1986. Reaction products and rates of disappearance of simple bromoalkanes, 1,2-dibromopropane and 1,2-dibromoethane in water: Environ. Sci. and Technol., 20(10): 992-997.

Vogel, T.M., Criddle, C.S., and McCarty, P.L. 1987. Transformation of halogenated aliphatic compounds. Environ. Sci. and Technol., 21: 722-736.

Vogel, T.M., and McCarty, P.L. 1987. Abiotic and biotic transformations of 1,1,1-trichloroethane under methanogenic conditions: Environ. Sci. and Technol. 21(12): 1208-1213.

Vogel, T.M., Criddle, C.S., and McCarty, P. L. 1987. Transformations of halogenated aliphatic compounds: Environ. Sci. and Technol., 21(8): 722-736.

Vogel, T.M. 1994. Natural Bioremediation of Chlorinated Solvents, In: Handbook of Bioremediation. Lewis Publishers, Boca Raton, FL. 1994.

Vogel, T.M. 1994. Natural bioremediation of chlorinated solvents. p. 201-225. In R. D. Norris et al. (eds.) Handbook of Bioremediation, Lewis Publishers, Boca Raton.

Vroblesky, D.A., Nietch, C.T., and Morris, J.T. 1999. Chlorinated ethenes from groundwater in tree trunks. Env. Sci. and Technology, 33:510-515.

Wallner, G., et al. 1997. Flow sorting of microorganisms for molecular analysis. Applied and Environmental Microbiology, 63: 4223-4231.

Walton, B.T. and Anderson, T.A. 1990. Microbial degradation of trichloroethylene in the rhizosphere: Potential application to biological remediation of waste sites. Appl. Environ. Microbiol., 56: 1012-1016.

Wang, T.C., and Tan, C.K. 1990. Reduction of halogenated hydrocarbons with magnesium hydrolysis process: Bull. Environ. Contam. Toxicol., 45: 149-156.

Ward, D.M., et al., 1992. Ribosomal RNA analysis of microorganisms as they occur in nature. Advances in Microbial Ecology, 12: 219-286.

Washington, J. B., Pignatello, J.J., Lu, Y., Rivikovitch, P.I., Neimark, A.V., and Xing, B. 2003. Sorption Hysteresis of Benzene in Charcoal Particles. Environ. Sci. Technol., 37: 409-417.

Washington, J.W. 1995. Hydrolysis rates of dissolved volatile organic compounds: principles, temperature effects and literature review. Ground Water, 33: 415-424.

Weaver, J.W., Wilson, J.T., and Kampbell, D.H. 1995. Natural bioattenuation of trichloroethene at the St. Joseph, Michigan superfund site. EPA Project Summary EPA/600/SV-95/001. USEPA, Washington D.C. 
Weaver, J.W., Wilson, J.T., and Kampbell, D.H. 1996. Extraction of Degradation Rate Constants from the St. Joseph, Michigan, Trichloroethene Site. Symposium on Natural Attenuation of Chlorinated Organics in Ground Water, p. 69-73. EPA/540/R-96-509.

Weber Jr., W.J., and Wang, H. 1996. "A Distributed Reactivity Model for Sorption by Soils and Sediments. 4. Intraparticle Heterogeneity and Phase-Distribution Relationships Under NonEquilibrium Conditions." Environ. Sci and Technol., 30: 881-888.

Weber Jr., W.J., Kim, S.H., and Johnson, M.D. 2002. Distributed Reactivity Model for Sorption by Soils and Sediments. 15. High-Concentration Co-Contaminant Effects on Phenanthrene Sorption and Desorption. Environ. Sci. and Technol., 36: 3625-3634.

Werkema, D.D., 2002, Geoelectrical response of an aged LNAPL plume: implications of monitoring natural attenuation. $\mathrm{PhD}$ thesis, Western Michigan University, $136 \mathrm{pp}$.

Werth, C.J. and Reinhard, M. 1997. Effects of Temperature on Trichloroethylene Desorption from Silica Gel and Natural Sediments. 2. Kinetics. Environ. Sci. and Technol., 31: 697-703.

Werth, C.J., McMillan, S.A., and Castilla, H.J. 2000. Structural evaluation of slow desorbing sites in model and natural solids using temperature stepped desorption profiles. 1. Model development. Environ. Sci. Technol., 34(14): 2959-2965.

Westinghouse Savannah River Company, 1999. Characterization Report to Support Phytoremediation Efforts for the Southern Sector, Savannah River Site, Aiken, South Carolina. WSRC-TR-9900113, Revision 0, April, Westinghouse Savannah River Company. Aiken, SC

Westinghouse Savannah River Company, 2000a. Phytoremediation of Trichloroethylene and Perchloroethylene in the Southern Sector of SRS: Interim Report, WSRC-TR-2000-00372, Revision 0, December, Westinghouse Savannah River Company. Aiken, SC

Westinghouse Savannah River Company, 2000b. Characterization Activities to Evaluate Chlorinated Solvent Discharges to Tims Branch from the A/M Area of the Savannah River Site, WSRC-TR2000-00472, November 2000, Westinghouse Savannah River Company. Aiken, SC

Westinghouse Savannah River Company, 2001. FY01 Phytoremediation of Chlorinated Ethenes in Southern Sector Seepline Sediments of Savannah River Site. WSRC-TR-2001-00437 Westinghouse Savannah River Company. Aiken, SC

Westinghouse Savannah River Company, 2002. FY02 Final Report on Phytoremediation of Chlorinated Ethenes in Southern Sector Seepline Sediments of the Savannah River Site. WSRC-TR-200200557 Westinghouse Savannah River Company. Aiken, SC.

Westinghouse Savannah River Company, 2003. Scientific Basis for Monitored Natural Attenuation and Enhanced Passive Remediation for Chlorinated Solvents - DOE Alternatie Project for Technology Acceleration Implementation Plan. WSRC-RP-2003-00286. Westinghouse Savannah River Company, Aiken, SC

White, D.C. and Ringelberg, D.B. 1998. Signature lipid biomarker analysis, in Techniques in Microbial Ecology, R.S. Burlage, et al., Editors., Oxford University Press: New York. p. 255-272. 
Wiedemeier, T.H. and M Barden, 2002, Natural Attenuation for Remediation of Contaminated Sites, National Groundwater Association Course Notes.

Wiedemeier, T.H., Wilson, J.T., Kampbell, D.H., Miller, R.N., and Hansen, J.E., 1995, Technical protocol for implementing intrinsic remediation with long-term monitoring for natural attenuation of fuel contamination dissolved in groundwater: US Air Force Center for Environmental Excellence, San Antonio, Texas.

Wiedemeier, T.H., Swanson, M.A., Moutoux, D.E., Wilson, J.T., Kampbell, D.H., Hansen, J.E., and Haas, P., 1996a, Overview of the Technical Protocol for Natural Attenuation of Chlorinated Aliphatic Hydrocarbons under Development for the U.S. Air Force Center for Environmental Excellence: EPA/540/R-96/509, p. 35-59.

Wiedemeier, T.H., Swanson, M.A., Wilson, J.T., Kampbell, D.H., Miller, R.N., and Hansen, J.E. 1996b. Approximation of biodegradation rate constants for monoaromatic hydrocarbons (BTEX) in groundwater: Groundwater Monitoring and Remediation, v. 16, no. 3, Summer 1996, p. 186-194.

Wiedemeier, T.H., and Chapelle, F.H. 1997. Technical Guidelines for Evaluating Monitored Natural Attenuation at Navy and Marine Corps Facilities - Draft: US Navy Document

Wiedemeier, T.H., Swanson, M.A., Moutoux, D.E., Gordon, E.K., Wilson, J.T., Wilson, B.H., Kampbell, D.H., Hansen, J.E., Haas, P., and Chapelle, F.H. 1998. Technical Protocol for Evaluating the Natural Attenuation of Chlorinated Solvents Dissolved in Groundwater, Air Force Center for Environmental Excellence, San Antonio, Texas.

Wiedemeier, T. H., M.A. Swanson, D.E. Moutoux, E.K. Gordon, J.T. Wilson, B.H. Wilson, D.H. Kampbell, J.E. Hansen, and P. Haas., 1998. "Technical Protocol for Evaluation Natural Attenuation of Chlorinated Solvents in Groundwater," EPA/600/R-98/128.

Wiedemeier, T.H., Rifai, H.S., Newell, C.J., and Wilson, J.T. 1999. Natural Attenuation of Fuel Hydrocarbons and Chlorinated Solvents, John Wiley and Sons, New York, New York.

Williams, J.B. 2002. Phytoremediation in wetland ecosystems: Progress, problems, and potential. Crit. Rev. Plant Sci., 21:607-635.

Wilson, J.T., and Wilson, B.H. 1985. Biotransformation of trichloroethylene in soil. Appl. Environ. Microbiol., 49: 242-243.

Wilson, J.T., Kampbell, D.H., Weaver J.W., Imbrigiotta T., and Ehlke T. 1995. A review of intrinsic bioremediation of trichloroethylene in ground water at Picatinny Arsenal, New Jersey, and St. Joseph, Michigan. Symposium on Bioremediation of Hazardous Wastes: Research, Development, and Field Evaluations. EPA, Rye Brook, New York.

Winter, T.C. 1999. Relations of streams, lakes, and wetlands to groundwater flow systems. Hydrogeology Journal 7: 28-45.

Winter, T.C. 2000. Interaction of ground water and surface water pages 15-20 In. Proceedings of the Ground-water/surface-water interactions workshop. EPA/542/R-00/007. 
Wintzingerode, F.V., Gobel, U.B., and Stackebrandt, E. 1997. Determination of microbial diversity in environmental samples: pitfalls of PCR-based rRNA analysis. FEMS Microbiology Reviews, 21: 213-229.

Wintzingerode, F.V., et al. 2001. Development of primers for amplifying genes encoding CprA- and PceA-like reductive dehalogenases in anerobic microbial consortia, dechlorinating trichlorobenzene and 1,2-dichloropropane. FEMS Microbiology Ecology, 35: 189-196.

Wu, W.-M., Nye, J., Hickey, R.F., Jain, M.K., and Zeikus, J.G. 1995. Dechlorination of PCE and TCE to ethene using an anaerobic microbial consortium. p. 45-52. In R. D. Norris et al. (eds.) Handbook of Bioremediation, Lewis Publishers, Boca Raton.

Yang, Y. and McCarty, P.L. 1998. "Competition for Hydrogen within a Chlorinated Solvent Dehalogenating Anaerobic Mixed Culture." Environ. Sci. and Technol. 32(22): 3591-3597.

Yang, Y., and McCarty, P. 2001. "Sulfate Impact on Anaerobic Reductive Dehalogenation", Abstract from Battelle Sixth International In Situ and On-Site. Bioremediation Symposium. June 4-7, 2001. San Diego, CA

Yotsukura, N., Stedfast, D.A., and Brutseart, W.H. 1983. An assessment of steady state propane gas method for reaeration coefficients-Cowaselon Creek, New York. U.S. Geological Survey Water Resources Investigation Report 83-4183, 95 p.

Young, S.C., Julian H.E., Pearson H.S., Molz F.J., and Bowman G.K., Application of the Electromagnetic Borehole Flowmeter, EPA/600/SR-98/058.

Zhang, C.L. 2002. Stable carbon isotopes of lipid biomarkers: analysis of metabolites and metabolic fates of environmental microorganisms. Current Opinion in Biotechnology, 13: 25-30.

Zheng, C. and Wang, P.P. 1999. MT3D: A Modular Three-Dimensional Multispecies Transport ModelDocumentation and User's Guide. http://hydro.geo.ua.edu/mt3d.

Zhou, J. and Thompson, D.K. 2002. Challenges in applying microarrays to environmental studies. Current Opinion in Microbiology, 13: 204-207. 
WSRC-TR-2003-00328

February 19, 2004

9.0 Appendices Large Prioritization Tables 


\section{Most Promising Characterization and Monitoring Science and Technology Targets}

KEY:

R\&D Timeframe: $\mathrm{N}$ (near-term) $<2$ years, $\mathrm{M}$ (medium-term) $2-5$ years, $\mathrm{L}$ (long-term) $>5$ years.

Cost to impact need. $\$=\leq \$ 100 \mathrm{~K}, \$ \$=>\$ 100 \mathrm{~K}-\$ 500 \mathrm{~K}, \$ \$ \$=>\$ 500 \mathrm{~K}$

Overall Ranking: High (H), Medium (M) or Low (L)

\begin{tabular}{|c|c|c|c|c|c|c|c|c|}
\hline \multirow[t]{2}{*}{ Technical Concept or Objective } & \multirow[t]{2}{*}{ Pros } & \multirow[t]{2}{*}{ Cons } & \multirow[t]{2}{*}{ R\&D Timeframe } & \multirow[t]{2}{*}{ cost } & \multicolumn{3}{|c|}{ Category } & \multirow[t]{2}{*}{ Overall Ranking } \\
\hline & & & & & Policy & Technical & Implementation & \\
\hline $\begin{array}{l}\text { Advanced Bioassessment Tools } \\
\text { Develop methods or protocols for determining } \\
\text { ongoing microbial processes at different sites, } \\
\text { including reductive processes (Dehalococcoides } \\
\text { etc.) } \\
\text { a. new methods to quantify growth. } \\
\text { b. gene sequences used to develop molecular } \\
\text { probes for micro arrays and quantitative } \\
\text { PCR. } \\
\text { Identify additional key functional genes for use in } \\
\text { Microarrays } \\
\text { - genes whose expression correlates with } \\
\text { specific environmental conditions } \\
\text { - DNA microarrays used to simultaneously } \\
\text { monitor the expression of many genes }\end{array}$ & $\begin{array}{l}\text { - Direct measurement may } \\
\text { ultimately replace several indirect } \\
\text { measures. } \\
\text { - Reaction rates may correlate to } \\
\text { better measurement and results } \\
\text { provide better model input. } \\
\text { - May make explanation of } \\
\text { MNA/EPR simpler. } \\
\text { - Exploits rapid growth in this } \\
\text { discipline and progress since EPA } \\
\text { protocol in 1998. } \\
\text { - Future costs reductions possible if } \\
\text { analogy to medical diagnostics is } \\
\text { valid. } \\
\text { - Technology (depending on } \\
\text { specific tool) can provide } \\
\text { information about the presence, } \\
\text { expression and rate of microbial } \\
\text { treatment processes. }\end{array}$ & $\begin{array}{l}\text { - Success relies on costs coming } \\
\text { down significantly from current } \\
\text { levels. } \\
\text { - Some solid research is needed } \\
\text { to validate approach. } \\
\text { - Possibility for interferences } \\
\text { under a wide range of } \\
\text { conditions. }\end{array}$ & $\mathrm{N}$ to $\mathrm{M}$ & $\$ \$$ & $\mathrm{M}$ & $\begin{array}{c}\mathrm{N} \text { to } \mathrm{M} \\
\text { High priority }\end{array}$ & $\mathrm{M}$ & $\begin{array}{l}\text { H } \\
\text { As a class, the } \\
\text { bioassessment tools } \\
\text { were the highest } \\
\text { rated in the } \\
\text { technical target list }\end{array}$ \\
\hline $\begin{array}{l}\text { Develop correlations between species, functional } \\
\text { genes, and degradation rate and potential } \\
\text { - Laboratory and field tests to develop and } \\
\text { correlate parameters } \\
\text { - Assess factors that would bias the } \\
\text { application of these molecular tools }\end{array}$ & $\begin{array}{l}\text { - See above. } \\
\text { - These data would provide a key } \\
\text { part of the validation of the validity } \\
\text { and usefulness of the measurements }\end{array}$ & See above & $\mathrm{N}$ to $\mathrm{M}$ & $\$ \$$ & $\mathrm{M}$ & $\begin{array}{c}\mathrm{N} \text { to } \mathrm{M} \\
\text { High priority }\end{array}$ & $\mathrm{M}$ & $\mathbf{H}$ \\
\hline
\end{tabular}




\begin{tabular}{|c|c|c|c|c|c|c|c|c|}
\hline \multirow[t]{2}{*}{ Technical Concept or Objective } & \multirow[t]{2}{*}{ Pros } & \multirow[t]{2}{*}{ Cons } & \multirow[t]{2}{*}{ R\&D Timeframe } & \multirow[t]{2}{*}{ cost } & \multicolumn{3}{|c|}{ Category } & \multirow[t]{2}{*}{ Overall Ranking } \\
\hline & & & & & Policy & Technical & Implementation & \\
\hline $\begin{array}{l}\text { Further research on oxidative and reductive } \\
\text { processes; } \\
\text { - Basic research to investigate possible } \\
\text { microorganisms other than Dehalococcoides-like } \\
\text { microorganisms that may be involved in complete } \\
\text { reductive dechlorination } \\
\text { - Identify organisms involved in oxidative } \\
\text { processes (organisms not ID) } \\
\text { - Determine the relative contribution of anaerobic } \\
\text { oxidation in a site's natural attenuation capacity } \\
\text { - Determine the organisms involved in } \\
\text { fermentative processes } \\
\text { - Identify additional unique gene sequences that } \\
\text { can be used to identify key microbial species } \\
\text { involved in the degradation of chlorinated solvent }\end{array}$ & $\begin{array}{l}\text { - What other organisms are } \\
\text { contributing (other than the } \\
\text { classical anaerobic dechlorination } \\
\text { and halorespiration). } \\
\text { - Some anecdotal evidence for other } \\
\text { processes in mass balances at sites } \\
\text { and in mesocosoms. } \\
\text { - Low hanging fruit possible. } \\
\text { - Critical studies and results are } \\
\text { possible in a short time (based on } \\
\text { past research) that would directly } \\
\text { feed into improvements in } \\
\text { attenuation capacity and directly } \\
\text { documenting the presence of an } \\
\text { MNA process. }\end{array}$ & $\begin{array}{l}\text { Work need to be strongly } \\
\text { aligned with attenuation capacity } \\
\text { framework, and to result in } \\
\text { direct characterization tools and } \\
\text { information for modeling. }\end{array}$ & $\mathrm{N}$ to $\mathrm{M}$ & $\begin{array}{l}\$ \text { to } \\
\$ \$\end{array}$ & na & $\begin{array}{l}\mathrm{N} \text { to } \mathrm{M} \\
\text { High priority }\end{array}$ & M & $\mathbf{H}$ \\
\hline $\begin{array}{l}\text { Develop new approaches to long-term monitoring } \\
\text { of Natural Attenuation } \\
\text { - Ecological measurements } \\
\text { - Method to measure Flux (both water and } \\
\text { contaminant) } \\
\text { - New monitoring methods at system } \\
\text { interfaces (e.g. hyporheic zone) } \\
\text { - "Whole earth" sensors } \\
\text { - Failure indicators }\end{array}$ & $\begin{array}{l}\text { - General need to move toward } \\
\text { more direct measures and to make } \\
\text { the long-term stage of monitoring } \\
\text { more streamlined and less } \\
\text { expensive. } \\
\text { - Past results for LTM of caps and } \\
\text { barriers resulted in promising } \\
\text { configurations as listed. } \\
\text { Specific science targets described } \\
\text { below. }\end{array}$ & $\begin{array}{l}\text { - Techniques do not always exist } \\
\text { yet. } \\
\text { - Any progress in this area would } \\
\text { require verification and } \\
\text { comparison with standard } \\
\text { approaches. } \\
\text { - Need to configure development } \\
\text { activities to solving the problem } \\
\text { in terms of MNA/EPR. } \\
\text { - Maintain focus on capacity and } \\
\text { loading balance as the output. } \\
\end{array}$ & & & & & & $\mathbf{H}$ \\
\hline $\begin{array}{l}\text { Scenarios - Taxonomic key to help define } \\
\text { multiple lines of evidence. }\end{array}$ & $\begin{array}{l}\text { - Analogy to biological keys used } \\
\text { to identify plants and animals. } \\
\text { - Key leads to sheet of what } \\
\text { monitoring parameters are most } \\
\text { useful, and why to measure.... } \\
\text { - Currently lacking in protocol and } \\
\text { is not a screening sheet like the } \\
\text { screening sheet in EPA technical } \\
\text { protocol that has been negatively } \\
\text { reviewed by NAS. } \\
\text { - Needs to be created to encourage } \\
\text { people to think. }\end{array}$ & $\begin{array}{l}\text { - May result in a large number of } \\
\text { scenarios and appear complex } \\
\text { and overwhelming. } \\
\text { - May, like some expert systems, } \\
\text { lead to a point where people in } \\
\text { the field are not thinking. }\end{array}$ & $\begin{array}{c}\mathrm{N} \\
\text { • Develop } \\
\text { framework and } \\
\text { put science over } \\
\text { near term period. } \\
\text { - Process helps } \\
\text { focus and } \\
\text { provides value } \\
\text { even during } \\
\text { development. }\end{array}$ & $\$ \$$ & $\mathrm{~N}$ & $\mathbf{N}$ & $\mathrm{M}$ & $\mathbf{H}$ \\
\hline
\end{tabular}




\begin{tabular}{|c|c|c|c|c|c|c|c|c|}
\hline \multirow{2}{*}{ Technical Concept or Objective } & \multirow{2}{*}{ Pros } & \multirow{2}{*}{ Cons } & \multirow[t]{2}{*}{ R\&D Timeframe } & \multirow[t]{2}{*}{ cost } & \multicolumn{3}{|c|}{ Category } & \multirow[t]{2}{*}{ Overall Ranking } \\
\hline & & & & & Policy & Technical & Implementation & \\
\hline $\begin{array}{l}\text { Direct measures and sensors for abiotic } \\
\text { attenuation mechanisms. e.g. resistive fraction; } \\
\text { develop method to measure; results could be used } \\
\text { to est. capacity as well as provide concentration at } \\
\text { which to transition from active treatments }\end{array}$ & $\begin{array}{l}\text { - Sensor to measure attenuation } \\
\text { mechanism directly taking } \\
\text { advantage of rate limited sorption. } \\
\text { - Irreversible sorption and related } \\
\text { factors may dominate MNA/EPR at } \\
\text { many DOE sites such as at SRS. } \\
\text { - There is literature on this topic } \\
\text { that may be directly useful such as } \\
\text { discussed in natural attenuation } \\
\text { process line of inquiry, dual } \\
\text { sorption approach, and research } \\
\text { using supercritical extraction and } \\
\text { accelerated weathering. }\end{array}$ & $\begin{array}{l}\text { Irreversible sorption is not } \\
\text { destruction mechanisms and is } \\
\text { likely to require robust } \\
\text { documentation for acceptance. }\end{array}$ & $\mathrm{N}$ & $\$$ & $\mathrm{~N}$ to $\mathrm{M}$ & $\mathrm{N}$ & $\mathrm{M}$ & H \\
\hline $\begin{array}{l}\text { Development of specific alternative monitoring } \\
\text { configurations to collect data at substantial cost } \\
\text { savings during system performance monitoring } \\
\text { stage } \\
\text { Need near term validation of integrating and flux } \\
\text { type measurements to evaluate the global health } \\
\text { of a MNA system }\end{array}$ & $\begin{array}{l}\text { - Most sites take point } \\
\text { measurements in a few monitoring } \\
\text { wells. } \\
\text { - A flux measurement in a single } \\
\text { horizontal well (or other } \\
\text { configuration) may be a } \\
\text { replacement for several wells - } \\
\text { validation could use a liner and } \\
\text { point sampling for validation. } \\
\text { - Flux meters that use a point } \\
\text { concentration and tracer dilution } \\
\text { are promising. } \\
\text { - Early data on electrical resistance } \\
\text { surveys show potential utility. } \\
\text { - Provides a more direct of flux or } \\
\text { loading. }\end{array}$ & $\begin{array}{l}\text { - Not a traditional monitoring } \\
\text { tool and would require } \\
\text { acceptance. } \\
\text { - Any technology would need to } \\
\text { be validated for each specific } \\
\text { site (probably okay since long } \\
\text { term monitoring assumes that } \\
\text { site is understood) and for a } \\
\text { range of conditions for } \\
\text { widespread use. } \\
\text { - Flux measurement still have to } \\
\text { be interpreted within the context } \\
\text { of transport. }\end{array}$ & \begin{tabular}{|l|}
$\mathrm{N}$ or M \\
- Need to validate \\
and compare to \\
baseline \\
sampling. \\
- Can it be \\
beneficially used?
\end{tabular} & $\begin{array}{l}\$ \text { to } \\
\$ \$\end{array}$ & $\mathrm{M}$ & $\mathbf{N}$ to $\mathrm{M}$ & $\mathbf{N}$ & $\begin{array}{c}\mathbf{H} \\
\text { Need to identify } \\
\text { low hanging fruit to } \\
\text { use as exemplar in } \\
\text { field studies. }\end{array}$ \\
\hline
\end{tabular}




\begin{tabular}{|c|c|c|c|c|c|c|c|c|}
\hline \multirow[t]{2}{*}{ Technical Concept or Objective } & \multirow[t]{2}{*}{ Pros } & \multirow[t]{2}{*}{ Cons } & \multirow[t]{2}{*}{ R\&D Timeframe } & \multirow[t]{2}{*}{ cost } & \multicolumn{3}{|c|}{ Category } & \multirow[t]{2}{*}{ Overall Ranking } \\
\hline & & & & & Policy & Technical & Implementation & \\
\hline $\begin{array}{l}\text { New sentinel monitoring strategies } \\
\text { Threshold and binary sensing }\end{array}$ & $\begin{array}{l}\text { - Potential to provide sentinel } \\
\text { information at a lower cost than a } \\
\text { monitoring well sampling process } \\
\text { or a sensor that requires a wide } \\
\text { dynamic range. } \\
\text { - Many sensors and concepts have } \\
\text { this capability. } \\
\text { - Could be a good way to trigger } \\
\text { more detailed or sensitive sampling } \\
\text { and build in contingencies. } \\
\text { - Potentially inexpensive way to } \\
\text { monitor system parameters of an } \\
\text { extended time frame. }\end{array}$ & $\begin{array}{l}\text { - May need to trigger at a low } \\
\text { value to be an effective sensor. } \\
\text { - Need to operate over a long } \\
\text { time frame to be most useful to } \\
\text { MNA/EPR. } \\
\text { - Difficult to differentiate among } \\
\text { many possible sensors that might } \\
\text { be used. } \\
\text { - Need to differentiate false } \\
\text { negatives and positive (perhaps } \\
\text { run in normally on mode and } \\
\text { check when system turns off). } \\
\text { - Need to have sufficient } \\
\text { specificity to appropriate target } \\
\text { constituents. } \\
\text { - Probably best used in long term } \\
\text { monitoring stage after the } \\
\text { process monitoring has } \\
\text { documented that sensor will be } \\
\text { appropriate for meeting } \\
\text { objectives. }\end{array}$ & $\mathrm{N}$ & $\$$ & $\mathrm{~N}$ & $\mathbf{N}$ & $\mathrm{N}$ & H \\
\hline
\end{tabular}




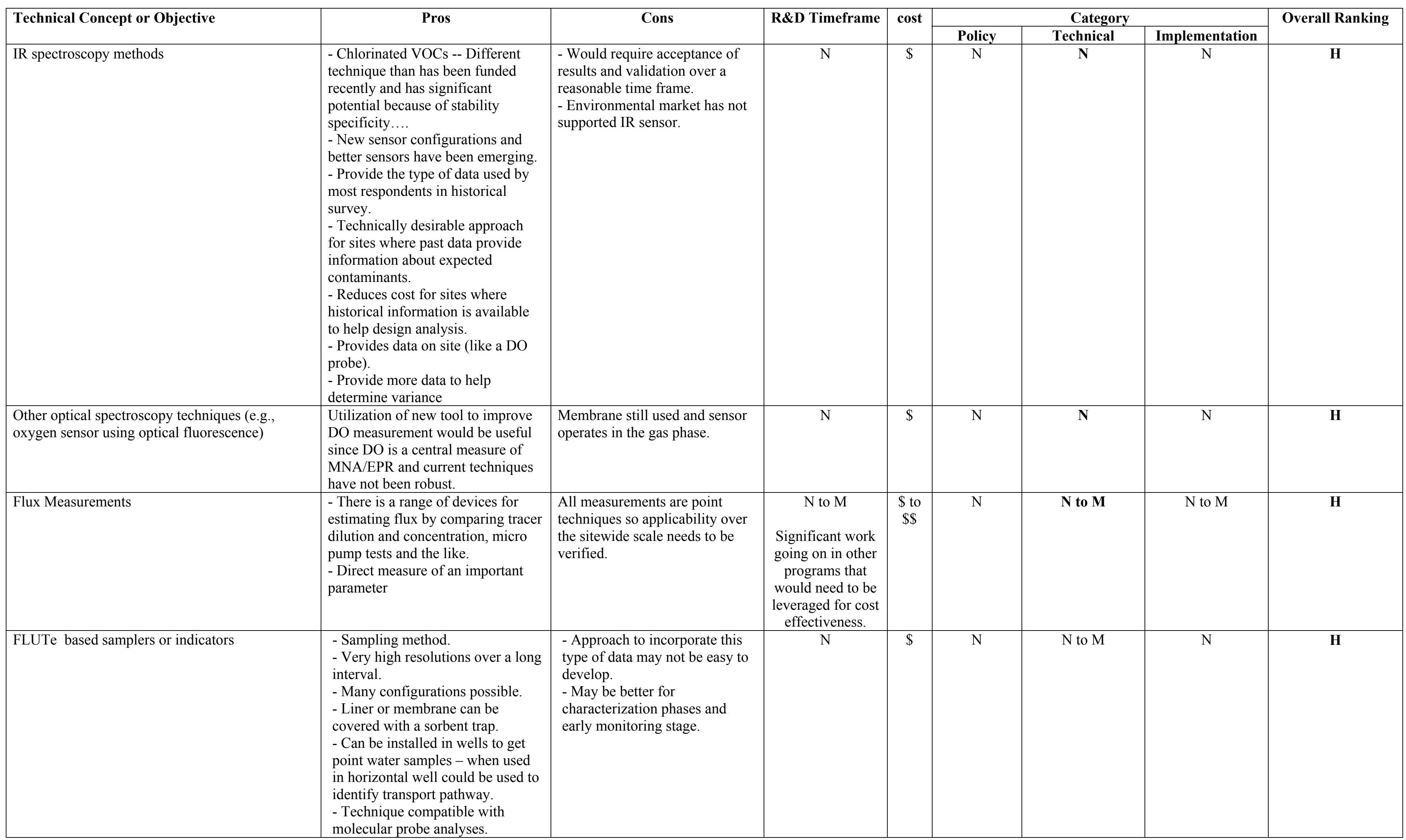




\begin{tabular}{|c|c|c|c|c|c|c|c|c|}
\hline \multirow[t]{2}{*}{ Technical Concept or Objective } & \multirow[t]{2}{*}{ Pros } & \multirow[t]{2}{*}{ Cons } & \multirow[t]{2}{*}{ R\&D Timeframe } & \multirow[t]{2}{*}{ cost } & \multicolumn{3}{|c|}{ Category } & \multirow[t]{2}{*}{ Overall Ranking } \\
\hline & & & & & Policy & Technical & Implementation & \\
\hline $\begin{array}{l}\text { Monitoring of integrating or bioconcentrating } \\
\text { indicator species such as honeybees, mollusks, or } \\
\text { fish - for systems where information on low } \\
\text { concentrations and patchy releases would be } \\
\text { useful. }\end{array}$ & $\begin{array}{l}\text { - Provide a sensitive technique to } \\
\text { monitor low level exposure and } \\
\text { possibly an integration of } \\
\text { heterogeneous and patchy } \\
\text { discharges over large areas. } \\
\text { - There is a large body of literature } \\
\text { in this area that could be further } \\
\text { examined and leveraged as needed. }\end{array}$ & $\begin{array}{l}\text { - Requires contaminant to impact } \\
\text { ecosystem before measurement } \\
\text { is diagnostic - may not } \\
\text { appropriately address MNA/EPR } \\
\text { objectives. } \\
\text { - Not a direct measure of MNA } \\
\text { processes and, thus may not } \\
\text { move monitoring in the desired } \\
\text { direction for "more direct } \\
\text { measures" of processes. } \\
\text { - Would require clear } \\
\text { documentation that measurement } \\
\text { is diagnostic that progress } \\
\text { toward remediation objectives is } \\
\text { tied to indicator. }\end{array}$ & $M$ to $L$ & $\$ \$$ & $\mathrm{M}$ to $\mathrm{L}$ & $\mathrm{M}$ to $\mathrm{L}$ & $\mathrm{M}$ to $\mathrm{L}$ & M \\
\hline $\begin{array}{l}\text { Monitoring of general ecosystem structure and } \\
\text { function (using diversity indices, trophic level } \\
\text { indices, and the like)- to efficiently document } \\
\text { minimal ecological impacts. }\end{array}$ & $\begin{array}{l}\text { - Promising for documenting the } \\
\text { general ecological stability and } \\
\text { robustness in the discharge zone } \\
\text { over a long period of time. } \\
\text { - A large body of research has been } \\
\text { generated on this topic that might } \\
\text { provide useful specific indices that } \\
\text { are both sensitive and yet only } \\
\text { alarm if truly disruptive ecological } \\
\text { impacts are being expressed. } \\
\text { - Corps of Engineers uses this } \\
\text { approach for their dredging } \\
\text { program as a real world example. }\end{array}$ & $\begin{array}{l}\text { - Requires contaminant to impact } \\
\text { ecosystem before measurement } \\
\text { is diagnostic - may not } \\
\text { appropriately address MNA/EPR } \\
\text { objectives. } \\
\text { - Not a direct measure of MNA } \\
\text { processes and, thus may not } \\
\text { move monitoring in the desired } \\
\text { direction for "more direct } \\
\text { measures" of processes (may be } \\
\text { a measure that could be used or } \\
\text { set as a remediation goal } \\
\text { however) }\end{array}$ & $\mathrm{M}$ to $\mathrm{L}$ & $\$ \$$ & M to $\mathrm{L}$ & M to $\mathrm{L}$ & $\mathrm{M}$ to $\mathrm{L}$ & $\mathrm{M}$ \\
\hline $\begin{array}{l}\text { Geophysical techniques - particularly electrical } \\
\text { methods using a permanent electrode array -- for } \\
\text { cases where changes in conductivity are related to } \\
\text { loading or the maintenance of appropriate } \\
\text { chemical conditions for attenuation processes to } \\
\text { occur. }\end{array}$ & $\begin{array}{l}\text { - Measures subsurface properties } \\
\text { over a volume and provides data } \\
\text { that is somewhat more robust to } \\
\text { heterogeneity. } \\
\text { - When used to monitor differences } \\
\text { or changes, as would be done in } \\
\text { monitoring, these tools become } \\
\text { more powerful. }\end{array}$ & $\begin{array}{l}\text { - Some types difficult to } \\
\text { implement for long term } \\
\text { monitoring because of stability } \\
\text { of electrodes. } \\
\text { - Might be more expensive than } \\
\text { baseline in some cases. } \\
\text { - Not a direct measure of MNA } \\
\text { processes and, thus may not } \\
\text { move monitoring in the desired } \\
\text { direction for "more direct } \\
\text { measures" of processes. } \\
\text { - Would require clear } \\
\text { documentation that measurement } \\
\text { is diagnostic that progress } \\
\text { toward remediation objectives is } \\
\text { tied to indicator. } \\
\end{array}$ & M & $\$ \$$ & $\mathrm{M}$ to $\mathrm{L}$ & $\mathrm{M}$ to $\mathrm{L}$ & $\mathrm{M}$ to $\mathrm{L}$ & M \\
\hline
\end{tabular}




\begin{tabular}{|c|c|c|c|c|c|c|c|c|}
\hline \multirow[t]{2}{*}{ Technical Concept or Objective } & \multirow[t]{2}{*}{ Pros } & \multirow[t]{2}{*}{ Cons } & \multirow[t]{2}{*}{ R\&D Timeframe } & \multirow[t]{2}{*}{ cost } & \multicolumn{3}{|c|}{ Category } & \multirow[t]{2}{*}{ Overall Ranking } \\
\hline & & & & & Policy & Technical & Implementation & \\
\hline $\begin{array}{l}\text { Multispectral and hyperspectral imagery } \\
\text { correlated to contaminant exposure and impacts. }\end{array}$ & $\begin{array}{l}\text { - Similar in advantages to } \\
\text { ecosystem indices but with the } \\
\text { major advantage that monitoring is } \\
\text { done remotely and costs could be } \\
\text { low based on commercially } \\
\text { available data. } \\
\text { - Multispectral and hyperspectral } \\
\text { studies have already shown spectral } \\
\text { signatures associated with plume } \\
\text { outcrops (primarily stress) and } \\
\text { more specific changes with } \\
\text { individual metals. }\end{array}$ & $\begin{array}{l}\text { - Signal may not be specific and } \\
\text { would require a large amount of } \\
\text { basic research to achieve } \\
\text { success. } \\
\text { - Requires contaminant to impact } \\
\text { ecosystem before measurement } \\
\text { is diagnostic - may not } \\
\text { appropriately address MNA/EPR } \\
\text { objectives. } \\
\text { - Not a direct measure of MNA } \\
\text { processes and, thus may not } \\
\text { move monitoring in the desired } \\
\text { direction for "more direct } \\
\text { measures" of processes (may be } \\
\text { a measure that could be used or } \\
\text { set as a remediation goal } \\
\text { however) }\end{array}$ & $\mathrm{L}$ & $\$ \$ \$$ & $\mathrm{~L}$ & $\mathrm{~L}$ & $\mathrm{~L}$ & $\mathrm{H}$ \\
\hline $\begin{array}{l}\text { Develop methods to measure sustainability of } \\
\text { process }\end{array}$ & $\begin{array}{l}\text { - General question about how to } \\
\text { measure sustainability over a short } \\
\text { period of time and have confidence } \\
\text { that the process would project over } \\
\text { the necessary period of MNA/EPR. } \\
\text { - Limited direct measures at this } \\
\text { time. } \\
\text { - Current SERDP work. }\end{array}$ & $\begin{array}{l}\text { - Critical issue that will require } \\
\text { linking to related research } \\
\text { organizations. } \\
\text { - Large topic that is unlikely to } \\
\text { be resolved in a limited time and } \\
\text { with limited resources. }\end{array}$ & $\mathrm{L}$ & $\begin{array}{l}\$ \$ \text { to } \\
\$ \$ \$\end{array}$ & $\mathrm{~L}$ & $\mathrm{~L}$ & $\mathrm{~L}$ & M \\
\hline $\begin{array}{l}\text { Look at methods to directly measure risk and } \\
\text { potentially system capacity. e.g. DNA on a chip }\end{array}$ & $\begin{array}{l}\text { Risk sensor would be a direct } \\
\text { measure of potential regulatory } \\
\text { target or goal. }\end{array}$ & $\begin{array}{l}\text { - Past toxicity measuring } \\
\text { systems have shown variable } \\
\text { success. } \\
\text { - Any progress would require } \\
\text { validation and verification and } \\
\text { be applicable to long term } \\
\text { performance monitoring phase. }\end{array}$ & $\mathrm{M}$ to $\mathrm{L}$ & $\begin{array}{l}\$ \$ \text { to } \\
\$ \$ \$\end{array}$ & $\mathrm{~L}$ & $\mathrm{~L}$ & $\mathrm{~L}$ & M \\
\hline
\end{tabular}




\begin{tabular}{|c|c|c|c|c|c|c|c|c|}
\hline \multirow[t]{2}{*}{ Technical Concept or Objective } & \multirow[t]{2}{*}{ Pros } & \multirow[t]{2}{*}{ Cons } & \multirow[t]{2}{*}{ R\&D Timeframe } & \multirow[t]{2}{*}{ cost } & \multicolumn{3}{|c|}{ Category } & \multirow[t]{2}{*}{ Overall Ranking } \\
\hline & & & & & Policy & Technical & Implementation & \\
\hline Heterogeneous Flow Measurements & $\begin{array}{l}\text { - Measure preferential flow paths } \\
\text { over the volume of interest. } \\
\text { - Small scale variants include } \\
\text { borehole flowmeter and buried } \\
\text { flowmeters. } \\
\text { - Small scale pump tests and } \\
\text { injection extraction tests also } \\
\text { possible. } \\
\text { - Possible use of geophysics. }\end{array}$ & $\begin{array}{l}\text { Limited tools and techniques } \\
\text { available. } \\
\text { Available tools tend to provide } \\
\text { data that are 2D rather than 3D. }\end{array}$ & $\mathrm{M}$ to $\mathrm{L}$ & $\begin{array}{l}\$ \text { to } \\
\$ \$\end{array}$ & M & $\mathrm{M}$ to $\mathrm{L}$ & M & M \\
\hline $\begin{array}{l}\text { Fiber optic sensors (e.g., evanescent } \\
\text { wave/cladding sensors) }\end{array}$ & $\begin{array}{l}\text { Long sensing pathlength that might } \\
\text { provide an integrated value over the } \\
\text { deployment zone. }\end{array}$ & $\begin{array}{l}\text { Specificity, installation, } \\
\text { sensitivity, durability, and } \\
\text { verification versus accepted } \\
\text { methods. }\end{array}$ & $\mathrm{M}$ to $\mathrm{L}$ & $\begin{array}{l}\$ \$ \text { to } \\
\$ \$ \$\end{array}$ & $\mathrm{~L}$ & $\mathrm{~L}$ & $\mathrm{~L}$ & M \\
\hline $\begin{array}{l}\text { Inexpensive rapid access techniques coupled with } \\
\text { sensors or inexpensive samplers }\end{array}$ & $\begin{array}{l}\text { - Replace fixed wells with periodic } \\
\text { probe sampling. } \\
\text { - Advantage might be access to } \\
\text { soils that are a more direct indicator } \\
\text { of microbial processes. }\end{array}$ & $\begin{array}{l}\text { - May be more expensive. } \\
\text { - Reduces statistical bases for } \\
\text { comparison and does not provide } \\
\text { access for sampling over time. }\end{array}$ & $\mathrm{N}$ & $\$$ & $\mathrm{M}$ & $\mathrm{N}$ & $\mathrm{N}$ & M \\
\hline $\begin{array}{l}\text { Controlling/Master Variable Sensors (e.g., } \\
\text { Pressure, Temp, pH, Eh, } \mathrm{O}_{2} \text {, flow, moisture) }\end{array}$ & $\begin{array}{l}\text { Standard sensors that may not be in } \\
\text { wide use. }\end{array}$ & $\begin{array}{l}\text { Specificity, installation, } \\
\text { sensitivity, durability, and } \\
\text { verification versus accepted } \\
\text { methods. }\end{array}$ & $\mathrm{N}$ & $\$$ & na & $\mathrm{N}$ to $\mathrm{M}$ & $\mathrm{N}$ to $\mathrm{M}$ & M \\
\hline Open Path FTIR and other non point monitoring & $\begin{array}{l}\text { Provides integrated measure that is } \\
\text { more closely tied to flux. }\end{array}$ & $\begin{array}{l}\text { - Sensitivity and verification } \\
\text { versus other methods. } \\
\text { - Fluxes vary widely as a } \\
\text { function of time and conditions } \\
\text { and how data incorporated into } \\
\text { MNA would need to be } \\
\text { developed. }\end{array}$ & $\mathrm{M}$ to $\mathrm{L}$ & $\begin{array}{l}\$ \text { to } \\
\$ \$\end{array}$ & M & $\mathrm{M}$ to $\mathrm{L}$ & $\mathrm{M}$ to $\mathrm{L}$ & M \\
\hline
\end{tabular}




\section{Most Promising Process and Enhancement Science and Technology Targets}

KEY:

R\&D Timeframe: $\mathrm{N}$ (near-term) $<2$ years, $\mathrm{M}$ (medium-term) $2-5$ years, $\mathrm{L}$ (long-term) $>5$ years. cost $\$=\leq \$ 100 \mathrm{~K}, \$ \$=>\$ 100 \mathrm{~K}-\$ 500 \mathrm{~K}, \$ \$ \$=>\$ 500 \mathrm{~K}$

Overall Ranking: High (H) , Medium (M) or Low (L)

\begin{tabular}{|c|c|c|c|c|c|c|c|c|}
\hline \multirow[t]{2}{*}{ Technical Concept or Objective } & \multirow[t]{2}{*}{ Pros } & \multirow[t]{2}{*}{ Cons } & \multirow[t]{2}{*}{ R\&D Timeframe } & \multirow[t]{2}{*}{ cost } & \multicolumn{3}{|c|}{ Category } & \multirow[t]{2}{*}{ Overall Ranking } \\
\hline & & & & & Policy & Technical & Implementation & \\
\hline $\begin{array}{l}\text { Microbiology - Bioaugmentation } \\
\text { - Anaerobic systems (with Dehalococcoides) } \\
\text { - Aerobic systems (with JS666) } \\
\\
\text { Valuable to determine answers to ecological } \\
\text { questions - why are dechlorinators present in } \\
\text { some systems but not others? This would } \\
\text { provide both fundamental and practical } \\
\text { results. } \\
\text { (Natural Processes - Enhancements) }\end{array}$ & $\begin{array}{l}\text { - Add key organisms to } \\
\text { facilitate complete removal or } \\
\text { to enhance capacity. } \\
\text { - Most applicable in MNA/PR } \\
\text { sense if conditions are present } \\
\text { but organisms missing.... } \\
\text { - Recent improvements in } \\
\text { delivery (emulsification, etc) } \\
\text { are applicable. }\end{array}$ & $\begin{array}{l}\text { - Confirmation of linkage of } \\
\text { need to add organisms to } \\
\text { overcome cis-DCE stall needed. } \\
\text { - Microbial transport and } \\
\text { distribution issues may limit } \\
\text { implementability. } \\
\text { - If electron donor needed, then } \\
\text { same issues as above. }\end{array}$ & $\begin{array}{l}\mathrm{N} \text { to } \mathrm{M} \\
\text { - Active emerging } \\
\text { research area. } \\
\text { - Need answers to } \\
\text { specific key } \\
\text { questions identified } \\
\text { in line of inquiry. }\end{array}$ & $\$ \$$ & $\mathrm{M}$ & $\begin{array}{c}\mathrm{N} \text { to } \mathrm{M} \\
\text { High priority }\end{array}$ & $\mathrm{M}$ & $\begin{array}{l}\text { H } \\
\text { High rated } \\
\text { enhancement idea } \\
\text { that links to high } \\
\text { rated bioassessment } \\
\text { tools. }\end{array}$ \\
\hline $\begin{array}{l}\text { Modifying Large-scale Hydrology - } \\
\text { - Bypass of upgradient water } \\
\text { - Decrease infiltration throughout plume } \\
\text { increase runoff } \\
\text { increase evapotranspiration } \\
\text { - Decrease mass discharge from source } \\
\text { - Bypass of competing electron acceptors }\end{array}$ & $\begin{array}{l}\text { - Conceptually simple. } \\
\text { - Standard engineering practice. } \\
\text { - Easy to monitor. } \\
\text { - Simple to include in models. } \\
\text { - Large body of containment } \\
\text { literature. } \\
\text { - Main value of line of inquiry } \\
\text { is to show how hydrology } \\
\text { integrates with MNA/EPR - by } \\
\text { setting loading goals and } \\
\text { bypass. }\end{array}$ & $\begin{array}{l}\text { Barriers often looked down upon } \\
\text { because they do not directly } \\
\text { destroy mass (the changes they } \\
\text { cause however may increase the } \\
\text { efficiency of mass destruction as } \\
\text { noted in pros) }\end{array}$ & $\mathrm{N}$ to $\mathrm{M}$ & $\$$ to $\$ \$$ & $\mathrm{Na}$ & $\mathrm{M}$ & $\mathbf{N}$ to $\mathrm{M}$ & $\begin{array}{c}\mathrm{H} \\
\text { Selected primarily } \\
\text { as an } \\
\text { implementation } \\
\text { option if } \\
\text { appropriate for use } \\
\text { with other project } \\
\text { activities }\end{array}$ \\
\hline
\end{tabular}




\begin{tabular}{|c|c|c|c|c|c|c|c|c|}
\hline \multirow[t]{2}{*}{ Technical Concept or Objective } & \multirow[t]{2}{*}{ Pros } & \multirow[t]{2}{*}{ Cons } & \multirow[t]{2}{*}{ R\&D Timeframe } & \multirow[t]{2}{*}{ cost } & \multicolumn{3}{|c|}{ Category } & \multirow[t]{2}{*}{ Overall Ranking } \\
\hline & & & & & Policy & Technical & Implementation & \\
\hline $\begin{array}{l}\text { Phytoremediation - Natural Vegetation with No } \\
\text { Enhancements } \\
\text { - Determine the spatial variability in rhizosphere } \\
\text { responses to contaminant degradation and uptake } \\
\text { - Identify mechanisms that enhance chlorinated } \\
\text { ethene biodegradation in the rhizosphere } \\
\text { - Evaluate the interaction among plants, soil } \\
\text { microorganisms, and contamination especially in } \\
\text { the rhizosphere } \\
\text { - Evaluate the interaction among plants, soil } \\
\text { microorganisms, and contamination especially in } \\
\text { the rhizosphere } \\
\text { - Assess seasonal and climatic influences on } \\
\text { phytoremediation (plants species, rates) }\end{array}$ & $\begin{array}{l}\text { Understanding degradation } \\
\text { rates and processes in natural } \\
\text { plant ecosystems. }\end{array}$ & $\begin{array}{l}\text { Occurs near outcrop and requires } \\
\text { plume to expand to treatment } \\
\text { zone. }\end{array}$ & $\begin{array}{c}\text { N to M } \\
\text { • Significant } \\
\text { ongoing work - any } \\
\text { use in this project } \\
\text { would need to } \\
\text { strongly leverage } \\
\text { that effort. } \\
\text { - Difficult to } \\
\text { understand seasonal } \\
\text { and climatic } \\
\text { influences in } \\
\text { limited study } \\
\text { period. }\end{array}$ & $\$$ to $\$ \$$ & $\mathrm{M}$ & $\mathbf{M}$ & $\mathrm{Na}$ & M \\
\hline Phytoremediation - Fertilization strategy & $\begin{array}{l}\text { - Boosting biomass using } \\
\text { simple manipulations. } \\
\text { - Significant amount of work } \\
\text { going on for natural phyto and } \\
\text { compounds (e.g., } \\
\text { phytoalkaloids) contribute to } \\
\text { degradation of specific targets. }\end{array}$ & $\begin{array}{l}\text { - Occurs near outcrop and } \\
\text { requires plume to expand to } \\
\text { treatment zone. } \\
\text { - Requires long period of } \\
\text { research for effective proof } \\
\text { because of growing seasons and } \\
\text { variability. } \\
\text { - Is this sustainable? } \\
\text { - Can this be implemented } \\
\text { within the MNA/EPR context? } \\
\text { - May fall near the boundary } \\
\text { between passive and active } \\
\text { remediation and not fit within } \\
\text { project bounds. }\end{array}$ & M & $\$ \$$ & M & $\mathbf{M}$ & M & M \\
\hline Phytoremediation - Layered construction & $\begin{array}{l}\text { - Sequencing plants for better } \\
\text { effect - shallow and deep roots, } \\
\text { plants that grow throughout } \\
\text { season. } \\
\text { - Involves interventions }\end{array}$ & $\begin{array}{l}\text { - Occurs near outcrop and } \\
\text { requires plume to expand to } \\
\text { treatment zone. } \\
\text { - Even longer term than above. } \\
\text { - Can this be implemented } \\
\text { within the MNA/EPR context? } \\
\text { - May fall near the boundary } \\
\text { between passive and active } \\
\text { remediation and not fit within } \\
\text { project bounds. }\end{array}$ & $\mathrm{L}$ & $\$ \$$ & $\mathrm{M}$ & $\mathbf{M}$ & $\mathrm{M}$ & $\mathrm{M}$ \\
\hline
\end{tabular}




\begin{tabular}{|c|c|c|c|c|c|c|c|c|}
\hline \multirow[t]{2}{*}{ Technical Concept or Objective } & \multirow[t]{2}{*}{ Pros } & \multirow[t]{2}{*}{ Cons } & \multirow[t]{2}{*}{ R\&D Timeframe } & \multirow[t]{2}{*}{ cost } & \multicolumn{3}{|c|}{ Category } & \multirow[t]{2}{*}{ Overall Ranking } \\
\hline & & & & & Policy & Technical & Implementation & \\
\hline $\begin{array}{l}\text { Phytoremediation - Genetic transformations } \\
\text { Species/clone selection: } \\
\text { - Widely adapted } \\
\text { - Carbon to root production } \\
\text { - Amount and type of exudates } \\
\text { - } \quad \text { Function under stress } \\
\text { - Metabolism of contaminants } \\
\text { - Water uptake }\end{array}$ & $\begin{array}{l}\text { Customized species selection or } \\
\text { assemblage. Current example, } \\
\text { Hybrid Poplar, sequenced soon } \\
\text { and can be manipulated. }\end{array}$ & $\begin{array}{l}\text { - Occurs near outcrop and } \\
\text { requires plume to expand to } \\
\text { treatment zone. } \\
\text { - Even longer term than above. } \\
\text { - Can this be implemented } \\
\text { within the MNA/EPR context? } \\
\text { - May fall near the boundary } \\
\text { between passive and active } \\
\text { remediation and not fit within } \\
\text { project bounds. }\end{array}$ & $\mathrm{L}$ & $\$ \$$ & $\mathrm{M}$ & $\mathbf{M}$ & $\mathrm{M}$ & M \\
\hline Consider volatilization from surface water. & $\begin{array}{l}\text { - Technically feasible and could } \\
\text { be a significant component in } \\
\text { attenuation capacity. } \\
\text { - May be acceptable if } \\
\text { considered in terms of load like } \\
\text { a NPDES or air discharge. } \\
\text { - Particularly well suited to } \\
\text { VOCs because of their gas } \\
\text { partitioning. } \\
\text { - Would be quite useful for } \\
\text { chlorinated VOCs that often } \\
\text { have some tendency to outcrop. }\end{array}$ & $\begin{array}{l}\text { - Requires acceptance of } \\
\text { discharge into surface water. } \\
\text { - Requires acceptance of release } \\
\text { to the atmosphere. } \\
\text { - Requires no impact on local } \\
\text { ecological receptors or sensitive } \\
\text { species - requires careful setting } \\
\text { of goals. }\end{array}$ & $\begin{array}{l}\text { N } \\
\text { • Requires } \\
\text { monitoring of a } \\
\text { zone that is not } \\
\text { normally } \\
\text { monitored. } \\
\text { •Linked to } \\
\text { modeling as well. }\end{array}$ & $\$$ & $\mathbf{N}$ & $\mathrm{N}$ & $\mathrm{M}$ & $\mathrm{M}$ \\
\hline Expand groundwater-surface water zone. & $\begin{array}{l}\text { - Increasing the attenuation } \\
\text { capacity in a zone where the } \\
\text { capacity is already recognized } \\
\text { and where natural conditions } \\
\text { may help maintain } \\
\text { sustainability. } \\
\text { - Engineered wetlands } \\
\text { increasingly accepted. } \\
\text { - A lot of work nationally on } \\
\text { elated topics. }\end{array}$ & $\begin{array}{l}\text { - Occurs at receptor boundary } \\
\text { and requires plume to expand to } \\
\text { that location. } \\
\text { - For large engineering versions, } \\
\text { occurs in a sensitive ecological } \\
\text { area. } \\
\text { - Realizing full potential, then } \\
\text { VOCs have to penetrate into } \\
\text { reaction zone. } \\
\text { - May fall near the boundary } \\
\text { between passive and active } \\
\text { remediation and not fit within } \\
\text { project bounds. }\end{array}$ & $\begin{array}{l}\text { M } \\
\text { - Near term efforts } \\
\text { would need to } \\
\text { examine low } \\
\text { impact variants for } \\
\text { increasing capacity. } \\
\text { - Demonstration } \\
\text { will require longer } \\
\text { term effort. }\end{array}$ & $\$$ to $\$ \$$ & $\mathrm{M}$ & $\mathbf{M}$ & $\mathrm{M}$ & M \\
\hline Enhance FeS precipitation (biostimulation) & $\begin{array}{l}\text { - With enough FeS, process } \\
\text { could last a long time. } \\
\text { - Best applied to anaerobic } \\
\text { plume for sustainable use. } \\
\text { - Primarily engineering and } \\
\text { most feasible for small } \\
\text { sites/plumes. } \\
\text { - Some work has been done on } \\
\text { this. }\end{array}$ & $\begin{array}{l}\text { - Sustainable system would } \\
\text { require long term source of } \\
\text { organics and reducing conditions } \\
\text { and possibly long term sulfate } \\
\text { source (e.g., gypsum). } \\
\text { - Difficult to separate biotic and } \\
\text { abiotic effects in practice and } \\
\text { may be hard to directly monitor. } \\
\text { - Works under the same } \\
\text { conditions and anaerobic bio and } \\
\text { so does not expand conditions. }\end{array}$ & $\begin{array}{c}\mathrm{N} \\
\text { Should be able to } \\
\text { piggyback on } \\
\text { related research } \\
\text { programs }\end{array}$ & $\$$ to $\$ \$$ & $\mathrm{Na}$ & $\mathrm{M}$ & $\mathrm{M}$ & $\mathrm{L}$ \\
\hline
\end{tabular}




\begin{tabular}{|c|c|c|c|c|c|c|c|c|}
\hline \multirow{2}{*}{ Technical Concept or Objective } & \multirow{2}{*}{ Pros } & \multirow{2}{*}{ Cons } & \multirow[t]{2}{*}{ R\&D Timeframe } & \multirow[t]{2}{*}{ cost } & \multicolumn{3}{|c|}{ Category } & \multirow[t]{2}{*}{ Overall Ranking } \\
\hline & & & & & Policy & Technical & Implementation & \\
\hline $\begin{array}{l}\text { Create new interfaces. } \\
\text { - permeable reactive barriers }\end{array}$ & $\begin{array}{l}\text { - Robust recent development of } \\
\text { PRBs in general. } \\
\text { - Many variants are possible. }\end{array}$ & $\begin{array}{l}\text { - Limited data on sustainable } \\
\text { barrier development - lots of } \\
\text { work may be needed. } \\
\text { - PRB community has } \\
\text { recognized that hydrology } \\
\text { problems rather than chemistry } \\
\text { can cause problems over time } \\
\text { (flow diversion etc.) } \\
\text { - PRB looks more active. }\end{array}$ & $\begin{array}{c}\text { M } \\
\text { Identify possible } \\
\text { materials to be } \\
\text { evaluated to create } \\
\text { a sustainable } \\
\text { system. }\end{array}$ & $\$ \$$ & $\mathrm{M}$ & $\mathbf{M}$ & M & $\mathrm{L}$ \\
\hline Create vadose-groundwater reaction zone. & $\begin{array}{l}\text { - Would be applicable to } \\
\text { specific sites. } \\
\text { - A few variants possible. }\end{array}$ & $\begin{array}{l}\text { If DNAPL below water table } \\
\text { then not viable. }\end{array}$ & $\begin{array}{c}\text { M } \\
\text { Identify possible } \\
\text { materials to be } \\
\text { evaluated to create } \\
\text { a sustainable } \\
\text { system. }\end{array}$ & $\$ \$$ & M & $\mathbf{M}$ & M & $\mathrm{L}$ \\
\hline $\begin{array}{l}\text { Biostimulation } \\
\text { - Improvements to electron donors } \\
\text { - Passive Delivery }\end{array}$ & $\begin{array}{l}\text { - Looking at longer lasting } \\
\text { electron donors - what is } \\
\text { possible. } \\
\text { - Considerations were } \\
\text { partitioning donors and slow } \\
\text { degradation compounds, peat, } \\
\text { etc. Years, Decades, etc. } \\
\text { - May be an improved } \\
\text { engineering concept as well for } \\
\text { improving delivery over } \\
\text { extended times. } \\
\text { - Recent improvements in } \\
\text { delivery (emulsification, etc) } \\
\text { are applicable. }\end{array}$ & $\begin{array}{l}\text { - Sustainability? } \\
\text { - Can this be implemented } \\
\text { within the MNA/EPR context? } \\
\text { - May fall near the boundary } \\
\text { between passive and active } \\
\text { remediation and not fit within } \\
\text { project bounds. }\end{array}$ & $\begin{array}{c}\text { M } \\
\text { Much research } \\
\text { ongoing in this area } \\
\text { and most } \\
\text { appropriate when } \\
\text { viewed as active } \\
\text { remediation }\end{array}$ & $\$ \$$ & na & $\mathrm{N}$ to $\mathrm{M}$ & $\mathrm{N}$ & $\mathrm{L}$ \\
\hline
\end{tabular}




\section{Most Promising Policy Targets}

KEY

RD Timeframe: $\mathrm{N}$ (near-term) $<2$ years, $\mathrm{M}$ (medium-term) $2-5$ years, $\mathrm{L}$ (long-term) $>5$ years

$\$ \$ \$: \$=\leq \$ 100 \mathrm{~K}, \$ \$=>\$ 100 \mathrm{~K}-\$ 500 \mathrm{~K}, \$ \$ \$=>\$ 500 \mathrm{~K}$

Overall Ranking: High (H) , Medium (M) or Low (L)

\begin{tabular}{|c|c|c|c|c|c|c|c|c|}
\hline \multirow[t]{2}{*}{ Policy Concept or Objective } & \multirow[t]{2}{*}{ Pros } & \multirow[t]{2}{*}{ Cons } & \multirow[t]{2}{*}{ R\&D Timeframe } & \multirow[t]{2}{*}{ cost } & \multicolumn{3}{|c|}{ Category } & \multirow[t]{2}{*}{ Overall Ranking } \\
\hline & & & & & Policy & Technical & Implementation & \\
\hline $\begin{array}{l}\text { Develop decision process (based on science) to } \\
\text { transition from active to more passive } \\
\text { treatment }\end{array}$ & $\begin{array}{l}\text { - Stages support technically } \\
\text { based transition through the } \\
\text { entire characterization and site } \\
\text { remediation effort } \\
\text { - Defines the technical basis for } \\
\text { transition through the science } \\
\text { framework of attenuation } \\
\text { capacity and loading to } \\
\text { facilitate transition from one } \\
\text { stage of site remediation to the } \\
\text { next. }\end{array}$ & $\begin{array}{l}\text { - May not fit with current } \\
\text { regulatory process (e.g., the } \\
\text { RI/FS and ROD scheduling). } \\
\text { - MNA/EPR may need its own } \\
\text { customized track due to the } \\
\text { times needed for verification. }\end{array}$ & na & na & $\mathbf{N}$ & $\mathbf{N}$ & $\mathrm{M}$ & $\mathbf{H}$ \\
\hline $\begin{array}{l}\text { Need concurrence with stages of } \\
\text { characterization and monitoring and alignment } \\
\text { with CERCLA/RCRA etc, decision-making } \\
\text { process. }\end{array}$ & See above & See above & na & na & $\mathbf{N}$ & na & $\mathrm{M}$ to $\mathrm{L}$ & $\mathrm{H}$ \\
\hline $\begin{array}{l}\text { Acceptance of central concept }- \text { Balancing } \\
\text { Attenuation Capacity and Loading }\end{array}$ & $\begin{array}{l}\text { - Easy to understand because it } \\
\text { is consistent with engineering } \\
\text { and other disciplines. } \\
\text { - What is needed is to capture in } \\
\text { the language of the policy for } \\
\text { implementation. } \\
\text { - Consistent with flux } \\
\text { approaches that EPA is } \\
\text { currently emphasizing - } \\
\text { particular value in linking with } \\
\text { mass discharge concepts being } \\
\text { developed for DNAPL sources } \\
\end{array}$ & $\begin{array}{l}\text { - There are uncertainties } \\
\text { associated with loading and } \\
\text { capacity and definitions would } \\
\text { need to be clear to avoid grossly } \\
\text { increasing the amount of data } \\
\text { required. } \\
\text { - Requires guidance, education } \\
\text { and reorienting conceptual base } \\
\text { in an ongoing program. }\end{array}$ & na & na & $\mathbf{N}$ to $\mathrm{M}$ & na & $\mathrm{M}$ to $\mathrm{L}$ & $\mathrm{H}$ \\
\hline $\begin{array}{l}\text { Acceptance of framework for selecting the } \\
\text { analytes and interpreting the results. A } \\
\text { decision tree or scenario based structure would be } \\
\text { examples }\end{array}$ & $\begin{array}{l}\text { - Would provide the user the } \\
\text { answer to "why" certain things } \\
\text { need to be measured at one site } \\
\text { and not at another. } \\
\text { - This is an important policy } \\
\text { issue that is based in the } \\
\text { science. } \\
\text { - Highlights the need to } \\
\text { measure things directly }\end{array}$ & $\begin{array}{l}\text { Will come out naturally in any } \\
\text { updated guidelines for } \\
\text { MNA/EPR protocol } \\
\text { implementation }\end{array}$ & na & na & $\mathbf{N}$ & $\mathrm{N}$ & $\mathrm{M}$ & $\mathrm{H}$ \\
\hline
\end{tabular}




\begin{tabular}{|c|c|c|c|c|c|c|c|c|}
\hline \multirow{2}{*}{ Policy Concept or Objective } & \multirow{2}{*}{ Pros } & \multirow{2}{*}{ Cons } & \multirow{2}{*}{ R\&D Timeframe } & \multirow{2}{*}{ cost } & \multicolumn{3}{|c|}{ Category } & \multirow{2}{*}{ Overall Ranking } \\
\hline & & & & & Policy & Technical & Implementation & \\
\hline $\begin{array}{l}\text { Acceptability of non-degrading processes as part } \\
\text { of natural attenuation }\end{array}$ & $\begin{array}{l}\text { - All processes that move } \\
\text { toward the remediation goal } \\
\text { should be considered. } \\
\text { - As protocols move forward, } \\
\text { the broader processes more } \\
\text { acceptable if framed in terms of } \\
\text { capacity and target goals. }\end{array}$ & $\begin{array}{l}\text { - May not be high priority } \\
\text { because OSWER directive } \\
\text { already recognizes all of the } \\
\text { mechanisms. } \\
\text { - Good science in modeling and } \\
\text { other areas will support progress } \\
\text { in this area without specific } \\
\text { investment. }\end{array}$ & na & na & $\mathbf{N}$ & na & $\mathrm{Na}$ & $\mathrm{L}$ \\
\hline
\end{tabular}

